\title{
SYSTEM IDENTIFICATION AND THE MODELING OF SAILING YACHTS
}

By

\author{
Katrina Legursky
}

Submitted to the graduate degree program in Aerospace Engineering and the Graduate Faculty of the University of Kansas in partial fulfillment of the requirements for the degree of Doctor of Philosophy.

Chairperson Richard Hale

Co-Chairperson Shawn S. Keshmiri

$\begin{array}{r}\hline \text { David Downing } \\ \hline \text { Mark Ewing } \\ \hline \text { Bryan Young }\end{array}$

Date Defended: November $25^{\text {th }}, 2013$ 
The Dissertation Committee for Katrina Legursky

certifies that this is the approved version of the following dissertation:

SYSTEM IDENTIFICATION AND THE MODELING OF SAILING YACHTS

Chairperson Richard Hale

Co- Chairperson Shawn S. Keshmiri

Date approved: November $27^{\text {th }}, 2013$ 


\section{Abstract}

This research represents an exploration of sailing yacht dynamics with full-scale sailing motion data, physics-based models, and system identification techniques. The goal is to provide a method of obtaining and validating suitable physics-based dynamics models for use in control system design on autonomous sailing platforms, which have the capacity to serve as mobile, long range, high endurance autonomous ocean sensing platforms. The primary contributions of this study to the state-of-the-art are the formulation of a five degree-of-freedom (DOF) linear multi-input multi-output (MIMO) state space model of sailing yacht dynamics, the process for identification of this model from full-scale data, a description of the maneuvers performed during on-water tests, and an analysis method to validate estimated models. The techniques and results described herein can be directly applied to and tested on existing autonomous sailing platforms.

A full-scale experiment on a $23 \mathrm{ft}$ monohull sailing yacht is developed to collect motion data for physics-based model identification. Measurements include 3 axes of accelerations, velocities, angular rates, and attitude angles in addition to apparent wind speed and direction. The sailing yacht herein is treated as a dynamic system with two control inputs, the rudder angle, $\delta_{R}$, and the mainsail angle, $\delta_{B}$, which are also measured. Over 20 hours of full scale sailing motion data is collected, representing three sail configurations corresponding to a range of wind speeds: the Full Main and Genoa (abbrev. Genoa) for lower wind speeds, the Full Main and Jib (abbrev. Jib) for mid-range wind speeds, and the Reefed Main and Jib (abbrev. Reef) for the highest wind speeds. The data also covers true wind angles from upwind through a beam reach.

A physics-based non-linear model to describe sailing yacht motion is outlined, including descriptions of methods to model the aerodynamics and hydrodynamics of a sailing yacht in surge, sway, roll, and yaw. Existing aerodynamic models for sailing yachts are unsuitable for control system design as they do not include a physical description of the sails' dynamic effect on the system. A new aerodynamic model is developed and validated using the full-scale sailing data which includes sail deflection as a control input to the system. The Maximum Likelihood Estimation (MLE) algorithm is used with non-linear simulation data to successfully estimate a set of hydrodynamic derivatives for a sailing yacht.

As there exists a large quantify of control algorithms which may be applied to systems described by a linear model, the non-linear model is simplified to a 5DOF MIMO state space model with a state vector including surge velocity, sway velocity, roll rate, yaw rate, and roll angle: $\vec{x}=\left[\begin{array}{lllll}U & V & P & R & \Phi\end{array}\right]^{T}$, and a control vector: $\vec{u}=\left[\begin{array}{ll}\delta_{R} & \delta_{B}\end{array}\right]^{T}$. Over 100 singlet and doublet maneuvers specifically designed to identify linear model dynamic responses are included in the full-scale data. The one-shot least squares (OSLS) 
technique offered a simple and fast means to estimate many linear models from this large sub-set of the full-scale data. As no sailing yacht linear dynamic model exists, especially for the test yacht, the only way to evaluate the fidelity of estimated models is to evaluate their predictive capability. This is accomplished through two separate criteria, Theil inequality coefficient $U_{T}<0.3$ and $R^{2}>0.75$, which are shown to provide sufficient quality models to enable control system design. Each linear model is estimated from only 3 maneuvers, one rudder singlet, one rudder doublet, and one sail singlet, and validated with a similar set of independently collected maneuvers. In total, 102 linear models are estimated in the Jib configuration, 17 linear models in the Genoa configuration, and 1 linear model in the Reef configuration.

The dynamic modes of the models estimated from the full-scale data are investigated using the eigenvectors and eigenvalues of the linear state space model $A$ matrix. First, the estimated models are characterized by the number of first and second order modes observed for each given model, and are referred to herein as Type A or Type B models. Type A models exhibit two second order modes, and one first order mode, whereas the Type B models exhibit one second order mode and three first order modes. The modes are then separated by natural frequency. A subset of models from the Jib configuration which exhibit an $R^{2}>0.88$ are analyzed via eigenvector modal analysis.

It is shown that all sailing yacht models will contain a second order mode (referred to herein as Mode 1A.S or 4B.S) which is dependent upon trimmed roll angle. For the test yacht it is concluded that for this mode when the trimmed roll angle is $\Phi>10^{\circ}$, roll rate and roll angle are the dominant motion variables, and for $\Phi<8^{\circ}$ surge velocity and yaw rate dominate. This second order mode is dynamically stable for $\Phi>12^{\circ}$. It transitions from stability in the higher values of $\Phi$ to instability in the region defined by $8^{\circ}<\Phi<12^{\circ}$. These conclusions align with other work which has also found roll angle to be a driving factor in the dynamic behavior of a tall-ship (Johnson, Miles, Lasher, \& Womack, 2009).

It is also shown that all linear models also contain a first order mode, (referred to herein as Mode 3A.F or 1B.F), which lies very close to the origin of the complex plane indicating a long time constant. Measured models have indicated this mode can be stable or unstable. The eigenvector analysis reveals that the mode is stable if the surge contribution is $<40 \%$ and the sway contribution is $>20 \%$.

The small set of maneuvers necessary for model identification, quick OSLS estimation method, and detailed modal analysis of estimated models outlined in this work are immediately applicable to existing autonomous mono-hull sailing yachts, and could readily be adapted for use with other wind-powered vessel configurations such as wing-sails, catamarans, and tri-marans. 


\section{Acknowledgements}

This dissertation would not have been possible without the incredible technical, financial, and emotional support of many people. I would first like to thank the Self Graduate Fellowship program for supporting me financially for four years which enabled me to pursue a research topic which was close to my heart and which I am very passionate about. The Fellowship also gave me invaluable skills in communication and leadership, a network of people outside my discipline, and a group of life-long friends. I am truly grateful for the vision of Al and Lila Self in establishing such a valuable fellowship program for graduate students at the University of Kansas.

I am sincerely grateful to Dr. Richard Hale for having the vision and patience to help me undertake this project. I have gone through a lot both personally and professionally throughout my time in graduate school, and I would not have made it without the sound advice and unwavering support I have received from him. He has supported both me and this project financially, always making sure I had the resources I needed to complete my work. I would also like to thank him for helping me become a more concise technical writer, for he has always been able to take my most confusing sentences and make them sound fabulous.

I am indebted to my co-advisor Dr. Shawn Keshmiri for taking me on as a graduate student and providing me with technical expertise and clarification throughout this project. As someone who already had more than a full work load when he began working with me, I truly appreciate all the time you devoted to thinking through and understanding my work. I especially enjoyed all the engaging discussions about how the boat and an airplane are alike and different.

Next I would like to thank Ted Kuwana for donating his Precision 23 Avanti for my research. Using her as a test platform inspired the much of the work I have accomplished and it would not have been possible without his generosity in donating her to my research. I'm also indebted to Avanti for being a pleasure to sail in any conditions and always carrying me safely back to the slip. In all the hours I spent single-handing aboard her, I never once experienced a serious breakdown or emergency situation.

I would like to acknowledge the work of Ryan Evans, Mickey Clemon, Chris Hedden, and Robert Burns for aiding in the development of the software for the data acquisition system. I 
never would have found my way through the ins and outs of the hardware/software interface without the expertise of these gentlemen.

And lastly, but most importantly, I would like to thank my friends and family who have given me unwavering support throughout this whole process. The friendship of Sommer Amundsen and Emily Arnold helping me get through all the life issues that would have been show stoppers if not for their support at those critical times when I needed support, whether it was babysitting or talking through a MATLAB script or just being a shoulder to cry on.

To my family: Dad, I could not have done this without all of your support. I'm not sure I can actually list all the ways you have helped me succeed in this endeavor, but I am going to try: helping me sand and paint the bottom of the boat, driving all the way to Lawrence from Chicago whenever I needed some extra help for whatever reason, sending money when money was too tight, buying me a laptop so I could work from home, spending hours on the phone helping me sort through my data and reassuring me that it made sense, for editing drafts of my papers, for going to a conference with me so I didn't have to leave Michael for a weekend when he was only 2 months old, for giving me the life experiences I needed to make this project a success, the list could go on. Mom, thanks for letting Dad be there to help me, and for always being there when I just needed someone to talk to. Grandpa, thank you so much for the financial support to get me through the last year, and for giving me the Gemperline sailing bug.

And to my husband, Brian, who has been there through all of it, the good, the bad, and the ugly. You are my best friend, and my calm in the storm. 


\section{Table of Contents}

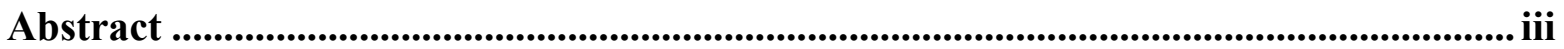

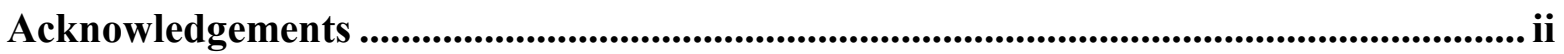

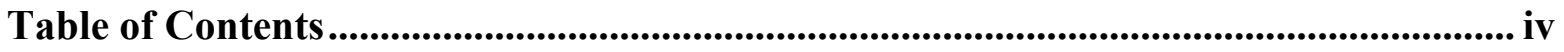

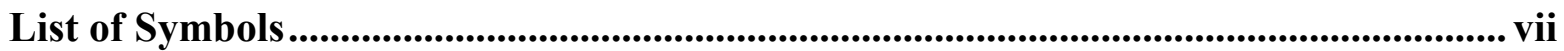

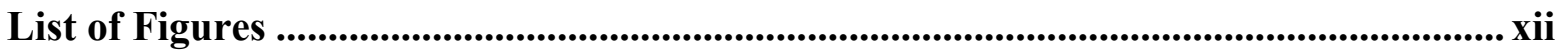

List of Tables...............................................................................................................

$1 \quad$ Introduction ......................................................................................................................... 1

1.1 Potential Applications for System Identification of Sailing Yacht Dynamics.......... 2

1.2 Organization of the Dissertation .......................................................................... 4

2 Existing Work in the Field ........................................................................................... 5

2.1 Methods of Sailing Yacht Performance Prediction ................................................. 5

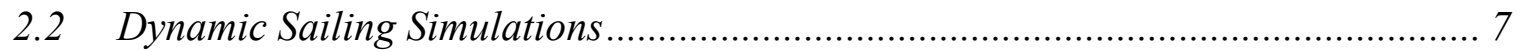

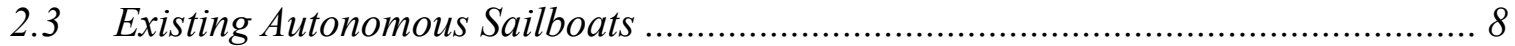

2.4 Modeling and Parameter Estimation for Ships ............................................... 9

3 Non-linear Dynamic Models of a Sailing Yacht ................................................. 11

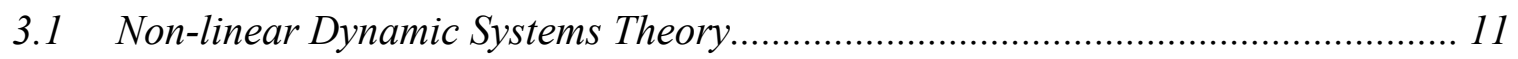

3.1.1 Coordinate Systems ................................................................................ 12

3.2 Four DOF Non-Linear Dynamic Model ......................................................... 14

3.3 Modeling Hydrodynamic Forces and Moments............................................. 15

3.3.1 Modeling with Hydrodynamic Derivatives ................................................. 16

3.3.2 Modeling with Delft Systematic Yacht Hull Series (DSYHS) ...................... 18

3.3.3 Rudder Forces and Moments ..................................................................... 18

3.4 Modeling Aerodynamic Forces and Moments ............................................... 20

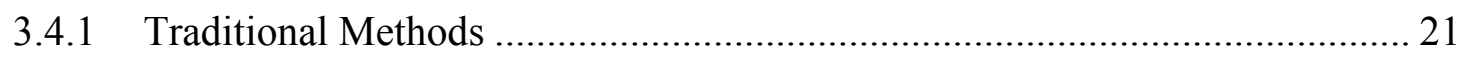

3.4.2 New Aerodynamic Force Model Including Sail Deflection .......................... 23

3.4.3 Validation of New Model with Full-scale Data............................................ 26

3.5 Implementation of Non-linear Model in MATLAB/Simulink .............................. 30

4 Linear Dynamic Sailing Yacht Models ......................................................................... 35

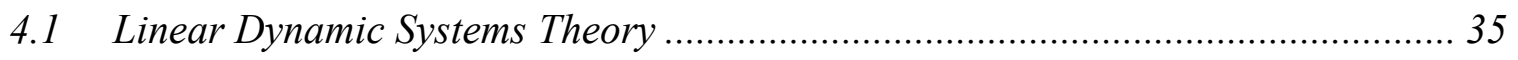




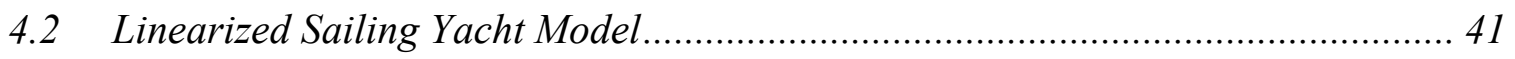

4.3 Numerical Linearization of 10m Sailing Yacht Based on Non-Linear Model ........ 43

5 Experimental Set-up .......................................................................................................... 47

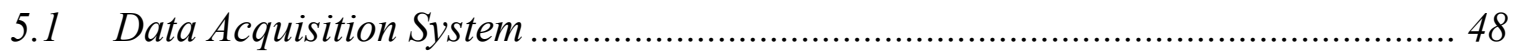

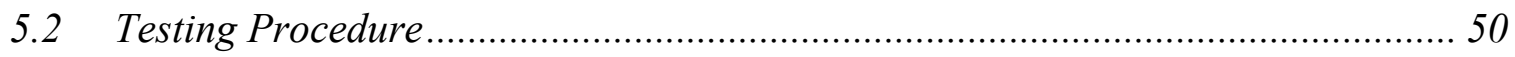

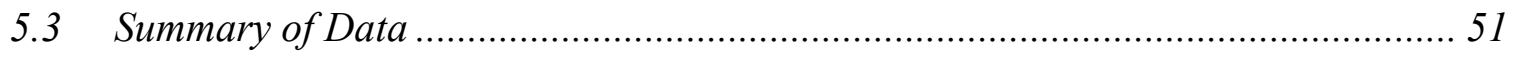

5.3.1 Full Main and Genoa (Genoa Configuration)............................................ 52

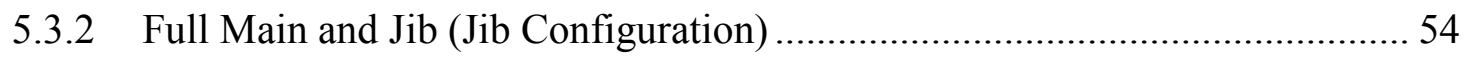

5.3.3 Reefed Main and Jib (Reef Configuration) ............................................. 55

6 System Identification........................................................................................................... 57

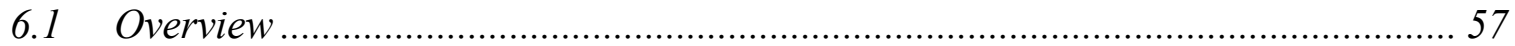

6.2 One Shot Least Squares Formulation ................................................................... 59

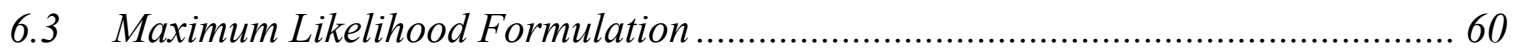

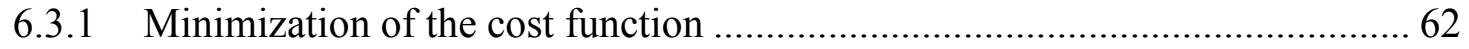

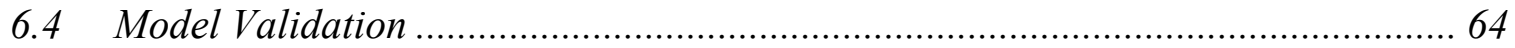

7 Non-Linear Model Estimation ................................................................................. 70

7.1 MLE Estimation of Non-Linear Hydrodynamic Derivatives ................................ 70

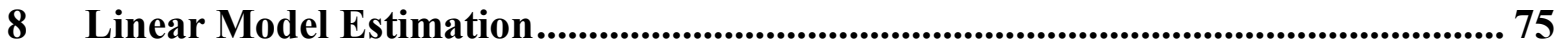

8.1.1 Models Estimated From Simulation Data ................................................... 76

8.2 Preparation of Full-scale Data for Linear System Identification........................... 77

8.3 Estimation of Linear Models from Full-Scale Data ........................................ 81

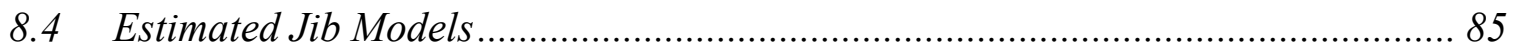

8.4.1 Modal Analysis for Estimated Jib Models ................................................. 85

8.4.2 Presentation of the Best Five Jib Models ................................................... 106

8.4.3 Best Estimated Jib Model ........................................................................... 108

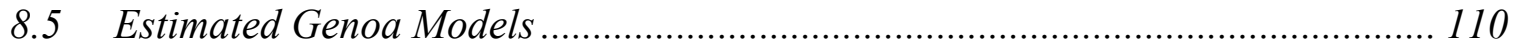

8.5.1 Presentation of Modes for Genoa Models ................................................... 110

8.5.2 Presentation of the Best Five Genoa Models............................................. 120

8.5.3 Best Estimated Genoa Model …................................................................ 121

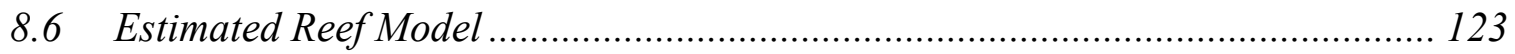

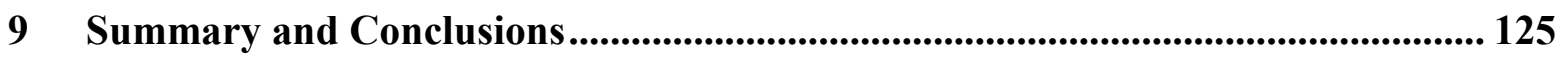

9.1 Summary of Contributions to the Field ........................................................ 125

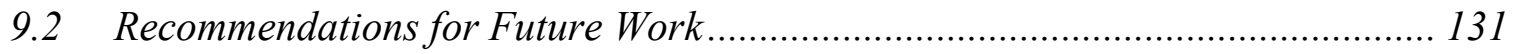


References............................................................................................................................................ 135

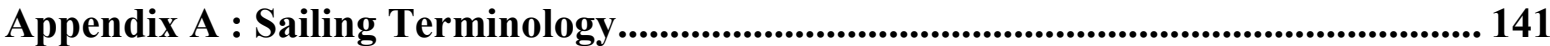

Appendix B : Delft Systematic Yacht Hull Series............................................................. 145

Appendix C: Linear Model Estimated from 6 ID Maneuvers....................................... 153 


\section{List of Symbols}

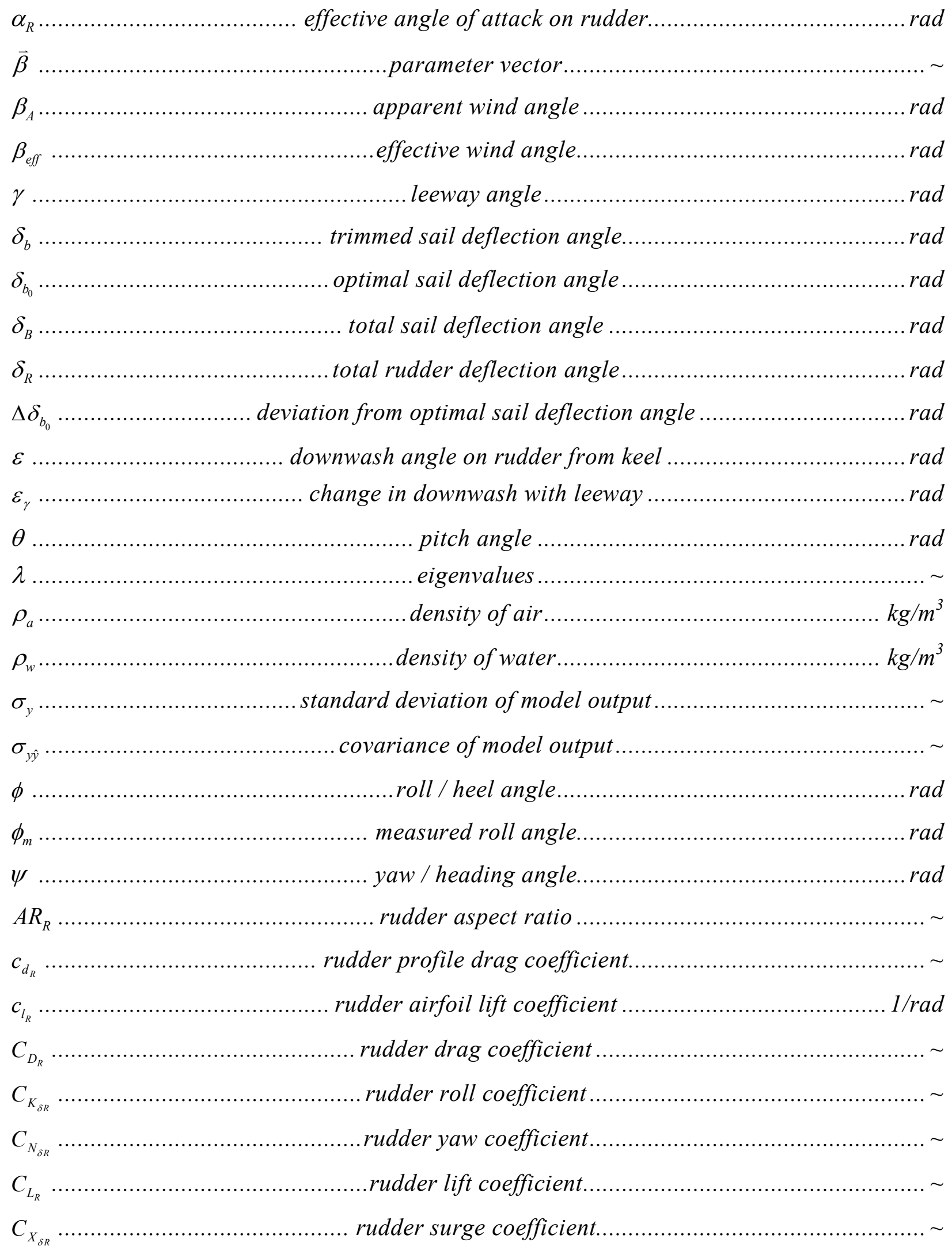




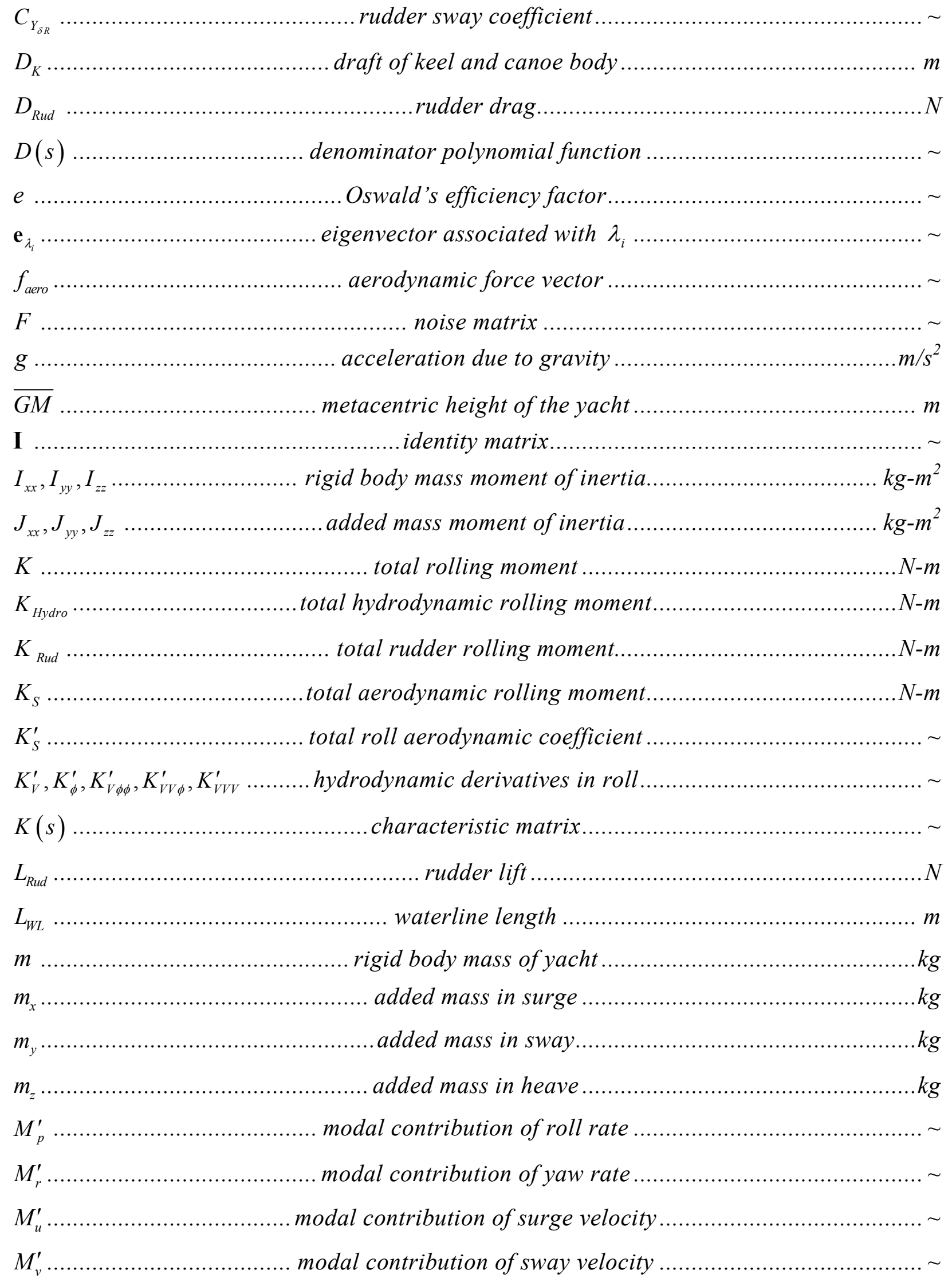

draft of keel and canoe body. ..rudder drag.

denominator polynomial function

Oswald's efficiency factor.

eigenvector associated with $\lambda_{i}$

aerodynamic force vector

noise matrix

acceleration due to gravity

metacentric height of the yacht

identity matrix.

rigid body mass moment of inertia.

added mass moment of inertia total rolling moment.

total hydrodynamic rolling moment.

total rudder rolling moment.

total aerodynamic rolling moment. total roll aerodynamic coefficient

hydrodynamic derivatives in roll. characteristic matrix.

rudder lift. .N .. waterline length rigid body mass of yacht..... $m$

.............................................. ri added mass in surge ..added mass in sway added mass in heave. modal contribution of roll rate modal contribution of yaw rate modal contribution of surge velocity. modal contribution of sway velocity

$$
m
$$
$N$ $\sim$ 


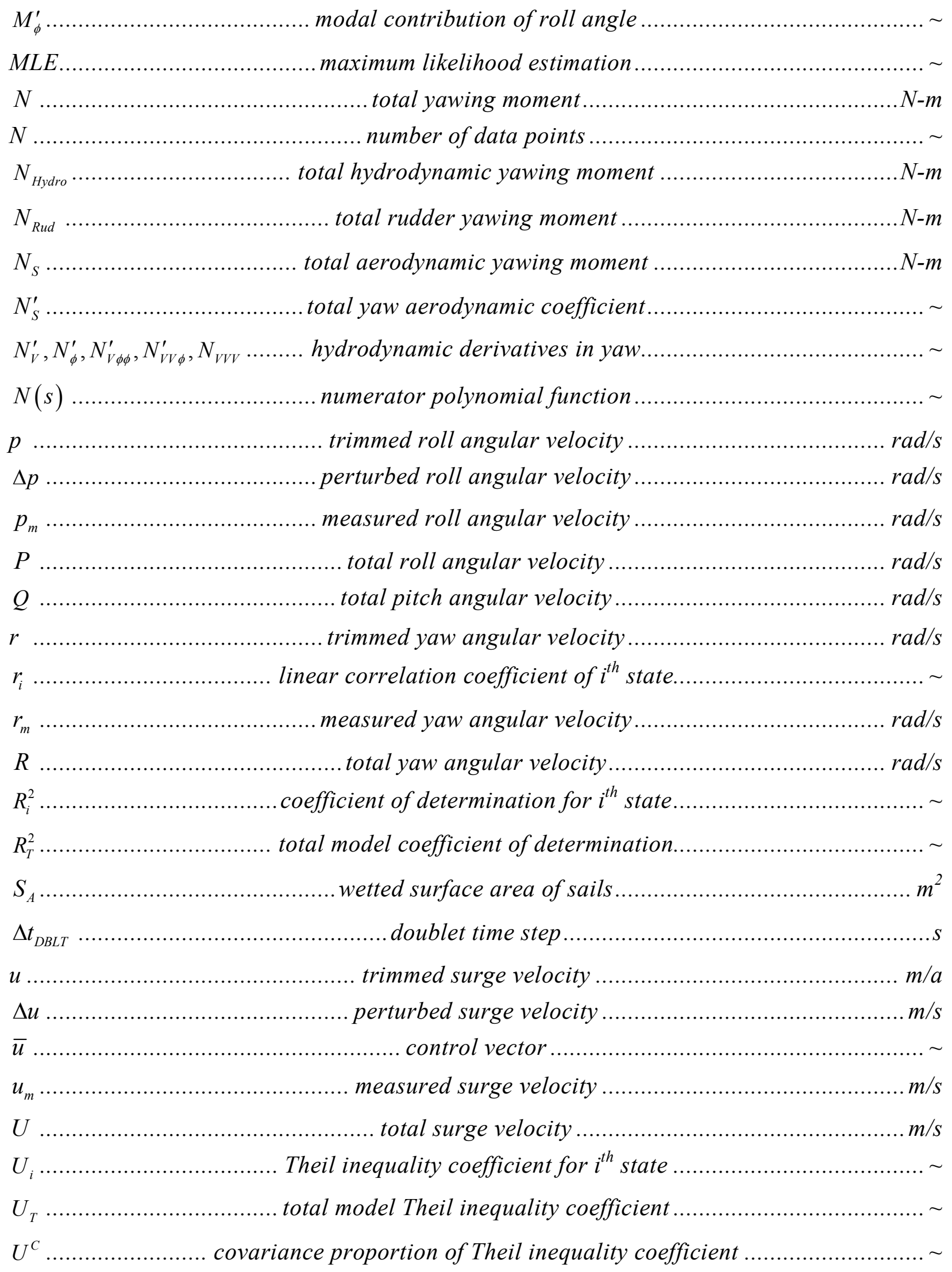



total sway velocity. $\mathrm{m} / \mathrm{s}$ non-dimensional sway velocity .. $\sim$ noise vector.

$X_{m}$ state measurement vector total surge rudder force. $N$

$X_{S}$ total surge aerodynamic force.... N

$X_{S}^{\prime}$ total surge aerodynamic force coefficient

$X_{S_{0}}^{\prime}$ optimal sail coefficient in surge. sail derivative in surge

$X_{V V}^{\prime}, X_{\phi \phi}^{\prime}, X_{V R}^{\prime}, X_{V V V V}^{\prime}$ hydrodynamic derivatives for surge output vector.

$y_{i}$ measured model output of $i^{\text {th }}$ state $\hat{y}_{i}$ estimated model output of $i^{\text {th }}$ state 
$\bar{y}_{i}$

$\hat{\bar{y}}_{i}$

$y_{b}$

$Y$

$Y$

$Y_{R u}$

$Y_{S}$

$Y_{S}^{\prime}$

$Y_{V}^{\prime}, Y_{\phi}^{\prime}, Y_{V \phi \phi}^{\prime}, Y_{V V \phi}^{\prime}, Y_{V V V}^{\prime}$

$z_{b}$

$z_{\mathrm{COE}}$ $z_{R}$ mean of measured model output of $i^{\text {th }}$ state mean of estimated model output of $i^{\text {th }}$ state lateral location of c.g. of yacht total sway force. total sway hydrodynamic force. total sway rudder force. total sway aerodynamic force total sway aerodynamic force coefficient. hydrodynamic derivatives for sway vertical location of c.g. of yacht

$m$ vertical location of aerodynamic center of effort $m$ vertical location of rudder center of effort......$$
m
$$ 


\section{List of Figures}

Figure 1: Body Coordinate System with Linear Velocities .................................................... 13

Figure 2: Breakdown of total resistance forces on a sailing yacht hull. ...................................... 16

Figure 3: Definition of rudder deflection. All the angles shown are positive............................... 19

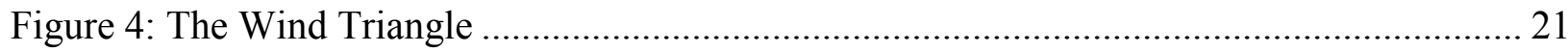

Figure 5: Typical sailing yacht force and moment coefficients shown as functions of effective

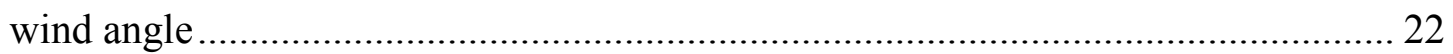

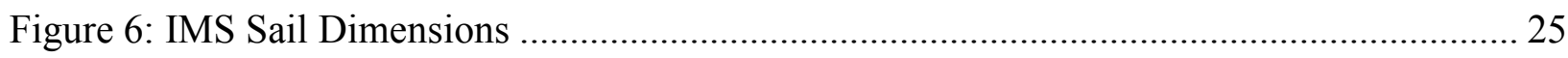

Figure 7: Linear fit from averaging 10 minutes of trimmed sailing data to estimate the optimal sail angle curve for the Precision 23 ................................................................. 26

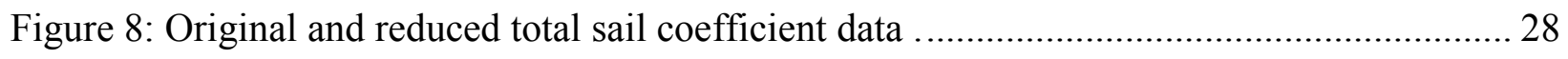

Figure 9: Measured optimal sail coefficients in 4 DOF for Avanti............................................. 29

Figure 10: Measured aerodynamic derivatives for Avanti ....................................................... 30

Figure 11: Simulink block diagram of non-linear simulation.................................................... 33

Figure 12: Measured and simulated response to a rudder singlet............................................... 34

Figure 13: The complex plane showing stable first order mode at -0.5 and an unstable first order

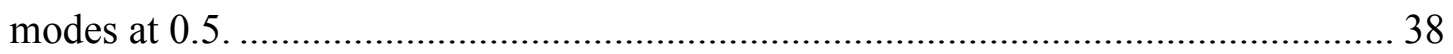

Figure 14: Time response of both stable and unstable first order modes. ................................... 39

Figure 15: Complex plan showing a stable second order mode at $-0.5+/-0.5 \mathrm{i}$ and an unstable

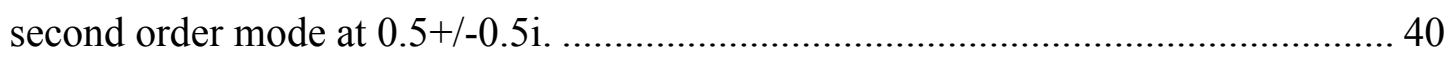

Figure 16: Time response of both stable and unstable second order modes.................................. 41

Figure 17: Sample Polar Diagram for Sailboat (Gentry, 1995) ……………………............... 42

Figure 18: Linear model response to rudder singlet at varying true wind speeds. ....................... 45

Figure 19: Linear model response to rudder singlet at varying true wind directions. ................... 46

Figure 20: Precision 23 geometry reverse-engineered using photogrammetry to develop a computer-aided design (CAD) model.................................................................... 47

Figure 21: Avanti under sail on Clinton Lake in the Reefed Main and Jib sail configuration...... 48

Figure 22: Data flow diagram of data acquisition system. ......................................................... 49

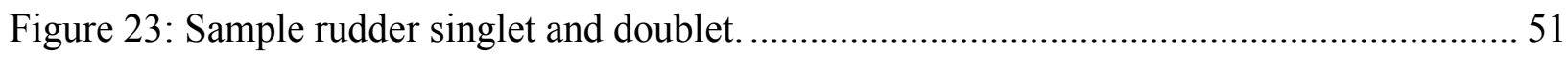

Figure 24: Example no roll response to boom singlets in a set of Genoa data ............................ 53

Figure 25: Example of a clear dynamic response in surge, sway, and yaw to rudder singlets, but no response in roll in a set of Genoa data.............................................................. 53

Figure 26: Diagram of MLE and iterative LS estimation structure ............................................ 58 
Figure 27: (a) Example of highly correlated model fit with $R^{2}$ of 0.91 (b) Example of poorly

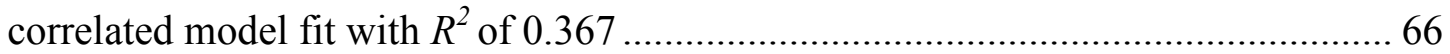

Figure 28: Example of what normalized data looks like for calculation of $R^{2}$.......................... 67

Figure 29: Rudder input sequence used in non-linear simulator to generate estimation data. ..... 71

Figure 30: Time history of simulated and estimated non-linear hydrodynamic model ................. 74

Figure 31: Comparison of the response of the estimated linear model to the 'Measured' non-

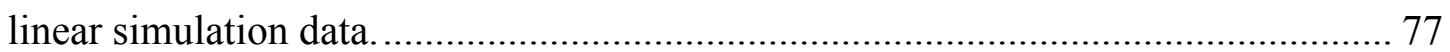

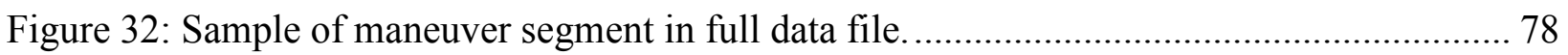

Figure 33: Sample set of smoothed maneuver data sets for (a) a rudder and sail input and (b) for

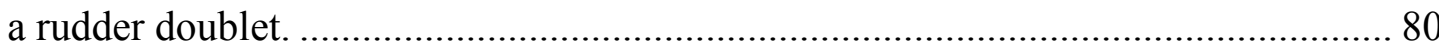

Figure 34: Sample set of ID data for the Genoa configuration with one boom singlet, one rudder

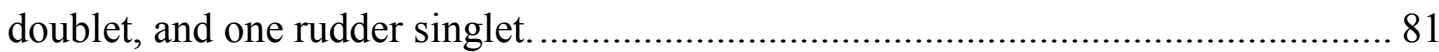

Figure 35: Sample set of ID data for the Jib configuration with one sail singlet, one rudder

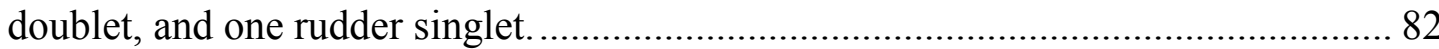

Figure 36: Breakdown of linear models by boat configuration, model type, modal order, and natural frequency. 83

Figure 37: All Jib Type A Modes plotted on the complex plane before being sorted by natural frequency.

Figure 38: Jib Type A Modes for models with an $R^{2}>0.88$ fit to the ID data which will be utilized for detailed eigenvector modal analysis........................................................ 88

Figure 39: Definition of stability regions in the complex plane. ……………………………..... 89

Figure 40: Jib Modes 1A showing a clear trend that this mode is dependent upon roll angle..... 90

Figure 41: Contribution of State Variables to Mode 1A according to trimmed roll angle........... 91

Figure 42: Second order Modes 2A colored according to trimmed roll angle. ............................ 92

Figure 43: Contributions of each state to Mode 2A according to trimmed roll angle.................. 93

Figure 44: Modes 3A according to trimmed boat velocity......................................................... 95

Figure 45: Contributions of each state to Mode 3A with respect to U......................................... 96

Figure 46: Mode 4B colored according to trimmed roll angle. …………………………............ 97

Figure 47: Contribution of each state to Mode 4B according to trimmed roll angle.................... 97

Figure 48: Modes 1B colored according to boat velocity, clearly clustered to the far left of the

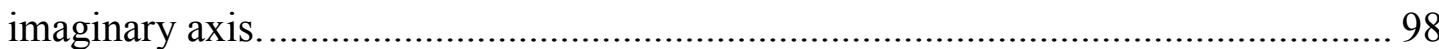

Figure 49: Contribution of each state to Mode 1B according to U.............................................. 99

Figure 50: Mode 2B colored according to apparent wind angle................................................... 99

Figure 51: Contribution of each state to Mode 2B according to U........................................... 100 
Figure 52: Mode 3B colored according to apparent wind angle......................................... 101

Figure 53: Contribution of each state to Mode 3B according to U....................................... 101

Figure 54: Distribution of trimmed boat speeds, roll angles, apparent wind speeds and angles for the estimated Type A Jib Models.

Figure 55: Distribution of trimmed boat speeds, roll angles, apparent wind speeds and angles for the estimated Type B Jib Models.

Figure 56: Breakdown of the best $5 \mathrm{Jib}$ models by state showing difficulty matching the roll degree of freedom of the TEST data. 106

Figure 57: Theil error proportions for the best $5 \mathrm{Jib}$ models reported for the entire model fit, as well as individually by state showing high bias proportions in yaw.

Figure 58: Best Jib Model Fit to the ID Data........................................................................ 109

Figure 59: Best Jib model fit to the test data.

Figure 60: Genoa Modes 1A showing a trend with roll angle as there was with Jib Mode 1A. 112

Figure 61: Genoa Modes 1A showing a trend with apparent wind speed. 112

Figure 62: Genoa Mode 2A showing the lower roll angles clustered farther to the left of the complex plane and having damping less than 0.3

Figure 63: Genoa Mode 2A showing a the higher wind speeds correspond very well to the higher roll angles of Figure 62. 113

Figure 64: Genoa Mode 1A colored according to apparent wind direction. 114

Figure 65: Genoa Mode 2A colored according to apparent wind direction. 114

Figure 66: Modes 3A clustered to the right of the imaginary axis and centered at 0.034 , indicating instability.

Figure 67: Genoa Mode 1B centered at -0.158 , indicating stability. 115

Figure 68: Genoa Mode 2B centered very close to the origin at -0.006 possibly indicating neutral stability of this mode.

Figure 69: Genoa Mode 3B centered to the right of the imaginary axis at 0.094 indicating instability. 116

Figure 70: Genoa Mode 4B according to roll angle. 117

Figure 71: Genoa Mode 4B according to apparent wind speed.

Figure 72: Distribution of trimmed boat velocity, roll angle, apparent wind speed and apparent wind angle for estimated Genoa Type A models.

Figure 73: Distribution of trimmed boat velocity, roll angle, apparent wind speed and apparent wind angle for estimated Genoa Type B models. 119

Figure 74: Breakdown of the best 5 Genoa models fit by state. 120 
Figure 75: Theil error proportions for the best 5 Genoa models reported for the entire model fit,

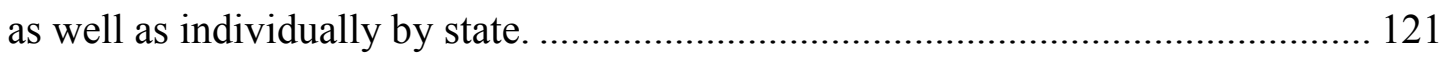

Figure 76: Best Genoa model fit to the ID data............................................................. 122

Figure 77: Best Genoa model fit to the TEST data........................................................... 122

Figure 78: Data used to estimate the only Reef model. ...................................................... 123

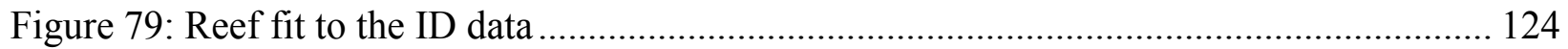

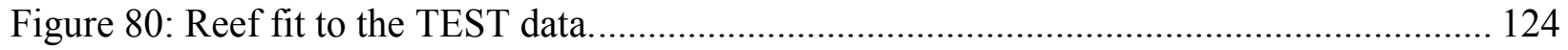

Figure 81: Basic Parts of a Monohull sailing Yacht ............................................................ 141

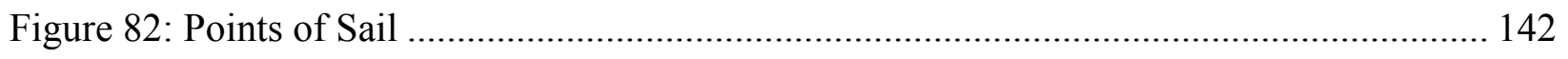

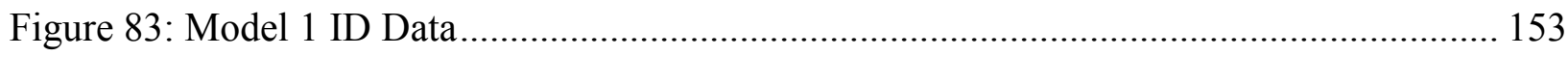

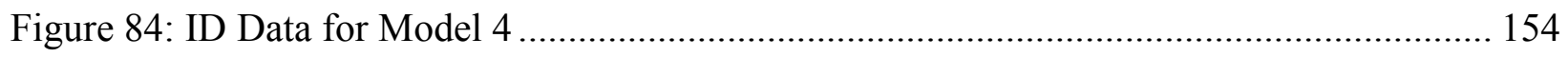

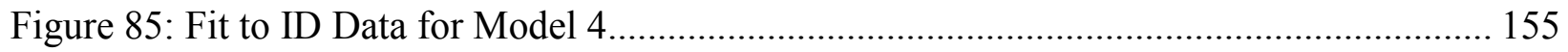

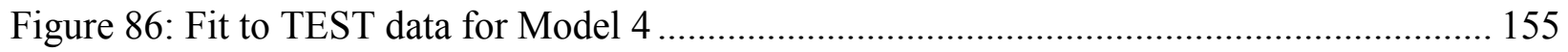

Figure 87: Statistical Result of Estimation of Models 1 - 4 ............................................... 156

Figure 88: Breakdown of Model fit statistics into states for Models 1-4 .............................. 157

Figure 89: Breakdown of Theil inequality coefficient into bias, variance, and covariance proportions for the ID data fit ........................................................................ 158

Figure 90: Breakdown of Theil inequality coefficient into bias, variance, and covariance proportions for the TEST data fit. 


\section{List of Tables}

Table 1: List of Velocities in Body Fixed Coordinate Frame..................................................... 13

Table 2: Parameters for 10m Racer/cruiser simulation............................................................. 32

Table 3: Summary of maneuvers collected in the Genoa configuration........................................ 54

Table 4: Summary of maneuvers collected in the Jib configuration. ......................................... 55

Table 5: Summary of suitable maneuvers collected in the Reef configuration .............................. 55

Table 6: Summary of MLE Estimation of Hydrodynamic Derivatives ........................................... 73

Table 7: Difference in the average magnitude of Modes 1A and Modes 2A ............................... 93

Table 8: Comparison of the ranges represented by the Type A and Type B models in the Jib

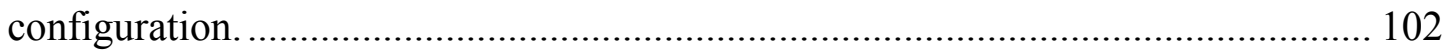

Table 9: Comparison of the mean boat velocity, roll angle, apparent wind speed and apparent wind direction for the estimated Type A and Type B models.................................. 102

Table 10: Difference in ID and TEST data trimmed surge velocities ...................................... 108

Table 11: Comparison of the ranges represented by the Type A and Type B models in the Genoa configuration.

Table 12: Comparison of the mean boat velocity, roll angle, apparent wind speed and apparent wind direction for the estimated Type A and Type B models................................. 118

Table 13: Summary of total valid linear models estimated from full-scale data......................... 128

Table 14: Mean locations of all real roots for Jib and Genoa................................................... 129

Table 15: DSYHS Coefficients for Residuary Resistance of Cano Body ................................... 147

Table 16: DSYHS Coefficients for Residuary Resistance of Keel............................................. 147

Table 17: DSYHS Coefficients for Change in Wetted Surface Area with Heel ........................ 148

Table 18: DSYHS Coefficients for Added Residuary Resistance due to Heel............................ 148

Table 19: DSYHS Coefficients for Added Residuary Resistance due to Heel of the Keel........ 149

Table 20: DSYHS Coefficients for Surge Force from Leeway ................................................. 149

Table 21: Coefficients for Induced Drag of Keel in Leeway................................................... 150

Table 22: DSYHS Keel and Rudder Characteristics for the Precision 23 ………..................... 151

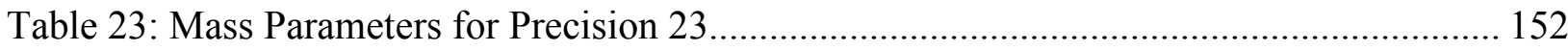

Table 24: DSYHS Hull Parameters for Precision 23 …….................................................... 152 


\section{Introduction}

A yacht under sail is a beautifully complex system ${ }^{*}$. Under the skills of an expert crew and helmsman the boat is placed in a delicate balance of forces that allow it to move gracefully through the water without burning an ounce of fuel. Building an autonomous controller for such a vessel is a challenge because of that very complexity. Where a seasoned sailor gets his insight into the operation of his sailboat from a wealth of past experience and sensory inputs, a computer has only the model given to it and the information available from its sensors to make the same decisions. Translating this wealth of experience to a computer controller which is capable of autonomous operation is a formidable problem.

Autonomous sailboats have great potential to serve as platforms for long range and duration oceanic observing. Current ocean sensing platforms in use include autonomous underwater vehicles (AUV's), remotely operated underwater vehicles (ROV's), powered autonomous surface vessels (ASV's), floating or anchored buoys, and manned surface vessels. A sailboat has some distinct advantages over the existing sensing platforms. The biggest advantage is the inherent ability to move without requiring a powered propulsion source because it uses the wind. Thus, it can perform longer duration missions and go farther from home than vehicles which are limited by either battery life or fuel storage for operation. Because its size is not limited by the power and containment of the propulsion system, the available space in the hull and stored or harvested power for payload can be dedicated to the payload. The sailing yacht is also very acoustically quiet in the water compared to vessels propelled by rotating machinery and propellers, making it a more ideal platform for towed sensor arrays. For a more in depth discussion of possible applications for an autonomous sailing yacht see (Cruz \& Alves, 2008). Fully autonomous sailing systems are currently under development, but to date have not been robust enough to handle the long duration deployments that will make them successful sensing platforms.

System identification is a powerful tool that allows engineers to use real data to build models which can later be used for control system design. These techniques have been widely explored by the aerospace industry. They have been used to classify an aircraft's handling

\footnotetext{
* If the reader is unfamiliar with sailing terminology, a glossary of terms and some useful diagrams may be found in Appendix A.
} 
qualities, develop controllers for autonomous flight systems, improve physics-based simulation models, and to compare wind tunnel vs. flight test measurements (Jategaonkar, 2006; Morelli \& Klein, 2005; Tischler \& Remple, 2006). These ideas have great potential for increasing the fidelity of physics-based sailing yacht models through full-scale sea trial data, but have yet to be explored. Sailing yachts are particularly difficult systems to model from a controls perspective because they rely on the uncontrollable and dynamically variable wind for propulsion. This project is a thorough investigation of sailing yacht dynamics through simulation and full-scale test data using system identification techniques to identify a model appropriate for advanced control system design.

\subsection{Potential Applications for System Identification of Sailing Yacht Dynamics}

There are two potential major applications of this work, the first being to improve control system design for autonomous sailing yachts, the second being to characterize the non-linear parameters of a sailing yacht based on full-scale measurements.

The primary motivation for this project was to create a robust autonomous sailing system to serve as an ocean sensing platform. Design of autonomous sailboats has primarily been for competitions; and thus often the entrants are groups of students. Competition boats have been successful at navigating a small race course and performing certain maneuvers, but have not yet produced a vehicle robust enough to cross oceans. Control system development of these boats has been very simple, and in general closely resembles a standard sailboat autopilot controlling rudder and heading. No advanced modeling or control strategies have been utilized on the vehicles. To date there has been very little technical literature published on the subject of advanced modeling and control for an autonomous sailing yacht, particularly studies which have utilized real sailing data. The physics-based model is the foundation upon which almost all autonomous aircraft development has been achieved, allowing implementation of advanced control strategies. A combination of a viable physics-based model and the ability to identify parameters in that model will greatly contribute to the development of advanced control systems for both existing and future autonomous sailing vessels.

A secondary motivation for this project is to advance the field of study of sailing yacht dynamics. Most of the scientific research for sailing yachts has been motivated by professional racing teams, and as such is performance oriented. A great deal of work has gone into studying 
steady state yacht motion as will be shown in Section 2.1, but very little has been done with fullscale motion data. The ability to classify the non-linear sailing yacht parameters from a relatively simple set of measurements that have been taken aboard this project's test vehicle is highly appealing to the community of people who study sailing yacht dynamics. Yacht designers can use the information gained by parameter estimation from full-scale data to validate their designs as well as the performance and maneuvering capability of the yacht. This technology can also be used to generate high fidelity simulations for training and analysis purposes. The most recent, $34^{\text {th }}$ edition, of the America's Cup was raced in 72 foot hydro-foiling catamarans with wingsails, vehicles that looked and behaved much more like aircraft with important dynamics than steady state sailing yachts. As the America's Cup is the largest driver of innovation in sailboat technology, studies of sailing yacht dynamics such as the one presented here will become increasingly important to competitive racing teams.

Based on a thorough literature review, summarized herein in Chapter 2, it has been determined that the open literature does not provide sufficient knowledge for capturing the physics of sailing yacht dynamics necessary for advanced control system design. In particular, no attempt has been made to utilize a linearized sailing yacht dynamic model. Also, as will be shown in Section 2.2, the use of full-scale data to validate existing nonlinear physics-based models has been limited, and the few examples do not include the sails as inputs to the dynamic system.

The test vehicle used to collect data for this project is ideal for a study of sailing yacht dynamics in the sense that it is a small enough sailing yacht to exhibit some of the more complex dynamic behaviors associated with sailing in different conditions, yet it is large enough to carry a person to actually pilot and move around the vehicle without inherently changing the dynamics. As such, the results of the project will be readily applicable to monohull sailing yachts with weighted keels of varying sizes and shapes. The techniques and conclusions will also provide a framework for modeling and system identification of yachts with different configurations such as catamarans and yachts with solid wings rather than sails. The monohull yacht with flexible sails is the most complex configuration, so moving towards the other configurations should be a simplified abstraction of the work presented herein. 


\subsection{Organization of the Dissertation}

This work begins with a survey of the field of sailing research and technology in Chapter 2, including more specifics about existing autonomous sailboat technology as well as a brief discussion of how system identification has been utilized in the context of ships and naval architecture. Chapter 3 presents the details of non-linear sailing yacht dynamic models including both hydrodynamic and aerodynamic force models, and a detailed description of the non-linear simulation which has been used to generate data to test identification algorithms. Chapter 4 simplifies the non-linear model to linear state space form. This Chapter includes a brief introduction to the theory of linear system dynamics, then presents the linear sailing yacht model structure which is the primary contribution of this dissertation. Chapter 5 discusses the full-scale experimental set-up including a description of the onboard electronics, an explanation of the maneuvers performed on the water, and details about the different sail configuration in which data has been collected. Chapter 6 provides an introduction to system identification and the details of the one-shot and maximum likelihood estimation (MLE) algorithms that have been used to identify sailing yacht dynamic models. Also included in Chapter 6 is a discussion of the way estimated models are evaluated and validated. Chapter 7 gives the results of the estimation of non-linear hydrodynamic derivatives from simulation data using MLE. Most important is Chapter 8, which details the results of linear system identification from the full-scale data. This Chapter may be considered the greatest contribution to the field of sailing yacht dynamics. The dissertation concludes with Chapter 9 which includes a summary and discussion of the primary results, as well as a substantial section of recommendations for future work in this field. 


\section{Existing Work in the Field}

\subsection{Methods of Sailing Yacht Performance Prediction}

The complexity of the yacht dynamic system has made accurate modeling a difficult task for yacht designers and engineers. Research into this field has mostly been driven by a racer's need to understand exactly what kind of performance his yacht is capable of attaining. The earliest work in the field (Letcher Jr, 1974) suggested that the handicap system used for racing yachts should include a system in which multiple ratings are assigned to one yacht based on the conditions she sails in during a particular race. This indicates that the performance capabilities of a yacht are weather and sea dependent.

This work was expanded upon by development of the yacht velocity prediction program (VPP) (Kerwin, 1975). The original program was a two degree of freedom (2DOF) solver which balanced the driving/resistance forces and the heeling/righting moments. Kerwin's VPP assumes a yacht is sailing in steady conditions, meaning constant wind speed and direction and no waves. This is still the case with most VPPs, however, current research is being focused on the development of VPP's that take into account the performance of a yacht in unsteady conditions (Azcueta, 2002; Fossati \& Muggiasca, 2010; Graf, Pelz, Bertram, \& Soding, 2007; Richardt, Harries, \& Hochkirch, 2005).

The inputs for a typical VPP are the geometrical characteristics of the yacht, and experimentally derived aerodynamic and hydrodynamic coefficients. One of the difficulties in modeling a yacht is the lack of a direct method for determining these coefficients without extensive experimentation. At the time the first VPP's were introduced, the primary method of dealing with this problem involved extensive tests in wind tunnels and towing tanks. The Delft Systematic Yacht Hull Series, or DSYHS, provides the most extensive database of such tests performed on yacht hull forms (Gerritsma, Moeyes, \& Onnink, 1977). This is an ongoing series consisting of parent models of yacht hulls whose geometry is systematically varied and towing tank tests performed on each variation. This series is currently the basis for rating ocean racing yachts which have not directly been analyzed in towing tank tests. Recent research is looking into methods of directly running a VPP from experimental results (Graf \& Bohm, 2005; H. Hansen, Jackson, \& Hochkirch, 2003). 
Towing tank and wind tunnel tests are expensive, especially when the data is desired to compare boats during the design process. Computational Fluid Dynamics, or CFD, is a numerical tool which is increasingly being implemented to obtain the forces acting on a yacht. CFD codes have been used to separately analyze the aerodynamic characteristics of the rig (Ciortan \& Guedes Soares, 2007; Masuyama, Tahara, Fukasawa, \& Maeda, 2009; Miyata \& Lee, 1999; Yoo \& Kim, 2006), and the hydrodynamic characteristics of the hull (Azcueta, 2002; Kim et al., 2009; Miyata, Akimoto, \& Hiroshima, 1997). These codes are generally run assuming steady conditions. Only recently have these numerical models been applied to sail aerodynamics in dynamic conditions (Augier, Bot, Hauville, \& Durand, 2013; Fossati \& Muggiasca, 2010) or to a maneuvering sailing yacht (Akimoto, Kim, Park, \& Jeong, 2012).

Progress is being made towards a VPP which directly incorporates both the aerodynamic and the hydrodynamic solvers (Roux et al., 2002). One of these combinations solves for equilibrium conditions by fixing the aerodynamic forces and numerically computes the hydrodynamic forces (Jacquin, Roux, Guillerm, \& Alessandrini, 2005). These kinds of numerical solvers have also been coupled with rigid body dynamic system models. In such a system, at every time step the forces and moments calculated from the CFD code are sent to a 6DOF system, where the new position and velocities of the yacht are calculated (Parolini \& Quarteroni, 2007). The only application to date of this capability has been evaluate and choose a keel bulb design for an America's Cup yacht. The use of CFD for performance prediction is moving towards applications for the next generation of high performance yachts which will to operate at much higher Froude numbers than traditional sailing yachts (Graf, Boehm, \& Renzsch, 2009).

The most common use of full-scale data to validate VPP programs and numerical codes has been to capture sail shapes while sailing full-scale for use in CFD codes. In (Masuyama et al., 2009) the authors present a complete analysis of measured full-scale sail shape and force data versus numerically calculated data for sails in the upwind conditions. They used CCD cameras to measure the real flying shape of the sails, and a dynamometer frame connected to the rigging and sail controls by load cells to measure the aerodynamic forces. The data collected from their fullscale sea trials was compared to numerical results calculated using the sail shapes captured by the CCDs. It was reported that overall the numerical results agreed well with the measured data. Recently a study has been done with actual pressure taps on a downwind sail which and has also 
validated the use of CFD codes (Viola \& Flay, 2012). Thus, numerical models of the sails are a good place to start when trying to predict the sail forces in the upwind condition. The analysis was not carried out for other trim states.

\subsection{Dynamic Sailing Simulations}

Yacht dynamics research has been focused on predicting the performance of a yacht in the design stage. The current methods are rooted in controlled tests and numerical codes as discussed in Section 2.1. Validation of these methods by full-scale sea trial data has been limited.

A modular simulation tool has been developed for simulating yacht motion where all the forces and moments are defined by separate files (Richardt et al., 2005). This tool can act as a standard VPP, but it can also operate in a dynamic mode where it predicts yacht motions. There was good correlation with full-scale sailing tests, but details of these tests are not specified. The main focus of this software has been its use as a VPP program. It has also been implemented into wind tunnel tests at the University of Auckland (H. Hansen et al., 2003). Integration of the software allowed users to utilize the real time measured aerodynamic forces and moments and predicted hydrodynamic forces and moments to dynamically trim the boat in the wind tunnel for maximum speed. Again, the focus of this kind of work is analysis of yacht performance in the design stage to ideally develop a faster boat.

The closest implementation of system identification techniques to a sailing yacht was done to develop a simulation to use as a training tool for sailors (Binns, Hochkirch, De Bord, \& Burns, 2008). The simulation was focused on evaluating the performance of a single iteration of an America's Cup yacht in the interest of evaluating the impact of decisions made during the start of a match race. Their steady state model is based upon extensive towing tank tests and wind tunnel experiments of the yacht which they are modeling. The dynamic parameters are then estimated after a series of full-scale tests with the boat performing specific maneuvers. This work was very interesting because in the starting sequence of a match race, the yacht undergoes extensive maneuvering, including stopping the boat head to wind and rapid tacking and continual course changing done in a small amount of space. The simulation results were satisfactory for their purpose in putting together a simulator for training, but the authors recommend better instrumentation and studies of the dynamic effect of the sails be included in further research of this type. 


\subsection{Existing Autonomous Sailboats}

In the last several years there has been a growing interest in designing autonomous sailboats for competitions such as the World Robotic Sailing Championship, SailBot, and the Microtransat Challenge. The focus of competitors in these events has been the design of a complete autonomous sailing system, including hull and rig design (Miller, Brooks, \& Hamlet, 2009; Rynne \& von Ellenrieder, 2010), sensors (Neal, Sauze, Thomas, \& Alves, 2009), onboard processing (Alves, Ramos, \& Cruz, 2008), navigation, and control (Stelzer, Proll, \& John, 2007). Models and control systems for existing boats have been similar to a standard autopilot driving a heading and choosing a sail angle based on wind angle. Only recently have physics-based models been presented for future use on autonomous sailing platforms (Petres, Romero-Ramirez, Plumet, \& Alessandrini, 2011; Xiao \& Jouffroy, 2011), however they do not include sails as inputs to the system. These models also have not been tested with or compared to full-scale sailing data. The most recent winner of the World Robotic Sailing competition has a control system that continually adjusts the sail angles until the boat speed is maximized. In order to test whether boat speed has increased, the sails can only be adjusted about every $10 \mathrm{~s}$, and with no model underneath the control system, it takes a while to achieve the right boat speed starting from the sails far out of trim.

There has been a fairly significant amount of work done in the area of autonomous sailboat navigation and path planning. Of particular interest are the tacking and jibing maneuvers. Fuzzy logic controllers have proved to perform these maneuvers well on real sailing platforms (Stelzer et al., 2007). Theoretical descriptions of ways to model and handle the no-go upwind zone for navigation have been presented but not implemented on full-scale boats as the actual autonomous sailboats in operation now do not use the physics based models in their control system design (Petres et al., 2011; Yang, Xiao, \& Jouffroy, 2011).

To the author's knowledge, none of the physics based models for control listed here have been tested or validated by experimental data. All current work in the open literature in advanced modeling and control of sailing yachts has been done in simulation with artificial models.

The only application of linear system identification to a wind powered boat is an example of Observer/Kalman identification for an autonomous wing sailed catamaran dynamic model for control purposes (Elkaim, 2002, 2009). The state space model was a 3DOF model whose states 
are the cross track error, the heading, and the angle of the rudders, again representing more of a standard autopilot model. The identification tests were performed under the power of a trolling motor and did not include the dynamics of the wing sail. The estimated model was used to design a Linear Quadratic Gaussian (LQG) controller. The controller performed well during tests on the full-scale boat. This model worked well because the wing sail does not produce the complicating effects of heel that a monohull yacht with traditional sails experiences. The controller did not include active control of the wing as part of the model, rather, the wing itself was designed to be self-trimming. Dr. Elkaim currently works with Harbor Wing Technologies, a company developing a prototype autonomous wing sailed catamaran.

\subsection{Modeling and Parameter Estimation for Ships}

The field of ship maneuvering and naval architecture has used system identification techniques to develop non-linear steering and maneuvering models since the early 1970's. These models are typically developed for large ships which do not generally experience a large amount of rolling motion, thus it is most common for estimated models to only have 3 DOF: surge, sway, and yaw. Several methods have been used to estimate these models including maximum likelihood (Abkowitz, 1980), extended Kalman filter (Abkowitz, 1980; Fossen, Sagatun, \& Sørensen, 1996), estimation-before-modeling (Yoon \& Rhee, 2003), and frequency domain methods (Bhattacharyya \& Haddara, 2006).

There are two common maneuvers that are used for estimation and validation of the nonlinear hydrodynamic derivatives: the circle test and the zig-zag maneuver. The circle test is performed at constant thrust, and a steady turn is established through use of the rudder, typically for at least $540^{\circ}$ of a circle. The zig-zag maneuver is exactly how it sounds, a zig-zag where the heading is changed by a prescribed amount through a constant rudder deflection. When the new heading is obtained, the rudder is deflected the same amount in the other direction until the boat reaches the same deviation from the original heading in the other direction. The change from original heading and rudder deflection angle to use are specified in the name, so a 20-20 zig-zag maneuver means $20^{\circ}$ left and right of the original heading accomplished through $20^{\circ}$ of rudder deflection. In one case these standard maneuvers have been used to identify the hydrodynamic parameters of a ship including the roll DOF (Wang, 2012). This was achieved with the estimation-before-modeling technique which is a two-step process beginning with an extended 
Kalman filter followed by a modified Bryson-Frazier smoother. The ship used for this study was intended to represent fast container ships or naval vessels which exhibit more significant rolling motion during turns than traditional ships.

The circle test cannot be performed on a sailing yacht because a sailboat cannot sail in a perfect circle and also cannot maintain constant thrust during such a maneuver. The zig-zag maneuver could prove useful, but the aerodynamic forces and moments would have to be known for the yacht at each point in the maneuver. It would also be impossible to do under constant thrust. These maneuvers are not meant to induce large changes in heel, something that needs to be quantified in the hydrodynamic derivatives of a sailing yacht. For these reasons hydrodynamic derivatives for sailing yacht hulls are usually measured in a towing tank.

This chapter has presented a thorough review of the state of the art in the physics based modeling of sailing yachts, current autonomous sailboats, and system identification methods which have been applied to powered surface ships. It has been shown that there have been no investigations into sailing yacht dynamics specifically with control system design in mind, and that absence is what this dissertation is intended to address. 


\section{Non-linear Dynamic Models of a Sailing Yacht}

\subsection{Non-linear Dynamic Systems Theory}

For sailing yachts, it is possible to describe the dynamic yacht system with a set of physicsbased differential equations. For vehicles, these are most often equations of motion derived from Newtonian physics. This set of differential equations represents the model, and contains the information necessary understanding and predicting the physical system behavior. State space model representations are the differential equations for the changes in the states of the system in terms of the states, inputs (controls), and a set of parameters that uniquely define the dynamic characteristics of the system. These representations are generally necessary for parameter estimation. A general non-linear state space model form is given by (Jategaonkar, 2006):

$$
\begin{aligned}
& \dot{\vec{x}}(t)=f[\vec{x}(t), \vec{u}(t), \vec{\beta}]+F \vec{w}(t), \quad \vec{x}\left(t_{0}\right)=\vec{x}_{0} \\
& \vec{y}(t)=g[\vec{x}(t), \vec{u}(t), \vec{\beta}]
\end{aligned}
$$

Where $\vec{x}$ is the vector of states, $\vec{u}$ is the vector of inputs or controls, $\vec{\beta}$ is the vector of system parameters, and $\vec{y}$ is the system output. The matrix $F$ and vector $\vec{w}$ represent the generally un-measurable additive process noise. The functions $f$ and $g$ are usually non-linear realvalued functions, and $\vec{x}_{0}$ is the initial condition of the system.

For a sailing yacht, the states are a set of variables that adequately define the yacht's motion for the purpose of predicting future motion. To describe the boat dynamics in the body coordinate system, the states should include boat speed $\left(V_{B}\right)$, heel angle $(\phi)$, and true wind speed $\left(V_{T}\right)$. Herein lies the trouble in using state space form to represent sailing yacht dynamics. One would traditionally think of the wind as an external input to the system, however, it is uncontrollable in nature, and thus cannot be considered a control input to the yacht dynamic system. If wind is included in the model as a state, a differential equation to describe how it changes with time is necessary, but changes in the true wind over time are not predictable. However, wind must be included somewhere in the model because a sailing yacht is extremely sensitive to changes in the true wind. For non-linear estimation, a solution to this dilemma is to treat the wind simply as a measured input to the discrete system. Measurements of apparent wind speed $\left(V_{A}\right)$ and direction $\left(\beta_{A}\right)$ are made during testing, thus, their time histories are known and 
may be sent into the system during estimation. This does not solve the problem for control design, but it is an adequate solution for parameter estimation.

The vector of system parameters, $\vec{\beta}$, is what is identified with system identification. For the sailing yacht this means estimating both aerodynamic and hydrodynamic parameters. Aerodynamic parameters such as the lift curve slope of the sail must be defined at varying angles

of attack, translated into yachting terms that means varying true wind angles. In order for the sails to be considered as controls, at each of the varying true wind angles, the effects of changing the sail away from the optimal steady state condition must also be characterized. This is described in Section 3.4.3 where a new aerodynamic model has been proposed and validated with the full scale data. The hydrodynamic model, depending on how it is defined, requires estimation of parameters at a minimum of several boat speeds from zero up to the hull speed of the boat. For a better hydrodynamic model, estimation of parameters at varying roll angles is also necessary as the hydrodynamics of a sailing yacht hull change significantly as it heels from zero to as much as 45 degrees. In short, this all means that in order to fully characterize or estimate the non-linear model of a sailing yacht using system identification techniques, motion data must be obtained covering the entire operating range of the boat. This is a substantial requirement, and certainly has influenced many decisions made throughout this project.

\subsubsection{Coordinate Systems}

Before entering into a detailed discussion of the equations of motion of a yacht, first a reference system must be defined. For a more detailed description of these frames and their transformations, see (Stengel, 2004). It is useful to define a body fixed coordinate system whose origin lies at the center of mass, $O=\left\{x_{b}, y_{b}, z_{b}\right\}$, of the vehicle as shown in Figure 1. In the body fixed frame of a yacht, the $\mathrm{x}$ direction points to the bow of the boat, the $\mathrm{y}$ direction to port, and the $\mathrm{z}$ direction down into the water. In this frame, linear translational motions are described by a vector $\mathbf{v}_{B}=\{U, V, W\}^{T}$ and angular motions are described by a vector $\boldsymbol{\omega}_{B}=\{P, Q, R\}^{T}$ as shown in Table 1. 
Table 1: List of Velocities in Body Fixed Coordinate Frame

\begin{tabular}{|cccc|}
\hline Linear Velocities & Names & Angular Rates & Names \\
\hline$U$ & Surge & $P$ & Roll \\
$V$ & Sway & $Q$ & Pitch \\
$W$ & Heave & $\boldsymbol{R}$ & Yaw \\
\hline
\end{tabular}

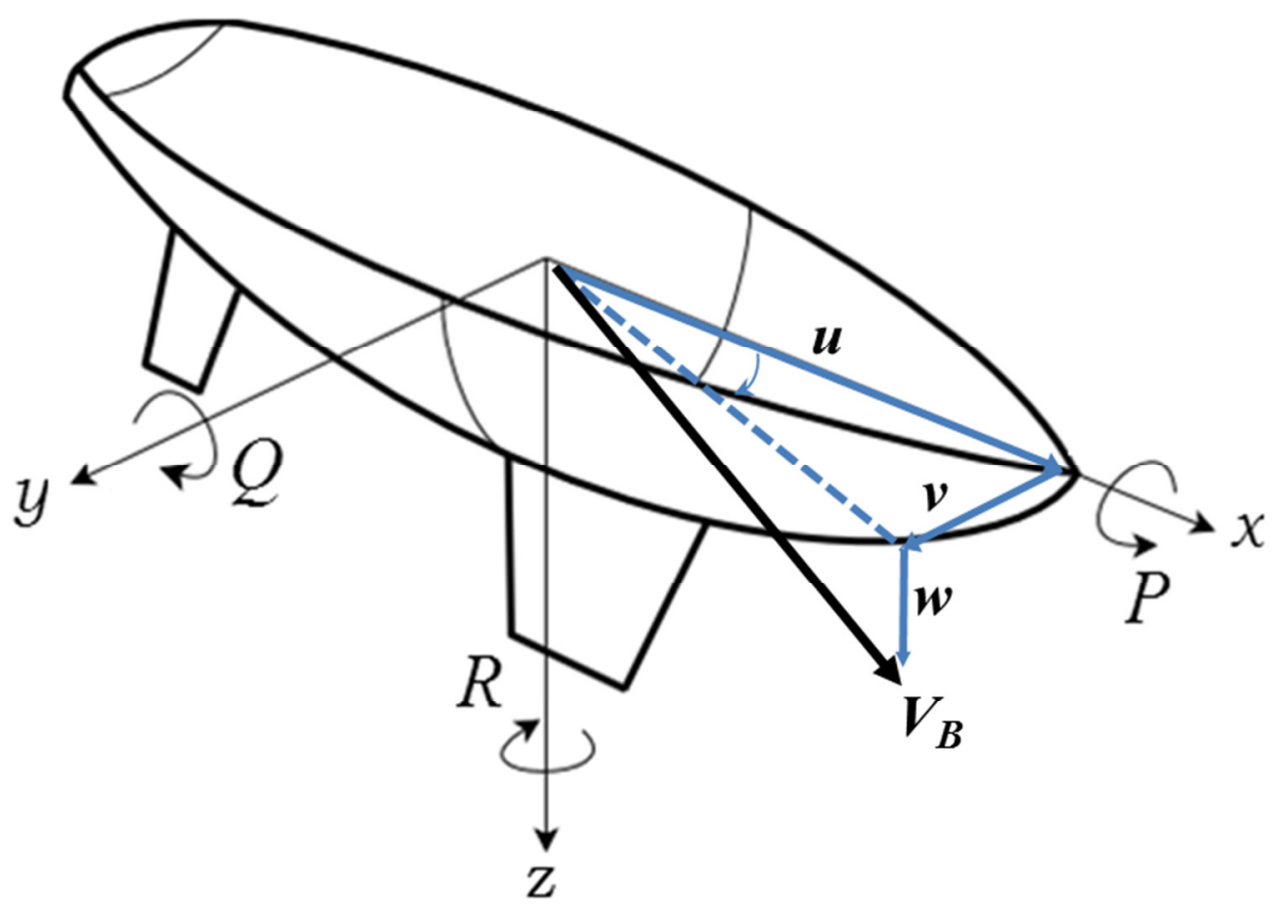

Figure 1: Body Coordinate System with Linear Velocities

In the body fixed frame, there is no information about the attitude of the boat. In other words, it does not define the position of the boat relative to the water it is sailing upon. The local inertial coordinate frame contains such information and is defined relative to the body fixed coordinate systems through the Euler angles, $\Theta=\{\phi, \theta, \psi\}^{T}$ representing roll, pitch and yaw. 


\subsection{Four DOF Non-Linear Dynamic Model}

The physics based model that will be used throughout this work is based on the rigid body equations of motion expressed in the body coordinate system. In order to simplify the model and make it suitable for preliminary system identification, the heave and pitch degrees of freedom have been removed from the model. The majority of forces that cause heave and pitch motion on a monohull sailing yacht are the uncontrollable forces generated by waves, and for this study it is appropriate to exclude them from the model. It is extremely common for surface vessels to use only a 3DOF model for maneuvering, where roll is excluded as well (Fossen, 2011). The roll degree of freedom must be included for the sailing yacht because it experiences large changes in heel as part of its normal range of operation.

For a vessel symmetric about the $x z$ plane, the four degree of freedom rigid body equations of motion expressed in a body coordinate system fixed to the center of gravity can be fully expressed as (Fossen, 2011; Stengel, 2004):

$$
\begin{gathered}
\dot{U}=X / m+V R \\
\dot{V}=Y / m-U R \\
\dot{P}=\left(I_{z z} K+I_{x z} N\right)\left(I_{x x} I_{z z}-I_{x z}^{2}\right)^{-1} \\
\dot{R}=\left(I_{x x} N+I_{x z} K\right)\left(I_{x x} I_{z z}-I_{x z}^{2}\right)^{-1}
\end{gathered}
$$

Where $U$ and $V$ are the velocities along the surge and sway axes, and $P$ and $R$ are the angular velocities about the roll and yaw axes, all in the body fixed coordinate system. $X$ and $Y$ represent the total external forces acting along the surge and sway axes; $K$, and $N$ represent the total external moments applied around the roll and yaw axes. For a sailing yacht, the forces and moments are generated by the sails, hull, keel, and rudder. The effects of added mass and buoyancy/righting moment must also be included in the model, and in this work have been included as part of the hydrodynamic force and moment model.

A good starting point for a force model appropriate for system identification is presented by Masuyama (Masuyama, Fukasawa, \& Sasagawa, 1995) with sail, hull/keel, and added mass force and moment coefficients. According to this model, the external forces and moments can be written as: 


$$
\begin{gathered}
X=X_{0}(U)+X_{H y d r o}+X_{R u d}+X_{S} \\
Y=Y_{H y d r o}+Y_{R u d}+Y_{S} \\
K=K_{\text {Hydro }}+K_{R u d}+K_{S}-m g \overline{G M} \sin \phi \\
N=N_{H y d r o}+N_{R u d}+N_{S}
\end{gathered}
$$

where $X_{0}$ represents total upright hull drag which is a function of forward velocity, the subscript Hydro denotes hydrodynamic forces and moments, Rud denotes forces and moments generated by the rudder, and $S$ denotes aerodynamic forces and moments generated by the sail plan. $\mathrm{g}$ is acceleration due to gravity, and $\overline{G M}$ is the yacht's metacentric height. The equations defining hydrodynamic, rudder, and sail forces and moments will be expanded upon in the following sections.

\subsection{Modeling Hydrodynamic Forces and Moments}

The hydrodynamic forces developed on a hull moving through the water involve many complex interactions for which no numerical solution has yet been devised. Thus, it is useful to break down these forces into individual components which when combined provide an overall picture of the hydrodynamics of the hull. This breakdown is shown in Figure 2. In the hydrodynamics community, it is common to use the terms resistance and drag interchangeably to describe the forces that resist the forward motion of the yacht, and will be used so in the following sections.

The total hydrodynamic force can be broken into two main categories, the forces generated by the canoe body of the hull, and the forces generated by the appendages (the keel and rudder). Keels and rudders are symmetric airfoils that will only generate forces other than skin friction drag when the yacht is sailing with leeway, and when the rudder is deflected away from centerline. Any time the yacht is sailing with a heel angle, it sails with leeway, so the induced drag of the appendages is generally separated from the lift and drag they produced. This is not the standard approach in aerospace which includes the induced drag as part of the overall drag generated by the wing. This unusual approach is taken for sailing yachts because the effects of resistance for a yacht sailing upright with no leeway have been well defined empirically, so it makes sense to consider the added effects due to heel separately. Heel not only causes induced 


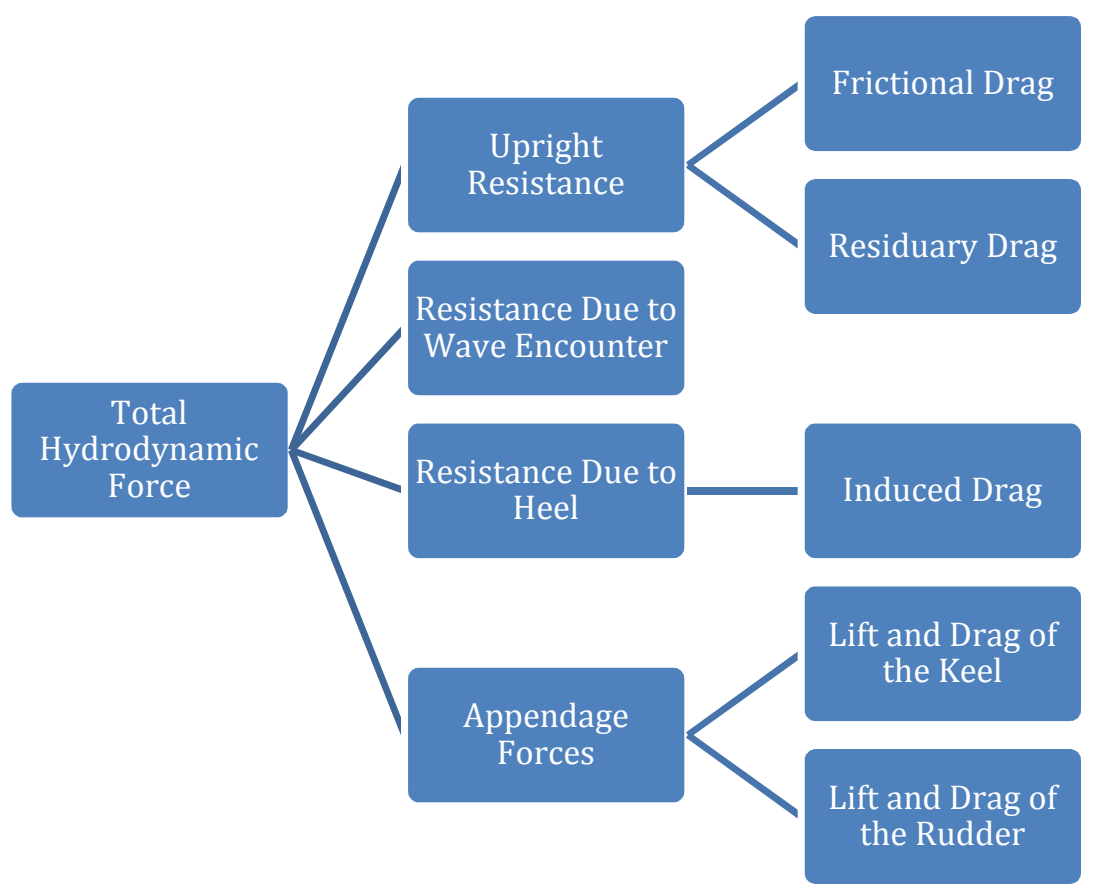

Figure 2: Breakdown of total resistance forces on a sailing yacht hull.

drag from the appendages, but it also changes the wetted surface area and shape of the hull. Thus, it is often included in a hydrodynamic model as a separate component of the overall drag.

There are two approaches that are used in the literature to model the hydrodynamics of a sailing yacht. One is similar to the theoretical models used for ships and makes use of nondimensional hydrodynamic derivatives which are characterized in a towing tank upright and at different heel angles. The second is the widely used empirical model based on the DSYHS. Both methods have been used throughout this project to achieve different ends, and will be discussed in the following two sections.

\subsubsection{Modeling with Hydrodynamic Derivatives}

Operating in the highly viscous medium of water introduces some interesting phenomena that have been studied in great length by the naval architecture and hydrodynamics community for the purposes of ship maneuvering. There are several non-linear maneuvering models which are used to describe both the steady and dynamic forces and moments that act on a hull maneuvering in water. Many of these models are formulated only in 3DOF systems for ship maneuvering. Fossen (Fossen, 2011) offers three models from the literature for 4DOF ship 
maneuvering models which include roll: the nonlinear model of Son and Nomoto (Son \& Nomoto, 1982), the nonlinear model of Blanke and Christensen (Blanke \& Christensen, 1993), and the nonlinear model based on low aspect ratio wing theory (Ross, 2008). The first of these models was adopted by Masuyama and simplified for application to sailing yacht canoe body and keels (Masuyama et al., 1995):

$$
\begin{gathered}
X_{H}=\left(X_{V V}^{\prime} V^{\prime 2}+X_{\phi \phi}^{\prime} \phi^{2}+X_{V R}^{\prime} V R+X_{V V V V}^{\prime} V^{\prime 4}\right)\left(1 / 2 \rho_{w} V_{B}^{2} L_{W L} D_{K}\right) \\
Y_{H}=\left(Y_{V}^{\prime} V^{\prime}+Y_{\phi}^{\prime} \phi+Y_{V \phi \phi}^{\prime} V^{\prime} \phi^{2}+Y_{V V \phi}^{\prime} V^{\prime 2} \phi+Y_{V V V}^{\prime} V^{\prime 3}\right)\left(1 / 2 \rho_{w} V_{B}{ }^{2} L_{W L} D_{K}\right)+Y_{P} P+Y_{R} R \\
K_{H}=\left(K_{V}^{\prime} V^{\prime}+K_{\phi}^{\prime} \phi+K_{V \phi \phi}^{\prime} V^{\prime} \phi^{2}+K_{V V \phi}^{\prime} V^{\prime 2} \phi+K_{V V V}^{\prime} V^{\prime 3}\right)\left(1 / 2 \rho_{w} V_{B}{ }^{2} L_{W L} D_{K}{ }^{2}\right)+K_{P} P \\
N_{H}=\left(N_{V}^{\prime} V^{\prime}+N_{\phi}^{\prime} \phi+N_{V \phi \phi}^{\prime} V^{\prime} \phi^{2}+N_{V V \phi}^{\prime} V^{\prime 2} \phi+N_{V V V}^{\prime} V^{\prime 3}\right)\left(1 / 2 \rho_{w} V_{B}{ }^{2} L_{W L}{ }^{2} D_{K}\right)+N_{R} R
\end{gathered}
$$

Where:

$$
\begin{gathered}
V^{\prime}=-\sin \gamma=-\sin \left(\tan ^{-1}(V / U)\right) \\
\gamma=\tan ^{-1}(V / U) \\
V_{B}^{2}=U^{2}+V^{2}
\end{gathered}
$$

The dynamic terms in Eqs. (3.10) - (3.13) are $X_{V \dot{\mathrm{R}}}, Y_{\mathrm{P}}, Y_{\mathrm{R}}, K_{\mathrm{P}}$, and $N_{\mathrm{R}}$. The steady state terms are the rest of the derivatives, and as can be seen these are based upon leeway and heel, thus following the force breakdown in Figure 2.These hydrodynamic derivatives are defined in the following manner:

$$
X_{V V}^{\prime}=\frac{\partial^{2} X}{\partial V^{\prime 2}} \quad X_{V R}^{\prime}=\frac{\partial^{2} X}{\partial V \partial R} \quad Y_{P}=\frac{\partial Y}{\partial P} \quad \cdots
$$

A note here is that in modifying the ship equations for a sailing yacht with a keel, in this particular model the lift and drag generated by the hull and keel have been lumped together and are thus accounted for in the hydrodynamic derivatives. These are clearly highly nonlinear expressions, yet it is common to omit some of the higher order terms as their contribution to overall forces and moments are small. 


\subsubsection{Modeling with Delft Systematic Yacht Hull Series (DSYHS)}

The DSYHS is a way to model the drag forces of a sailing yacht hull which is based on summing upright resistance, added resistance due to heel, and added forces from leeway. The DSYHS is based on extensive towing tank tests of 70 different sailing yacht hull forms (Keuning $\&$ Katgert, 2008). These hull forms are systematically varied in geometric parameters based on 6 different 'parent' hull forms. The results are used to compose polynomial expressions fit regressively to the data produced by the towing tank tests. The polynomial expressions are composed of a set of the coefficients obtained from the regression analysis multiplied by geometrical relationships of the hull. The set of these expressions and the associated geometrical parameters for the Precision 23 may be found in Appendix B. This series only applies to hull and keel forms, and adds the residuary and frictional drag of the keel and rudder, but does not account for lift and drag of the rudder, so if this model is used to calculate total hydrodynamic forces and moments, wing theory may be applied to calculate forces from the rudder.

\subsubsection{Rudder Forces and Moments}

There are two models for rudder forces and moments that are used in this work. The first is taken directly out of a previous model (Masayuma \& Fukasawa, 2011) and will be used in conjunction with the non-linear simulations presented in Section 3.5. This model is given in Eqs. (3.18) - (3.21):

$$
\begin{gathered}
X_{R u d}=C_{X \delta_{R}} \sin \alpha_{R} \sin \delta_{R} \cdot 1 / 2 \rho_{w} V_{B}^{2} L_{W L} D_{K} \\
Y_{R u d}=C_{Y \delta_{R}} \sin \alpha_{R} \cos \delta_{R} \cos \phi \cdot 1 / 2 \rho_{w} V_{B}^{2} L_{W L} D_{K} \\
K_{R u d}=C_{K \delta_{R}} \sin \alpha_{R} \sin \delta_{R} \cdot 1 / 2 \rho_{w} V_{B}^{2} L_{W L} D_{K}^{2} \\
N_{R u d}=C_{N \delta_{R}} \sin \alpha_{R} \cos \delta_{R} \cos \phi \cdot 1 / 2 \rho_{w} V_{B}^{2} L_{W L}^{2} D_{K}
\end{gathered}
$$

Where $C_{X \delta R}, C_{Y \delta R}, C_{K \delta R}$, and $C_{N \delta R}$ are non-dimensional coefficients, $\delta_{R}$ is the physical rudder deflection from centerline, and $\alpha_{R}$ is the effective angle of attack on the rudder as defined by Eq. (3.22):

$$
\alpha_{R}=\delta_{R}-\varepsilon_{\gamma} \gamma-\tan ^{1}\left(\frac{x_{R} R}{U}\right)
$$




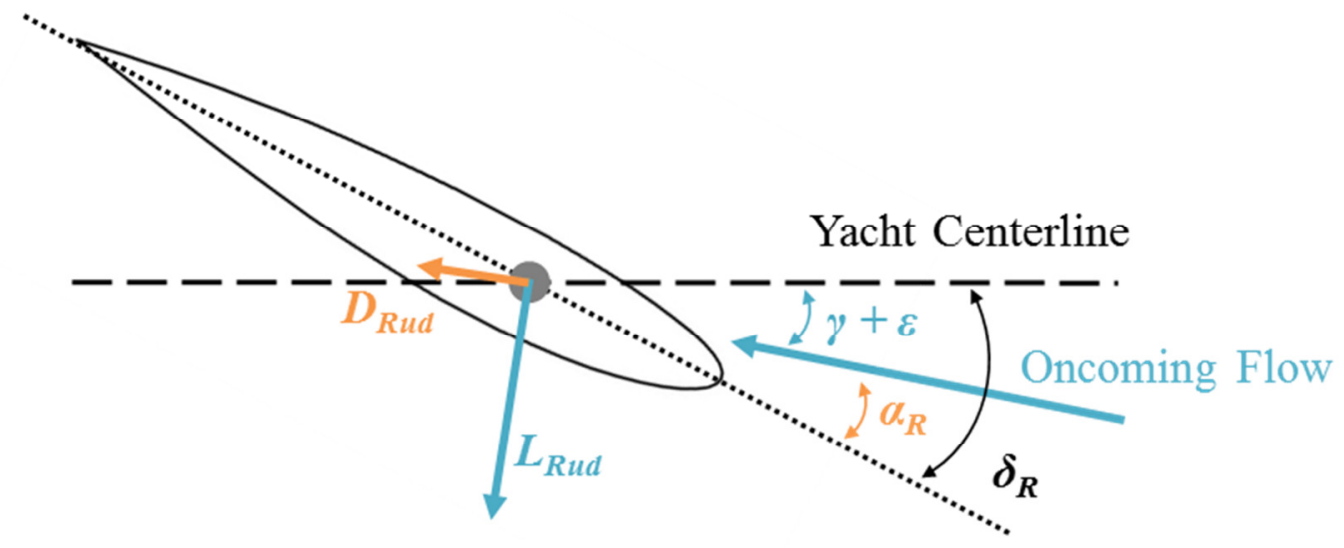

Figure 3: Definition of rudder deflection. All the angles shown are positive.

$$
\varepsilon=\frac{d \varepsilon}{d \gamma} \cdot \gamma=\varepsilon_{\gamma} \gamma
$$

Where $\gamma$ is the leeway angle the yacht is sailing and $\varepsilon$ is the angle of inflow from the downwash generated by the keel, and $x_{R}$ is the longitudinal distance of the quarter-chord point of the rudder to the c.g. of the boat.

The second model has been used in conjunction with the DSYHS to generate estimates of the rudder forces on the Precision 23. It uses Hembold's modification to lifting line theory for low aspect ratio wings (Anderson, 2007) to generate lift and drag forces on the rudder, which are then transformed into the body coordinates. The lift coefficient of the rudder, $C_{L R}$, treated as a wing may be written as:

$$
\begin{gathered}
C_{L_{R}}=\frac{c_{l_{R}}}{\sqrt{1+\left(c_{l_{R}} / \pi A R_{R}\right)^{2}+c_{l_{R}} / \pi A R_{R}}} \\
c_{l_{R}}=f\left(\alpha_{R}\right)
\end{gathered}
$$

Where $c_{l R}$ is the lift curve slope of the rudder's airfoil section and is a function of the rudder's effective angle of attack, and $A R_{R}$ is the aspect ratio of the rudder. The effective angle of attack on the rudder is defined as:

$$
\alpha_{R}=\delta_{R}-(\gamma+\varepsilon)
$$

The corresponding total drag coefficient, $C_{D R}$, of the rudder is given by: 


$$
C_{D_{R}}=c_{d_{R}}+\frac{C_{L_{R}}^{2}}{\pi e A R_{R}}
$$

Where $c_{d R}$ is the profile drag coefficient of the rudder, and $e$ is Oswald's efficiency factor. The lift force acts perpendicular to the oncoming flow, and the drag force acts parallel to the oncoming flow, as pictured in Figure 3 which also defines the sign convention used for the rudder model because it shows all positive angles. The total lift and drag forces on the rudder may be written as:

$$
\begin{gathered}
L_{\text {Rud }}=C_{L_{R}} 1 / 2 \rho_{w} V_{B}^{2} S_{R u d} \\
D_{\text {Rud }}=C_{D_{R}} 1 / 2 \rho_{w} V_{B}^{2} S_{R u d}
\end{gathered}
$$

Where $S_{\text {rud }}$ is the wetted surface area of the rudder. To transform these into the body axis frame:

$$
\begin{gathered}
X_{\text {Rud }}=-\left(L_{\text {Rud }} \sin (\gamma+\varepsilon)+D_{\text {Rud }} \cos ((\gamma+\varepsilon))\right) \\
Y_{\text {Rud }}=L_{\text {Rud }} \cos (\gamma+\varepsilon)-D_{\text {Rud }} \sin (\gamma+\varepsilon)
\end{gathered}
$$

The moments are then defined by the longitudinal and vertical distances of the rudder's center of effort, $x_{R}$ and $z_{R}$, from the center of gravity:

$$
\begin{aligned}
& K_{R u d}=Y_{R u d} z_{R} \\
& N_{R u d}=Y_{R u d} x_{R}
\end{aligned}
$$

\subsection{Modeling Aerodynamic Forces and Moments}

The source of thrust for a sailing yacht is the wind. The true wind and its direction with respect to the yacht completely govern the speed and direction the boat is able to sail. In practice, the true wind and its direction cannot be measured, but the apparent wind speed and direction can be measured by a mast head anemometer. The apparent wind is created by the fact that not only is the yacht in the presence of the true wind, it is also moving with a velocity, $V_{B}$, through that wind, thus creating the apparent wind. Apparent wind velocity, $V_{A}$, is defined as the vector combination of the boat velocity, $V_{B}$, and the true wind velocity, $V_{T}$, as shown in Figure 4 . Also defined in this figure are the mainsail angle, $\delta_{b}$, and leeway angle, $\gamma$. Note that leeway is defined in Eq. (3.15), and in Figure 4 it is negative, and the apparent wind angle $\beta_{A}$ is shown as positive. 


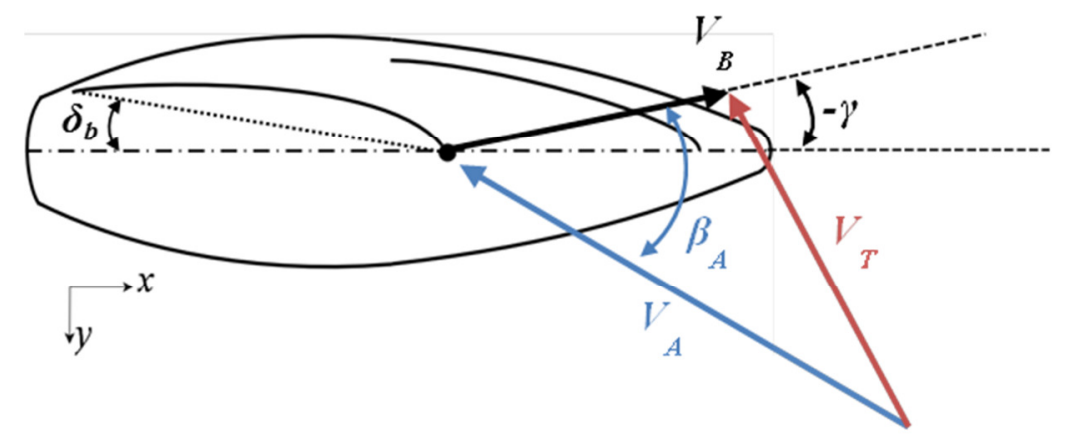

Figure 4: The Wind Triangle

\subsubsection{Traditional Methods}

Aerodynamic forces generated on a sailing yacht are very complicated to model due to the nature of flexible sails. Generally, the lift and drag forces generated by the sail plan are modeled using non-dimensional force coefficients. The sail coefficients are not only dependent upon apparent wind angle, $\beta_{A}$, but also highly dependent upon heel(roll) angle, $\phi$. An accepted solution to the dependence of aerodynamic coefficients on heel is to use effective angle theory (Heikki Hansen, 2006; Kerwin, 1978) to define an effective wind angle, $\beta_{\text {eff, }}$ and velocity, $V_{\text {eff, in }}$ the heeled plane, and use those variables rather than $\beta_{A}$ and $V_{A}$ to define the coefficients. For our application of 4 DOF excluding heave and pitch, the $\beta_{\text {eff }}$ and $V_{\text {eff }}$ are defined as follows (Heikki Hansen, 2006):

$$
\begin{gathered}
\beta_{e f f}=\tan ^{-1}\left(\tan \beta_{A} \cos \phi\right) \\
V_{\text {eff }}=V_{A} \sqrt{1-\sin ^{2} \beta_{A} \sin ^{2} \phi}
\end{gathered}
$$

Typical curves representing a complete set of traditionally reported optimal sail coefficients can be seen in Figure 5. These numbers are based on lift and drag coefficients that have been rotated into the body axis frame. For a more detailed description of this process see the Appendix of (Masayuma \& Fukasawa, 2011). 


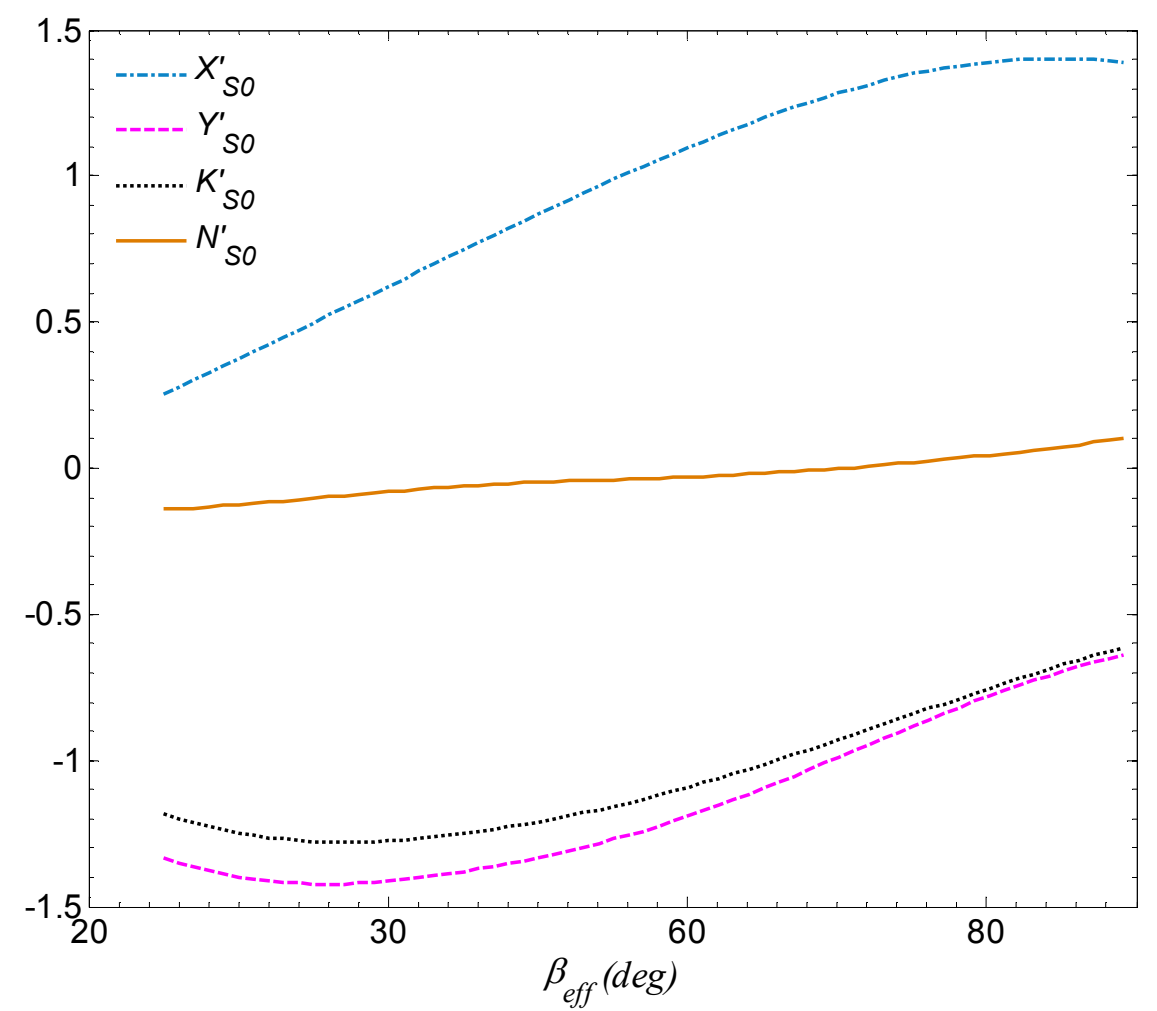

Figure 5: Typical sailing yacht force and moment coefficients shown as functions of effective wind angle

Aerodynamic data for traditional yacht models found in VPP's is reported using the 'optimum' sail angle which produces the best "velocity made good" for the sailing yacht for either an apparent or true wind angle (Ciortan \& Guedes Soares, 2007; Heikki Hansen, 2006; Masuyama et al., 2009). Different methods have been used to describe how the sails can be modified away from their fully powered up optimal condition. The most common is the early solution that modifies the coefficients using the parameters of reef and flat (Kerwin, 1978) to represent decreases from the optimal sail coefficients that occur when sailing in real-life conditions. An alternate depowering model (Heikki Hansen, 2006) uses a single parameter, power, which is based on the ratio between the real heeling moment coefficient and the optimum, to modify aerodynamic coefficients. Both of these models aim to capture the effect of utilizing the many secondary controls available on a sailing yacht such as reefing points, outhauls, boom vangs, etc that are used to depower and trim the sails. They are utilized in the equations simply as factors that may be adjusted if the yacht is not balanced, they do no relate any physical information about the state of the sails on the yacht itself. 
Sail shape capture during full-scale tests currently the most utilized means of validation of aerodynamic models. This is done with a set of cameras on the boat being analyzed or a chase boat, the cameras take images of sails with large chord stripes or dots painted on the sails. Sail shape capture on full-scale yachts coupled with CFD analysis has been used to evaluate a yachts upwind performance (Masuyama et al., 2009). Investigations into the shape of downwind sails have been made for the purpose of giving sailors concrete trimming guidelines for spinnakers (Graf, Olaf Muller). There is a commercial product developed by the Yacht Research Unit at the University of Auckland called VSPARS ${ }^{\complement}$ which is a complete camera/software package which allows real time analysis of sail shape (Le Pelley \& Modral, 2008). This product is mostly utilized by teams competing in long distance races because it allows them to periodically evaluate sail trim during long legs.

It is clear that sail shape is a driving factor in sailing yacht performance, but changes in sail shape are made through simultaneous adjustment of numerous controls. The sail shape capture systems are all focused on attaining the optimum sail shape for given conditions, but it is still up to the human crew to decide how best to modify controls to achieve those shapes. All existing aerodynamic performance models assume the sails have been optimally trimmed for conditions.

\subsubsection{New Aerodynamic Force Model Including Sail Deflection}

The drawback of current aerodynamic models for sailing yachts is the assumption that sails are in optimal trim when reporting coefficients. The goal of an autonomous sailing yacht will likely be very different than that of a racing boat, tenths of a knot gains in speed will not be as important as maintaining a constant course, safety, and minimizing wear on the yacht itself. It would be extremely useful for an autonomous control system for a sailing yacht to exploit the sails as real control inputs to the dynamic system. At the simplest level, that means including sail deflections in the physics based dynamic model of sailing yachts, something non-existent in current models.

The goal of control is to define the simplest model that effectively captures the vehicle dynamics, and existing models do not relate the sail coefficients to a single, simple control. The most straightforward way to depower a sail plan is to let the main sail out (decrease its deflection angle, $\delta_{B}$ ) to decrease heeling force. When this is insufficient, the entire sail area should be reduced (reefing). For control applications, it is the effect of the whole sail plan that is important, 
meaning the combined effect of the main sail and jib, genoa, or spinnaker. The new model here is inspired by those used in aerospace control applications where total airplane aerodynamic coefficients are modified by linear curves. For example, the typical breakdown for the total lift coefficient of an aircraft is(Roskam, 2007):

$$
C_{L}=C_{L_{0}}+C_{L_{\alpha}} \alpha+C_{L_{i h}} i_{h}+C_{L_{\delta e}} \delta_{e}
$$

Where, $\alpha$ is angle of attack, $i_{h}$ is the incidence angle of the horizontal tail, and $\delta_{e}$ is the elevator deflection angle. $C_{L 0}$ is the lift coefficient when $\alpha=i_{h}=\delta_{e}=0$, and the rest are linear slopes of the change in total lift with changes in $\alpha, i_{h}$ and $\delta_{e}$. This section presents a new model similar to that in Eq. (3.36), and describes the effect of the entire sail plan, then investigates its ability to model sailing yacht dynamics through simulation and analysis of data collected aboard a full-scale yacht.

Aerodynamic sail forces and moments in the new model are described in 4DOF by a vector of driving force, $X_{S}$, side force, $Y_{S}$, rolling moment, $K_{S}$, and yawing moment, $N_{S}$ :

$$
f_{\text {aero }}=\left[\begin{array}{llll}
X_{S} & Y_{S} & K_{S} & N_{S}
\end{array}\right]^{T}
$$

The new model is a modification of a previous model(Masuyuma, Nakamura, Hisayoshi, \& Takagi, 1993) that describes the aerodynamic forces in the body coordinate system rather than in terms of lift and drag coefficients:

$$
f_{\text {aero }}=1 / 2 \rho_{a} V_{\text {eff }}{ }^{2} S_{A} *\left[\begin{array}{llll}
X_{S}^{\prime} & Y_{S}^{\prime} & \sqrt{S_{A}} K_{S}^{\prime} & \sqrt{S_{A}} N_{S}^{\prime}
\end{array}\right]^{T}
$$

The non-dimensional coefficients, $X_{S}^{\prime}, Y_{S}^{\prime}, K_{S}^{\prime}$, and $N_{S}^{\prime}$, represent the total aerodynamic force and moment coefficients. $S_{A}$ is the reference sail area, and $\rho_{a}$ is the air density. The reference sail area is calculated by the traditional IMS racing formulation:

$$
S_{A}=\frac{S_{\operatorname{main}}}{1.16}+\frac{I J}{2}
$$




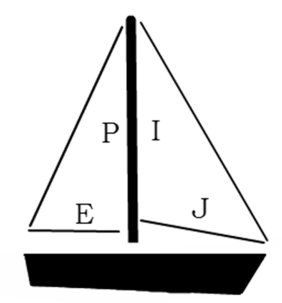

\section{Figure 6: IMS Sail Dimensions}

Where $S_{\text {main }}$ is the surface area of the mainsail, and $I$ and $J$ are the principal definitions of the foresail as shown in Figure 6 .

The total sail angle $\delta_{b}$ is measured w.r.t. the centerline of the yacht (See Figure 4) and may be expressed as the sum of the optimal sail angle plus the deviation of the angle from the optimum, $\Delta \delta_{b 0}$ :

$$
\delta_{b}=\delta_{b_{0}}+\Delta \delta_{b_{0}}
$$

The new aerodynamic model proposed includes an additional coefficient representing the change in force or moment coefficient w.r.t. $\Delta \delta_{b 0}$ :

$$
X_{S_{s b}}^{\prime}=\frac{d X_{S}^{\prime}}{d \delta_{b}}
$$

Therefore, the new expression for the total sail force coefficient (in surge) is written as:

$$
X_{S}^{\prime}=\left(X_{S_{0}}^{\prime}+X_{S_{b b}}^{\prime} \Delta \delta_{b_{0}}\right)
$$

The term $X_{S_{0}}^{\prime}$ represents the sail coefficient at the optimal sail deflection angle, and thus also represents the curves in Figure 5. This new model, which has a similar structure to Eq. (3.36), has the advantage of directly relating a control variable to the resulting forces and moments, which has not been common in past models. It would be ideal if $X_{S \delta b}^{\prime}$ was constant for all $\beta_{\text {eff; }}$ but this is likely not the case, and it too will be dependent, as $X_{S_{0}}^{\prime}$ is, on $\beta_{\text {eff. }}$ 


\subsubsection{Validation of New Model with Full-scale Data}

The new aerodynamic model has been verified using a set of full-scale data taken with the Precision 23 data acquisition system described in Chapter 5. The data analyzed and presented here all represents data collected while sailing on port tack at varying apparent wind angles. The optimal sail deflections are not known for the Precision 23, so they are estimated from the data. A total of seven data segments representing a total of about 10 minutes of near steady state trimmed sailing are individually averaged, resulting in seven pairs of $\left(\beta_{\text {eff }}, \delta_{b 0}\right)$, shown Figure 7. The standard deviations of these averages are very small, so the points are an accurate representation of the measurement for that particular time segment. A linear curve is fitted to these average points with $R^{2}$ of 0.67 , resulting in Eq.(3.43), which is used in all subsequent analysis to calculate $\delta_{b 0}$ from measured $\beta_{\text {eff. }}$.

$$
\delta_{b_{0}}=0.58 \beta_{e f f}-20
$$

Twelve segments of data are selected, each representing about 40 seconds of sailing data, with each segment containing a change in sail deflection. The collection of these segments results in 2,062 individual data points with which to analyze the new aerodynamic model.

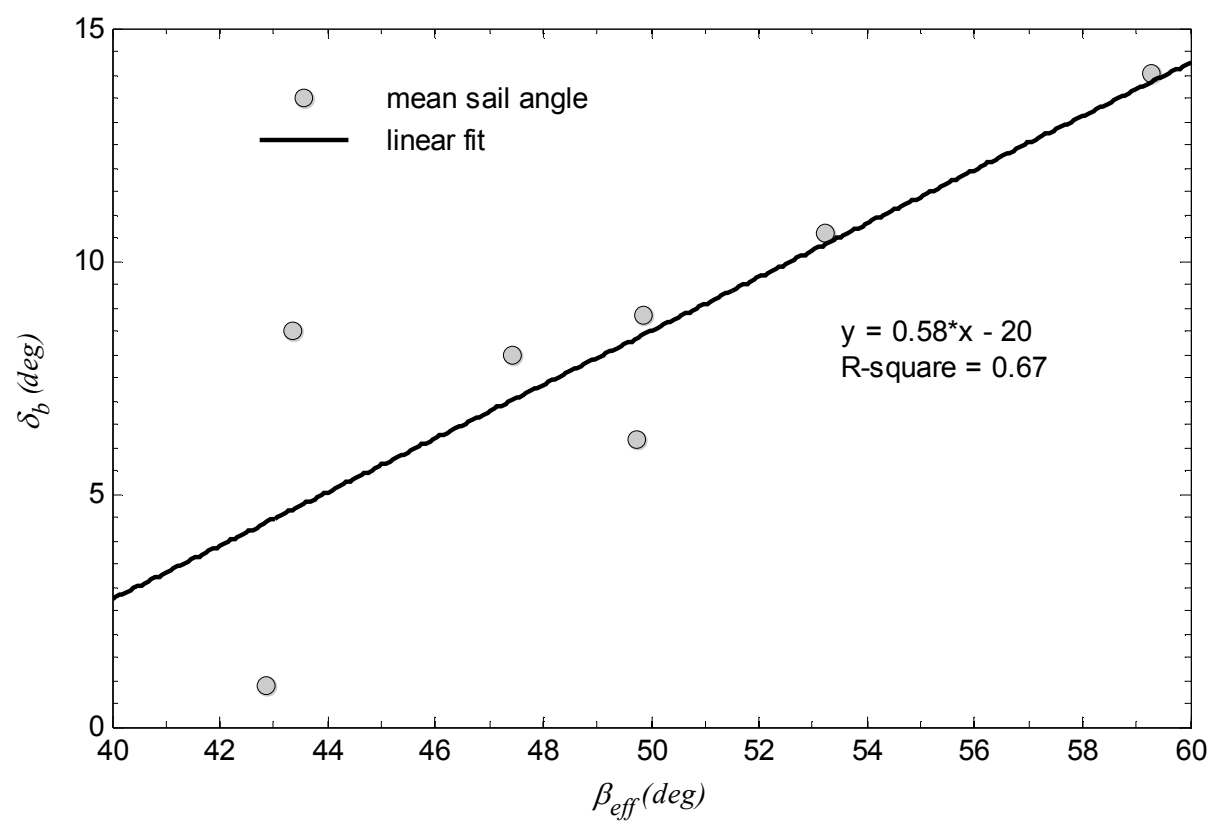

Figure 7: Linear fit from averaging 10 minutes of trimmed sailing data to estimate the optimal sail angle curve for the Precision 23 
Currently, a hydrodynamic model of the hull, keel, and rudder has not been accurately estimated for the test vehicle. An estimate of the hydrodynamic forces and moments acting upon the hull are needed in order to directly estimate aerodynamic coefficients from the data. The results of the DSYHS (Gerritsma et al., 1977; Keuning \& Katgert, 2008) are used to generate all residuary resistance/drag forces generated by the hull, both upright and due to heel. The lift and drag of the keel and rudder are estimated using Hembold's modification of lifting line theory for low-aspect-ratio wings (Anderson, 2007). A summary of hull, rudder, and keel characteristics necessary to generate these estimates is in Table 22 and Table 24, each located in Appendix B.

This hydrodynamic model is used to calculate the forces and moments generated by the hull at each of the measured data points, and the following equations are then used to solve for the total aerodynamic force and moment coefficients:

$$
\begin{aligned}
& X_{S}^{\prime}=\frac{(\dot{U}+V R)\left(m+m_{x}\right)-X_{\text {Hydro }}-X_{\text {Rud }}}{1 / 2 \rho_{a} V_{\text {eff }}^{2} S_{A}} \\
& Y_{S}^{\prime}=\frac{(\dot{V}+U R)\left(m+m_{y}\right)-Y_{\text {Hydro }}-Y_{\text {Rud }}}{1 / 2 \rho_{a} V_{\text {eff }}^{2} S_{A}} \\
& K_{S}^{\prime}=\frac{\left(I_{x x}+J_{x x}\right) \dot{P}-K_{\text {Hydro }}-K_{\text {Rud }}-m g \overline{G M} \sin \phi}{1 / 2 \rho_{a} V_{\text {eff }}^{2} S_{A} z_{C O E}} \\
& N_{S}^{\prime}=\frac{\left(I_{z z}+J_{z z}\right) \dot{R}-N_{\text {Hydro }}-N_{R u d}}{1 / 2 \rho_{a} V_{e f f}^{2} S_{A} x_{C O E}}
\end{aligned}
$$

Where $m_{x}, m_{y}, J_{x x}$, and $J_{z z}$ are added mass, $\rho_{A}$ is air density, $S_{A}$ is the reference sail area, $g$ is acceleration due to gravity, $\overline{G M}$ is the yacht's metacentric height, and $x_{C O E}$ and $z_{C O E}$ are the distances from the center of gravity (c.g.) to the center of effort of the sails. This data analysis makes no attempt to account for changes in the location of the center of effort of the sails at this time. A summary of the constant values used in Eq. (3.44) is shown in Table 23 in Appendix B. 


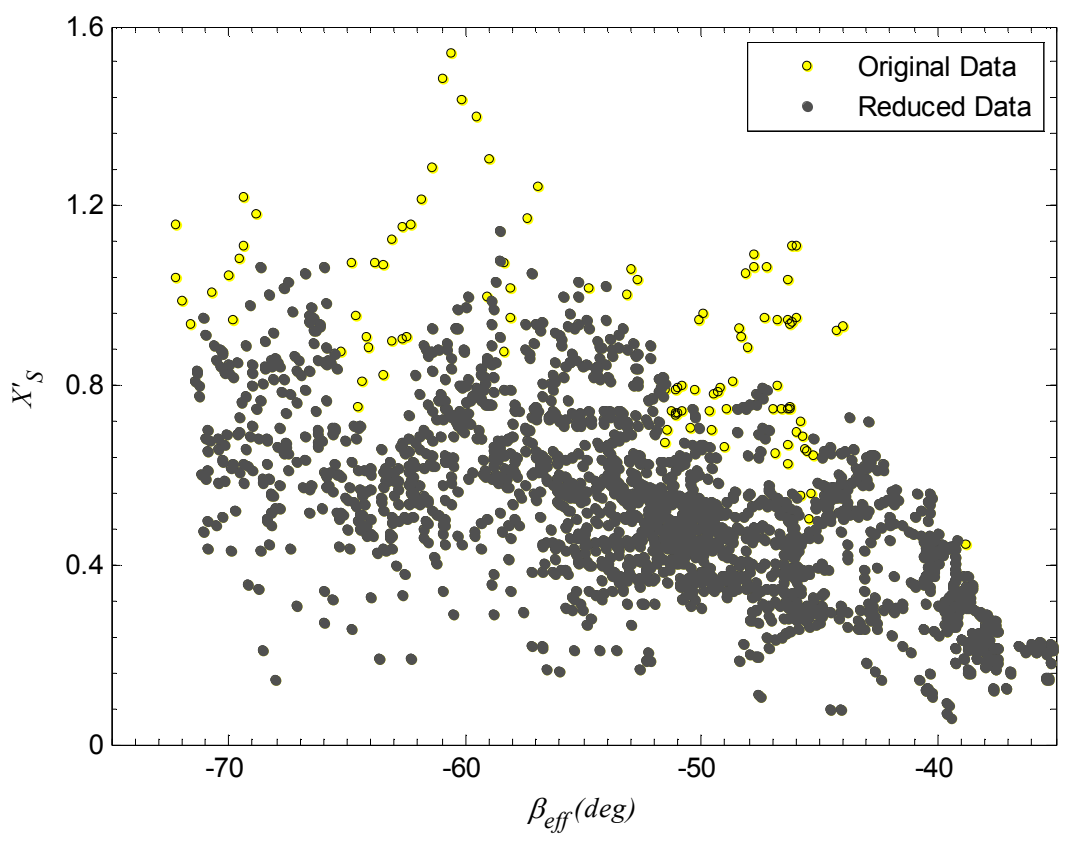

Figure 8: Original and reduced total sail coefficient data .

The total aerodynamic coefficients are thus obtained for each of the 2,062 data points, along with $\Delta \delta_{b 0}$. Only the surge degree of freedom is presented here, but the process has been repeated for sway, roll, and yaw. The first step to obtaining the unknown coefficients on the right hand side of Eq (3.42) is to look at the relationship between $X_{S}^{\prime}$ and $\beta_{\text {eff }}$ present in the data, shown as the original data in Figure 8. As may be seen, there is a substantial amount of scatter in the data. This is expected, as the data is collected on different days, at different wind speeds, and possibly with different sail shapes (due to their flexible nature). Chauvenet's criterion is applied to the data every $0.5^{\circ}$ of $\beta_{\text {eff }}$ to remove outliers in the data, and the remaining data is also shown in Figure 8. After reduction, the remaining means and their corresponding errors are now plotted in Figure 9. The errors are calculated by dividing the standard deviation of the sample by the square root of the sample size in each set of $0.5^{\circ} \beta_{\text {eff }}$.

Now, we have a curve to represent one of the unknowns in Eq. (3.42) and the only unknown left is $X_{S \delta b}^{\prime}$, which is found using the data points $\left(\beta_{e f f}, X_{S}^{\prime}, \Delta \delta_{b 0}\right)$. Again, Chauvenet's criterion is used to reduce the data, resulting in the means and errors are shown in Figure 10. 

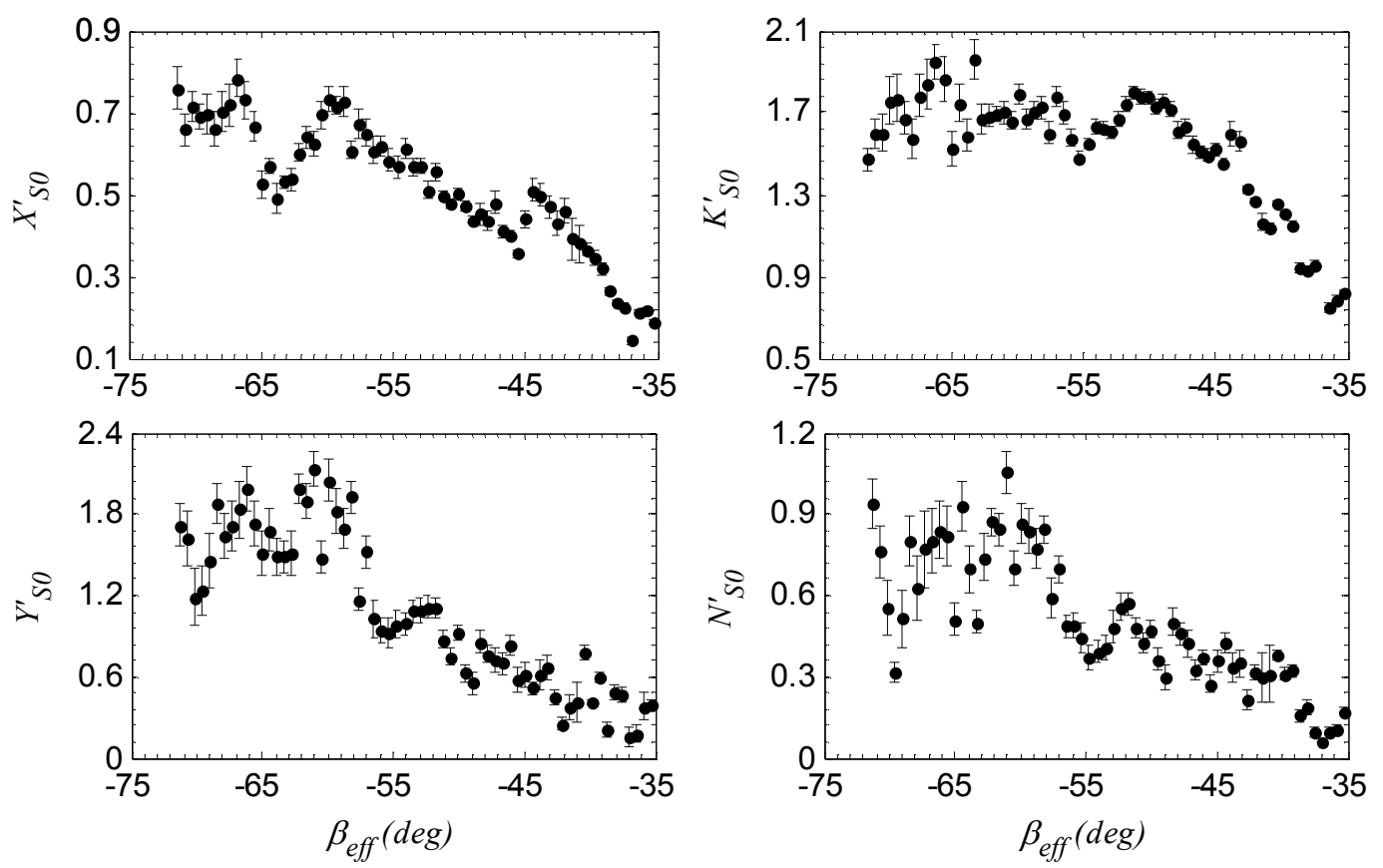

Figure 9: Measured optimal sail coefficients in 4 DOF for Avanti

The complete data presented in this study is from several different days sailing in wind speeds where the full main sail and jib were the appropriate sail choice. Thus, the wind speed, surface conditions, and trim of the boat were different among the data sets. Therefore, it is not surprising to find outliers and scatter in the data because there are so many variables that are difficult to control among experiments. Recently it has also been shown that the fluid structure interaction of the flexible sails and hull produces a substantial hysteresis effect upon the aerodynamic driving and side force coefficients when the yacht is subjected to harmonic pitching motion (Augier et al., 2013). Despite these difficulties, after the data is reduced according to a well-known and widely used statistical test, reasonable trends appear. The curves for $X_{S 0}^{\prime}$ follow the trends to be found in many sources that report sail force coefficients (Heikki Hansen, 2006; Masuyama et al., 2009; Masuyuma et al., 1993). The new component of the model, $X_{S \delta b}^{\prime}$, looks to be relatively constant with $\beta_{\text {eff. }}$. However, the values obtained for $X_{S \delta b}^{\prime}$ contain larger errors than the values for $X_{S 0}^{\prime}$, particularly in sway and yaw. It is not surprising to find error in sway, as the tests are all performed at different heel angles, and it is possible the generalization of $\beta_{\text {eff }}$ is not an adequate method of accounting for changes in heel angle. Overall, the results of the section have validated the new aerodynamic model relating physical sail deflection to the system. 

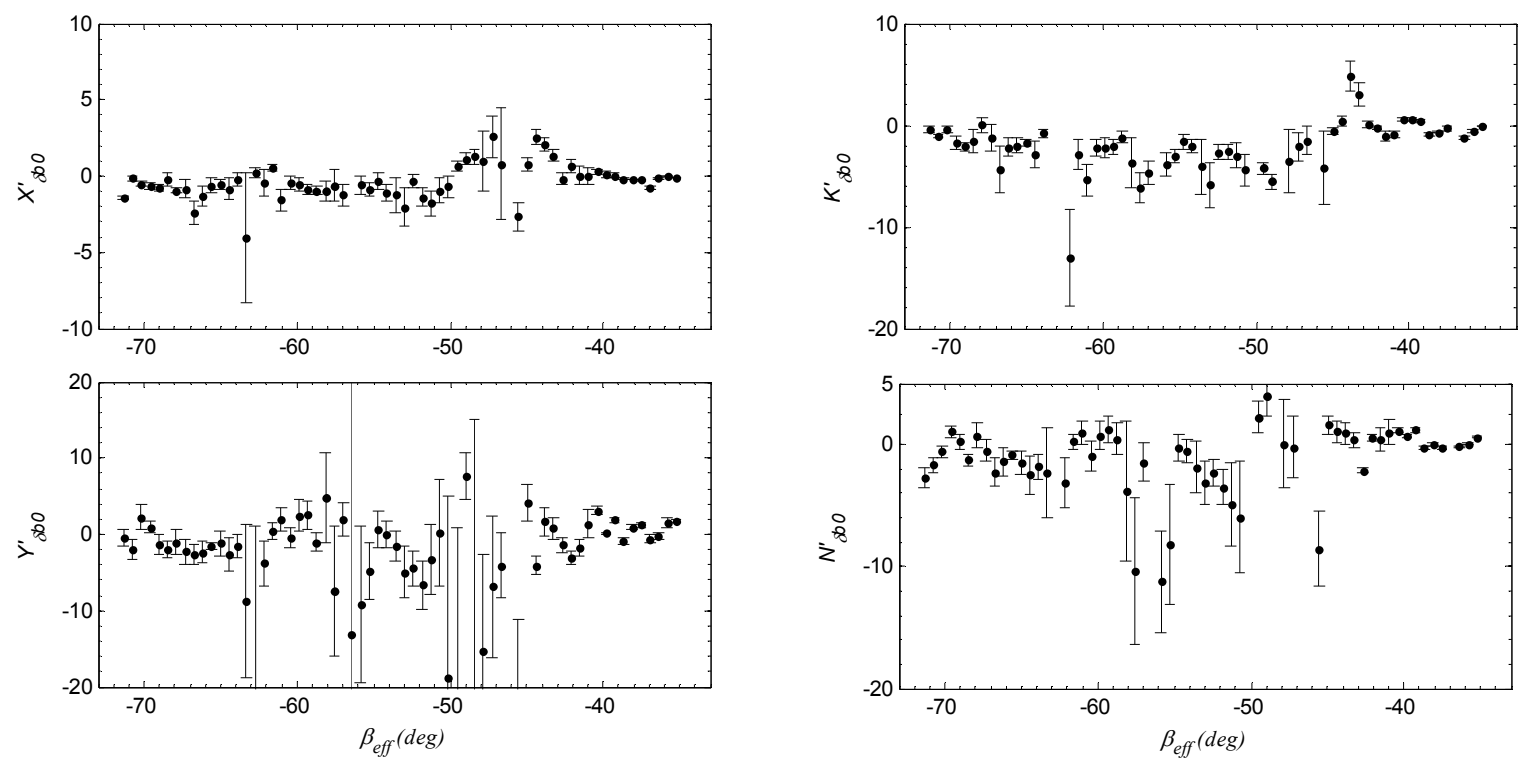

Figure 10: Measured aerodynamic derivatives for $A$ vanti

\subsection{Implementation of Non-linear Model in MATLAB/Simulink}

A nonlinear simulator based on a different yacht was developed in the MATLAB/Simulink environment. This simulator has been developed to generate data with which to test estimation techniques and algorithms, as a way to investigate the effectiveness of the maneuvers collected full-scale, and a way to test the possibility of linearizing the sailing yacht system. The simulator is based on a model and set of parameters previously published for a $10 \mathrm{~m}$ racer/cruiser (Masayuma \& Fukasawa, 2011). This section will detail the model used for simulation, the physical parameters used in the model, and present some of the data generated by the simulations.

The 4DOF equations of motion utilized by the simulation are given by Eqs. (3.45) - (3.48) (Masayuma \& Fukasawa, 2011):

$$
\begin{gathered}
\left(m+m_{x}\right) \dot{U}-\left(m+m_{y} \cos ^{2} \phi+m_{z} \sin ^{2} \phi\right) V R=X_{0}+X_{H y d r o}+X_{R u d}+X_{S} \\
\left(m+m_{y} \cos ^{2} \phi+m_{z} \sin ^{2} \phi\right) \dot{V}+\left(m+m_{x}\right) U R+2\left(m_{z}-m_{y}\right) \sin \phi \cos \phi \cdot V P \\
=Y_{H y d r o}+Y_{R u d}+Y_{S} \\
\left(I_{x x}+J_{x x}\right) \dot{P}-\left(I_{y y}+J_{y y}-I_{z z}+J_{z z}\right) \sin \phi \cos \phi \cdot R^{2} \\
=K_{H y d r o}+K_{R u d}+K_{S}-m g \overline{G M} \sin \phi
\end{gathered}
$$




$$
\begin{gathered}
\left(\left(I_{y y}+J_{y y}\right) \sin ^{2} \phi+\left(I_{z z}+J_{z z} \cos ^{2} \phi\right)\right) \dot{R}+2\left(I_{y y}+J_{y y}-I_{z z}-J_{z z}\right) \sin \phi \cos \phi \cdot P R \\
=N_{H y d r o}+N_{R u d}+N_{S}
\end{gathered}
$$

Where $m_{x}, m_{y}, m_{z}, J_{x x}, J_{y y}$, and $J_{z z}$ represent added masses and moments of inertia. Eqs. (3.45) through (3.48) with the published parameters for this yacht can be simplified to Eqs. (3.2) through (3.9). The reason the full form is used for simulation is to maintain consistency of the parameters for the sample yacht, as they were fit to the non-linear model represented by Eqs. (3.45) through (3.48). For the simulation the forces are as defined by Eqs. (3.10) - (3.16), (3.38), and Eqs. (3.18) - (3.21). The physical dimensions of the yacht, the mass parameters, and the hydrodynamic derivatives used for this simulation are found in Table 2. The aerodynamic parameters are interpolated from the curves in Figure 5, and the upright frictional drag term, $X_{0}$, is interpolated from (Masayuma, Nakamura, Tatano, \& Takagi, 1993). 
Table 2: Parameters for $10 \mathrm{~m}$ Racer/cruiser simulation

\begin{tabular}{r|rr}
\hline \multicolumn{3}{|c|}{ Mass Parameters } \\
\hline$m$ & 4410 & $\mathrm{~kg}$ \\
$m_{x}$ & 160 & $\mathrm{~kg}$ \\
$m_{y}$ & 2410 & $\mathrm{~kg}$ \\
$m_{z}$ & 12000 & $\mathrm{~kg}$ \\
$I_{x x}$ & 17700 & $\mathrm{~kg}-\mathrm{m}^{2}$ \\
$I_{y y}$ & 33100 & $\mathrm{~kg}-\mathrm{m}^{2}$ \\
$I_{z z}$ & 17200 & $\mathrm{~kg}-\mathrm{m}^{2}$ \\
$J_{x x}$ & 15300 & $\mathrm{~kg}-\mathrm{m}^{2}$ \\
$J_{y y}$ & 42400 & $\mathrm{~kg}-\mathrm{m}^{2}$ \\
$J_{z z}$ & 6700 & $\mathrm{~kg}-\mathrm{m}^{2}$ \\
\hline
\end{tabular}

\begin{tabular}{|c|c|c|c|c|}
\hline \multicolumn{5}{|c|}{ Hydrodynamic Derivatives } \\
\hline$X_{0}$ & \multicolumn{2}{|l|}{$\sim$} & $K_{v}$ & $2.80 \mathrm{E}-01$ \\
\hline$X_{v v}$ & \multicolumn{2}{|c|}{$3.38 \mathrm{E}-01$} & $K_{\phi}$ & $3.36 \mathrm{E}-03$ \\
\hline$X_{\phi \phi}$ & \multicolumn{2}{|c|}{$1.40 \mathrm{E}-03$} & $K_{v \phi \phi}$ & $-4.07 \mathrm{E}-01$ \\
\hline$X_{v v v v}$ & \multicolumn{2}{|l|}{-1.84} & $K_{v v \phi}$ & $2.24 \mathrm{E}-01$ \\
\hline \multirow[t]{2}{*}{$X_{v r}$} & \multicolumn{2}{|c|}{$-1.91 \mathrm{E}-02$} & $K_{v v v}$ & $-1.38 \mathrm{E}+00$ \\
\hline & & & $K_{p}$ & $-5.88 \mathrm{E}-01$ \\
\hline$Y_{v}$ & \multicolumn{2}{|c|}{$-5.35 \mathrm{E}-01$} & & \\
\hline$Y_{\phi}$ & \multicolumn{2}{|c|}{$-5.89 \mathrm{E}-03$} & $N_{v}$ & $-3.23 \mathrm{E}-02$ \\
\hline$Y_{v \phi \phi}$ & \multicolumn{2}{|c|}{ 7.37E-01 } & $N_{\phi}$ & $-1.52 \mathrm{E}-02$ \\
\hline$Y_{v v \phi}$ & \multicolumn{2}{|c|}{$-5.53 \mathrm{E}-01$} & $N_{v \phi \phi}$ & $2.71 \mathrm{E}-04$ \\
\hline$Y_{v v v}$ & \multicolumn{2}{|c|}{$3.07 \mathrm{E}+00$} & $N_{v v \phi}$ & $-9.06 \mathrm{E}-02$ \\
\hline$Y_{p}$ & \multicolumn{2}{|c|}{ 2.19E-01 } & $N_{v v v}$ & $-2.98 \mathrm{E}-02$ \\
\hline \multirow[t]{6}{*}{$Y_{r}$} & \multicolumn{2}{|c|}{$-4.01 \mathrm{E}-03$} & $N_{r}$ & $-5.89 \mathrm{E}-03$ \\
\hline & \multicolumn{4}{|c|}{ Rudder Parameters } \\
\hline & $C x_{\delta r}$ & \multicolumn{3}{|c|}{$-3.79 \mathrm{E}-02$} \\
\hline & $C y_{\delta r}$ & \multicolumn{3}{|c|}{$-1.80 \mathrm{E}-01$} \\
\hline & $C k_{\delta r}$ & \multicolumn{3}{|c|}{$9.76 \mathrm{E}-02$} \\
\hline & $C n_{\delta r}$ & \multicolumn{3}{|c|}{$9.74 \mathrm{E}-02$} \\
\hline
\end{tabular}

A block diagram depicting the simulation is shown in Figure 11. The details of the boat model are contained in the block with the picture of the sailing yacht. As shown, the model requires five inputs to run. The states begin with the initial conditions found by balancing forces and moments for the given true wind. While the model is simulating, it is assumed that the true wind speed and direction in the Earth-fixed frame are constant. Apparent wind speed and direction are calculated at each step in the calculation based on the boat velocity and heading, and are included in the output, $y$, of the model. Heading during the simulation is calculated by integrating the kinematic relationship: 


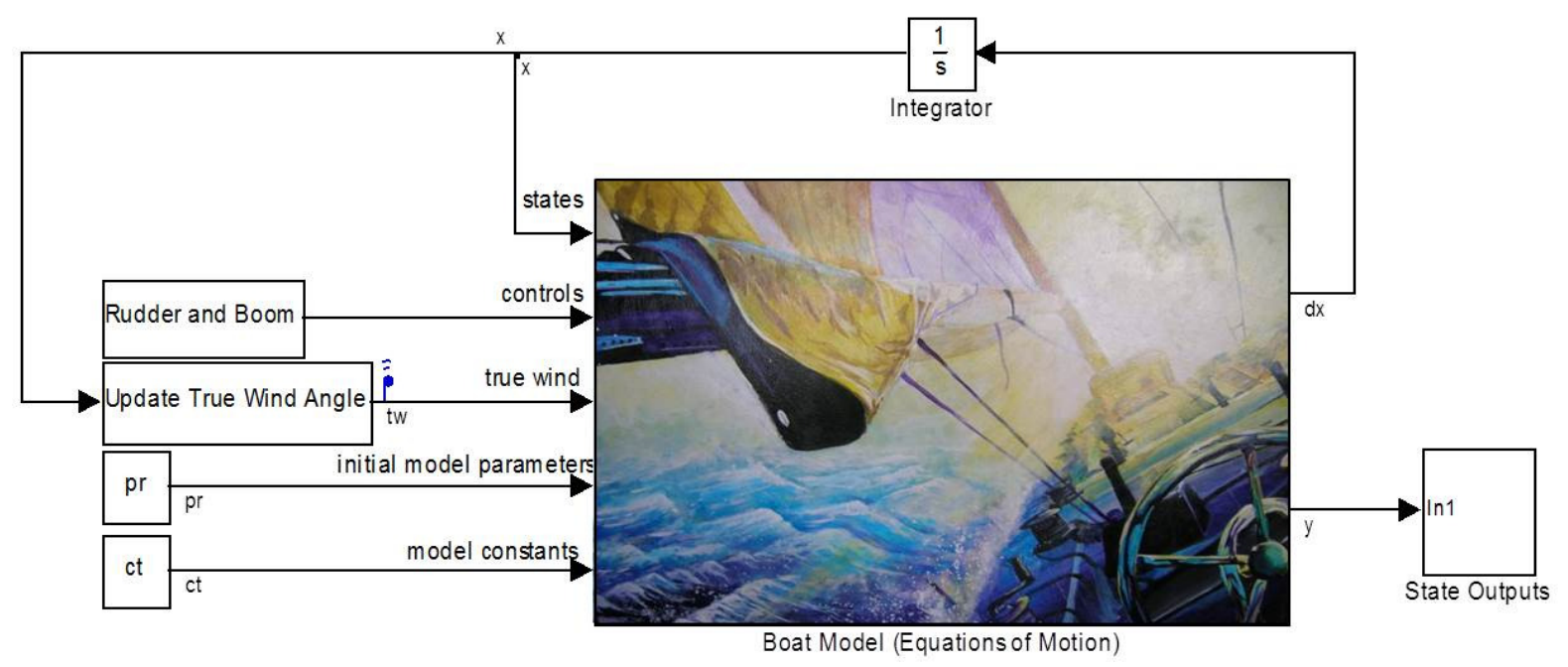

Figure 11: Simulink block diagram of non-linear simulation.

$$
\dot{\psi}=R \cos \phi
$$

The input ' $p r$ ' represents the parameter vector $\bar{\beta}$, and ' $c t$ ' represents a vector of model constants such as mass, air density, etc. The aerodynamic force model which is evaluated within the boat block contains lookup tables which choose the correct aerodynamic coefficients based on the apparent wind speed and direction. This model assumes that the sails are always set in the optimal position for the given conditions, and thus the model is known to have a discrepancy fort what actually occurs during full-scale tests. The hydrodynamic model also contains a lookup table for returning upright hull drag $X_{0}$, based on forward velocity, $U$.

The results of simulating the yacht model are compared to responses seen on the water to make sure the model is trending well for a monohull sailing yacht. While the Precision 23 is a different boat than the $10 \mathrm{~m}$ racer/cruiser, the general behavior of the two should be similar. The difference will be in the magnitude and timing of the responses. The response to a singlet input is shown in Figure 12. Included in the time history is the measurement or calculation of apparent wind speed and direction. For both cases, the boats are shown sailing upwind on a starboard tack. The simulation results are for a true wind speed of $5 \mathrm{~m} / \mathrm{s}$ and true wind direction of $45 \mathrm{deg}$. The real data is taken on a day where the average true wind speed is $6.2 \mathrm{~m} / \mathrm{s}$. 

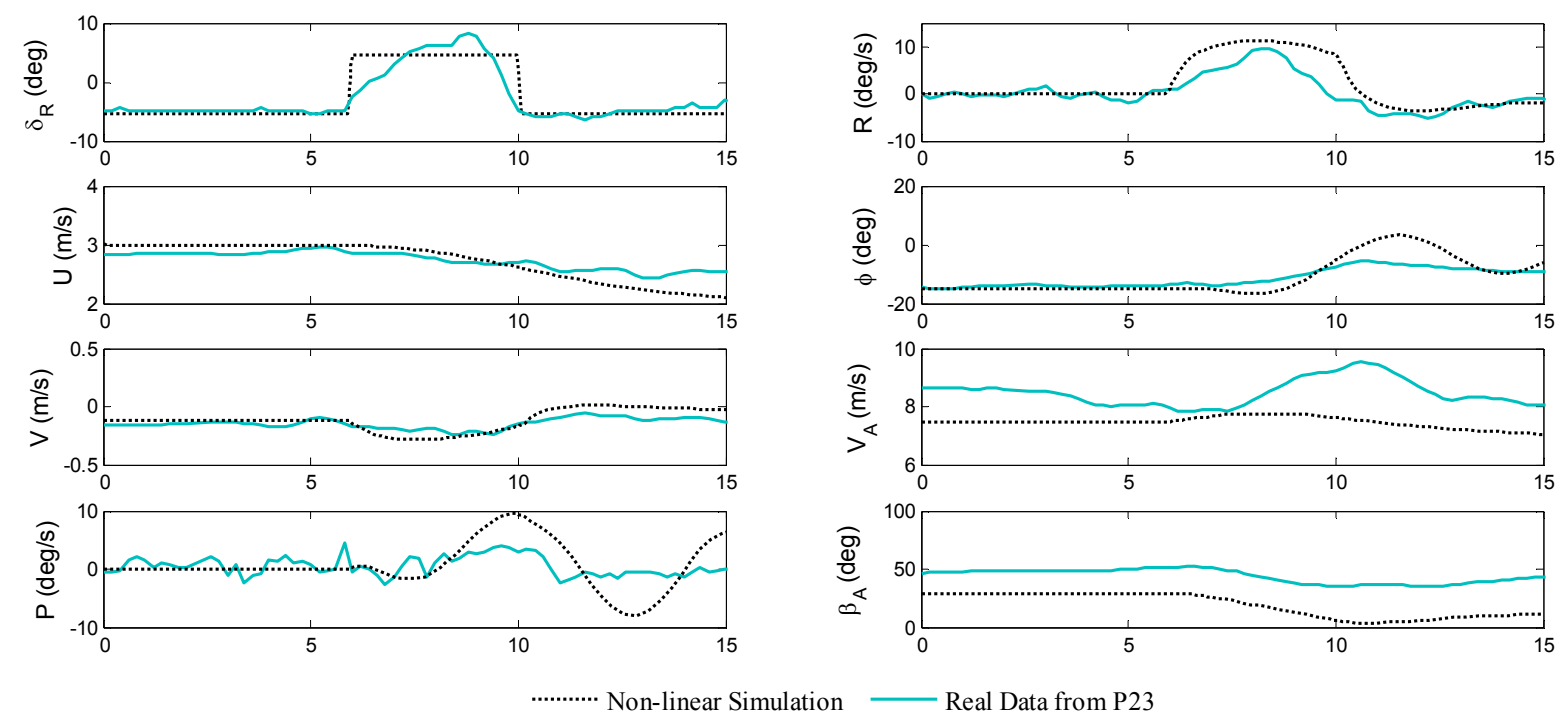

Figure 12: Measured and simulated response to a rudder singlet.

Even though the simulation represents a very different boat than the P23, the dynamic response to the rudder input is very similar, even in rate and magnitude. In the simulation, $P$ and $\phi$ tend to oscillate, this is due again to errors between the model and real life which accumulate as the simulated model propagates forward in time. After looking at several comparisons such as those shown in Figure 12 the author has decided that this non-linear model is a suitable starting point for the rest of the study. 


\section{$4 \quad$ Linear Dynamic Sailing Yacht Models}

A linearized model has several benefits. Linear models are easier and thus more straightforward to estimate than non-linear models, especially with methods like least squares and maximum likelihood estimation. Linear time invariant models exhibit first or second order oscillatory modes, which are characterized by the eigenvalues of the $A$ matrix in Eq (4.1). With appropriate inputs to excite and record the dynamic response of these modes, estimation can be very quick and accurate from a relatively small sample of data. In addition to making the process of estimation easier, there is a significant inventory of control system design tools for linear

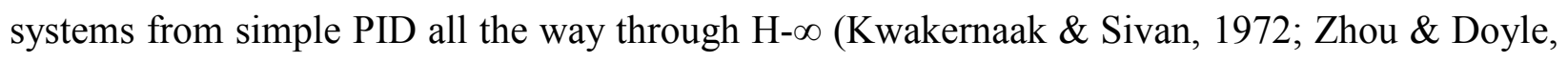
1997). The following Section 4.1 describes in more detail the theory of linear systems analysis.

\subsection{Linear Dynamic Systems Theory}

Many control and estimation techniques work even better on a linearized state space model, a fact which the aerospace industry has effectively exploited. The linearization of aircraft equations of motion is what made the early successes of aircraft estimation and control system development possible. The equations of motion of an aircraft are nonlinear. To linearize them, the equations are evaluated around a nominal trajectory or trim point. For an aircraft, this could mean straight and level flight at a pre-determined airspeed, angle of attack, and altitude. The inherent assumption made during linearization is that deviations from the nominal condition are small and will not have a large effect on overall system behavior. The result is a linear state space model of the form:

$$
\begin{aligned}
\dot{\vec{x}}(t) & =A \vec{x}(t)+B \vec{u}(t)+F \vec{w}(t), \quad \vec{x}\left(t_{0}\right)=\vec{x}_{0} \\
\vec{y}(t) & =C \vec{x}(t)+D \vec{u}(t)
\end{aligned}
$$

The $F$ matrix and $w$ vector are the disturbance/noise terms. In this work, the disturbance terms are not considered, but in future analysis it would be possible to include the effects of wind in these terms, where $\vec{w}=\left[\begin{array}{ll}V_{T} & \beta_{T}\end{array}\right]^{T}$. What is left then is the following state space model:

$$
\begin{aligned}
\dot{\vec{x}}(t) & =A \vec{x}(t)+B \vec{u}(t), \quad \vec{x}\left(t_{0}\right)=\vec{x}_{0} \\
\vec{y}(t) & =C \vec{x}(t)+D \vec{u}(t)
\end{aligned}
$$


$A, B, C$, and $D$ are the system Jacobian matrices evaluated around the nominal condition $x_{0}$, and $u_{0}$, and are defined as:

$$
\begin{aligned}
& A=\frac{\partial f[\vec{x}, \vec{u}, \vec{\beta}]}{\partial \vec{x}}, \quad B=\frac{\partial f[\vec{x}, \vec{u}, \vec{\beta}]}{\partial \vec{u}} \\
& C=\frac{\partial g[\vec{x}, \vec{u}, \vec{\beta}]}{\partial \vec{x}}, \quad D=\frac{\partial g[\vec{x}, \vec{u}, \vec{\beta}]}{\partial \vec{u}}
\end{aligned}
$$

where $f$ and $g$ are the nonlinear functions of the states and controls described in Eq.(3.1). The system matrices can be obtained by either symbolically differentiating the non-linear state equations or numerical estimattion using the central difference formula (Jategaonkar, 2006; Stengel, 2004). The non-linear model presented in Chapter 3 is numerically linearized and simulated in Section 4.3.

The state space model listed in Eq. (4.2) is defined in the time domain. It is very useful to analyze linear models in the frequency domain, a transformation performed with Laplace transforms. The definition of a Laplace transform to take a function from the time domain to the frequency domain is given in Eq. (4.4), and an inverse Laplace transform to take the function back to the time domain is given in Eq. (4.5).

$$
\begin{gathered}
\mathscr{L}[x(t)]=x(s)=\int_{0}^{\infty} x(t) e^{-s t} d t \\
\mathscr{L}^{-1}[x(s)]=x(t)=\frac{1}{2 \pi j} \int_{\sigma+j \infty}^{\sigma-j \infty} x(s) s^{s t} d s
\end{gathered}
$$

The Laplace transformation is a linear operation and thus has the following properties:

$$
\begin{gathered}
\mathscr{L}\left[x_{1}(t)+x_{2}(t)\right]=\mathscr{L}\left[x_{1}(t)\right]+\mathscr{L}\left[x_{2}(t)\right]=x_{1}(s)+x_{2}(s) \\
\mathscr{L}[c x(t)]=c \mathscr{L}[x(t)]=c x(s)
\end{gathered}
$$

The Laplace transformation of a time derivative is:

$$
\mathscr{L}[\dot{x}(t)]=\dot{x}(s)=s x(s)-x(0)
$$

Where $x(0)$ is the value of $x$ at $s=0$. Applying the properties described in Eq. (4.4) - (4.8) to the state equation defined in Eq. (4.2) and re-arranging the terms results in: 


$$
\begin{aligned}
\mathscr{L}[\dot{\bar{x}}(t)] & =\mathscr{L}[A \vec{x}(t)+B \vec{u}(t)] \\
s \vec{x}(s)-x(0) & =A \vec{x}(s)+B \vec{u}(s) \\
(s I-A) \vec{x}(s) & =x(0)+B \vec{u}(s)
\end{aligned}
$$

It should be noted this treatment of linear systems has been for linear time invariant (LTI) systems, meaning the $A$ and $B$ matrices to do no change with time. If they did, the Laplace transformation would be applied to each element of the $A$ and $B$ matrices as well. Therefore, in the Laplace frequency domain, the states are related to the controls by the following equation:

$$
\vec{x}(s)=(s I-A)^{-1}[x(0)+B \vec{u}(s)]
$$

The characteristic matrix of $A$ then can be defined as:

$$
K(s) \triangleq(s I-A)^{-1}
$$

A typical entry of the characteristic matrix is a ratio of polynomial functions of $s$ :

$$
k_{i j}(s)=\frac{c_{i j}\left(s^{n-1}+b_{n-2} s^{n-2}+\cdots+b_{1} s+b_{0}\right)}{s^{n}+d_{n-1} s^{n-1}+\cdots+d_{1} s+d_{0}} \quad i, j=1,2 \ldots n
$$

Which may be written in factored form:

$$
k_{i j}(s)=\frac{c_{i j}\left(s-\beta_{1}\right)\left(s-\beta_{2}\right) \cdots\left(s-\beta_{n-1}\right)}{\left(s-\lambda_{1}\right)\left(s-\lambda_{2}\right) \cdots\left(s-\lambda_{n}\right)}
$$

Here $\lambda_{1}, \lambda_{2}, \ldots \lambda_{n}$ by definition are the eigenvalues of the original $A$ matrix. Looking at the effect on the state, the equation for the first of the $n$ states becomes:

$$
\begin{aligned}
x_{1}(s) & =k_{i 1} x_{1}(0)+k_{i 2} x_{2}(0)+\cdots+k_{i n} x_{n}(0) \\
& \triangleq \frac{N_{i}(s)}{D(s)}
\end{aligned}
$$

Where $N_{i}$ is a linear combination of the polynomial numerators in $K(s)$ where each is weighted according to the initial conditions. $D(s)$ is the common denominator for all the polynomial expressions of $K(s)$, and contains the eigenvalues of the $A$ matrix. The ratio defined in Eq. (4.14) can be re-written using partial fraction expansion as: 


$$
\frac{N_{i}(s)}{D(s)}=\frac{N_{1}}{s-\lambda_{1}}+\frac{N_{2}}{s-\lambda_{2}}+\cdots+\frac{N_{n}}{s-\lambda_{n}}
$$

The individual terms of Eq. (4.15) are called the modes of response to the initial condition $x(0)$. The time response corresponding with each mode can be determined by taking the inverse Laplace transform back into the time domain.

$$
\mathscr{L}^{-1}\left[\frac{N_{i}}{s-\lambda_{i}}\right]=N_{i} e^{\lambda_{i} t}
$$

The elegance of linear systems analysis is evident, as the eigenvalues of the system $A$ matrix characterize the dynamic response of the vehicle. The eigenvalues can only be first order or second order. A first order response occurs when an eigenvalue is only real-valued, meaning it lies on the real axis of the complex plane, shown in Figure 13. In the case where $\lambda_{i}>0$, the time response exponentially grows, and the mode can be said to unstable. In the case where $\lambda_{\mathrm{i}}<0$, the time signal exponentially decays, and the mode can be said to be asymptotically stable. These two responses are shown in Figure 14. The first order modes may alternately be expressed with a time constant, defined in Eq. (4.17).

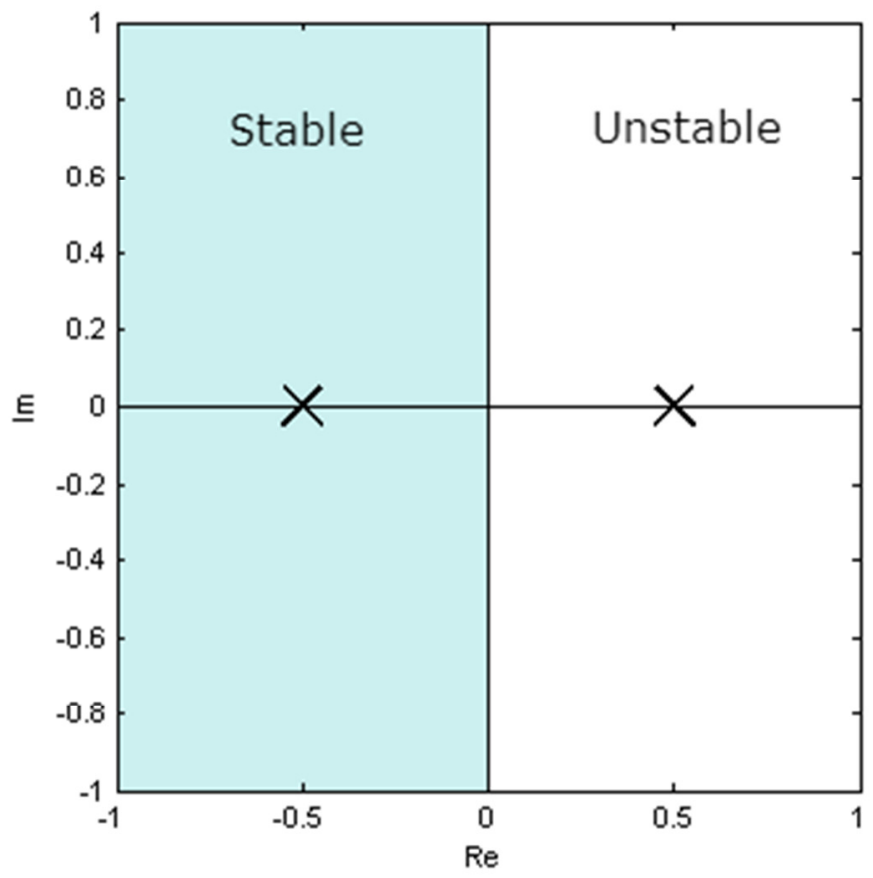

Figure 13: The complex plane showing stable first order mode at $\mathbf{- 0 . 5}$ and an unstable first order modes at 0.5 . 


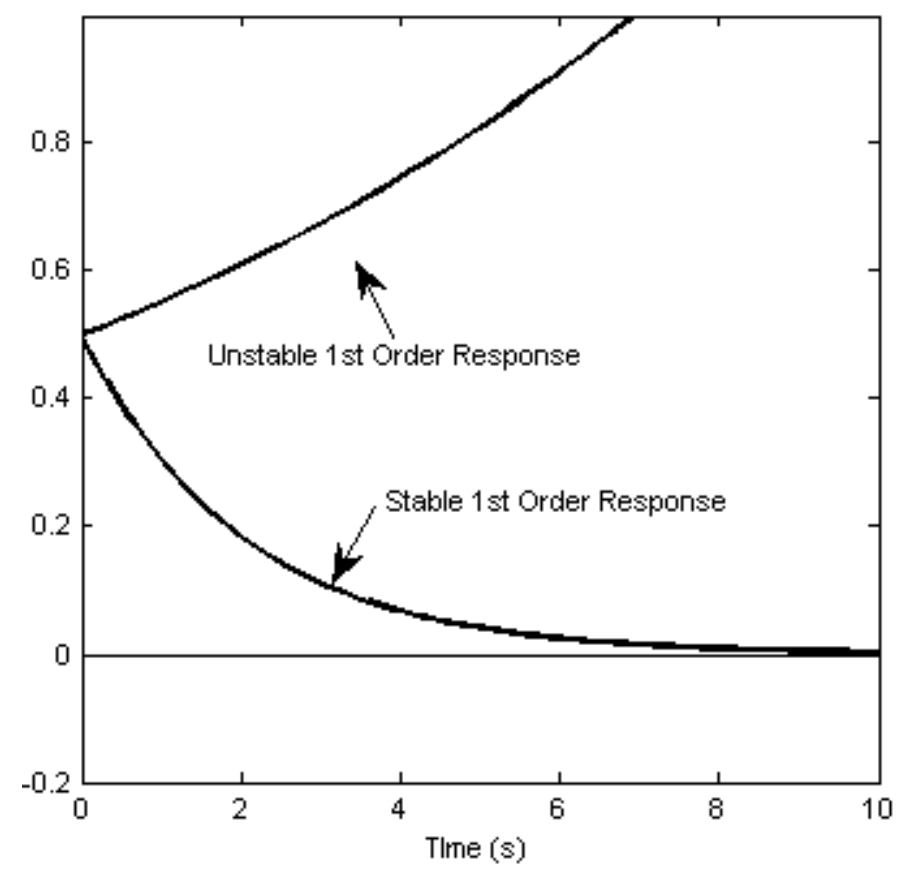

Figure 14: Time response of both stable and unstable first order modes.

$$
N_{i} e^{-t / \tau_{i}} \rightarrow \tau_{i}=-\lambda_{i}^{-1}
$$

A second order response is an oscillatory mode that occurs when an eigenvalue is a pair of complex conjugates:

$$
\lambda_{i}=\sigma \pm j \omega_{d}
$$

Where $\sigma$ is the real part of the root and $\omega_{d}$, also known as the damped frequency, is the imaginary part of the root. In this case, the eigenvalue represents two roots of the quadratic equation expressed in the Laplace domain:

$$
s^{2}-2 \sigma s+\left(\sigma^{2}+\omega_{d}^{2}\right)=s^{2}+2 \zeta \omega_{n} s+\omega_{n}^{2}
$$

Which is where the natural frequency, $\omega_{n}$ and damping ratio, $\zeta$, of the second order mode may be defined in terms of the eigenvalue components:

$$
\begin{aligned}
& \omega_{n}=\sqrt{\sigma^{2}+\omega_{d}^{2}} \\
& \zeta=\frac{-\sigma}{\sqrt{\sigma^{2}+\omega_{d}^{2}}}
\end{aligned}
$$




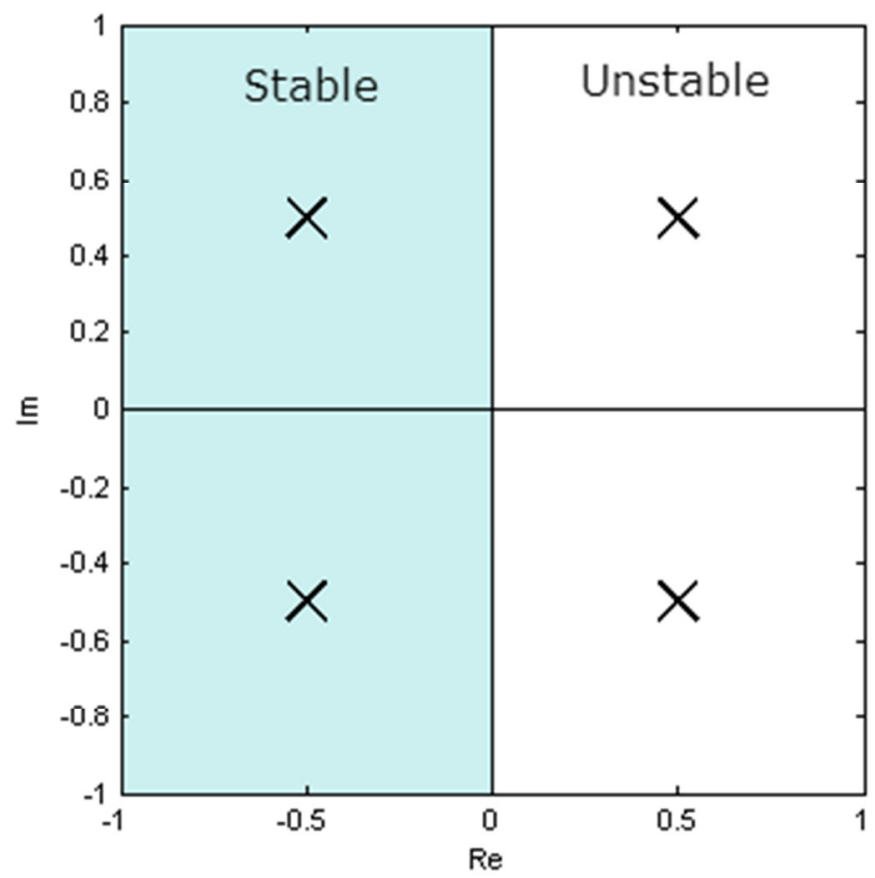

Figure 15: Complex plan showing a stable second order mode at $\mathbf{- 0 . 5 + / - 0 . 5 i}$ and an unstable second order mode at $0.5+/-0.5 i$.

If a complex eigenvalue lies on the left hand side of the imaginary axis, $\sigma<0$, then the mode is stable. If the complex eigenvalue lies on the right hand side of the imaginary axis, ie $\sigma$ $>0$, the mode is unstable. This is demonstrated in Figure 15, and the difference between stable and unstable modal oscillation is shown in Figure 16.

To put it all together, it has been shown that the time response for a linear system is the sum of individual responses which have either exponential (first order) or sinusoidal (second order) form. This fact is what makes linear system identification more straight-forward; if control inputs are isolated it becomes very easy to separate and characterize the system dynamics in terms of a combination of first and second order modes. 


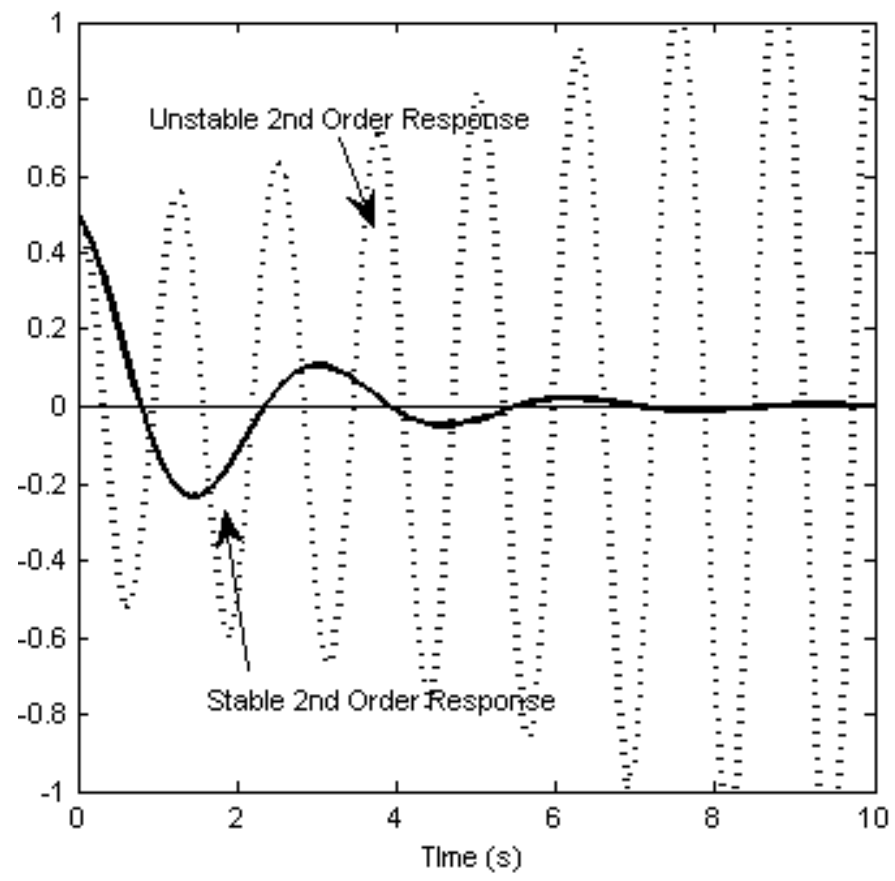

Figure 16: Time response of both stable and unstable second order modes

\subsection{Linearized Sailing Yacht Model}

Now that a generic state space linear model has been described, it will be formulated specifically for the sailing yacht. The primary assumption made for model linearization is that the linear model is valid for small perturbations around the trim point. For aircraft, the implications of this assumption are well understood, but as this is the first attempt at a linear dynamic sailing yacht model, this assumption needs further investigation. Figure 17 shows a polar diagram for a sailboat, where the radial lines represent the orientation of the boat to the true wind (true wind direction), the concentric arcs represent boat velocities, and the bold curves drawn on this diagram represent the range of true wind speeds. Each point on the true wind speed curve is then associated with a true wind direction and boat speed for which the forces and moments are in equilibrium. Although not part of a polar diagram, the roll angle of the yacht would also be defined as part of what creates equilibrium in the four degree of freedom forces and moments. For each linear model, essentially one of these points is chosen as the steady state trim value of the boat. In the following Section 4.3, a brief investigation is made into linearizing the non-linear model at varying true wind speeds and directions as based on the author's 


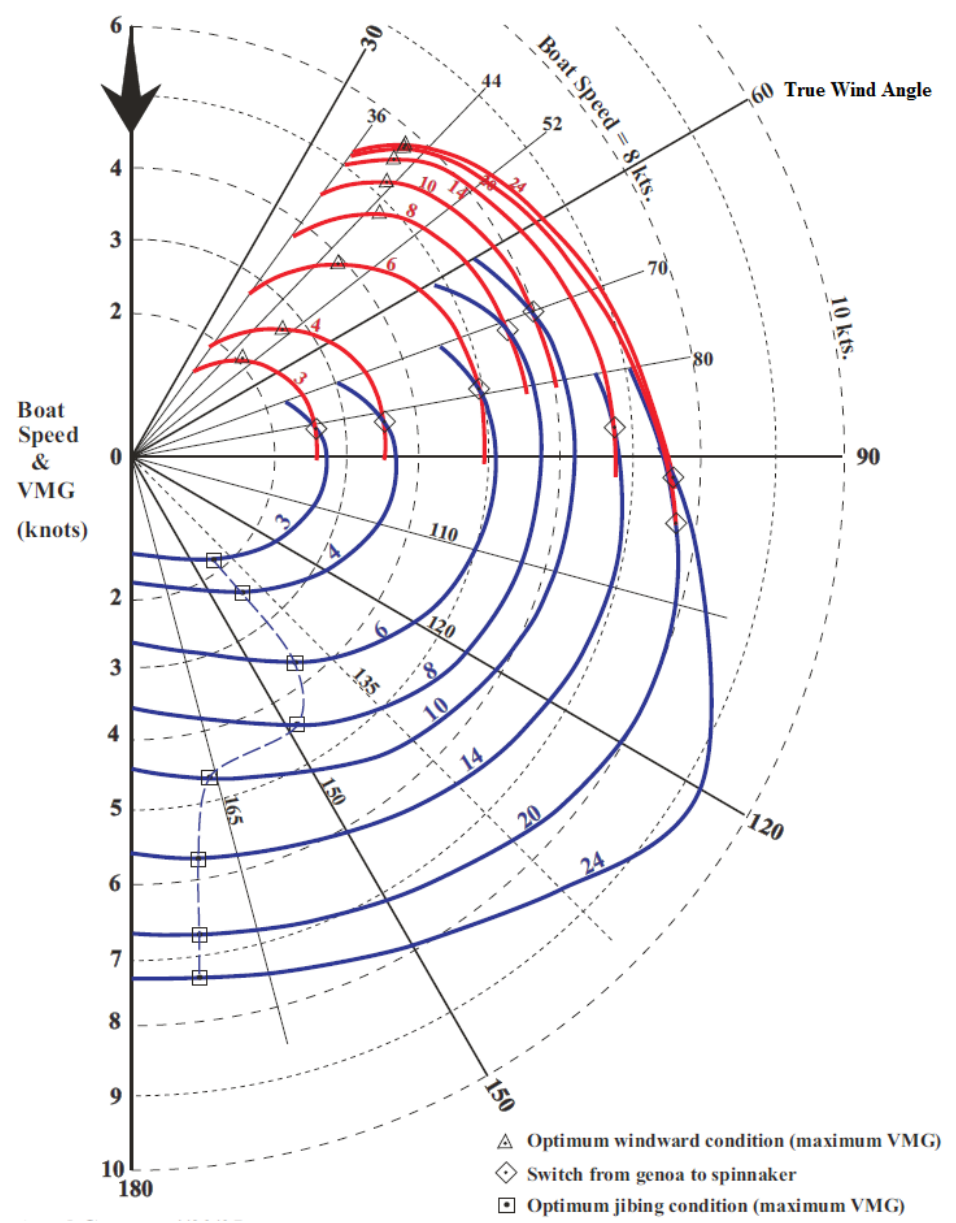

Figure 17: Sample Polar Diagram for Sailboat (Gentry, 1995).

Note this polar diagram is for the Ranger 23 sailboat, which has the same PHRF handicap rating as the Precision 23, which means this polar diagram closely represents expected performance for Avanti.

experience this will likely be the primary driver of the observed dynamics. A large part of the value in the data collected for this study is that it covers a large part of the sailing trim diagram and as such general conclusions may be formulated about how much of the trim diagram may be represented by a single linear model.

Now that a definition of a trim point for the sailing yacht dynamic model has been clarified, the details of the linear model for a given trim point may be given. The state vector for the sailing yacht is chosen to include the surge and sway velocities, roll rate, yaw rate, and roll angle. The linear model is defined in terms of perturbed variables rather than total variables. The total variables, denoted by capital letters, are the variables actually measured on the water and are defined as the sum of the perturbed variables and the trim condition, denoted by small letters: 


$$
\begin{aligned}
& U=u+\Delta u \\
& V=v+\Delta v \\
& P=p+\Delta p \\
& R=r+\Delta r \\
& \Phi=\phi+\Delta \phi \\
& \delta_{R}=\delta_{r}+\Delta \delta_{r} \\
& \delta_{B}=\delta_{b}+\Delta \delta_{b}
\end{aligned}
$$

The resulting state, control, and trim vectors are defined as:

$$
\dot{\vec{x}}=\left[\begin{array}{c}
\Delta \dot{u} \\
\Delta \dot{v} \\
\Delta \dot{p} \\
\Delta \dot{r} \\
\Delta \dot{\phi}
\end{array}\right] \quad \vec{x}=\left[\begin{array}{c}
\Delta u \\
\Delta v \\
\Delta p \\
\Delta r \\
\Delta \phi
\end{array}\right] \quad \vec{u}=\left[\begin{array}{l}
\Delta \delta_{r} \\
\Delta \delta_{b}
\end{array}\right] \quad \vec{x}_{0}=\left[\begin{array}{l}
u \\
v \\
p \\
r \\
\phi
\end{array}\right] \quad \vec{u}_{0}=\left[\begin{array}{c}
\delta_{r} \\
\delta_{b}
\end{array}\right]
$$

In order to determine the $A$ matrix, the partial derivative of each state equation from the nonlinear model reported in Eqs.(3.2) -(3.5) is taken with respect to each one of the individual states and evaluated at the trim condition. This is part of the definition of the $A$ matrix from Eq. (4.3). The same approach is used to calculate the B matrix.

$$
A=\left[\left.\begin{array}{lllll}
\frac{\partial \dot{U}}{\partial U} & \frac{\partial \dot{U}}{\partial V} & \frac{\partial \dot{U}}{\partial P} & \frac{\partial \dot{U}}{\partial R} & \frac{\partial \dot{U}}{\partial \Phi} \\
\frac{\partial \dot{V}}{\partial U} & \frac{\partial \dot{V}}{\partial V} & \frac{\partial \dot{V}}{\partial P} & \frac{\partial \dot{V}}{\partial R} & \frac{\partial \dot{V}}{\partial \Phi} \\
\frac{\partial \dot{P}}{\partial U} & \frac{\partial \dot{P}}{\partial V} & \frac{\partial \dot{P}}{\partial P} & \frac{\partial \dot{P}}{\partial R} & \frac{\partial \dot{P}}{\partial \Phi} \\
\frac{\partial \dot{R}}{\partial U} & \frac{\partial \dot{R}}{\partial V} & \frac{\partial \dot{R}}{\partial P} & \frac{\partial \dot{R}}{\partial R} & \frac{\partial \dot{R}}{\partial \Phi} \\
\frac{\partial \dot{\Phi}}{\partial U} & \frac{\partial \dot{\Phi}}{\partial V} & \frac{\partial \dot{\Phi}}{\partial P} & \frac{\partial \dot{\Phi}}{\partial R} & \frac{\partial \dot{\Phi}}{\partial \Phi}
\end{array}\right|_{\bar{x}=\bar{x}_{0}} \quad B=\left[\begin{array}{ll}
\frac{\partial \dot{U}}{\partial \delta_{R}} & \frac{\partial \dot{U}}{\partial \delta_{B}} \\
\frac{\partial \dot{V}}{\partial \delta_{R}} & \frac{\partial \dot{V}}{\partial \delta_{B}} \\
\frac{\partial \dot{P}}{\partial \delta_{R}} & \frac{\partial \dot{P}}{\partial \delta_{B}} \\
\frac{\partial \dot{R}}{\partial \delta_{R}} & \frac{\partial \dot{R}}{\partial \delta_{B}} \\
\frac{\partial \dot{\Phi}}{\partial \delta_{R}} & \frac{\partial \dot{\Phi}}{\partial \delta_{B}}
\end{array}\right]_{\bar{x}=\bar{x}_{0}}\right.
$$

\subsection{Numerical Linearization of 10m Sailing Yacht Based on Non-Linear Model}

Analytically defining the linear model from the non-linear equations of motion for the sailing yacht is a fairly rigorous process. When analysis with this linear model structure began, it was uncertain how many different models would be constructed using different definitions of 
state and control vectors, the author opted for numerically linearizing the non-linear simulation which was developed in Section 3.2. Using the physics based model and parameters presented in (Masayuma \& Fukasawa, 2011), six trim conditions for the $10 \mathrm{~m}$ racer/cruiser presented in Section 3.5 are found by balancing the forces and moments. These represent two true wind speeds, 5 and $6 \mathrm{~m} / \mathrm{s}$, and three true wind angles, $45^{\circ}, 60^{\circ}$, and $90^{\circ}$, representing sailing from upwind through a beam reach. These six trim conditions are used as the initial conditions for the simulation presented later in this section.

It is not uncommon for several linear models to be identified for a single aircraft, and the controller is designed to switch between models when conditions change and favor one model over others. The questions for a sailing yacht are how many of those models would be necessary, and for what range of true wind speed and direction would they remain valid. As linear models are much more straightforward to identify, the author was very interested in this question, and used six balanced trim conditions to investigate. The nonlinear model presented in Section 3.5 is linearized using the central difference theorem with a perturbation size of $0.01 \%$ of the trimmed value. This results in six linear state space models, at two true wind speeds and three true wind angles. One of the models is shown in Eq. (4.25):

$$
\begin{aligned}
& V_{T}=5 \mathrm{~m} / \mathrm{s} \quad \beta_{T}=45^{\circ} \\
& \vec{x}_{0}=\left[\begin{array}{lllll}
3.0 \frac{\mathrm{m}}{\mathrm{s}} & -0.12 \frac{\mathrm{m}}{\mathrm{s}} & 0.00 \frac{\mathrm{rad}}{\mathrm{s}} & 0.00 \frac{\mathrm{rad}}{\mathrm{s}} & -0.27 \mathrm{rad}
\end{array}\right]^{T} \quad \vec{u}_{0}=[-0.10 \mathrm{rad}] \\
& {\left[\begin{array}{c}
\dot{u} \\
\dot{v} \\
\dot{p} \\
\dot{r} \\
\dot{\phi}
\end{array}\right]=\left[\begin{array}{ccccc}
-0.156 & -0.244 & 0 & -0.285 & 0.055 \\
0.130 & -0.689 & -0.077 & 0.874 & -0.049 \\
-0.085 & 0.325 & 0 & -0.700 & -1.972 \\
-0.031 & -1.471 & 0 & -3.511 & -0.445 \\
0 & 0 & 1 & 0 & 0
\end{array}\right]\left[\begin{array}{c}
\Delta u \\
\Delta v \\
\Delta p \\
\Delta r \\
\Delta \phi
\end{array}\right]+\left[\begin{array}{c}
0.087 \\
-1.840 \\
0.473 \\
2.386 \\
0
\end{array}\right]\left[\Delta \delta_{R}\right]}
\end{aligned}
$$



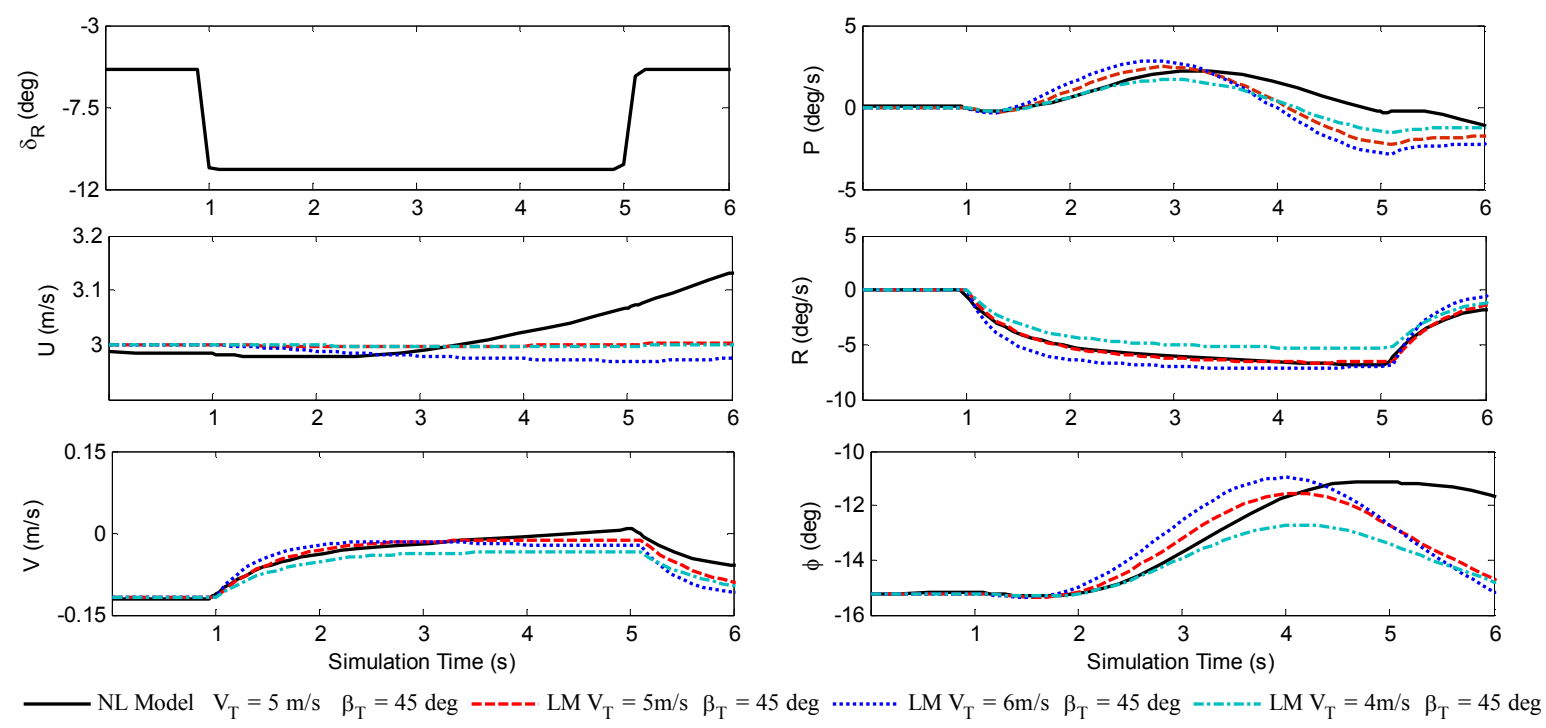

Figure 18: Linear model response to rudder singlet at varying true wind speeds.

The $C$ matrix is a $5 \times 5$ identity matrix, and the $D$ matrix is [0]. The $\Delta$ 's indicate that the model is for perturbation values of the states, that is, deviations away from the trim state, $x_{0}$. When the above model is simulated, the trim values must be added to the states output by the model. This is shown mathematically by the perturbation equations in Eq. (4.22). This linear model includes only the rudder as a control because the parameters published for this $10 \mathrm{~m}$ yacht only include the optimal sail coefficients and not the new aerodynamic parameters introduced by the model in Section 3.4.2.

Figure 18 shows the response of the models to a rudder singlet. The solid black lines represent the response given by a simulation of the full non-linear system at $V_{T}=5 \mathrm{~m} / \mathrm{s}$ and $\beta_{T}=$ $45^{\circ}$. This is the point at which the model in Eq. (4.25) has been linearized. The response of this system is shown in Figure 18 as the red dashed line. The other two dashed lines are the response to the same input of two different models which were linearized about points of constant $\beta_{T}=$ $45^{\circ}$, but differing true wind speed, $V_{T}=4 \mathrm{~m} / \mathrm{s}(7.8 \mathrm{kts})$ and $6 \mathrm{~m} / \mathrm{s}(11.7 \mathrm{kts})$. All three linear models show good behavior quantified by a fit to the original simulation data of $R^{2}>0.97$ their ability to closely reproduce the time history generated by the non-linear model. Errors introduced by the linearization tend to accumulate enough to affect a simulation after $5 \mathrm{~s}$, and can be significant by 

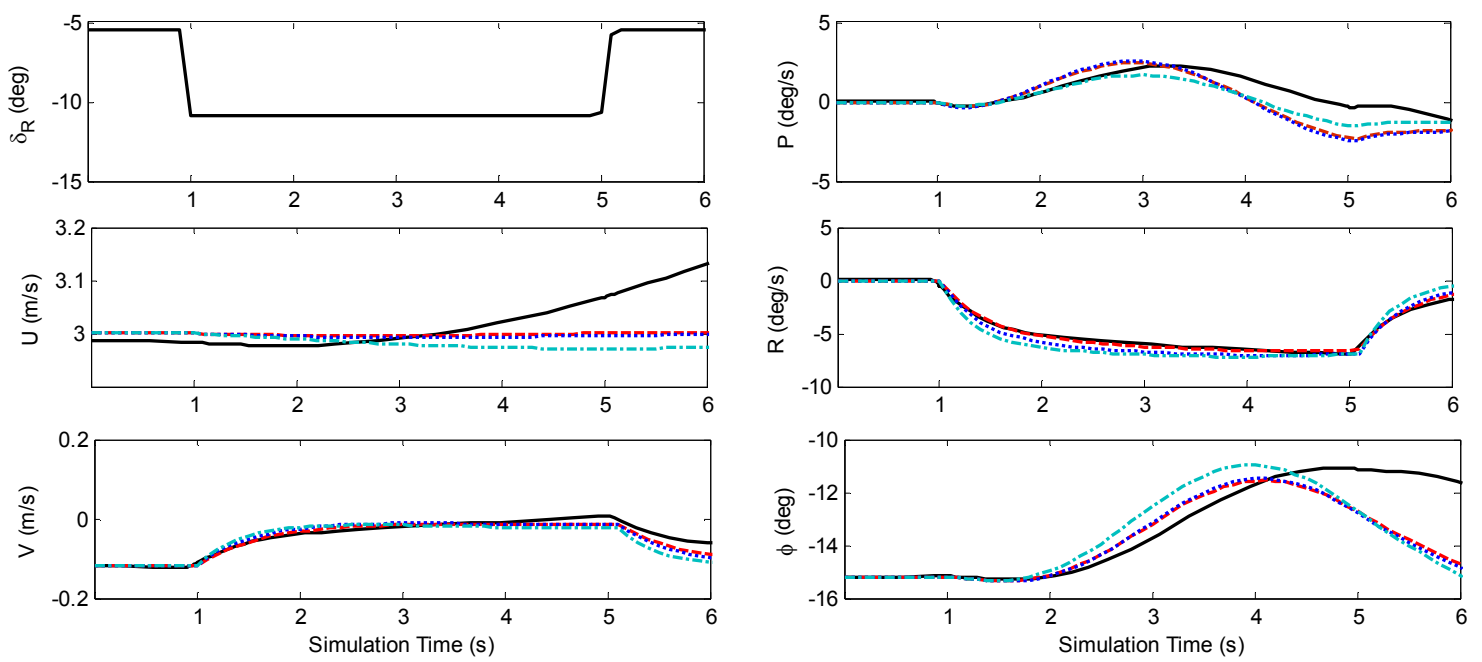

NL Model $\mathrm{V}_{\mathrm{T}}=5 \mathrm{~m} / \mathrm{s} \quad \beta_{\mathrm{T}}=45 \mathrm{deg}-----\cdot \mathrm{LM} \mathrm{V}_{\mathrm{T}}=5 \mathrm{~m} / \mathrm{s} \quad \beta_{\mathrm{T}}=45 \mathrm{deg}$

$\mathrm{LM} \mathrm{V}_{\mathrm{T}}=5 \mathrm{~m} / \mathrm{s} \quad \beta_{\mathrm{T}}=60 \mathrm{deg}-\cdot-\cdot \cdot-\mathrm{LM} \mathrm{V}_{\mathrm{T}}=5 \mathrm{~m} / \mathrm{s} \quad \beta_{\mathrm{T}}=90 \mathrm{deg}$

Figure 19: Linear model response to rudder singlet at varying true wind directions.

10s of propagation forward in time, so the difference between the non-linear model and linear models by the time the simulation gets to $12 \mathrm{~s}$ is expected.

Figure 19 shows the same information as Figure 18, but with varying true wind angles, from $\beta_{T}=45^{\circ}-90^{\circ}$, representing trimmed sailing from upwind through a beam reach. Again, the linear models are doing a good job of handling the change in true wind direction with each of the three model responses in this case fitting the simulation data with $R^{2}>0.98$. The results of Figure 18 and Figure 19 are very promising because they indicate that the same linear model could be used for this entire sailing range of true wind speed and direction. Full-scale results will be used to verify the results of this section and to quantify how many linear models are really needed to fully characterize sailing yacht dynamics. 


\section{Experimental Set-up}

A full-scale yacht was outfitted with sensors to serve as an experimental platform for collecting full-scale system identification data. The test vehicle is the Precision $23 \mathrm{ft}$ daysailer Avanti, shown in Figure 20 and Figure 21. The length overall (LOA) of the Precision 23 is $23 \mathrm{ft}$, and it displaces 2,450 lbs. Avanti is an ideal platform to demonstrate the applicability of system identification techniques to a large range of monohull sailing yachts. It is very stable and large enough to not be as sensitive to crew weight and sail trim as, for example, a high performance racing yacht. Yet it is still small and lightweight enough to exhibit some of the more dynamic characteristics of either a racing yacht or a small dinghy. It may be sailed by one person, and has ample room to keep sensors and a powerful laptop dry and secure.

All tests were performed on Clinton Lake in Lawrence, KS. The lake environment is also very good for preliminary system identification as there is no water current to affect performance, and during a sail on the lake, the boat tends to experience frequent gusts and shifts, which will help characterize the dynamic response of the yacht to quick changes in wind. The

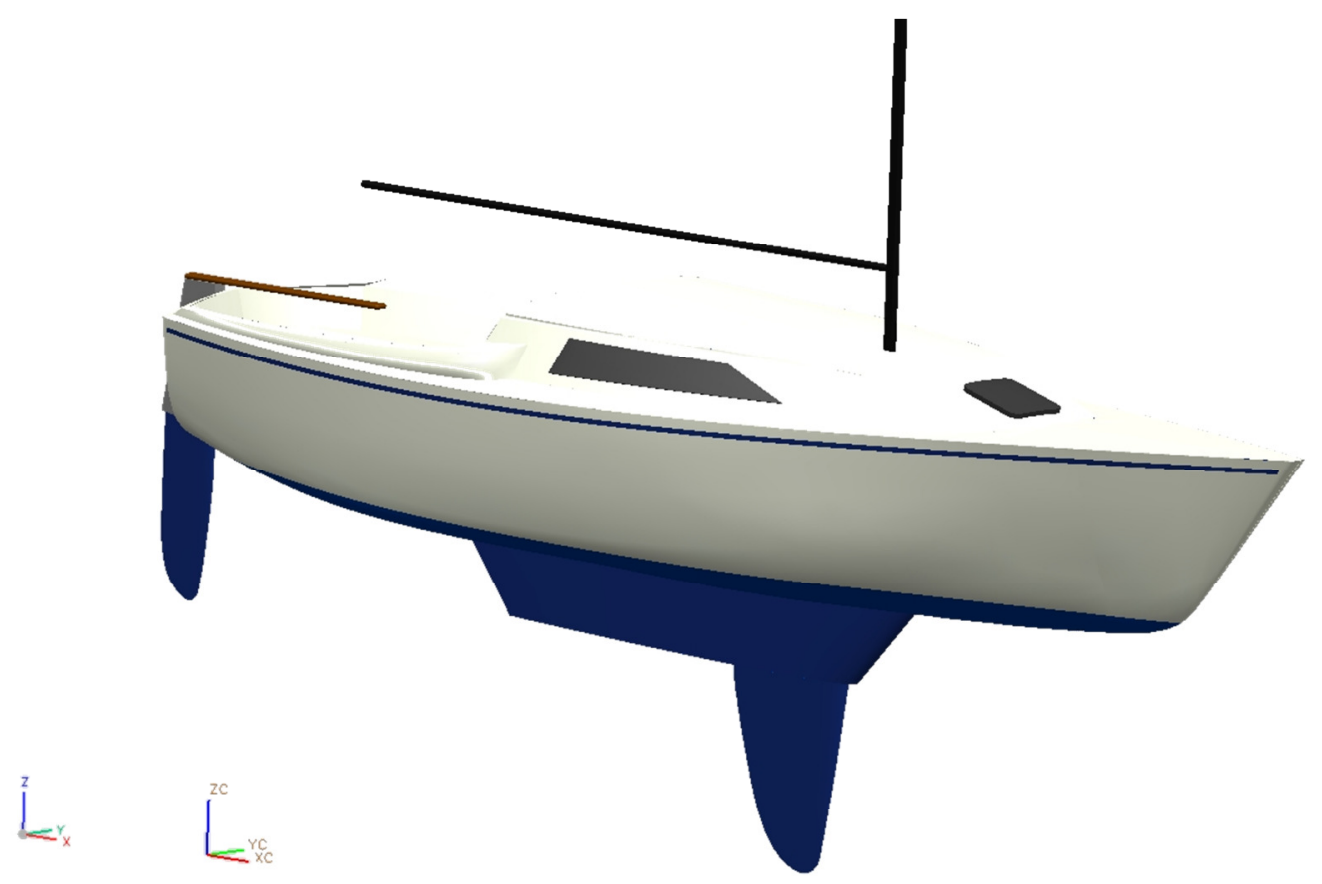

Figure 20: Precision 23 geometry reverse-engineered using photogrammetry to develop a computer-aided design (CAD) model. 
lake is sufficiently large to sail in a trimmed state for 5-10 minutes, providing the opportunity to repeat maneuvers several times while sailing in the same trim condition thus enabling the collection of data for both identification (ID data), and then subsequently for validation (TEST data).

\subsection{Data Acquisition System}

The type and quality of data recorded are extremely important to the success of estimation, thus direct measurements of the states, control inputs, and state derivatives are necessary. For the model presented, this includes measurements of the accelerations, $[\dot{U} \dot{V} \dot{P} \dot{R}]$, the body velocities, $\left[\begin{array}{llll}U & V & P & R\end{array}\right]$, the rudder angle $\delta_{r}$, the main sail deflection angle, $\delta_{b}$, the heel/roll angle $\phi$, and apparent wind speed and direction $\left[V_{A} \beta_{A}\right]$.

The sensor suite comprises three different electronic subsystems which feed data into an onboard laptop computer through the USB interface. The data is read, merged, and uniformly time stamped through a LabView virtual interface (VI). The first electronic subsystem is a standard NMEA 2000 (N2K) network with a paddlewheel speed transducer, ultrasonic masthead

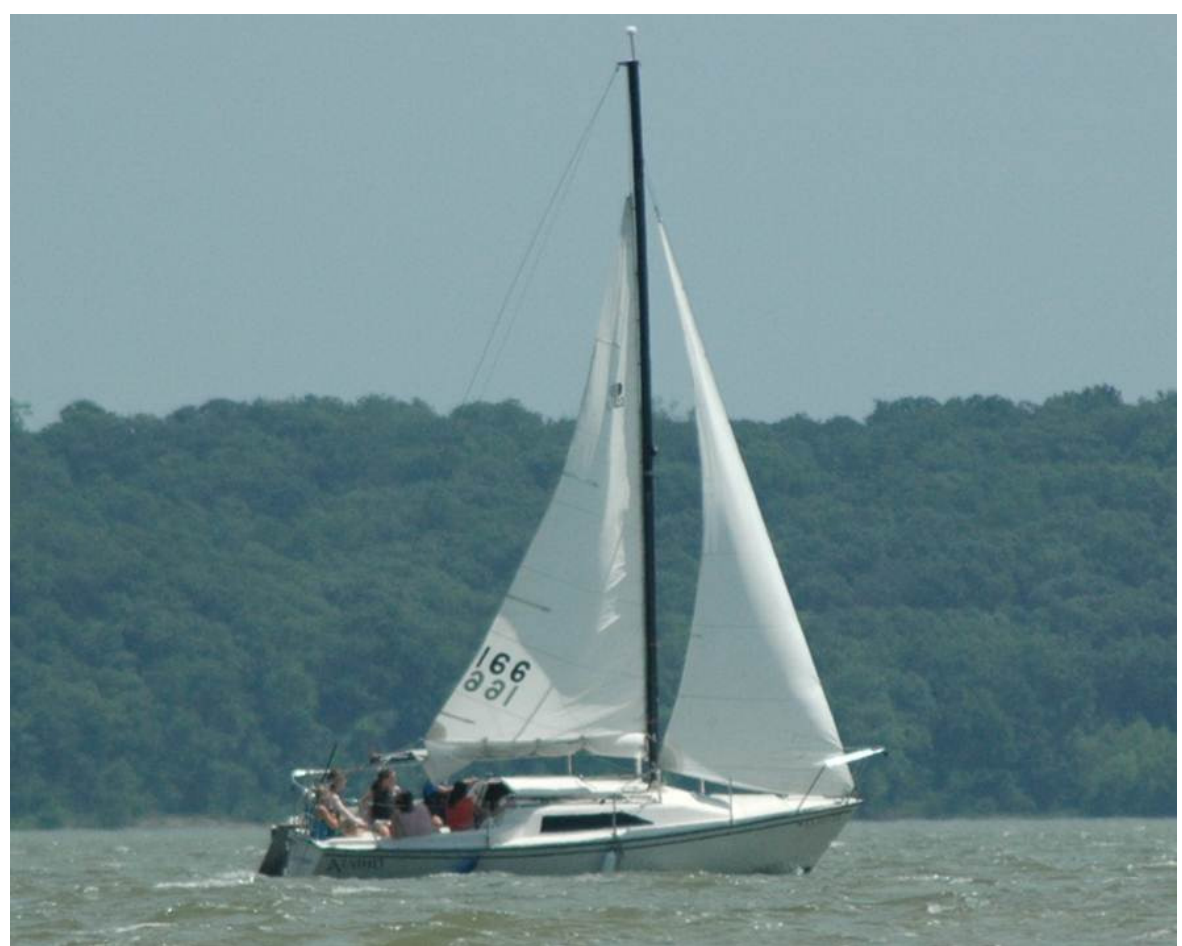

Figure 21: Avanti under sail on Clinton Lake in the Reefed Main and Jib sail configuration. 
weather station, GPS antenna, and rudder angle sensor. Use of the existing N2K network greatly simplified much of the design of the data acquisition system because of the wide range of rugged weatherproof sensors compatible with the network on the market. To send the information to the laptop, a Maretron USB100 translates the N2K messages to NMEA 0183 ASCII text format and then sends the data over a serial interface that is easily read by the VI. The masthead wind sensor is reported by the manufacturer to give accurate apparent wind speed and direction up to $30^{\circ}$ of heel.

The second subsystem is a NAV420 GPS/IMU/AHRS unit which provides linear accelerations, Euler angles $(\phi \psi \theta)$, and angular rates. This system uses a GPS signal and internal filters to keep the data produced by the accelerometers and gyros from drifting while in operation. The unit can provide either accelerometer data or velocities. For our system the accelerometer mode is utilized. The last part of the sensor suite is a National Instruments cDAQ9172 tray, which reads voltages from a potentiometer mounted on the boom. This tray has room for additional sensors, including extra accelerometers which have potential to increase the quality of the data. The three sets of streaming data are interpreted by a LabView VI file and uniformly time stamped into one data set. A data flow diagram is shown in Figure 22. The data was collected at $5 \mathrm{~Hz}$ and has been adequate to prove the methods in this work. It is possible for future work to collect data at a faster rate, up to $10 \mathrm{~Hz}$ for the current sensor suite, by redesigning

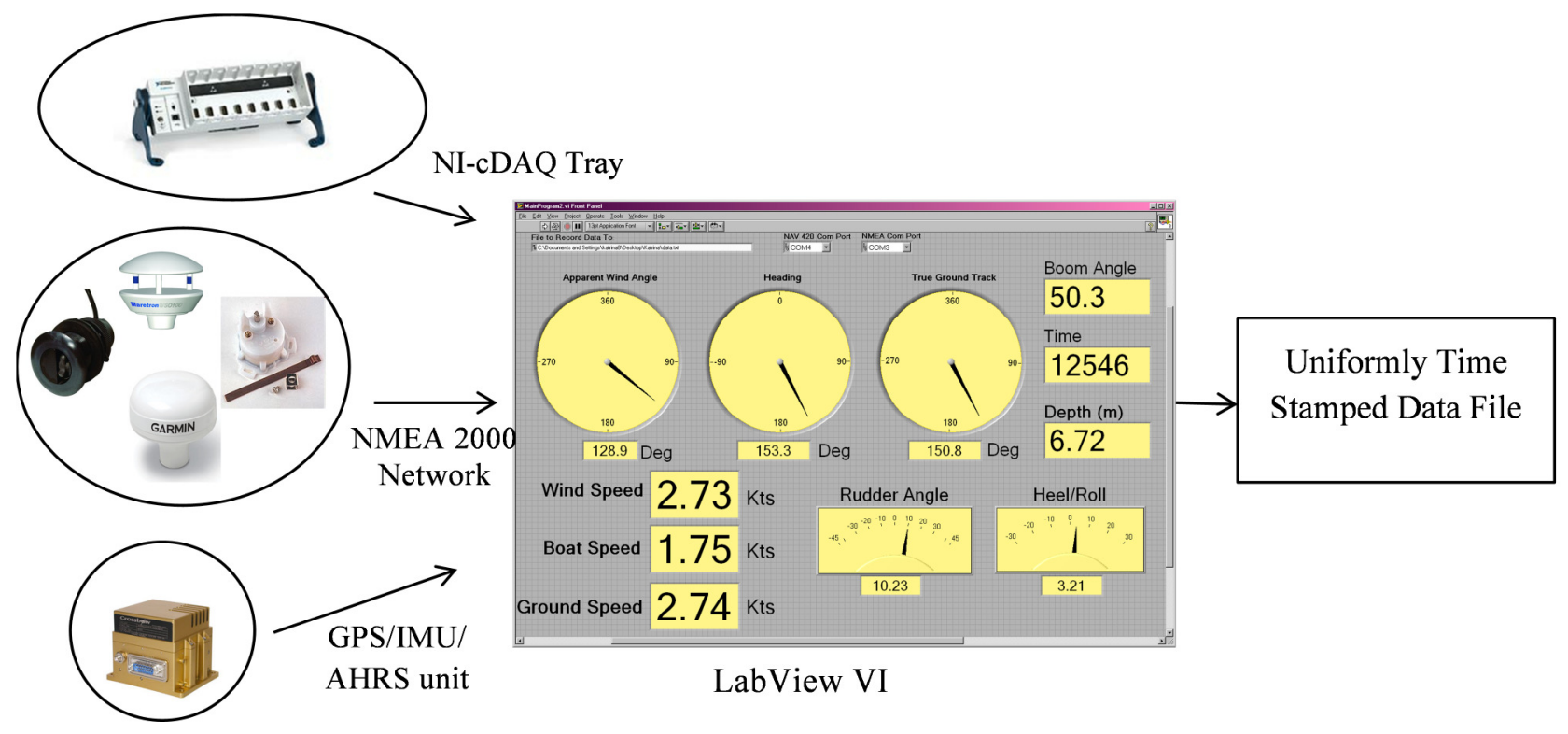

Figure 22: Data flow diagram of data acquisition system. 
the method by which the VI captures and reads the serial interface data.

\subsection{Testing Procedure}

The kind of data collected in terms of inputs is very important to the success of system identification. It is necessary to generate data that is rich enough in control input to excite all vehicle modes. In the case of the sailing yacht several parameters such as sail coefficients and hull drag are non-linear functions of state variables, so several experiments in varying wind angles and speeds must be performed to adequately characterize these variables to enable nonlinear system identification is performed. Also, performing tests in varying conditions gives a range of data for analyzing the effectiveness of the linear model at different trim points. The tests are focused on points of sail from close-hauled to a beam reach, examining the downwind condition is beyond the scope of this preliminary study, although a small amount of data has been collected in this condition for future analysis.

The test strategy is designed to generate sufficient data to not only perform system identification, but also to validate the resulting model by testing its ability to recreate measured data separate from that used for estimation. Each day, the boat is sailed at constant apparent wind angles on both port and starboard tacks ranging from close hauled (true wind $\sim 45^{\circ}$ ) to a beam reach (true wind $\sim 90^{\circ}$ ). While trimmed for a certain apparent wind angle, rudder inputs are given to the system in the form of singlets and doublets, shown in Figure 23. Singlets and doublets are meant to excite the dynamic oscillatory modes of the vehicle which will be particularly useful in evaluating the validity range of a linear yacht model. Maneuvers are performed by starting turns both to windward (into the wind) and to leeward (away from the wind) first. Experience on the water has shown the response characterized by the two cases is fairly different. The singlets and doublets are of varying amplitude and period because it is unknown a priori what input sequences will be sufficient to characterize the boat dynamics. 


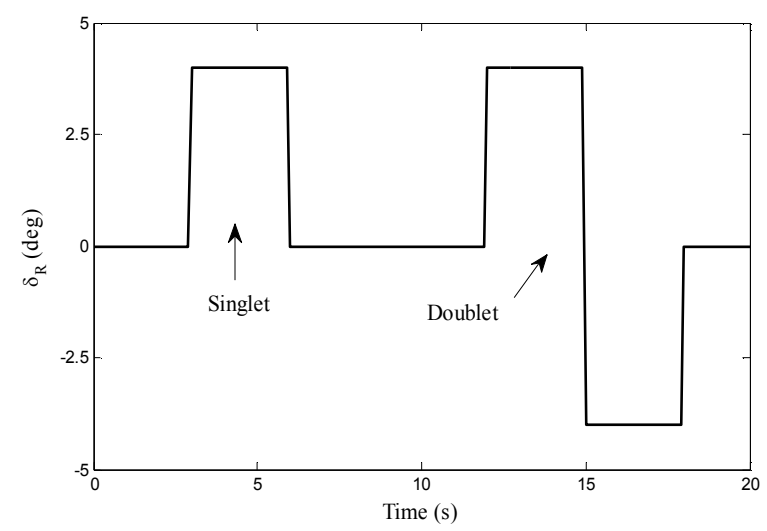

Figure 23: Sample rudder singlet and doublet.

The singlets and doublets are inputs designed specifically for identifying linear models. In addition to the singlets and doublets, the data recorded contains a great deal of trimmed sailing, tacking, and forcing the boat into irons (head to wind) after sailing an upwind course. These maneuvers may help estimate and validate the non-linear sailing yacht models in the future.

To characterize the effect the sail has on the yacht when it is sailing at a constant apparent wind angle, the sail is let out, effectively decreasing its angle of attack, until it luffs, then is brought back in to its original trim. This will be referred to as a boom singlet. In the upwind condition especially, this has large effects on the way the boat is sailing. In addition to characterizing the sail effect, wind gusts and shifts are also included in the data. When such an event occurs, the helmsman does not react to them, instead, the boat remains in the same configuration as before the gust or shift was experienced, and the boat is allowed to respond to the change on its own. It is very important to try and isolate these effects in the data and that as much as possible the boat is allowed to respond on its own without intervention by the helmsman or crew. This is to satisfy the assumption for methods, especially MLE, where the inputs are assumed to be independent of the system outputs.

\subsection{Summary of Data}

The majority of the data that will be used throughout this project was collected during fall of 2011. A total of about 20 hours of sailing data was collected over 7 days. During some of the days the weather changed enough that sail changes were required, so the data is truly representative of a variety of wind and weather conditions. On lighter wind days, data was collected with the full main sail and $150 \%$ genoa foresail, and this is labeled the Genoa 
configuration. When the boat became overpowered with this sail configuration, the foresail was switched to the $100 \% \mathrm{jib}$, and this is labeled the Jib configuration. During some of the days the main sail area was also reduced through reefing, and this is labeled the Reef configuration.

As will be shown in the following sub-sections, collecting full-scale data for system identification on a sailing yacht is a very challenging task. Conditions on the water a continually changing, and often it is difficult to isolate one change to the system long enough to record the response data. Also, when the data was initially collected, it was not possible to design 'optimal' inputs as is often done with aircraft because the dynamic behavior of a sailing yacht has not been studied in the past. Therefore many of the issues inherent in the data collection process did not surface until much of the data was post-processed and analysis completed. Despite the challenges, however, a suitable quantity of data was collected for use with linear system identification.

\subsubsection{Full Main and Genoa (Genoa Configuration)}

The Genoa configuration corresponds to wind speeds from zero to a maximum of $6 \mathrm{~m} / \mathrm{s}$ (13 $\mathrm{mph}$ ) for this boat. Because the genoa configuration represents overall low wind speeds, it also represents very small or in some cases zero roll angles. While it is certainly possible to sail the boat with the genoa at higher wind speeds and therefore higher heel angles, this was generally not the case when data was being collected. When deciding upon which sail configuration would be appropriate for a given testing day, if the wind speed was in the upper range in which the boat would carry the genoa, the conservative choice was made to use the smaller foresail, the jib. This decision was made mostly for safety reasons, as for a solo helmsman on the boat it is challenging to manage a sail change out on the water, especially when working under the time constraints of the laptop battery life of about two and one-half hours. Thus, the upper wind range which would correspond to higher roll angles is largely missing from the current set of data.

The second row represents the total maneuvers most suitable for the linear system identification. There are three main reasons a maneuver was not chosen from the linear system identification. The first is unique to the Genoa data, and that is the absence of a roll response at low wind speeds. The sail has primary control over the roll angle of the boat, which in turn is 

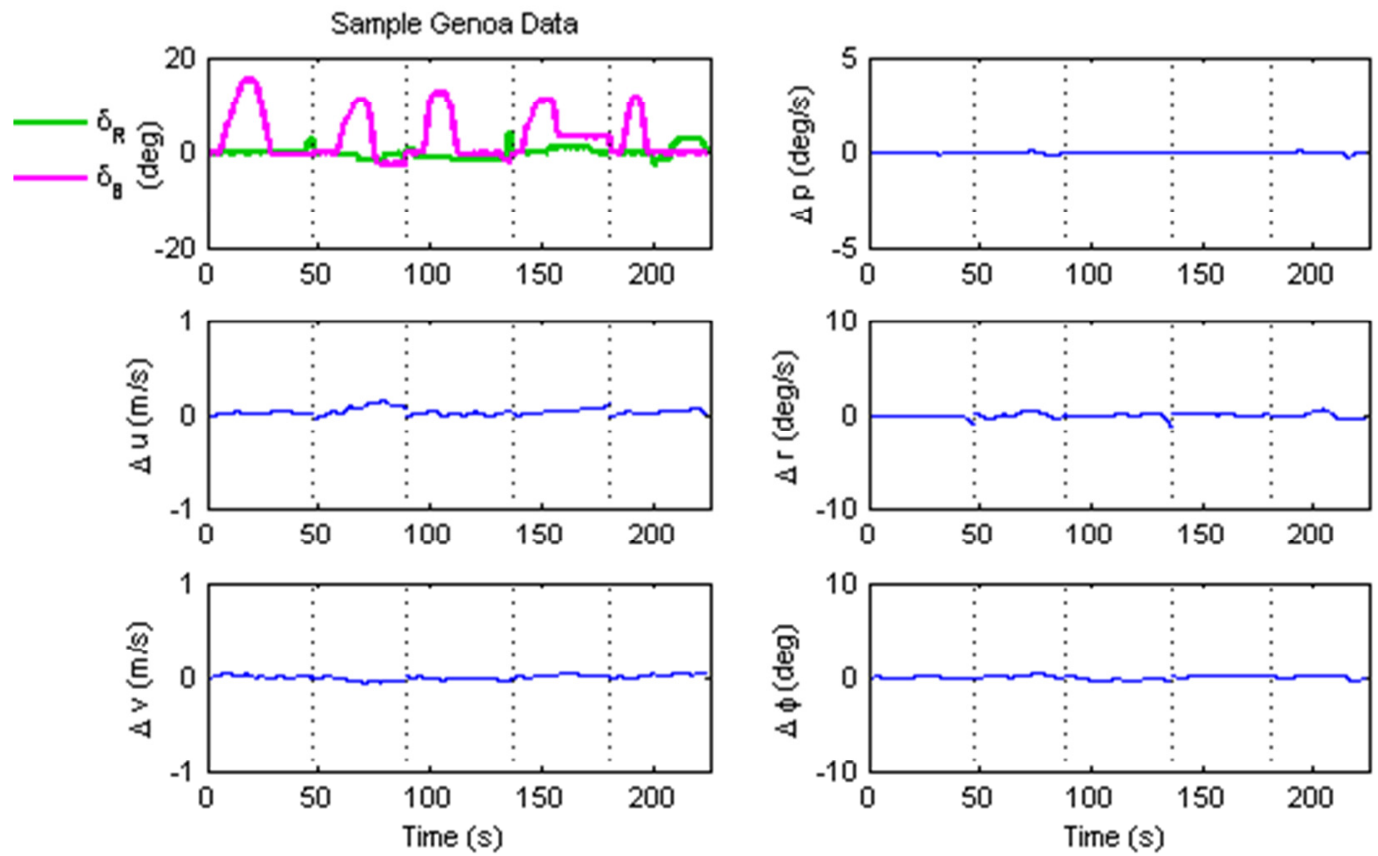

Figure 24: Example no roll response to boom singlets in a set of Genoa data
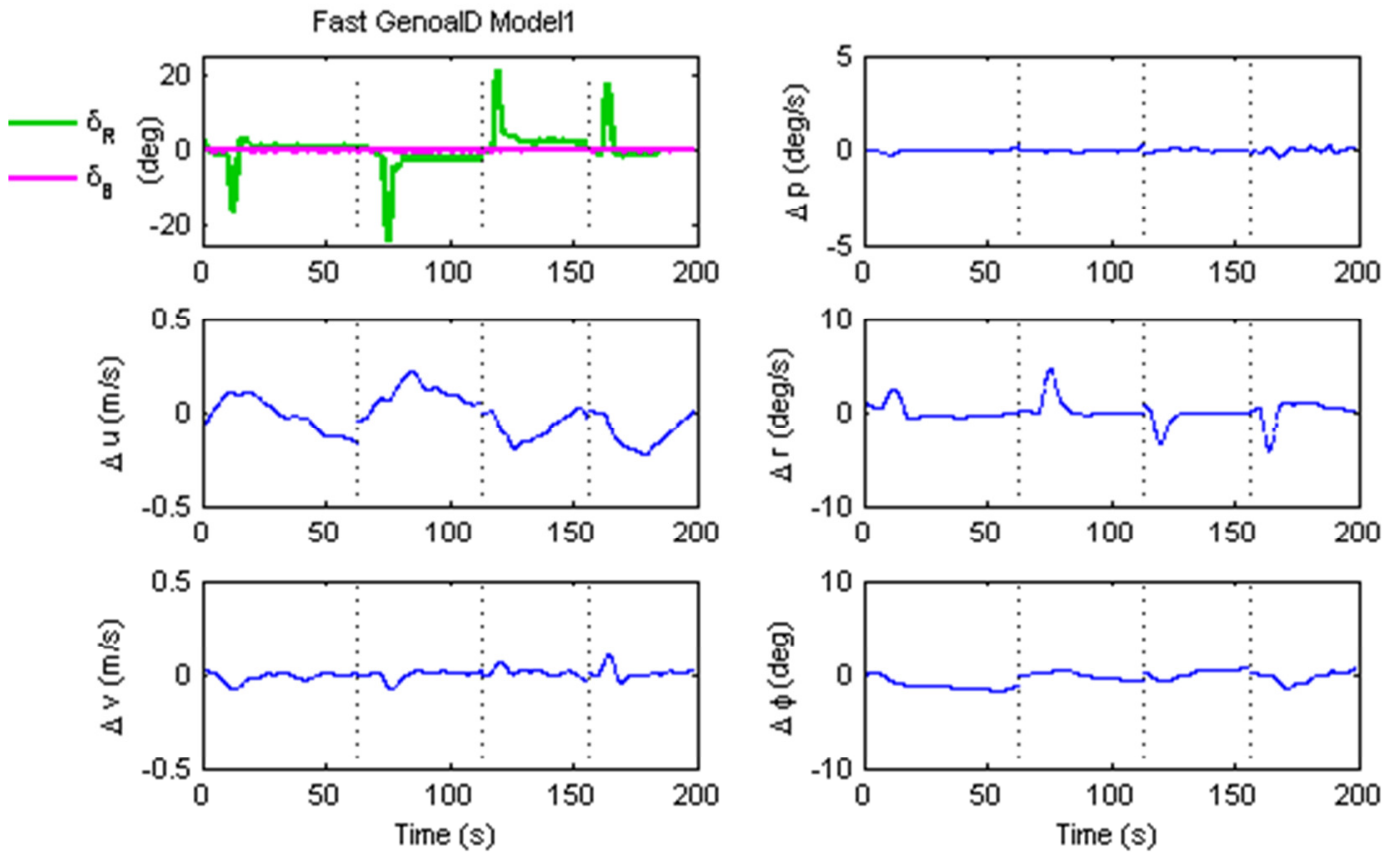

Figure 25: Example of a clear dynamic response in surge, sway, and yaw to rudder singlets, but no response in roll in a set of Genoa data.

dependent upon the wind. This is shown in Figure 24 where the response to several very consistent sail inputs is plotted. Figure 24 represents the case where there is not sufficient energy in the wind to actually roll the boat significantly, and it also shows that in this case the sail does 
not affect the other state variables. The data represented by Figure 24 is not useful for system identification because there is no dynamic response to measure or model. Figure 25 represents a step up from that by showing rudder inputs given to the boat in similar conditions. Here, there is a clearly a response in surge, sway, and yaw, but no response in roll. The data in Figure 25 is ideal for system identification; the response is very predictable and repeatable. However, the absence of the roll response means the linear model structure defined in Section 4.2 is not the appropriate model choice when the boat is sailing in these conditions. This will be further discussed in the section on linear system identification, Section 8.5.

The second reason such data would not be chosen for system identification is that not enough time was allowed during a test maneuver for the state variables, particularly surge, to fully recover their initial condition before either an external disturbance affected the system or another test maneuver was begun. There is a very simple solution to this problem, and that is to feed the surge velocity especially back to the skipper so sufficient time is allotted for the sailboat to recover naturally without a pilot control input.

Lastly, the third reason accounts for a fairly small portion of the overall maneuvers, but there were certainly cases where it was obvious that the test maneuver was not isolated, meaning too many variables on the boat were simultaneously changing. All three of the issues just discussed with the data collection are only important if the data is to be used for linear system identification. It should be stressed most of this data could be used for non-linear system identification, especially with a technique like neural networks.

\subsubsection{Full Main and Jib (Jib Configuration)}

The largest amount of data was collected in this configuration, representing wind speeds ranging from $5 \mathrm{~m} / \mathrm{s}$ to $8 \mathrm{~m} / \mathrm{s}$ (11 to $18 \mathrm{mph}$ ). With the wind up, the data in this configuration is rich in dynamic response, with a wide range of trimmed wind speeds and heel angles

Table 3: Summary of maneuvers collected in the Genoa configuration.

\begin{tabular}{|c|c|c|c|}
\hline & $\begin{array}{c}\text { Rudder } \\
\text { Singlets }\end{array}$ & $\begin{array}{c}\text { Rudder } \\
\text { Doublets }\end{array}$ & Boom Singlets \\
\hline All Collected Maneuvers & 65 & 37 & 23 \\
Suitable Maneuvers for Linear System ID & 13 & 5 & 9 \\
\hline
\end{tabular}


Table 4: Summary of maneuvers collected in the Jib configuration.

\begin{tabular}{|c|c|c|c|}
\hline & $\begin{array}{c}\text { Rudder } \\
\text { Singlets }\end{array}$ & $\begin{array}{c}\text { Rudder } \\
\text { Doublets }\end{array}$ & Boom Singlets \\
\hline All Collected Maneuvers & 69 & 28 & 36 \\
Suitable Maneuvers for Linear System ID & 35 & 9 & 21 \\
\hline
\end{tabular}

represented. There is still a significant loss, however, from the total collected maneuvers to the suitable maneuvers for system identification as shown in Table 4. There were ample maneuvers to choose from in this configuration, and only the best maneuvers were chosen for linear system identification. In this condition the issue of not allowing the surge velocity enough time to recover was the primary reason not to use a maneuver.

\subsubsection{Reefed Main and Jib (Reef Configuration)}

The least amount of usable data was collected in this configuration. This is a very interesting wind range for the boat as it represents the limits of what it can handle thus one would expect significantly non-linear behavior. The Reef configuration corresponds with the highest wind speeds, and most challenging sailing conditions, especially for a solo sailor. Unfortunately, much data was lost because the laptop was not visible from the cockpit, and in these conditions there were several times where hitting a wave was just enough force to disconnect one of the electronic sensors which in turn caused the data acquisition system to stop recording data. The electrical connection problem could certainly have been addressed in the Labview VI, but the problem was realized after the fact. Lastly, this was the most difficult configuration in which to isolate the maneuvers and responses for system id because on the lake environment the conditions were rapidly changing and there was large chop wave action and significant wind gusting. The specific number of maneuvers performed with the reefed main sail configuration was not recorded on the water because of the difficulty in sailing single-handed in heavy wind and wave conditions. Thus in the first post-processing stage only maneuvers that were suitable

Table 5: Summary of suitable maneuvers collected in the Reef configuration

\begin{tabular}{|l|c|c|c|}
\hline & $\begin{array}{c}\text { Rudder } \\
\text { Singlets }\end{array}$ & $\begin{array}{c}\text { Rudder } \\
\text { Doublets }\end{array}$ & Boom Singlets \\
\hline Suitable Maneuvers for Linear System ID & 2 & 4 & 2 \\
\hline
\end{tabular}


for system identification were extracted from the full scale data. The total number of maneuvers is shown in Table 5.

In summary, this chapter has presented a full-scale sailing yacht test platform. The data captures the motion variables of the test platform in three sail configurations. This data will be analyzed in subsequent chapters. 


\section{System Identification}

\subsection{Overview}

System identification techniques are approaches to modeling where the defining model is extracted from real data. A subset of system identification is parameter estimation, which is when the appropriate model structure is known except for a defining set of constant parameters that make the system unique. For a sailing yacht, this would be a non-linear dynamic model like that presented in Section 3.5. These methods minimize errors between time history predicted by the assumed model and actual measured time history. Some common estimation methods include least squares, maximum likelihood, and artificial neural networks. The first two methods mentioned assume a priori knowledge of the system to be identified; neural networks do not. Identified models can be assessed by their ability to independently reproduce measured data.

Equation error methods include least squares (LS) and are regression techniques that minimize a cost function which is defined in terms of an input/output equation. For linearized systems, it is possible to compute the unknown system parameters, such as force coefficients, in one step. For non-linear systems such as the sailing yacht, equation error methods can be used by artificially transforming the system into a linear least square problem and solved in one step or solved iteratively. Eq. (3.10) shows the hydrodynamic force model is nonlinear in the state but linear in the parameter. For models containing this sort of nonlinearity, it is possible to precompute the nonlinear terms, such as $V^{\prime 2}$ and $\phi^{2}$, and append them to the model as artificial states and still use a one shot LS approach (Billings, 1980; Jategaonkar, 2006; Ljung, 1999). Eq. (3.18) demonstrate nonlinearity in the parameter. In these equations, the parameters like $X_{\varepsilon}$ are contained within a sin function. In this case, LS can still be utilized, but only in an iterative fashion because the cost function cannot be minimized in one step (Jategaonkar, 2006). One limitation of equation error methods is the presence of substantial measurement error, a problem that can be alleviated with high quality sensors and instrumentation, as well as data preprocessing. 
Output error methods include maximum likelihood estimation (MLE) and are based on probability theory. MLE handles both process and measurement errors because this method treats the measurements themselves as unreliable (Ljung, 1999). The measurement noise may come from instrument biases, scale factors, misalignments, and random errors. Preprocessing data to remove bias and scale factoring problems is common, leaving the random noise to be dealt with by the estimation algorithm.

The main difference between MLE and LS is the cost function, for MLE it is the statistically derived likelihood function, for

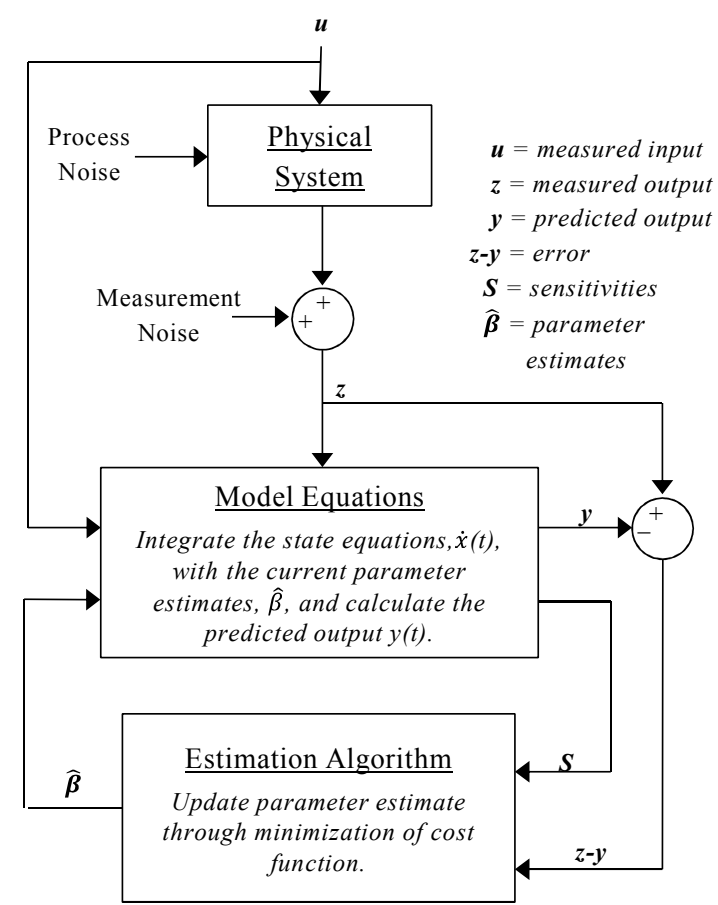

Figure 26: Diagram of MLE and iterative LS estimation structure

LS it is simply the sum square difference between the measured output and the predicted output. Algorithmically, MLE and iterative LS are implemented in a very similar way shown in Figure 26. Parameter estimates are generated through the minimization of the cost function by an optimization method such as Gauss-Newton or Levenburg-Marquardt.

For parameters like $X_{S}^{\prime}$ and $X_{0}$ which are nonlinear functions of $\beta_{A}$ and $U$, respectively, it is possible to use equation and output error methods to generate appropriate estimates at preassigned 'knot' locations (Paris \& Bonner, 2004), essentially generating a look-up table for those parameters. A knot location for $X_{0}$ would be a velocity, $U$, and for $X_{S}^{\prime}$ a specific apparent wind angle. The value of the parameter can then be interpolated between the known locations, generating a look-up table for the parameters. As previously mentioned, multiple experiments at different knot locations (different apparent wind angles and boat speeds) must be performed to generate this data.

Neural networks are different as they employ a numerical search rather than a minimization procedure to develop what is called a "black box" model. Such a model has essentially no 
physical interpretation, but generally produces models that, given a set of measured inputs (wind speed, direction, sail settings) will accurately predict measured system outputs (speed and heading). This technique is the only estimation technique that has been applied to sailing yachts (Masuyama et al., 1995; Tiano, Zirilli, Yang, \& Xiao, 2001) however it was not used to estimate parameters, instead it was used to simulate the system. The advantage of neural networks is they do not require a model structure, initial parameter guesses, or a stable system to perform system identification.

Although it is a "black box" technique not based on physical system equations, neural networks have been successfully used to extract physical aircraft parameters using measured flight data (Amin, Gerhart, \& Rodin, 1997; Das, Kuttieri, Sinha, \& Jategaonkar, 2010; Linse \& Stengel, 1993; Peyada \& Ghosh, 2009; Raisinghani, Bhaskar Reddy, \& Ghosh, 1999). This is done through what is called the 'delta' method, which pre-trains a neural network to measured inputs and outputs, then uses the resulting network to simulate the vehicle varying one input at a time and thus obtaining parameter estimates. This approach lends itself very well to application to a sailing yacht because of its ability to capture complex non-linear system behavior.

Three methods of system identification have been presented. Of the three, LS techniques are the most straightforward to implement and have been chosen as the methods of choice for this dissertation. Neural networks provide a different yet advantageous approach to estimation which doesn't require the physical model for estimation. While not chosen for this work, they may offer a good solution for non-linear sailing yacht model identification in the future.

\subsection{One Shot Least Squares Formulation}

The LS algorithm may be solved in one step for linear systems, thus, computationally it goes very fast and can be applied to several large sets of data and yield estimated models very quickly. This methodology is applied to the full-scale data to obtain an understanding of the system dynamics, what kind of modes to expect, and to provide insight as to whether estimated linear models may be suitable for future control system design.

The one step LS estimator for the 4DOF state space model with 2 controls is formulated as follows. The state space model is of the form: 


$$
\dot{\bar{x}}(t)=A \vec{x}(t)+B \vec{u}(t)
$$

Where the state vector will be defined as:

$$
\vec{x}=\left[\begin{array}{lllll}
\Delta u & \Delta v & \Delta p & \Delta r & \Delta \phi
\end{array}\right]^{T}
$$

The control vector will be defined as:

$$
\vec{u}=\left[\begin{array}{ll}
\Delta \delta_{r} & \Delta \delta_{b}
\end{array}\right]^{T}
$$

The measurement vector will be defined as:

$$
X_{m}=\left[\begin{array}{lllll}
\Delta u_{m} & \Delta v_{m} & \Delta p_{m} & \Delta r_{m} & \Delta \phi_{m}
\end{array}\right]^{T}
$$

The unknown parameter matrix is formed by combining the $A$ and $B$ matrices into a single partitioned matrix, and may be solved for using the formula in Eq. (6.6) where $N$ is the number of measurements (Jategaonkar, 2006).

$$
\begin{gathered}
\bar{\beta}=\left[\begin{array}{cc}
\mathbf{I} & A \\
B & \mathbf{0}
\end{array}\right] \\
\bar{\beta}^{T}=\left\{\sum_{k=1}^{N}\left[\begin{array}{c}
\vec{u} \\
X
\end{array}\right]\left[\begin{array}{c}
\vec{u} \\
X_{m}
\end{array}\right]^{T}\right\}^{-1}\left\{\sum_{k=1}^{N}\left[\begin{array}{c}
\vec{u} \\
X_{m}
\end{array}\right]\left[\begin{array}{c}
\vec{u} \\
\dot{X}_{m}
\end{array}\right]^{T}\right\}
\end{gathered}
$$

\subsection{Maximum Likelihood Formulation}

The maximum likelihood cost function for parameter estimation in dynamic systems is:

$$
\begin{aligned}
J(\vec{\beta}, R)= & \frac{1}{2} \sum_{k=1}^{N}\left[\vec{y}\left(t_{k}\right)-\hat{\bar{y}}\left(t_{k}\right)\right]^{T} R^{-1}\left[\vec{y}\left(t_{k}\right)-\hat{\bar{y}}\left(t_{k}\right)\right] \ldots \\
& +\frac{N}{2} \ln (\operatorname{det}(R))+\frac{N n_{y}}{2} \ln (2 \pi)
\end{aligned}
$$

Where $\vec{\beta}$ is the vector of unknown parameters, $n_{y}$ is the number of outputs, $\hat{\bar{y}}$ is the vector of estimated system outputs, and $\bar{y}$ is the vector of measured system outputs. Measurements are made at $N$ discrete times $t_{k}$. $R$ is the measurement noise covariance matrix, and in the case of our system is assumed to be unknown. If a value of the parameter vector $\vec{\beta}$ is assumed, then the maximum likelihood estimate for $R$ is: 


$$
R=\frac{1}{N} \sum_{k=1}^{N}\left[\vec{y}\left(t_{k}\right)-\hat{\bar{y}}\left(t_{k}\right)\right]\left[\vec{y}\left(t_{k}\right)-\hat{\bar{y}}\left(t_{k}\right)\right]^{T}
$$

Plugging the estimate in for $R$ in the cost function of Eq. (6.8) results in the following expression:

$$
J(\vec{\beta})=\frac{N n_{y}}{2}+\frac{N}{2} \ln (\operatorname{det}(R))+\frac{N n_{y}}{2} \ln (2 \pi)
$$

For any given model and set of estimation data, the numbers $N$ and $n_{y}$ will be specified, therefore, the first and last terms of Eq. (6.9) are constant and may be neglected for the minimization of the cost function. Therefore, the cost function reduces to:

$$
J(\vec{\beta})=\operatorname{det}(R)
$$

The parameter estimation problem then becomes to determine the appropriate parameter vector $\vec{\beta}$ that will satisfy the minimization of the determinant of $R$. This may be performed by an optimization procedure which determines how to change the original parameter estimates $\vec{\beta}$ in a way which optimally decreases the value of the cost function. The process goes as follows:

1) Formulate a reasonable guess as to the value of the parameters in $\vec{\beta}$.

2) Use the parameter guess to calculate the system outputs, $\hat{\bar{y}}$. For the purposes of the nonlinear sailing yacht dynamic model, this means simulating the system defined in Eqs. (3.2) - (3.5) with the measured inputs of the sails and rudder. For system identification, these inputs are considered exogenous, meaning the input sequence is generated independent of the system output. This means this process assumes there is no feedback and the control inputs are independent of the output.

3) With the estimated model output, $\hat{\bar{y}}$, calculated, it is then possible to compute the residuals $(\vec{y}-\hat{\bar{y}})$.

4) Minimize $J(\vec{\beta})$ with respect to $\vec{\beta}$ with an optimization technique.

5) Iterate on steps 2 - 4 until convergence is achieved between the estimated model output and the measured model output. 
In order to calculate the cost function, first an appropriate value of the parameters in $\vec{\beta}$ is guessed, then the values in $\hat{\bar{y}}$ are calculated based upon that parameter estimate. This allows real computation of the cost function in Eq. (6.7). The minimization procedure is usually an optimization procedure which determines how to change the original parameter estimates $\vec{\beta}$ in a way which optimally decreases the value of the cost function. .

\subsubsection{Minimization of the cost function}

In order to minimize the cost function, the partial derivative is taken with respect to the unknown parameters and set to zero, thereby establishing the condition for a minimum:

$$
\frac{\partial J(\vec{\beta})}{\partial \vec{\beta}}=0
$$

The change in parameters from the current step, $i$, to the next step, $i+l$ is defined as:

$$
\vec{\beta}_{i+1}=\vec{\beta}_{i}+\Delta \vec{\beta}
$$

Where $\Delta \vec{\beta}$ represents an incremental change in the original parameters guess. In order for the next guess to be as close as possible to the solution the derivative of the cost function presented in Eq. (6.11) is expanded in a Taylor series containing the value of $\Delta \vec{\beta}$ :

$$
\frac{\partial J}{\partial \vec{\beta}} \approx\left(\frac{\partial J}{\partial \vec{\beta}}\right)_{i}+\left(\frac{\partial^{2} J}{\partial \vec{\beta}^{2}}\right)_{i} \Delta \vec{\beta}
$$

Note that $\vec{\beta}$ has been dropped from the $J$ function from here on to simplify the notation. Eq. (6.13) may be re-arranged to solve for $\Delta \vec{\beta}$ :

$$
\Delta \vec{\beta}=\left(\frac{\partial^{2} J}{\partial \bar{\beta}^{2}}\right)_{i}^{-1}\left(\frac{\partial J}{\partial \bar{\beta}}\right)_{i}
$$

Now the terms in Eq. (6.14) must be derived in terms of the model output equation $\hat{\bar{y}}\left(t_{k}\right)$ which is defined by the physical non-linear model. There are several methods of approaching the problem of calculating these terms, and a complete treatment of the subject is beyond the scope of this work. The method chosen here is that of quasi-linearization which is closely related to the model linearization presented in Chapter 4, but instead of linearizing about a nominal trim 
condition, the linearization is performed about a nominal vector of parameters, $\vec{\beta}_{0}$. First, the analytical expressions for the terms in Eq. (6.14) may be written as:

$$
\begin{gathered}
\frac{\partial J}{\partial \vec{\beta}}=-\sum_{k=1}^{N}\left[\frac{\partial \hat{\bar{y}}\left(t_{k}\right)}{\partial \vec{\beta}}\right]^{T} R^{-1}\left(\vec{y}\left(t_{k}\right)-\hat{\bar{y}}\left(t_{k}\right)\right)=0 \\
\frac{\partial^{2} J}{\partial \vec{\beta}^{2}}=-\sum_{k=1}^{N}\left[\frac{\partial \hat{\bar{y}}\left(t_{k}\right)}{\partial \vec{\beta}}\right]^{T} R^{-1} \frac{\partial \hat{\bar{y}}\left(t_{k}\right)}{\partial \vec{\beta}}+\sum_{k=1}^{N}\left[\frac{\partial^{2} \hat{\bar{y}}\left(t_{k}\right)}{\partial \vec{\beta}^{2}}\right]^{T} R^{-1}\left(\vec{y}_{i}\left(t_{k}\right)-\hat{\bar{y}}_{i}\left(t_{k}\right)\right)
\end{gathered}
$$

The linearization about the nominal $\vec{\beta}_{0}$ may be written as:

$$
\hat{\bar{y}}\left(t_{k}, \vec{\beta}\right) \approx \hat{\bar{y}}\left(t_{k}, \vec{\beta}_{0}\right)+\frac{\partial \hat{\bar{y}}\left(t_{k}\right)}{\partial \vec{\beta}} \Delta \vec{\beta}
$$

Inserting Eq. (6.17) into Eq. (6.15):

$$
-\sum_{k=1}^{N}\left[\frac{\partial \hat{\bar{y}}\left(t_{k}\right)}{\partial \vec{\beta}}\right]^{T} R^{-1}\left(\vec{y}_{i}\left(t_{k}\right)-\left\{\hat{\bar{y}}\left(t_{k}, \vec{\beta}_{0}\right)+\frac{\partial \hat{\bar{y}}\left(t_{k}\right)}{\partial \vec{\beta}} \Delta \vec{\beta}\right\}\right)=0
$$

Which when rearranged leads to a system of linear equations:

$$
\underbrace{\sum_{k=1}^{N}\left[\frac{\partial \hat{\bar{y}}\left(t_{k}\right)}{\partial \vec{\beta}}\right]^{T} R^{-1}\left[\frac{\partial \hat{\bar{y}}\left(t_{k}\right)}{\partial \vec{\beta}}\right]}_{\mathcal{F}} \Delta \vec{\beta}=\underbrace{\sum_{k=1}^{N}\left[\frac{\partial \hat{\bar{y}}\left(t_{k}\right)}{\partial \vec{\beta}}\right]^{T} R^{-1}\left(\vec{y}\left(t_{k}\right)-\hat{\bar{y}}\left(t_{k}, \vec{\beta}_{0}\right)\right)}_{-\mathcal{G}}
$$

Note that now everything is in terms of the first order derivative. The second order partial derivative has become the matrix of second gradients, $\mathcal{F}$, termed the Hessian matrix. The right hand side of Eq. (6.19) is termed the gradient vector, $\mathcal{G}$. We now have a simplified form with which to calculate the parameter perturbation by matrix inversion:

$$
\mathcal{F} \Delta \vec{\beta}=-\mathcal{G}
$$

The Hessian matrix, $\mathcal{F}$, is symmetric positive definite, thus Cholesky factorization may be used to compute the inversion in Eq. (6.20). With the perturbation $\Delta \vec{\beta}$, the parameter vector can be updated using Eq. (6.12). The Levenberg-Marquardt method modifies Eq. (6.20) with a parameter $\lambda$ which can be varied to control the direction of search for $\Delta \vec{\beta}$. Details on the 
Levenberg-Marquardt method may be found in (Jategaonkar, 2006) and (Bevington \& Robinson, 2003).

\subsection{Model Validation}

In the case of models estimated from the full-scale sailing data, there are no previous models or data with which to evaluate the result. Thus, estimated models will be judged by their predictive capabilities using both the data used for estimation (ID data) as well as separate data not used for estimation (TEST data). Models will be evaluated in simulation where the model's response to measured inputs is simulated then compared to actual measurements of the response. In the case of a linear model, only the first five seconds of the open loop simulation results may be used due to the compounding of errors inherent in the assumption of a linear model. The two measures of model fidelity that will be used are the coefficient of determination, $R^{2}$, and Theil's Inequality coefficient, $U_{T}$, both calculated using the simulated model response and the measured response.

Both of the evaluation methods are rooted in statistical theory, and make use of the definitions of standard deviation and covariance. In the case of evaluating the response of an estimated model, measurements are made for each of the number of states, $i$, for the number of time steps, $k$. For each of the measured data points, $y_{i}\left(t_{k}\right)$, there will be a corresponding data point, $\hat{y}\left(t_{k}\right)$, which is the response calculated by the estimated model from the same set of measured inputs. The standard deviation of each state measurement signal is given in Eq. (6.21) and of each state estimated signal is given in Eq.(6.22).

$$
\begin{array}{cc}
\sigma_{y_{i}}=\sqrt{\frac{1}{N} \sum_{k=1}^{N}\left[y_{i}\left(t_{k}\right)-\bar{y}_{i}\right]^{2}} \quad i=1,2, \ldots N x \\
\sigma_{\hat{y}_{i}}=\sqrt{\frac{1}{N} \sum_{k=1}^{N}\left[\hat{y}_{i}\left(t_{k}\right)-\hat{\bar{y}}_{i}\right]^{2}} \quad i=1,2, \ldots N x
\end{array}
$$

Where $\bar{y}_{i}$ and $\hat{\bar{y}}_{i}$ are the means of each of the state signals given in Eq. (6.23).

$$
\bar{y}_{i}=\frac{1}{N} \sum_{k=1}^{N} y_{i}\left(t_{k}\right) \quad \hat{\bar{y}}_{i}=\frac{1}{N} \sum_{k=1}^{N} \hat{y}_{i}\left(t_{k}\right) \quad i=1,2, \ldots N x
$$

The covariance of the two signals is given in Eq. (6.24). 


$$
\sigma_{y_{i} \hat{y}_{i}}=\frac{1}{N} \sum_{k=1}^{N}\left(y_{i}\left(t_{k}\right)-\bar{y}_{i}\left(t_{k}\right)\right)\left(\hat{y}_{i}\left(t_{k}\right)-\hat{\bar{y}}_{i}\left(t_{k}\right)\right) i=1,2, \ldots N x
$$

Armed with these basic statistical definitions, it is possible to define the coefficient of determination, $R^{2}$, as the square of the Pearson product moment correlation coefficient, $r$, which is a measure of the linear correlation of the set of points $\left(\vec{y}_{i}, \hat{\bar{y}}_{i}\right)$. The formula for calculating $R^{2}$, given in Eq. (6.26), is the ratio of the covariance of the estimated and measured signal divided by the product of their standard deviations. Thus, it represents a measure the linear correlation of the estimated and measured model responses.

$$
\begin{gathered}
r_{i}=\frac{\sigma_{y_{i} \hat{y}_{i}}}{\sigma_{y_{i}} \sigma_{\hat{y}_{i}}} i=1,2, \ldots N x \\
R_{i}^{2}=\left[\frac{\sigma_{y_{i} \hat{y}_{i}}}{\sigma_{y_{i}} \sigma_{\hat{y}_{i}}}\right]^{2} i=1,2, \ldots N x
\end{gathered}
$$

In the case of a perfect model fit, i.e. $y_{i}\left(t_{k}\right)=\hat{y}_{i}\left(t_{k}\right)$ for all $k$, then $R^{2}$ will be 1 , corresponding to an indication that all the points $\left(y_{i}\left(t_{k}\right), \hat{y}_{i}\left(t_{k}\right)\right)$ lie on a straight line, and may be said to be linearly correlated. In other words, $R^{2}$ of 1 indicates the points $\left(y_{i}\left(t_{k}\right), \hat{y}_{i}\left(t_{k}\right)\right)$ lie perfectly on the line defined by Eq. (6.27) where alpha and beta are arbitrary constants (Taylor, 1997).

$$
\hat{y}_{i}\left(t_{k}\right)=\alpha+\beta y_{i}\left(t_{k}\right)
$$

Graphically, this is shown in Figure 27(a). The scatter plot is the plot of the set of points $\left(\vec{y}_{i}, \hat{\bar{y}}_{i}\right)$, and the signal time history is the actual measured (in blue) and simulated (in orange) time history of the signal. Note that the 'discontinuities' in the time history are places at which the simulation is restarted with a different set of measurements and initial conditions, representing different segments of data. In the cases with no correlation, meaning the points $\left(\vec{y}_{i}, \hat{\bar{y}}_{i}\right)$ do not tend to lie on a straight line, as shown in Figure 27(b), $R^{2}$ will be close to zero. In cases with an infinite number of points, it would be exactly zero, but this is never possible with a finite number of points as represented by the data. Thus, $R^{2}$ is bounded between zero and one (Taylor, 1997). 
(a)

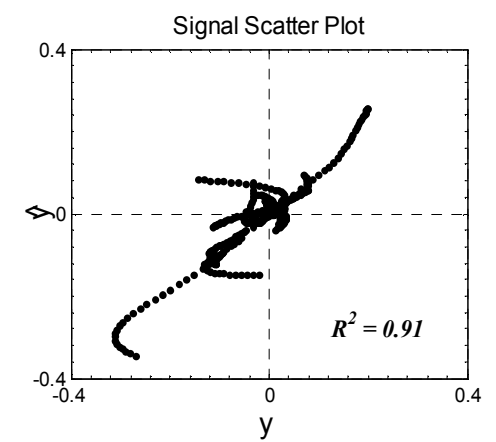

(b)

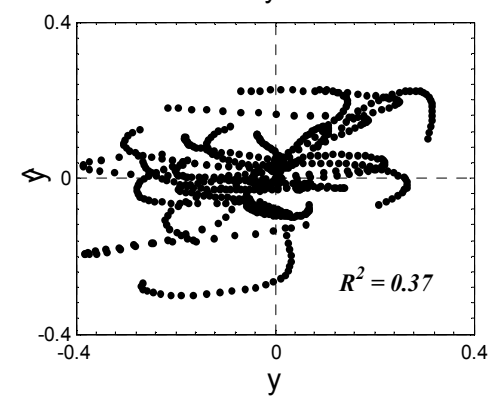

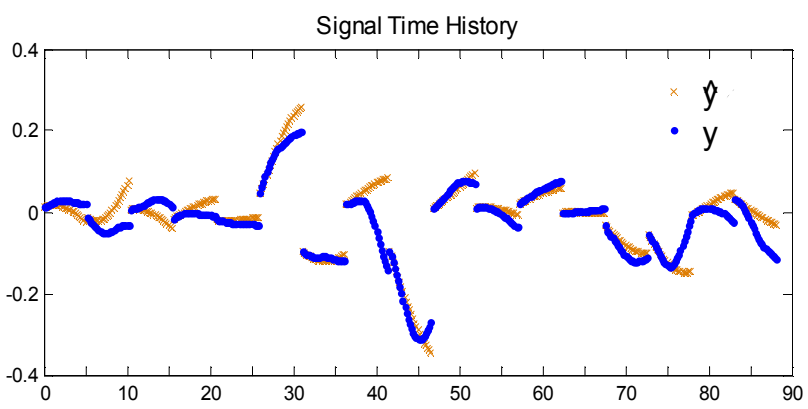

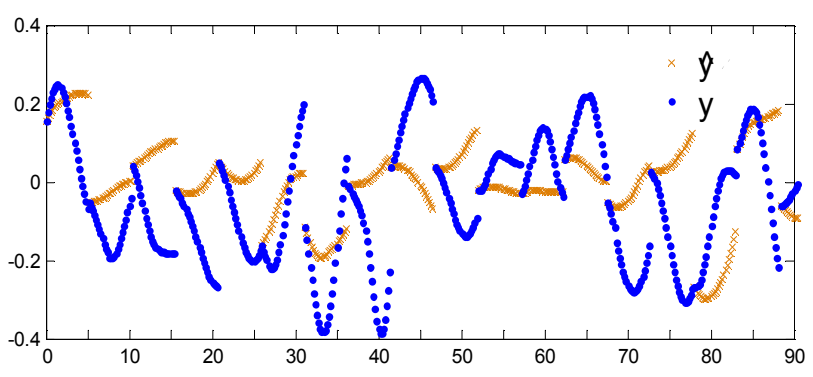

Figure 27: (a) Example of highly correlated model fit with $R^{2}$ of 0.91 (b) Example of poorly correlated model fit with $\boldsymbol{R}^{2}$ of 0.367

So far only the $R^{2}$ for the individual states has been presented. In order to get an overall single value for $R^{2}$ for an entire model the data in each state is normalized using the maximum value of the absolute value of the measured state vector. The normalized data is calculated as shown in Eqs. (6.28) and (6.29). Thus, all values for $\vec{y}_{i}$ will be bounded between -1 and 1 . This is not necessarily true for $\hat{\bar{y}}_{i}$, as it has been normalized according to the maximum from $\vec{y}_{i}$. A sample data set is shown in Figure 28 where the different colors represent each of five states. The total model $R^{2}$ (Eq. (6.31)) is calculated using the set of points made up by all of the colors, i.e. the entire set of normalized data as defined in Eq. (6.30).

$$
\begin{gathered}
\left\|y_{i}\left(t_{k}\right)\right\|=\frac{y_{i}\left(t_{k}\right)}{\max \left(\left|\vec{y}_{i}\right|\right)} \quad \begin{array}{l}
i=1,2, \ldots N x \\
k=1,2, \ldots N
\end{array} \\
\left\|\hat{y}_{i}\left(t_{k}\right)\right\|=\frac{\hat{y}_{i}\left(t_{k}\right)}{\max \left(\left|\vec{y}_{i}\right|\right)} \quad \begin{array}{l}
i=1,2, \ldots N x \\
k=1,2, \ldots N
\end{array} \\
\vec{y}_{T}=\left[\begin{array}{llll}
\vec{y}_{1} & \vec{y}_{2} & \cdots & \vec{y}_{i}
\end{array}\right]^{T} \quad i=N x \\
\hat{\bar{y}}_{T}=\left[\begin{array}{llll}
\hat{\bar{y}}_{1} & \hat{\bar{y}}_{2} & \ldots & \hat{\bar{y}}_{i}
\end{array}\right]^{T} \quad
\end{gathered}
$$




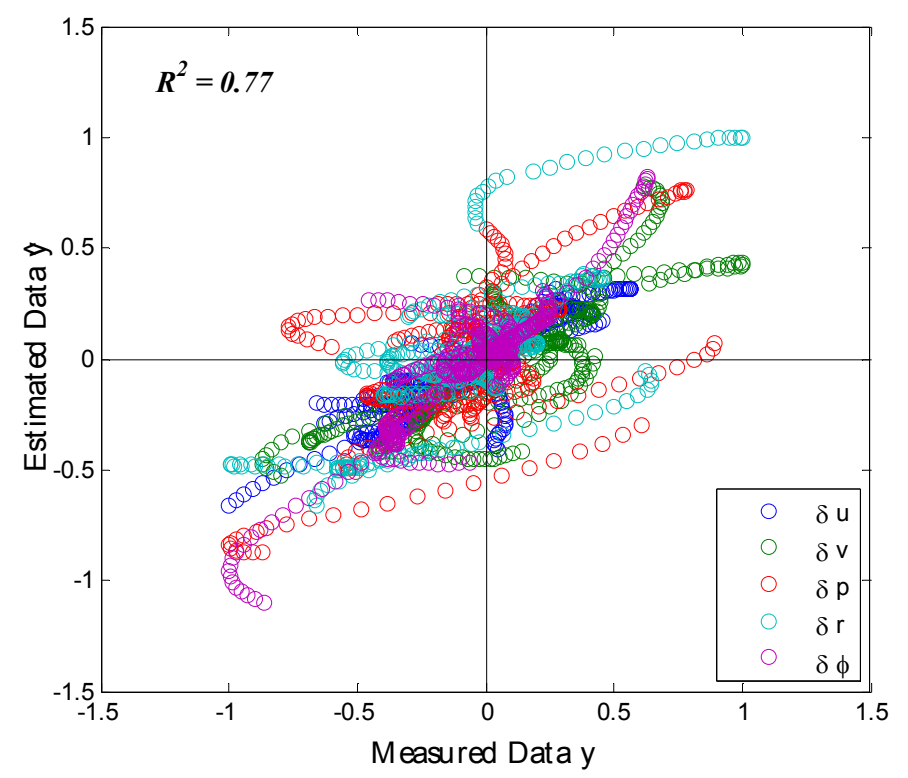

Figure 28: Example of what normalized data looks like for calculation of $\boldsymbol{R}^{2}$

$$
R_{T}^{2}=\left[\frac{\sigma_{y_{T} \hat{y}_{T}}}{\sigma_{y_{T}} \sigma_{\hat{y}_{T}}}\right]^{2}
$$

The disadvantage of $R^{2}$ is that perfect correlation $\left(R^{2}=1\right)$ does not necessarily indicate a perfect model fit, but only the existence of the exact linear relation of Eq. (6.27). In the case of a perfect model fit $\alpha=0$ and $\beta=1$, thus $\hat{y}_{i}\left(t_{k}\right)=y_{i}\left(t_{k}\right)$. Another measure of model fit which has frequently been used in economic forecasting is the Theil inequality coefficient(Theil, 1958). As it is defined in Eq. (6.32), it represents the ratio of the root mean square fit error and the root mean square values of the measured and estimated signals.

$$
U_{i}=\frac{\sqrt{1 / N \sum_{k=1}^{N}\left(y_{i}\left(t_{k}\right)-\hat{y}_{i}\left(t_{k}\right)\right)^{2}}}{\sqrt{1 / N \sum_{k=1}^{N}\left(y_{i}\left(t_{k}\right)\right)^{2}}+\sqrt{1 / N \sum_{k=1}^{N}\left(\hat{y}_{i}\left(t_{k}\right)\right)^{2}}} \quad i=1,2, . . N x
$$

The Theil inequality coefficient is bounded between 0 and 1 . For the case of a perfect model fit, $U_{i}=0$ for all $i$ (all states). The case where $U_{i}=1$ is referred to as the maximum of inequality, where there is negative proportionality between the points $\left(\vec{y}_{i}, \hat{\bar{y}}_{i}\right)$. It is accepted that models which exhibit a Theil inequality coefficient of 0.3 or less indicate a good model fit (Jategaonkar, 2006; Jategaonkar, Fischenberg, \& von Gruenhagen, 2004; Theil, 1958). The inequality 
coefficient itself is sensitive to additive variations, meaning if a constant is added to the same series of data, the Theil inequality coefficient will decrease falsely indicating a better model fit. This problem is avoided when all models measure data from the same reference point, as is the case of linear model data wherein all points in a time series are measured as perturbations around zero.

Theil offers a weighted value for the inequality coefficient that results in a single number for an entire model as formulated in Eq.(6.33), which is more suited to analyzing the entire model performance than the $R^{2}$ discussed earlier because it weights each signal according to its mean square error.

$$
U_{T}=\frac{\sum_{i=1}^{N x} U_{i}\left(\sqrt{\sum_{k=1}^{N}\left(y_{i}\left(t_{k}\right)\right)^{2}}+\sqrt{\sum_{k=1}^{N}\left(\hat{y}_{i}\left(t_{k}\right)\right)^{2}}\right)}{\sum_{m=1}^{N x}\left(\sqrt{\sum_{k=1}^{N}\left(y_{i}\left(t_{k}\right)\right)^{2}}+\sqrt{\sum_{k=1}^{N}\left(\hat{y}_{i}\left(t_{k}\right)\right)^{2}}\right)}
$$

One advantage to using Theil's approach to model evaluation is that the inequality coefficient may be broken down into partial coefficients due to "unequal central tendency, to unequal variation, and to imperfect covariation as shown in (6.34).

$$
U_{M}^{2}+U_{S}^{2}+U_{C}^{2}=U^{2}
$$

Where $U_{M}, U_{S}$, and $U_{C}$ are decompositions of the second moment of prediction errors and are formulated in Eq. (6.35)

$$
U_{M_{i}}=\frac{\bar{y}_{i}-\hat{\bar{y}}_{i}}{D} \quad U_{S_{i}}=\frac{\sigma_{y_{i}}-\sigma_{\hat{y}_{i}}}{D} \quad U_{C_{i}}=\frac{\sqrt{2\left(1-r_{i}\right) \sigma_{y_{i}} \sigma_{\hat{y}_{i}}}}{D}
$$

Where $D$ is the denominator of $U_{i}$ and $r_{\mathrm{i}}$ is the linear correlation coefficient in Eq. (6.25). If these are written as follows in Eq. (6.36), they become proportions which add up to one, as shown in Eq. (6.37).

$$
\begin{gathered}
U_{i}^{M}=\frac{U_{M_{i}}^{2}}{U_{i}^{2}} \quad U_{i}^{S}=\frac{U_{S_{i}}^{2}}{U_{i}^{2}} \quad U_{i}^{C}=\frac{U_{C_{i}}^{2}}{U_{i}^{2}} \\
U_{i}^{M}+U_{i}^{S}+U_{i}^{C}=1
\end{gathered}
$$


These proportions are very useful because they will provide information as to possible sources of error in the model estimation. The full formula for calculating each of these is given in Eqs. (6.38) - (6.39).

$$
\begin{aligned}
& U_{i}^{M}=\frac{N\left(\bar{y}_{i}-\hat{\bar{y}}_{i}\right)^{2}}{\sum_{k=1}^{N}\left[y_{i}\left(t_{k}\right)-\hat{y}_{i}\left(t_{k}\right)\right]^{2}} \\
& U_{i}^{S}=\frac{N\left(\sigma_{y_{i}}-\sigma_{\hat{y}_{i}}\right)}{\sum_{k=1}^{N}\left[y_{i}\left(t_{k}\right)-\hat{y}_{i}\left(t_{k}\right)\right]^{2}} \\
& U_{i}^{C}=\frac{2\left(1-r_{i}\right) \sigma_{\hat{y}_{i}} \sigma_{y_{i}}}{\sum_{k=1}^{N}\left[y_{i}\left(t_{k}\right)-\hat{y}_{i}\left(t_{k}\right)\right]^{2}}
\end{aligned}
$$

The first, $U^{M}$, is termed the bias proportion and represents a measure of the systematic error in the identified model and should be zero if the estimated model is on the average correct. The second, $U^{S}$, is termed the variance proportion and represents the model's ability to duplicate the variability in the true system. The variance proportion should also be zero in the ideal case which would indicate there is no variation in the estimated model. The last, $U^{C}$, is the covariance proportion and represents a measure of nonsystematic error. It is not expected that any kind of mathematical model, no matter how complex, will be able to perfectly predict the behavior of the true system, and we hope that this is the only source of error in our model. Thus the ideal value for the covariance proportion is one (Theil, 1958).

The ideal case defined in the previous paragraph of $U^{M}=U^{S}=0$ and $U^{C}=1$ is not achievable, but from the ideal case it is clear that large values of the bias and variance proportion are unsatisfactory. If either of these is proportions climbs higher that 0.1 there is an indication that there could be a problem not only with the estimated model, but also with the measured data as well (Jategaonkar, 2006). For each estimated model, the three proportions will be calculated for each state both as a means of verifying a good model fit as well as a way to investigate potential problems in the measured data. 


\section{Non-Linear Model Estimation}

As mentioned in Chapter 3, full non-linear model identification entails analyzing data through the full operating range of the boat. In order to do this accurately, it is necessary to have fairly precise knowledge of parameters such as vehicle mass, moments of inertia, and center of gravity. The test yacht is a boat designed in an era before computer aided design and after contacting the designer of the boat, not all of these parameters could be obtained. Tests were performed on Avanti to try and determine these numbers, but the results were still not considered very accurate estimates. For small aircraft, colleagues at the University of Kansas have produced good results for obtaining such parameters by slinging aircraft and performing dynamic swing tests on multiple axes. This was not practical within current facilities for a boat as large as Avanti. A detailed 3D solid CAD model is generated by the author for Avanti, but in the absence of experimental validation even this model was believed to be unreliable for the purpose of nonlinear model identification. Given this challenge and the inherent challenge of non-linear estimation, an attempt was not made to fully characterize a non-linear model for the test yacht with the full-scale data. It was determined that that task is better suited to a future study with a purpose-built boat. Thus, the only non-linear estimation performed for this dissertation uses the non-linear simulation data. The details of this analysis are given herein.

\subsection{MLE Estimation of Non-Linear Hydrodynamic Derivatives}

The data generated by the simulation presented in Section 3.5 has been used to test the MLE method to obtain hydrodynamic derivatives. Simulation data is best for testing the algorithm because it is generated by a 'perfect' description of the model, is completely uncorrupted by noise, and the parameters are known and may be compared to estimates. None of the above is true for the real data, but the simulation is a useful tool for designing and testing maneuvers perform with the full-scale system. MATLAB's ode113 integrator is employed to integrate the state equations during the estimation, and the optimization procedure is done following the Levenberg-Marquardt method. The perturbation step size used to vary parameters is 1e-3, and the convergence limit is set to a total relative change in the cost function of 1e-4.

The state equations that are integrated during estimation represent the 4DOF model as defined in Eqs. (3.45) - (3.48). The control vector, given in Eq. (7.1), is the only place in the 
estimation procedure where external time histories are employed, so the control vector includes the rudder angle, apparent wind speed, and apparent wind angle. This non-linear model does not include the new aerodynamic model so the sail angle is not included as an input, and is rather treated as always trimmed for maximum thrust.

$$
\vec{u}\{k\}=\left[\begin{array}{lll}
\delta_{R}\{k\} & V_{A}\{k\} & \beta_{A}\{k\}
\end{array}\right]^{T}
$$

In this equation $k$ is the time index, as the system inputs are discrete time measurements. The output vector, or the vector of measurements, includes the accelerations and the states:

$$
\vec{y}=\left[\begin{array}{llllllllll}
\dot{U} & \dot{V} & \dot{P} & \dot{R} & U & V & P & R & \phi & \psi
\end{array}\right]^{T}
$$

For this test, the unknown parameter vector is the set of hydrodynamic derivatives:

$$
\vec{\beta}=\left[\begin{array}{llllllll}
X_{V V} & X_{\phi \phi} & Y_{V} & Y_{\phi} & K_{V} & K_{\phi} & N_{V} & N_{\phi}
\end{array}\right]^{T}
$$

The purpose of this test is to show that the rudder maneuvers that have been performed at full-scale will give the estimator enough excitation in the data to estimate the hydrodynamic derivatives. It is possible to estimate more parameters than this, but that significantly increases the time it takes for the algorithm to reach convergence on a unique solution. So for this

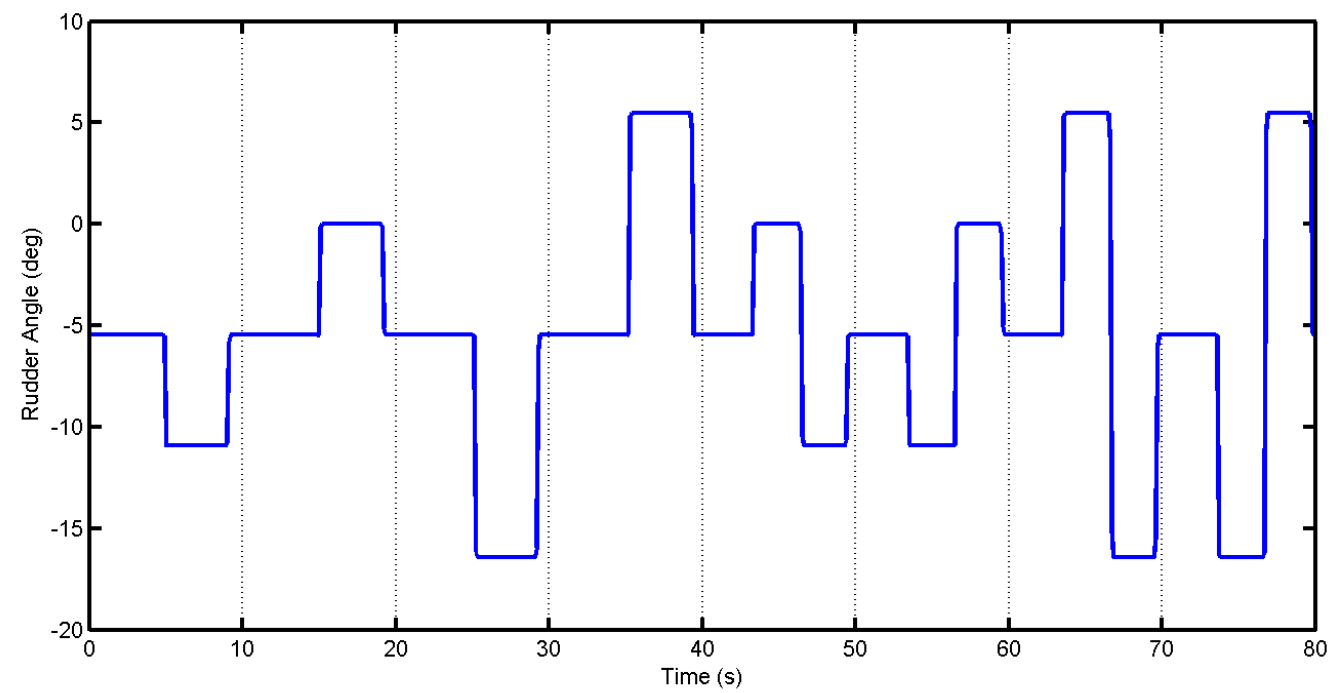

Figure 29: Rudder input sequence used in non-linear simulator to generate estimation data. 
estimation, it is assumed all the aerodynamic and other system parameters are known.

The total data set used for this estimation consisted of measurements at 808 points in time. The input sequence consisted of 8 maneuvers tested in simulation, 4 rudder singlets and 4 rudder doublets. These are shown in Figure 29. The data was generated by running the simulation 8 times, each with a different rudder maneuver. The data generated is then is lumped into one timeseries, with initial conditions for state equation integration re-set at the beginning of each maneuver for the estimation. It takes a standard PC running MATLAB about 40 minutes to converge on a solution after 39 iterations. The initial parameter guesses, actual values, and results of the estimation procedure including standard deviations of each estimate are shown in Table 6.

For the sailing yacht, the initial parameter guesses must be very close or the same order of magnitude as the actual values, otherwise the integrator tends to diverge quickly, making the estimation impossible. The parameters reported in Table 6 do not match perfectly, but the resultant model is to be judged by its ability to predict future motion, which is shown in Figure 30. The vertical dashed lines distinguish between the different maneuvers, which is why there are discontinuities at these points. As may be seen in Figure 30, the estimated model is tracking perfectly in $U, P$, and $\phi$; moderately well in $V$ and $R$, and not very well at all in $\psi$. All of the data generated by the simulation for this analysis was done starts in the same trim condition, same boat speed and true wind. It will likely be possible to improve the results of the estimation further by adding more data from different trim conditions. The initial result of this attempt to estimate a simple set of hydrodynamic parameters for the sailing yacht shows that it will be difficult to obtain the exact values of the hydrodynamic derivatives, because, as shown in Table 6 some of the derivatives are different order of magnitude than the actual value, and in some cases they have actually changed signs. 
Table 6: Summary of MLE Estimation of Hydrodynamic Derivatives

\begin{tabular}{|c|r|c|c|c|}
\hline \multirow{2}{*}{ Param } & $\begin{array}{c}\text { Initial } \\
\text { Guess }\end{array}$ & $\begin{array}{c}\text { Actual } \\
\text { Value }\end{array}$ & $\begin{array}{c}\text { Estimated } \\
\text { Value }\end{array}$ & Std. Dev. \\
\hline \hline$X_{V V}^{\prime}$ & $5 \mathrm{e}-1$ & $3.38 \mathrm{e}-1$ & $2.95 \mathrm{e}-1$ & $1.97 \mathrm{e}-4$ \\
\hline$X_{\phi \phi}^{\prime}$ & $5 \mathrm{e}-3$ & $1.40 \mathrm{e}-3$ & $1.56 \mathrm{e}-2$ & $5.94 \mathrm{e}-5$ \\
\hline$Y_{V}^{\prime}$ & $-5 \mathrm{e}-1$ & $-5.35 \mathrm{e}-1$ & $-5.72 \mathrm{e}-1$ & $2.26 \mathrm{e}-4$ \\
\hline$Y_{\phi}^{\prime}$ & $-5 \mathrm{e}-3$ & $-5.89 \mathrm{e}-3$ & $1.81 \mathrm{e}-3$ & $1.17 \mathrm{e}-6$ \\
\hline$K_{V}^{\prime}$ & $5 \mathrm{e}-1$ & $2.80 \mathrm{e}-1$ & $3.07 \mathrm{e}-2$ & $1.55 \mathrm{e}-4$ \\
\hline$K_{\phi}^{\prime}$ & $5 \mathrm{e}-3$ & $3.36 \mathrm{e}-3$ & $-3.07 \mathrm{e}-2$ & $1.12 \mathrm{e}-5$ \\
\hline$N_{V}^{\prime}$ & $-5 \mathrm{e}-2$ & $-3.23 \mathrm{e}-2$ & $-4.98 \mathrm{e}-2$ & $2.84 \mathrm{e}-5$ \\
\hline$N_{\phi}^{\prime}$ & $-5 \mathrm{e}-2$ & $-1.52 \mathrm{e}-2$ & $-4.76 \mathrm{e}-2$ & $2.18 \mathrm{e}-5$ \\
\hline & & & & \\
\hline
\end{tabular}

This preliminary application of system identification to estimate non-linear hydrodynamic derivatives of a sailing yacht has yielded satisfactory results in terms of the scope of this project. The pairing of the sailing yacht non-linear model structure and MLE formulation is unique, and may be used in the future with any data containing the necessary measurements. The MLE algorithm does an excellent job of matching the model output, which is important for control system design. Future work with the simulation data could explore using data from different trim conditions and a set of simulation data that more closely resembles the standard ziz-zag maneuver used for ships. If the results obtained through the estimation procedure using the simulation data can be improved, this method may be applied to comparative full-scale data. 

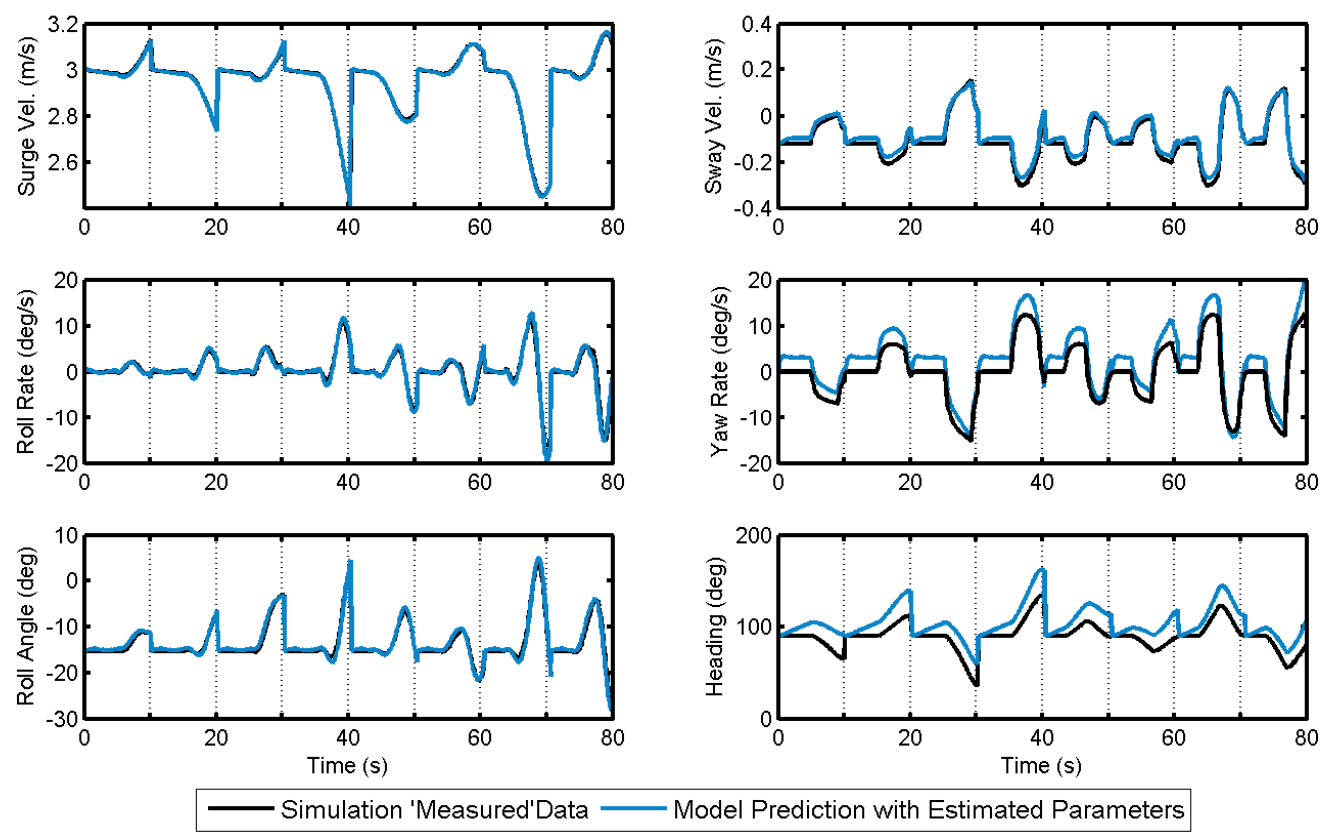

Figure 30: Time history of simulated and estimated non-linear hydrodynamic model 


\section{Linear Model Estimation}

This Chapter begins with a detailed definition of the linear model structure that has been estimated from both simulation and full scale data. Section 8.1.1 demonstrates the applicability of the OSLS to estimation of the linear sailing yacht dynamic model through use of simulation data. The OSLS algorithm does not handle signal noise very well, so the data must be specially prepared for OSLS estimation. The details of this process are given in Section 8.2. The estimation process and resulting models estimated from the full-scale data are detailed in Section 8.3. All the models estimated in this Chapter have the following structure:

$$
\begin{aligned}
\dot{\bar{x}}(t) & =A \vec{x}(t)+B \vec{u}(t) \quad \vec{x}\left(t_{0}\right)=\vec{x}_{0} \\
\vec{y}(t) & =\vec{x}(t)
\end{aligned}
$$

where:

$$
\dot{\vec{x}}=\left[\begin{array}{c}
\Delta \dot{u} \\
\Delta \dot{v} \\
\Delta \dot{p} \\
\Delta \dot{r} \\
\Delta \dot{\phi}
\end{array}\right] \quad \vec{x}=\left[\begin{array}{c}
\Delta u \\
\Delta v \\
\Delta p \\
\Delta r \\
\Delta \phi
\end{array}\right] \quad \vec{u}=\left[\begin{array}{c}
\Delta \delta_{r} \\
\Delta \delta_{b}
\end{array}\right] \quad \vec{x}_{0}=\left[\begin{array}{l}
u \\
v \\
p \\
r \\
\phi
\end{array}\right] \quad \vec{u}_{0}=\left[\begin{array}{l}
\delta_{r} \\
\delta_{b}
\end{array}\right]
$$

and where the perturbations, as in Chapter 4, are denoted by the small letters, and the total measured variables are denoted by capital letters:

$$
\begin{aligned}
& U=u+\Delta u \\
& V=v+\Delta v \\
& P=p+\Delta p \\
& R=r+\Delta r \\
& \Phi=\phi+\Delta \phi \\
& \delta_{R}=\delta_{r}+\Delta \delta_{r} \\
& \delta_{B}=\delta_{b}+\Delta \delta_{b}
\end{aligned}
$$




\subsubsection{Models Estimated From Simulation Data}

First this method was applied to a set of data generated by the linear model in Eq. (8.5) with eigenvalues given by Eq. (8.6). This linear model was calculated based on the numerical method discussed in Section 4.3:

$$
\begin{aligned}
& \vec{x}_{0}=\left[\begin{array}{lllll}
3.0 \frac{\mathrm{m}}{\mathrm{s}} & -0.12 \frac{\mathrm{m}}{\mathrm{s}} & 0.00 \frac{\mathrm{rad}}{\mathrm{s}} & 0.00 \frac{\mathrm{rad}}{\mathrm{s}} & -0.27 \mathrm{rad}
\end{array}\right]^{T} \\
& \vec{u}_{0}=\left[\begin{array}{ll}
-0.01 \mathrm{rad} & 0.17 \mathrm{rad}
\end{array}\right]^{T} \\
& {\left[\begin{array}{c}
\dot{u} \\
\dot{v} \\
\dot{p} \\
\dot{r} \\
\dot{\phi}
\end{array}\right]=\left[\begin{array}{ccccc}
-.0191 & -0.306 & 0 & -0.285 & 0.055 \\
0.153 & -0.648 & -0.076 & 0.874 & -0.049 \\
-0.050 & 0.387 & 0 & -0.697 & -1.972 \\
-0.032 & -1.472 & 0 & -3.511 & -0.445 \\
0 & 0 & 1 & 0 & 0
\end{array}\right]\left[\begin{array}{c}
\Delta u \\
\Delta v \\
\Delta p \\
\Delta r \\
\Delta \phi
\end{array}\right]+\left[\begin{array}{cc}
0.087 & -0.989 \\
-1.839 & 0.653 \\
0.473 & 0.992 \\
2.386 & -0.021 \\
0 & 0
\end{array}\right]\left[\begin{array}{l}
\Delta \delta_{r} \\
\Delta \delta_{b}
\end{array}\right]} \\
& \lambda=\left\{\begin{array}{llll}
-2.91 & -1.23 & -0.22 & 0.003 \pm 1.40 i
\end{array}\right\}
\end{aligned}
$$

The estimated model exactly matches the linear simulation model which was used to generate the data for identification. This is expected and demonstrates the potential accuracy of the OSLS formulation when given perfect data from a known model.

Next, data generated by the non-linear simulation was used to estimate a linear model, more accurately representing the reality that the linear model is an oversimplification of the real physical non-linear system. The estimated linear model is given in Eq. (8.7), its initial conditions are given by Eq. (8.4), and the eigenvalues given by Eq. (8.8).

$$
\begin{gathered}
{\left[\begin{array}{c}
\dot{u} \\
\dot{v} \\
\dot{p} \\
\dot{r} \\
\dot{\phi}
\end{array}\right]=\left[\begin{array}{ccccc}
-0.036 & -2.348 & 0.108 & -2.97 & -0.129 \\
0.034 & 1.786 & -0.094 & 3.769 & 0.084 \\
-0.169 & 3.522 & -0.141 & 3.467 & -1.750 \\
0.024 & -1.227 & -0.037 & -3.241 & -0.176 \\
0 & 0 & 1 & 0 & 0
\end{array}\right]\left[\begin{array}{c}
\Delta u \\
\Delta v \\
\Delta p \\
\Delta r \\
\Delta \phi
\end{array}\right]+\left[\begin{array}{cc}
0.450 & -0.168 \\
-2.366 & -0.044 \\
-0.388 & -0.350 \\
2.478 & 0.323 \\
0 & 0
\end{array}\right]\left[\begin{array}{c}
\Delta \delta_{R} \\
\Delta \delta_{B}
\end{array}\right]} \\
\lambda=\left\{\begin{array}{lll}
-2.01 & 0.13 \pm 0.30 i & 0.06 \pm 1.45 i
\end{array}\right\}
\end{gathered}
$$

The time history response of the measured and estimated model is shown in Figure 31. The resulting fit of the estimated model is $R^{2}$ of 0.96 and $U^{T}$ of 0.08 . These numbers represent an excellent model fit, and it is not expected in this case that the estimated model should fit the data perfectly because the estimated linear model structure is an oversimplification of the known non- 

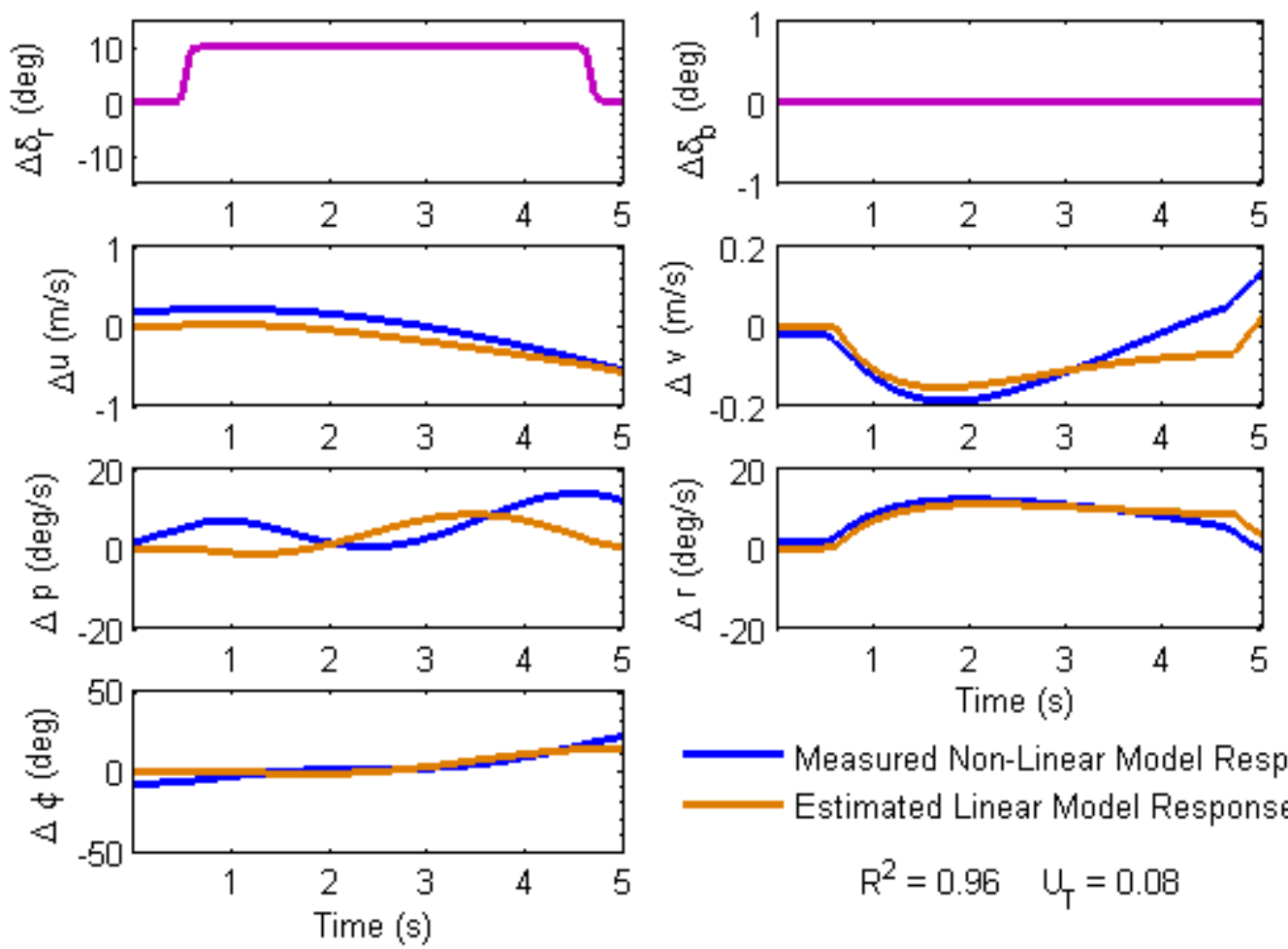

Measured Non-Linear Model Response

Estimated Linear Model Response

$\mathrm{R}^{2}=0.96 \quad \mathrm{U}_{\mathrm{T}}=0.08$

Figure 31: Comparison of the response of the estimated linear model to the 'Measured' nonlinear simulation data.

linear model structure. The implications of the values of $R^{2}$ of 0.96 and $U^{T}$ of 0.08 are further discussed in Section 8.3.

The first linear model, Eqs. (8.5) and (8.6), has three stable first order modes and an unstable second order mode. The second linear model, Eqs. (8.7) and (8.8), has only one stable first order mode and two unstable second order modes.

\subsection{Preparation of Full-scale Data for Linear System Identification}

The data summarized in Section 5.3 is contained in several very long files of continuous sailing. In order to use the data for system identification, each of these files is manually examined for maneuvers. The process is illustrated in Figure 32 where the blue lines represent about 7 minutes of sailing data during which three sail maneuvers and four rudder doublets were performed. The red (bold) highlights one of the sections chosen for a sail maneuver time 

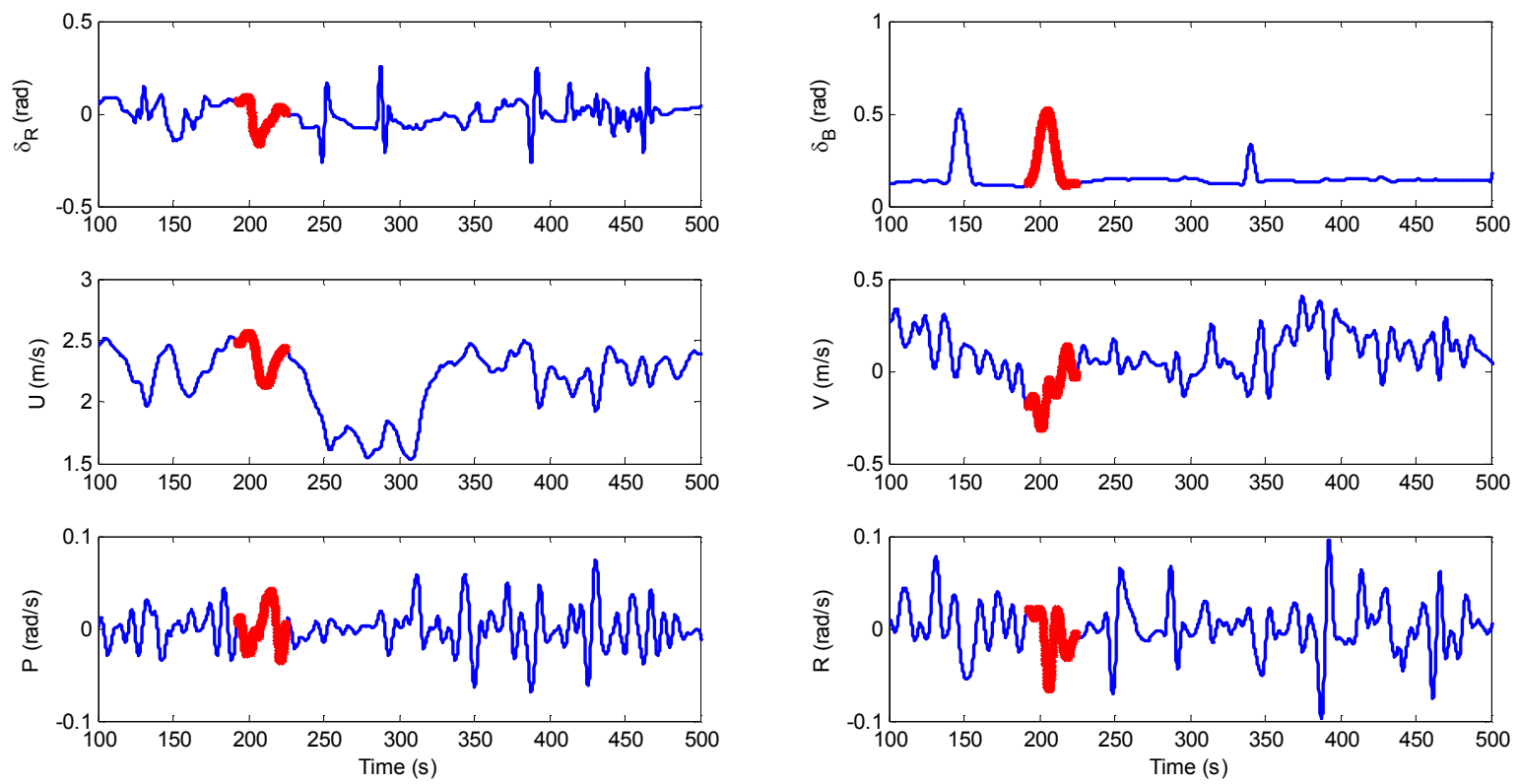

Figure 32: Sample of maneuver segment in full data file.

segments. Identifying the maneuver segments is extremely time intensive, but once the data has been put into this usable format estimation using OSLS and analysis is very quick.

Each individual maneuver segment is anywhere from $30-60$ seconds in length. The length of each maneuver segment varies according to the time it took for the vehicle to return to the initial steady state sailing condition. Sometimes insufficient time was allotted on the water while sailing for the boat to fully return to the initial condition before another maneuver was initiated, so maneuvers that are actually used for estimation are carefully chosen to avoid unwanted helmsman-induced control responses. This difficulty was particularly true in the case of the surge velocity response, as it is the slowest response of the five states. Typically the boat slows down during a commanded maneuver and takes a relatively long time to come back up to speed, something that was difficult to sense while out sailing on the water. Future tests should feed the surge velocity back to the helmsman to provide a quantitative assessment that the boat is allowed sufficient time to recover its forward velocity, such that subsequent maneuvers are not prematurely initiated.

To prepare the data for linear estimation, the variables must be transformed into the state vector described by Eq. (6.4) using initial conditions. For this work, it has been assumed that the initial conditions represent the sailing trim state of the sailing yacht. Each maneuvering segment 
has time before the maneuver begins during which the boat was sailing in a balanced, steady state condition. This time varies and has been manually recorded for each individual maneuver based upon how long the state variable remained relatively constant before the actual control surface deflection begins. The state variables are averaged over this segment of time to determine the initial conditions defined for that maneuver. Lastly, each maneuvering segment contains the index time at which the control surface deflection actually begins, as this point is where the open loop simulation starts when the model fit is evaluated.

The OSLS estimation procedure is extremely sensitive to signal noise, thus the raw data maneuvering segments have been altered for use with this estimation technique. The goal of preprocessing the raw data is to remove any sharp discontinuities in the signals as shown in the $\delta_{\mathrm{B}}$ signal of Figure 33a, and to remove as much of the signal oscillation as much as possible without losing information about the actual vehicle response. With these goals in mind, the raw data is smoothed using a robust locally weighted polynomial regression technique (LOESS)(Cleveland, 1979). The process has a parameter $f$ which when set less than 0.01 , essentially returns the original signal, thus there is much control over the degree of smoothing. The smoothing function works by initially fitting a second order polynomial using weighted least squares to the data, then assigning weights to each point, with higher weights for points closer to the polynomial and lower weights for points farther from the polynomial. A new polynomial fit is calculated, new weights are calculated, and the process is repeated several times to achieve the best fit. For smoothing the raw sailing data, the $f$ value is chosen separately for each variable, and ranges from 0.09 to 0.25 based on visual inspection of each signal such as the examples of smoothed data signals shown in Figure 33. As may be seen especially in the rudder doublet, sometimes the smoothed data does not always fully capture the peak. It is likely in some cases key response information is lost through this smoothing process. There are two ways to address this in the future: by using a either frequency-based filtering strategy with the data or an estimation algorithm like MLE which can handle the noisy signals. 
(a)
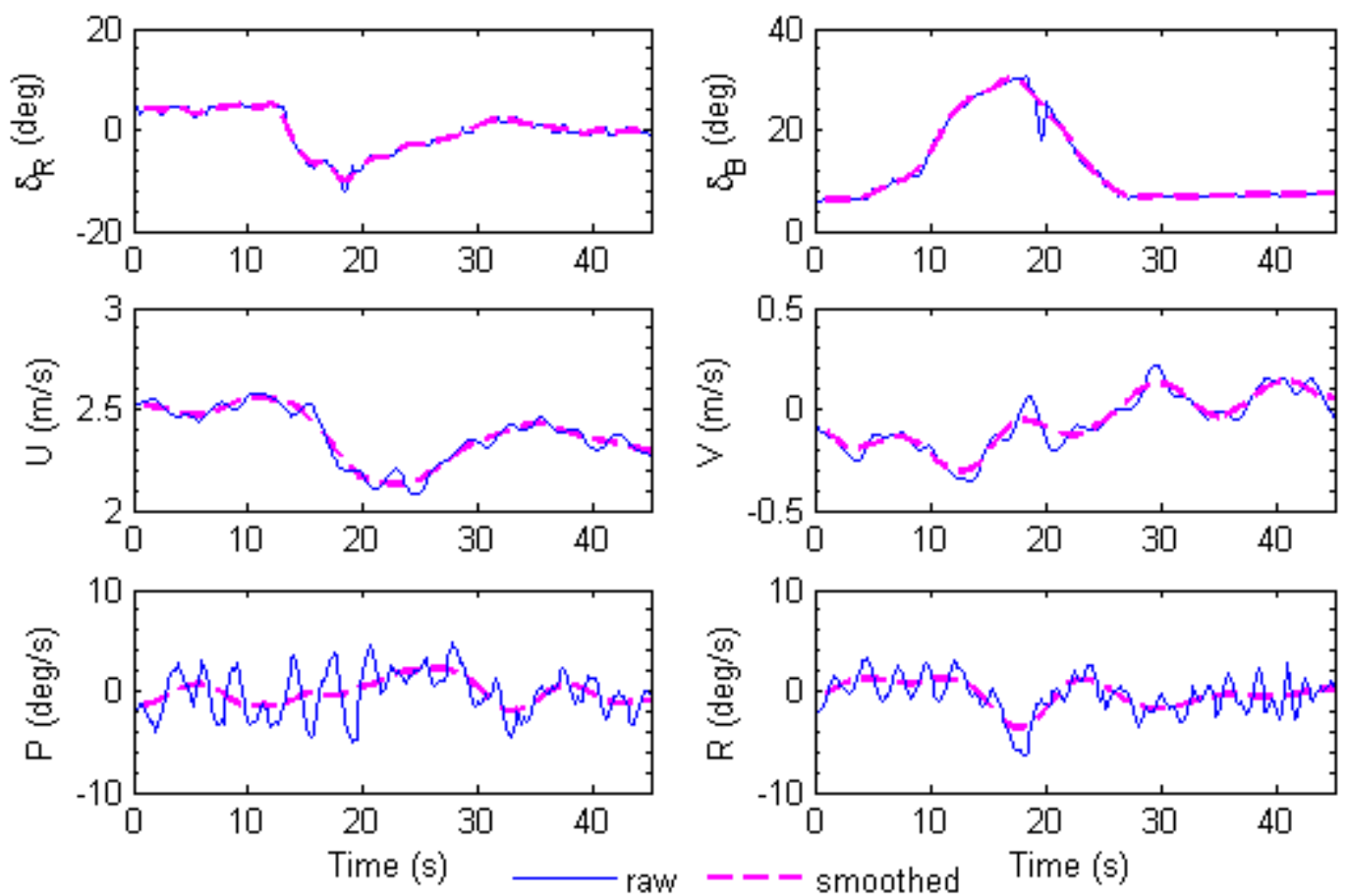

\section{(b)}
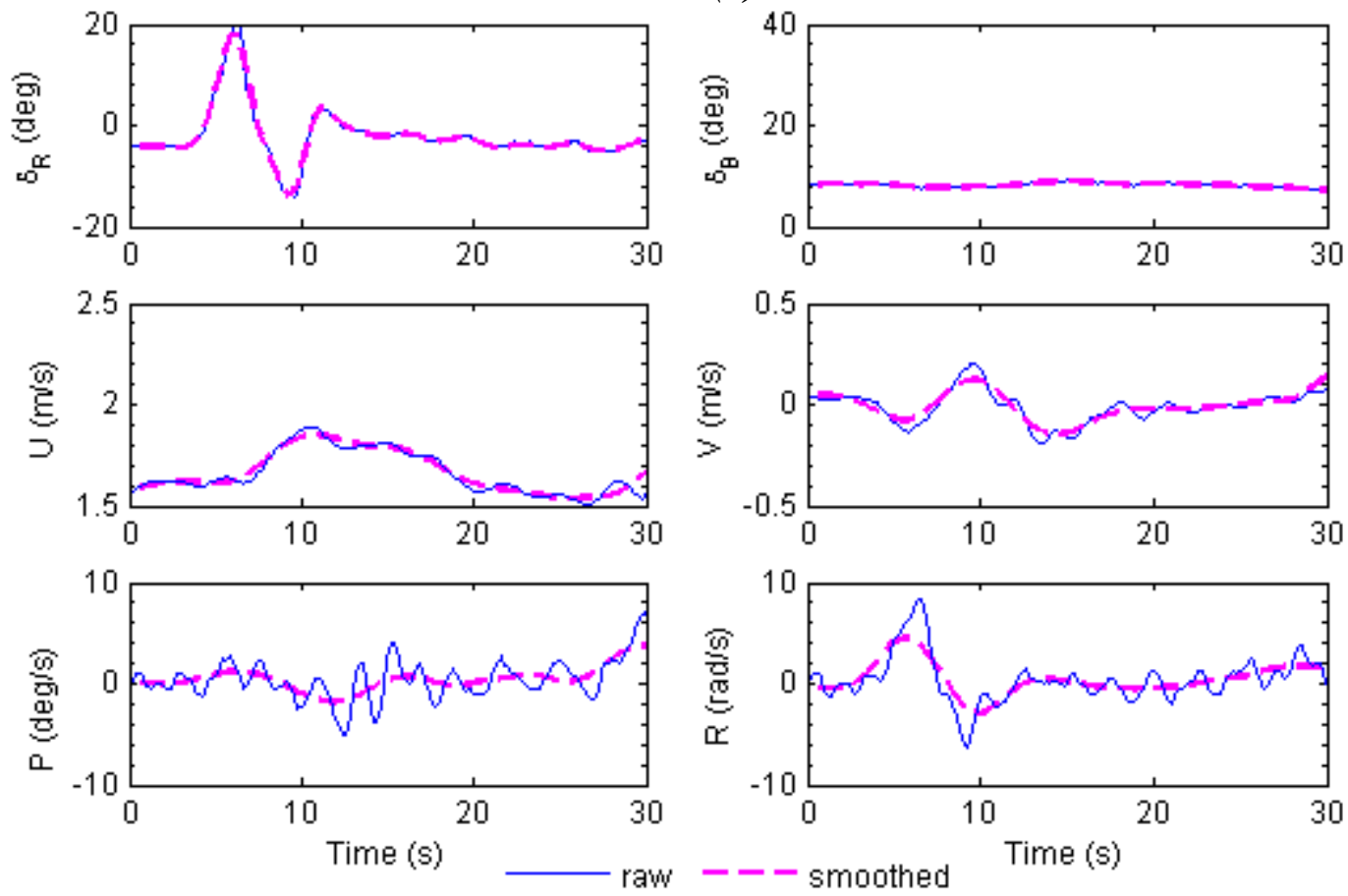

Figure 33: Sample set of smoothed maneuver data sets for (a) a rudder and sail input and (b) for a rudder doublet. 


\subsection{Estimation of Linear Models from Full-Scale Data}

The full-scale data is separated by sail configuration into the following three categories, again relating to the sail configuration of the boat as mentioned in Section 5.3: Genoa, Jib, and Reef maneuvers. Thus there are three sets of estimated models for the full-scale boat representing the boat in the three different sail configurations. The models in each of these configurations represent a range of initial conditions, the implications of which are discussed in Sections 8.4, 8.5, and 8.6. The rest of this sub-section is devoted to describing the analysis process used to estimate, validate, and analyze the modes of the linear models obtained from the full-scale data.

In the interest of attaining a large number of sample models, the data was grouped into all possible combinations of three maneuvers, with each grouping containing one rudder singlet, one rudder doublet, and one sail singlet. This is only done for the Genoa and Jib configurations, as there was not enough data in the Reef configuration for this kind of analysis. A sample combination of ID data for the Genoa configuration is shown in Figure 34, and a sample combination of ID data for the Jib configuration is shown in Figure 35. Once the sets of ID data
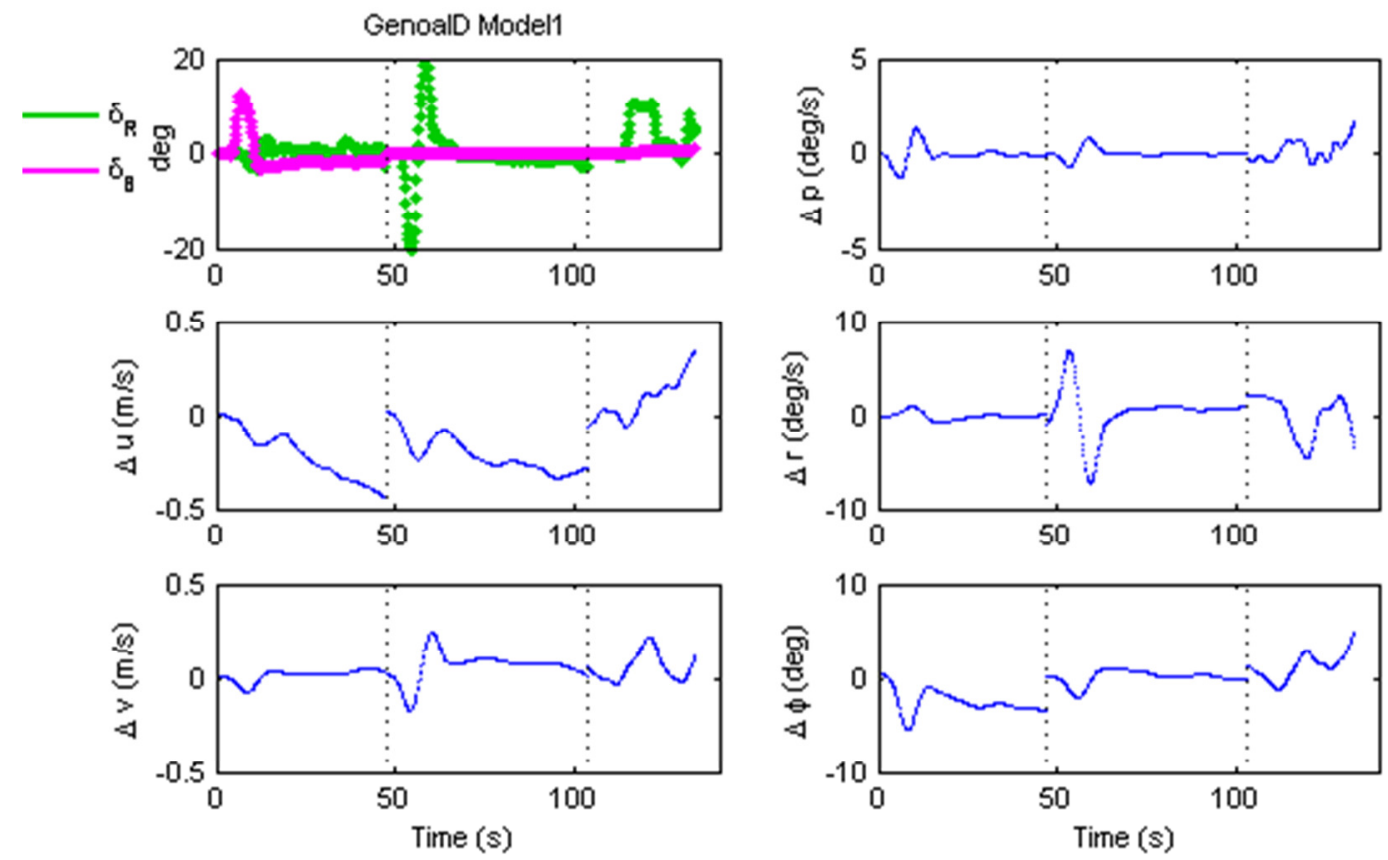

Figure 34: Sample set of ID data for the Genoa configuration with one boom singlet, one rudder doublet, and one rudder singlet. 

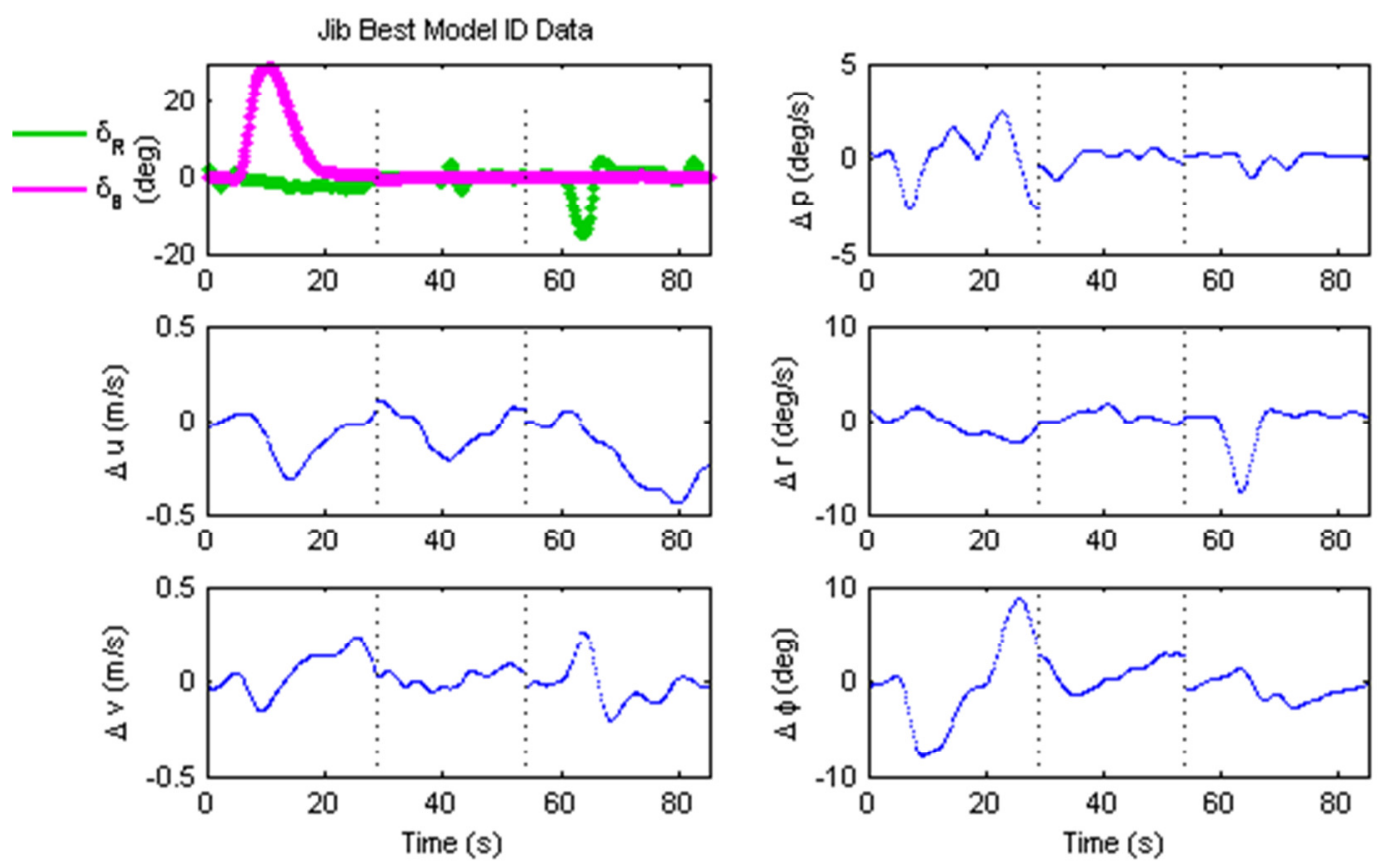

Figure 35: Sample set of ID data for the Jib configuration with one sail singlet, one rudder doublet, and one rudder singlet.

are composed, the OSLS algorithm outlined in the beginning of this Chapter is used to estimate the linear models for each set of composite maneuvers. Each set of ID data is matched with an independent set of ID data, which then becomes the TEST data associated with the ID data.

Each estimated model is tested with both the ID data, and the independent set of TEST data in the open loop simulation. Valid models are those defined by having an $R^{2}$ greater than 0.75 and $U_{T}$ less than 0.3 for both the ID and TEST data open loop simulations. The $U_{T}<0.3$ criteria is a well-established rule of thumb for acceptable model fits (Theil, 1958). The $R^{2}>0.75$ criteria is chosen for several reasons. This is the first attempt at estimation of linear sailing yacht dynamic models, and a very simple estimation algorithm which neglects uncertainty in the model and noise in the measurements has been used to estimate models. Typical fit criteria for aircraft models would be at a minimum $R^{2}>0.9$ (Jategaonkar, 2006), which in our case would be considered a fabulous fit given the limitations of the estimation algorithm. It is not expected to estimate models at the same level of fit as aircraft because, unlike the situation with aircraft model identification, the model structure for the sailing yacht has some inherent uncertainty, the maneuvers were not optimally designed to excite system dynamics because the dynamics are not known a priori, and the data has been smoothed, likely at some small expense of dynamic 
response information. In addition to allowing for the errors introduced by the modeling and the measured maneuvers, the $R^{2}>0.75$ is considered acceptable for the slow time constants associated with the boat, which are all on the order of whole seconds, whereas it is typical for aircraft to exhibit modes with real roots with real magnitudes greater than one, corresponding to time constants on the order of fractions of a second. In terms of control design, the helmsman or computer control system aboard a sailing yacht has ample time to respond to changes and maintain control of the boat. Using the two criteria, Theil coefficient and $R^{2}$, together has been deemed appropriate for this proof-of-concept study.

The modes of each model are defined as the eigenvalues of the estimated system matrix (the A matrix). Valid models are then classified as one of two types, which have arbitrarily been called Type A and Type B models. Type A models exhibit one first order mode, and two second order modes, whereas Type B models exhibit three first order modes and one second order mode. Each mode is separated from the rest according to natural frequency. This breakdown of models is better described by Figure 36. For the Type A models, Mode 1A and Mode 2A are second

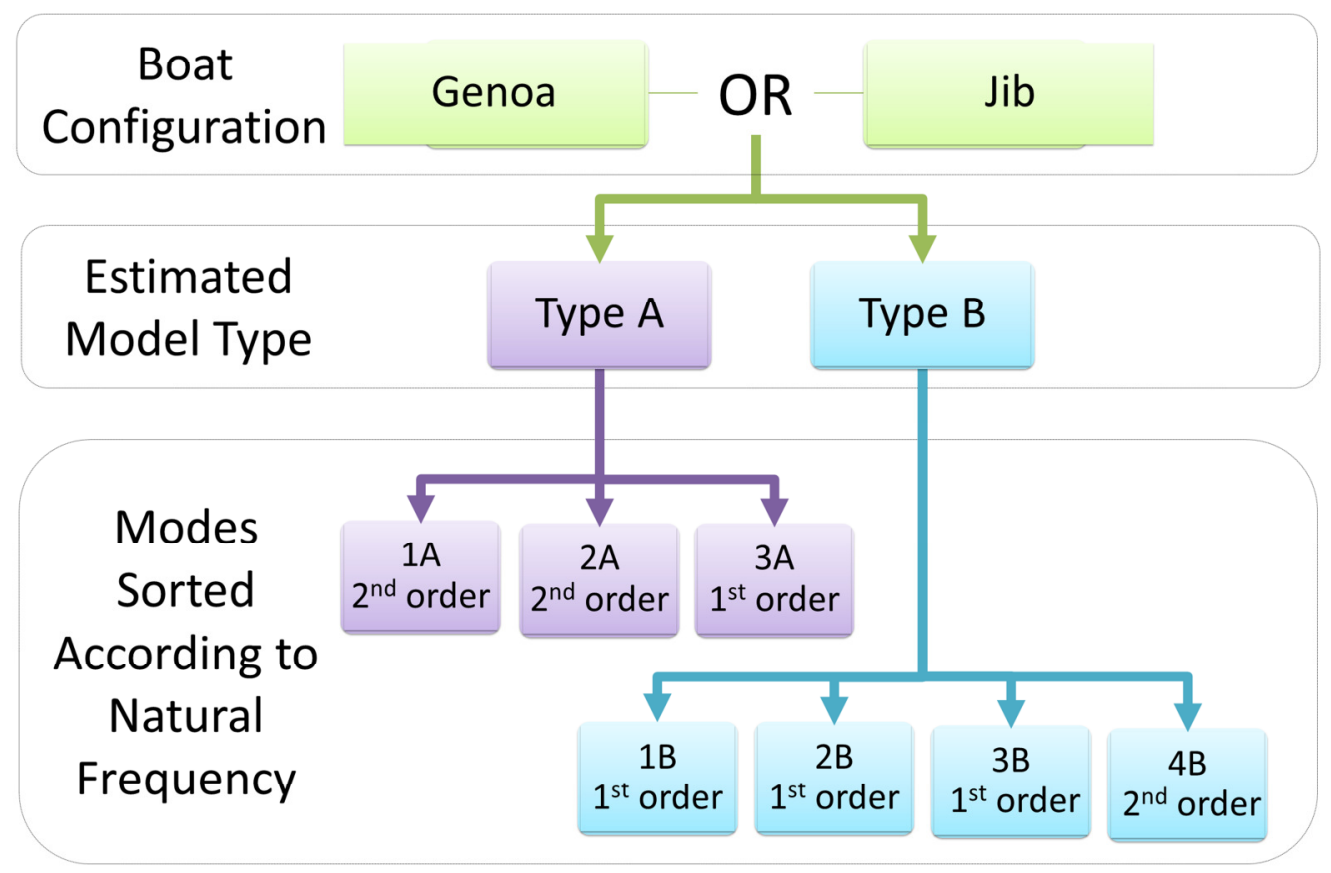

Figure 36: Breakdown of linear models by boat configuration, model type, modal order, and natural frequency. 
order, and Mode 3A is first order. For the Type B models, Mode 4B is second order, and Mode 1B, Mode 2B, and Mode 3B are first order. The naming convention was chosen before in depth analysis of each mode was begun, which is why the names follow a different order. To remind the reader of which modes are first and second order, when referred to in the text the numerical designation will be followed by a .S for second order modes, and a .F for first order, so second order Mode 1A will be designated Mode 1A.S.

The modes may be analyzed by looking at their corresponding eigenvectors, which are calculated from the estimated $A$ matrix. Second order modes will have complex valued eigenvectors as shown in Eq. (8.9).

$$
\mathbf{e}_{\lambda_{1}}=\left[\begin{array}{c}
\sigma_{u}+j \omega_{u} \\
\sigma_{v}+j \omega_{v} \\
\sigma_{p}+j \omega_{p} \\
\sigma_{r}+j \omega_{r} \\
\sigma_{\phi}+j \omega_{\phi}
\end{array}\right]
$$

MATLAB outputs each eigenvector, $\mathbf{e}_{\lambda_{n}}$ scaled such that the norm of each eigenvector is one. For this analysis only the magnitude of each complex value is examined, where the magnitude is calculated as in Eq. (8.10):

$$
M_{i}=\left|\sigma_{i}+j \omega_{i}\right|=\sqrt{\sigma_{i}^{2}+\omega_{i}^{2}} \quad i=1: N x
$$

The eigenvector of magnitudes is then defined in Eq. (8.11):

$$
\mathbf{v}_{\lambda_{1}}=\left[\begin{array}{c}
M_{u} \\
M_{v} \\
M_{p} \\
M_{r} \\
M_{\phi}
\end{array}\right]
$$

There are units associated with each of these eigenvectors because they come straight out of the estimated $A$ matrix. Therefore, each eigenvector is non-dimensionalized according to the SNAME Prime I system (Fossen, 2011) in Eq. (8.12): 


$$
\mathbf{v}_{\lambda_{1}}^{\prime}=\left[\begin{array}{c}
M_{u} / U \\
M_{v} / U \\
M_{p} U / L_{W L} \\
M_{r} U / L_{W L} \\
M_{\phi}
\end{array}\right]=\left[\begin{array}{c}
M_{u}^{\prime} \\
M_{v}^{\prime} \\
M_{p}^{\prime} \\
M_{r}^{\prime} \\
M_{\phi}^{\prime}
\end{array}\right]
$$

Finally, the non-dimensionalized eigenvector of magnitudes $\mathbf{v}_{\lambda_{1}}^{\prime}$ is scaled in Eq. (8.13) such that the sum of the elements is one, thus each entry will represent the percentage each state contributes to the particular mode.

$$
M_{u}^{\prime}+M_{v}^{\prime}+M_{p}^{\prime}+M_{r}^{\prime}+M_{\phi}^{\prime}=1
$$

The detailed modal analysis of eigenvectors is only done for Jib estimated models because there are the most valid estimated models in that category. For this reason, the Jib models are also presented first in Section 8.4, followed by the Genoa models in Section 8.5, and finally, the Reef model in Section 8.6.

\subsection{Estimated Jib Models}

A total of 1211 sets of ID and TEST data in the Jib configuration produced models which met the minimum $R^{2}$ and $U_{T}$ criteria in both the ID data fit and TEST data fit. Of these, 102 are unique valid models. This means that several of the sets of ID data matched more than one set of TEST data, indicating further that these models are acceptable models of the linearized dynamic model of the full-scale yacht. The Jib valid models represent the largest set of linear models estimated, and each of the 102 models represents a unique trim value. 80 of these models are Type A models, and 22 are type B models. It may be theorized that the trim values are the reason for the difference between the Type A models and Type B models, as will be shown in this section. First, eigenvector modal analysis is performed for the large data set containing all 102 models in Section 8.4.1, followed by a more detailed uncertainty analysis of the best fitting 5 models of the set in Section 8.4.2, and concluding with the full details of the best estimated Jib model in Section 8.4.3.

\subsubsection{Modal Analysis for Estimated Jib Models}

In order to compare models among one another, the modes (roots of the A matrix) are calculated, separated by natural frequencies, and plotted on the complex plane. In the following 
several graphs, the modes have also been categorized according to the trim values for which each model has been computed. The roots have been color coded according to total boat velocity, roll angle, apparent wind speed, and apparent wind direction, as it has been determined these values have the largest impact on overall system dynamics. First to be analyzed are the 80 Type A models. Figure 37 shows all Type A model modes plotted in the complex plane before they are sorted according to natural frequency. The Type A models correspond to a range of trimmed apparent wind speeds from $5.3 \mathrm{~m} / \mathrm{s}(11.9 \mathrm{mph})$ to $8.1 \mathrm{~m} / \mathrm{s}(18 \mathrm{mph})$ and trimmed apparent wind angles from 41 to 70 degrees.

Each identified mode analyzed was plotted in four colors corresponding to the different system variables of trimmed total boat velocity, $V_{B}$, trimmed roll angle, $\phi$, trimmed apparent wind speed, $V_{A}$, and trimmed apparent wind angle. Not every one of these plots reveals relevant information, so only the modal plots which reveal relevant information are shown in the main body of this document, remaining plots may be found in Appendix D.

After looking at the in-depth eigenvector modal analysis, it was determined that for the eigenvector analysis, it was more appropriate to look at models which exhibited a better overall fit to the ID data, with an $R^{2}>0.88$. This reduction in data is seen in Figure 38 , and it is obvious that this additional criterion removes much of the scatter seen in the modes. The reduced data set is only used for the eigenvector modal analysis.

Lastly, for ease of discussion, the complex plane has been divided into three regions where roots may be located as shown in Figure 39. These regions are important for discussion of future control system design using the information provided in the following modal analyses. It should be noted that henceforth, stability is taken to mean dynamic stability, which is different than static stability associated with metacentric height and righting moment which is commonly referred to in naval architecture. Roots with a real component of $\sigma \leq-0.04$ are defined to be in the green Stable (S) Region, roots with a real component of $\sigma \geq 0$ are defined to be in the red Unstable (US) Region, and those roots which fall in between, ie. $-0.04<\sigma<0$, are defined to be in the Marginally Stable (MS) Region. The time constant associated with -0.04 is 25 seconds, which is long enough that a human helmsman or computer controller have enough time to prevent divergence. There is a fundamental difference between the stable and unstable models, and each would require a different robust controller. A robust controller may be used to 
directionally stabilize models which fall in the US region, and would also control models in the MS region. It is unlikely that real unstable roll behavior would actually be realized as the water has an incredible damping effect on the rolling motion of a yacht. Included in this subsection is a criteria in terms of trimmed roll angle by which these controllers should be scheduled. 


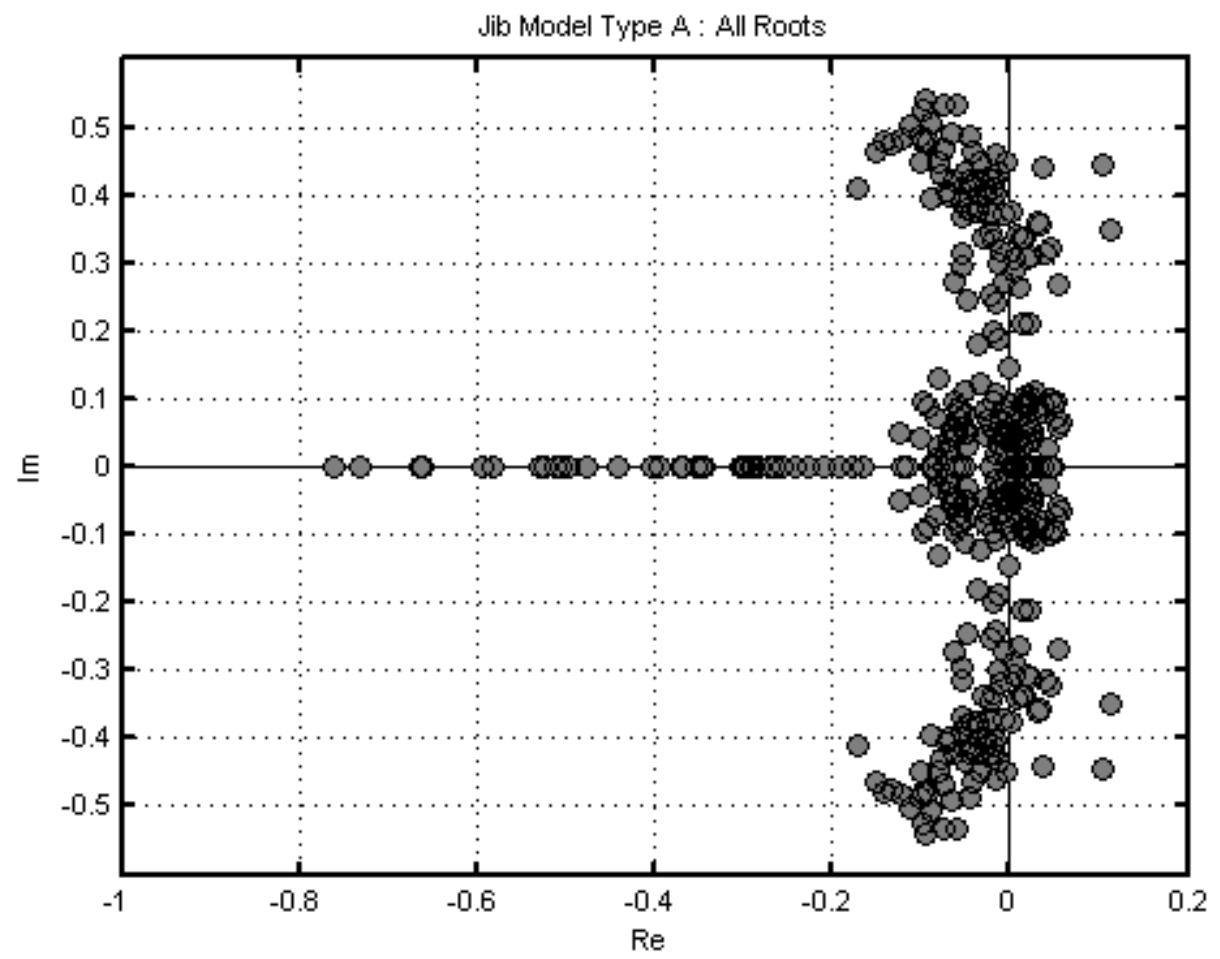

Figure 37: All Jib Type A Modes plotted on the complex plane before being sorted by natural frequency.

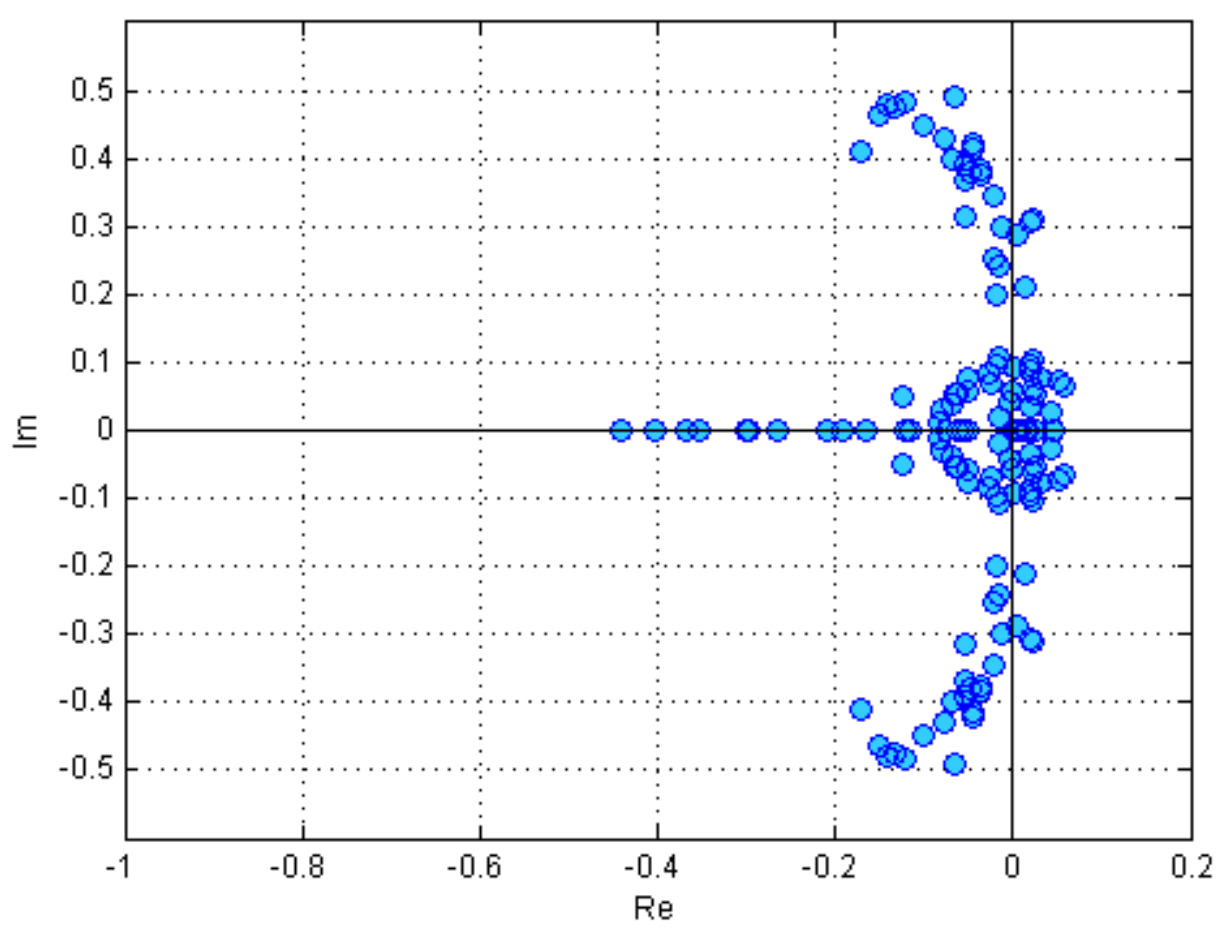

Figure 38: Jib Type A Modes for models with an $\boldsymbol{R}^{2}>0.88$ fit to the ID data which will be utilized for detailed eigenvector modal analysis. 
Recall from the previous section, Type A models exhibit 2 second order modes, labeled Mode 1A.S and Mode 2A.S, and 1 first order mode labeled Mode 3A.F. First the two second order modes will be discussed. Figure 40 is a plot of all the Modes 1A.S from the Jib estimated models colored according to trimmed roll angle. It may be seen that the range of trimmed roll angles goes from 6.9 degrees to 14.9 degrees, and that there is a clear trend with the higher roll angles clustering to the outer left of the complex plane (the lighter colored dots), and as the modes move towards the right the dots appear darker and darker, corresponding to lower and lower roll angles. Eventually they cross into the right hand side of the complex, indicating unstable modes. As Mode 1A.S moves closer to the US region, the points become more widely scattered, which shows an increase in the uncertainty in the model. This scatter should not be confused as measurement error; rather, it should be viewed as an intrinsic system characteristic. The closer a mode is to the origin of the complex plane, the less overall energy that mode contains. The lower the system energy, the more external disturbances to the system become more prevalent in overall system behavior, and the scattered modes are expected. This is not necessarily a bad characteristic, it is simply part of the system, and knowledge of this characteristic is necessary for control system design.
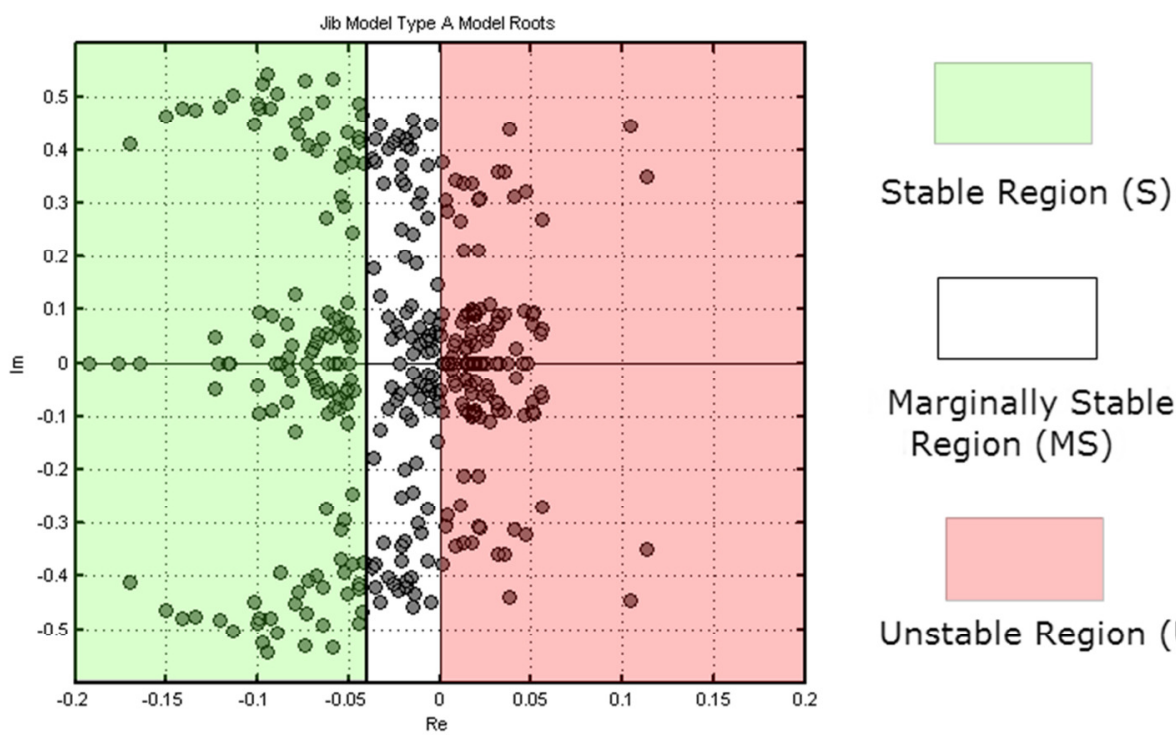

Marginally Stable Region (MS)

Figure 39: Definition of stability regions in the complex plane. 
After observing trends in similar graphs of Mode 1A.S colored according to the other three variables (found in Appendix D), it was determined that the trimmed roll angle was the defining variable for Mode 1A.S, which is supported by the eigenvector analysis. Figure 41 shows each individual states' percentage of contribution to Mode 1A.S, as defined in Eq. (8.12), plotted as a function of model trimmed roll angle. The solid blue points represent Modes 1A.S which are in the US region, the light blue points represent modes which are in the MS region, and the uncolored points represent modes in the $\mathrm{S}$ region. The red line represents a quadratic regression fit to the data. A quadratic fit was chosen because in all cases the goodness of fit was better than that of a linear regression fit.

There are five states in this model, so if each state contributed equally to the mode, each would contribute $20 \%$. Thus, any contribution in Figure 41 where the red line is above the line 0.2 , the state may be considered a significant contributor to the mode. Close examination of Figure 41 reveals this transition occurs between 8 and 10 degrees of roll for every state except yaw, where the transition happens between 12 and 14 degrees of roll. It may be concluded that

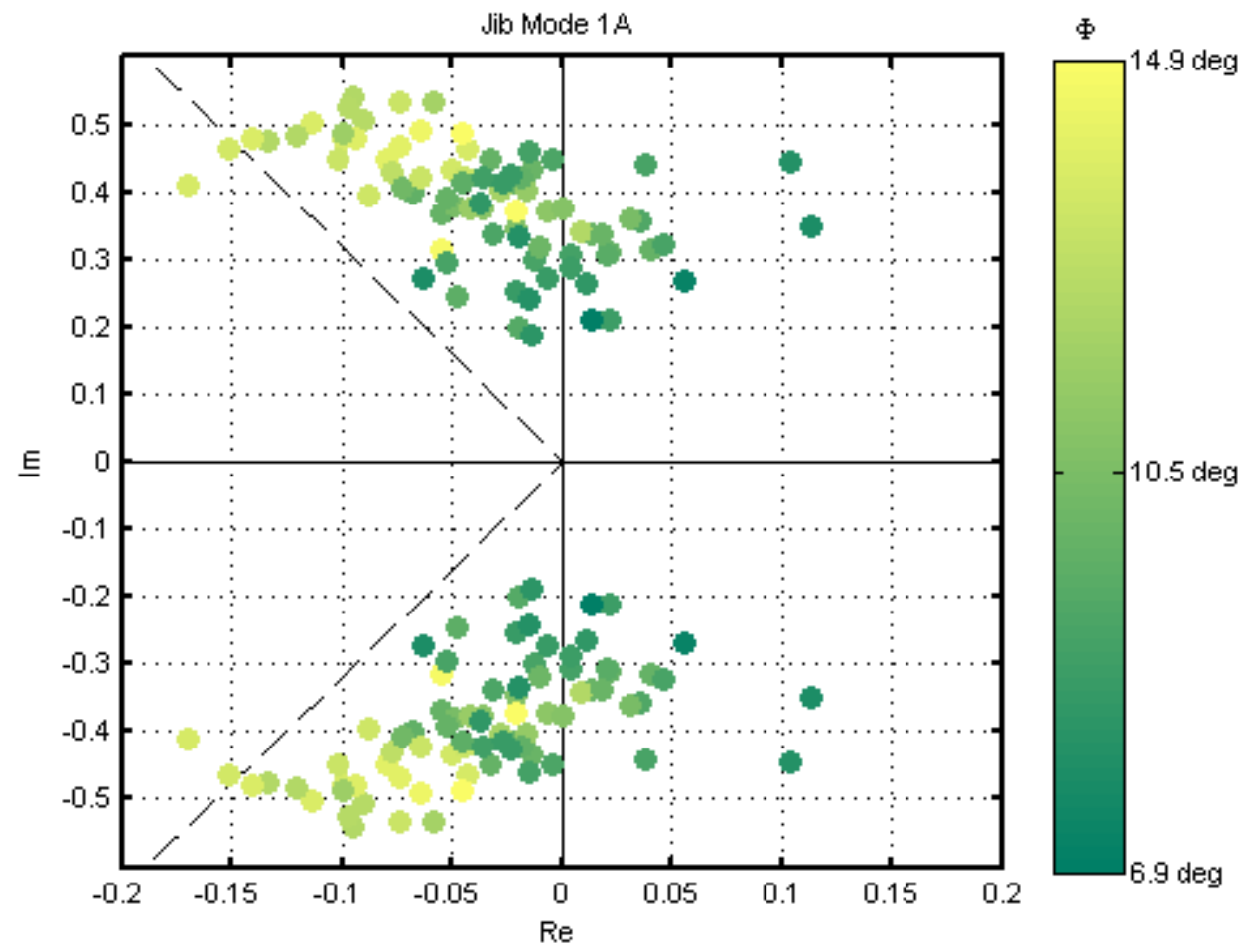

Figure 40: Jib Modes 1A showing a clear trend that this mode is dependent upon roll angle. 
for roll angles less than 8 degrees, the surge velocity and yaw rate are the motion variables which are driving the modal behavior of Mode 1A.S. It may also be concluded that above 10 degrees of roll, that roll angle and roll rate become the dominant motion variables in terms of the modal behavior of Mode 1A.S. It makes sense that the surge velocity is dominant when the boat is in a more upright (low roll) attitude, and that the roll rate is more dominant when the boat is in a more heeled (higher roll) configuration. The more heeled a sailing yacht hull is, the more sensitive it becomes to further changes in heel. Physically this is likely related to the lateral position of the aerodynamic center of the sail plan. The further the yacht heels, the faster the lateral position of the aerodynamic center changes as a function of distance from the upright position.

It was previously mentioned that a different robust controller needs designed for modes falling in the US region, and that the same controller may be used when the modes fall into the MS region. All Modes 1A.S in the reduced data set in Figure 41 which fall in either the US or
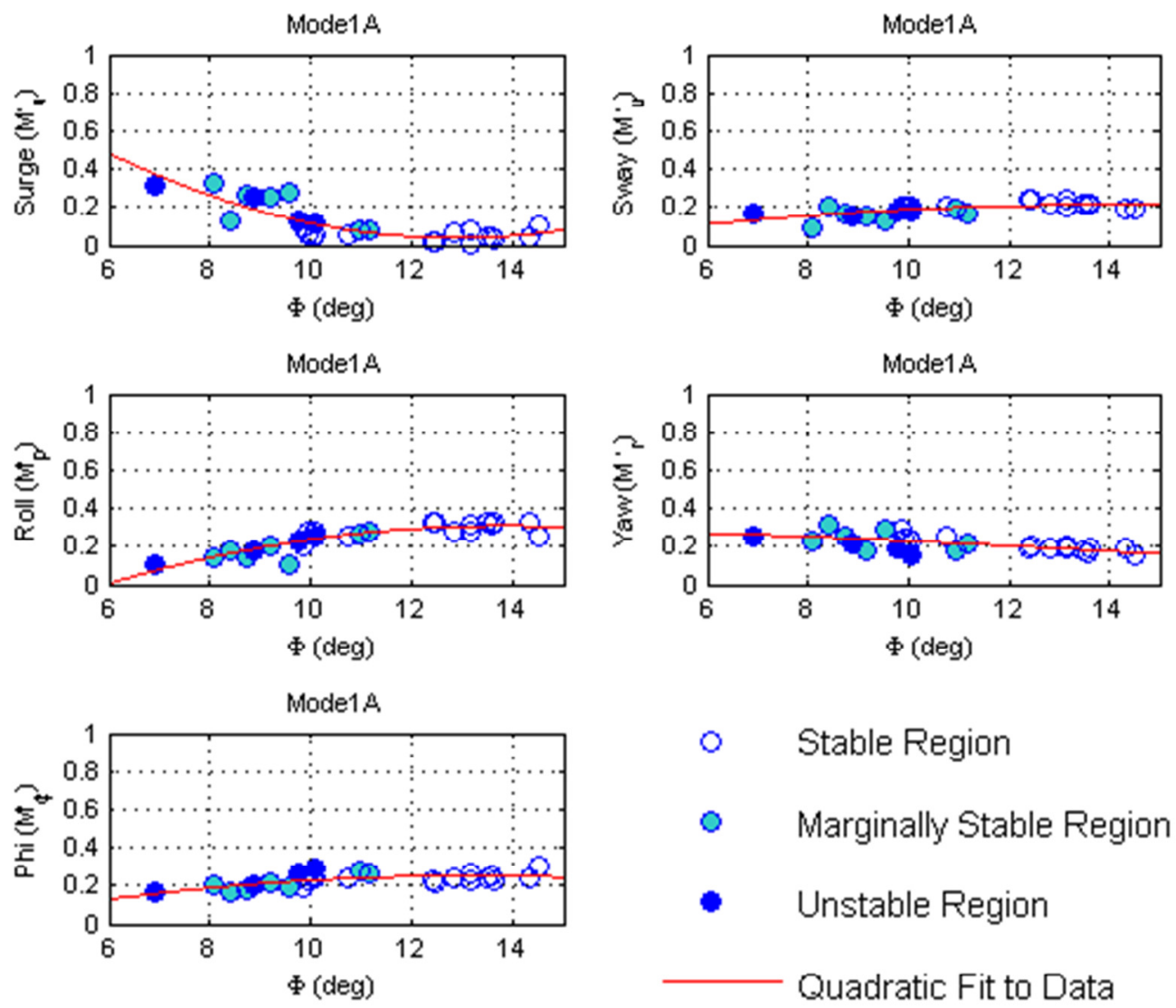

Figure 41: Contribution of State Variables to Mode 1A according to trimmed roll angle. 
MS stable region do so at trimmed roll angles less than 12 degrees. Therefore, for this particular yacht operating at a trimmed roll angle greater than 12 degrees in the Jib configuration, Mode 1A.S is expected to be dynamically stable. The region between 8 and 12 degrees of heel is a transition region where Mode 1A.S goes from being unstable at the lower range to being stable in the upper range. Since the major contributor to the mode in the low roll range is surge velocity and yaw, this instability is likely to demonstrate itself as directional instability rather than roll instability. As long as the boat is moving forward at a velocity such that the rudder is effective, a controller would not have any trouble stabilizing the system.

Next, Jib Modes 2A.S are analyzed. Figure 42 plots Modes 2A.S colored again according to trimmed roll angle. Note that Figure 42 full data set of 80 models with the lower fit criteria. This mode exhibits substantially lower damping, and therefore has a greater susceptibility to external disturbances. The highest roll angles are clustered around the origin, but the trend as far as roll is not as clear as with Mode 1A.S. Table 7 shows that the average magnitude of Mode $1 \mathrm{~A} . \mathrm{S}$ is $21 \%$ higher than that of Mode 2A.S.

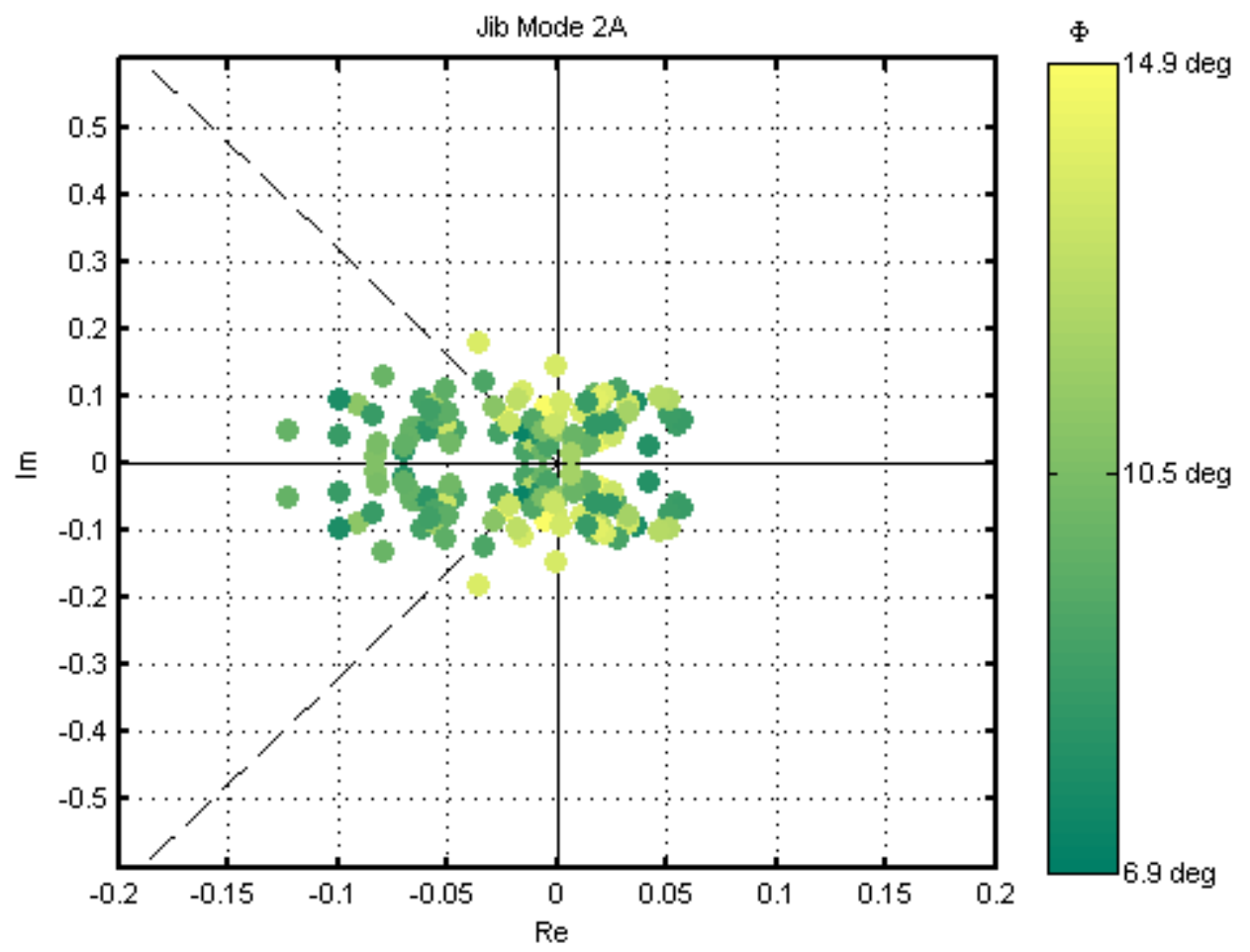

Figure 42: Second order Modes 2A colored according to trimmed roll angle. 
Table 7: Difference in the average magnitude of Modes 1A and Modes 2A

\begin{tabular}{|c|c|c|}
\hline $\begin{array}{c}\text { Ave. Magnitude of Mode } \\
1 \mathrm{~A}\end{array}$ & $\begin{array}{c}\text { Ave Magnitude of Mode } \\
2 \mathrm{~A}\end{array}$ & \% Difference \\
\hline 0.38 & 0.08 & $21 \%$ \\
\hline
\end{tabular}
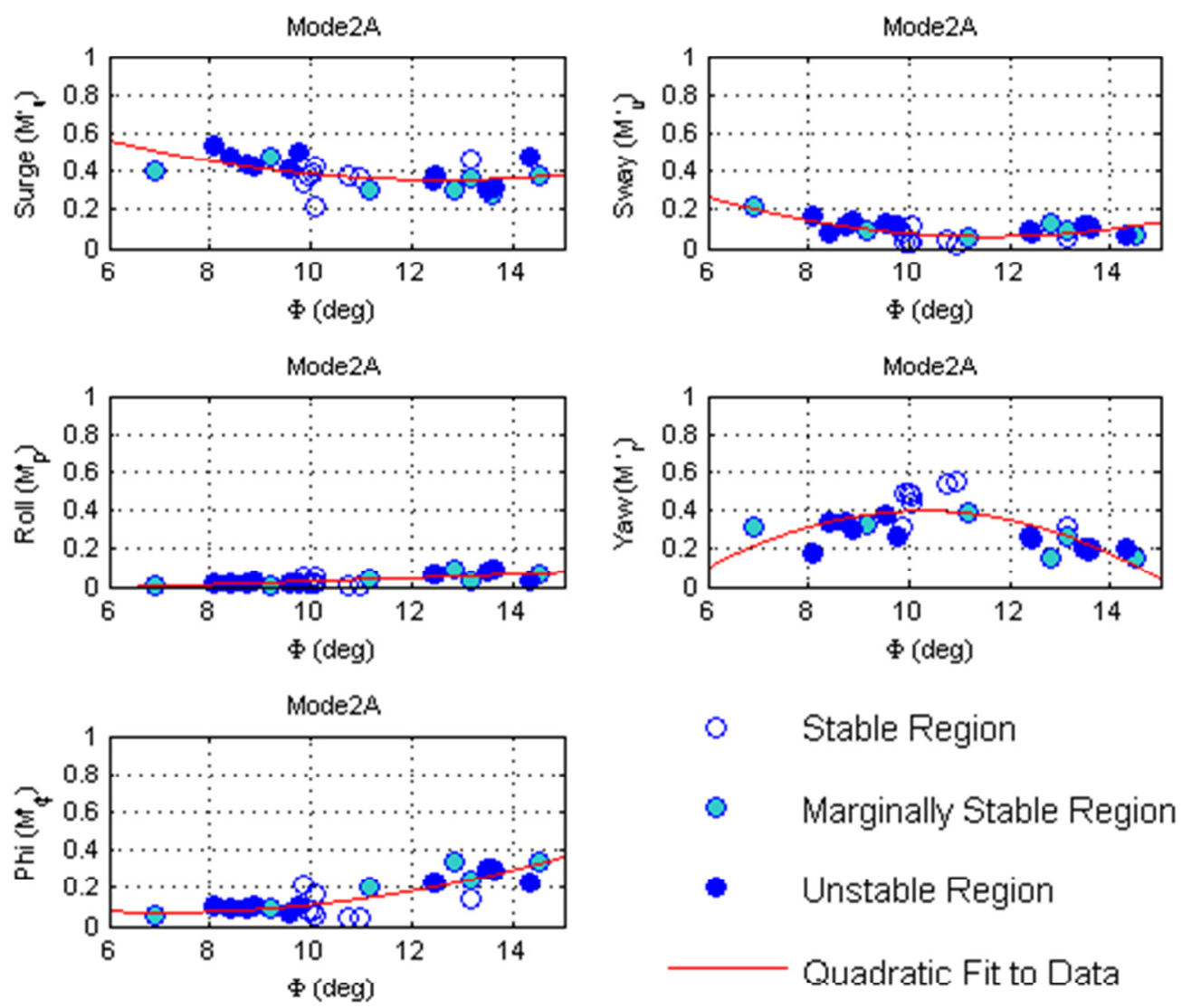

Figure 43: Contributions of each state to Mode 2A according to trimmed roll angle. 
The eigenvector analysis of Mode 2A.S reveals that surge is the greatest contributor to this mode through the entire range of roll angles as shown in Figure 43, followed closely by yaw rate. The unstable and marginally stable models are distributed throughout the roll range. It is difficult to make conclusions about this mode because it has such small magnitude. The dashed lines in Figure 40 and Figure 42 represent lines of constant damping ratio, $\zeta=0.3$. From these dashed lines to the real axis represent the range $\zeta<0.3$. According to (Ogata, 1997), any modes that have damping less than 0.3 may be considered low damped modes, and therefore considered low energy modes. As may be seen in Figure 40, most of the data points for Mode 1A.S fall above this range, but just slightly. All the points for Mode 2A.S shown in Figure 42 fall below this range, indicating a very low energy mode which means it is very susceptible to external disturbances. The fact that this is a low energy mode also means it is a difficult mode to excite, and therefore will be difficult to characterize. In the low roll angle region Mode 2A.S overlaps with Mode 1A.S in terms of surge and yaw rate being the highest contributors to the mode.

There are two primary sources of uncertainty that may be contributing to the difficulty in isolating this mode: the original data smoothing may have lost key information and designed system inputs were not appropriate for exciting this mode's dynamics. The first difficulty can be addressed with a different filtering strategy for the data. The second difficulty, which is likely the more prevalent, may be addressed by using the observed natural frequency of identified modes and using a well-known rule of thumb (Jategaonkar, 2006), shown in Eq. (8.14), to design future inputs.

$$
\Delta t_{D B L T} \approx \frac{2.3}{\omega_{n}}
$$

where $\Delta t_{D B L T}$ is the half period in seconds of a doublet input. The average natural frequency of Mode 2A.S is $0.08 / \mathrm{s}$. According to the rule of thumb, this would mean the each side of the doublet input would have to be held for 29 seconds. This is probably impractical for the sailing yacht, as even one or two degrees of rudder held away from trim for 29 seconds is going to take the heading very far from the trimmed heading and apparent wind angle. Using a multi-step input which considers the energy in the mode as described in (Jategaonkar, 2006) may be necessary. 

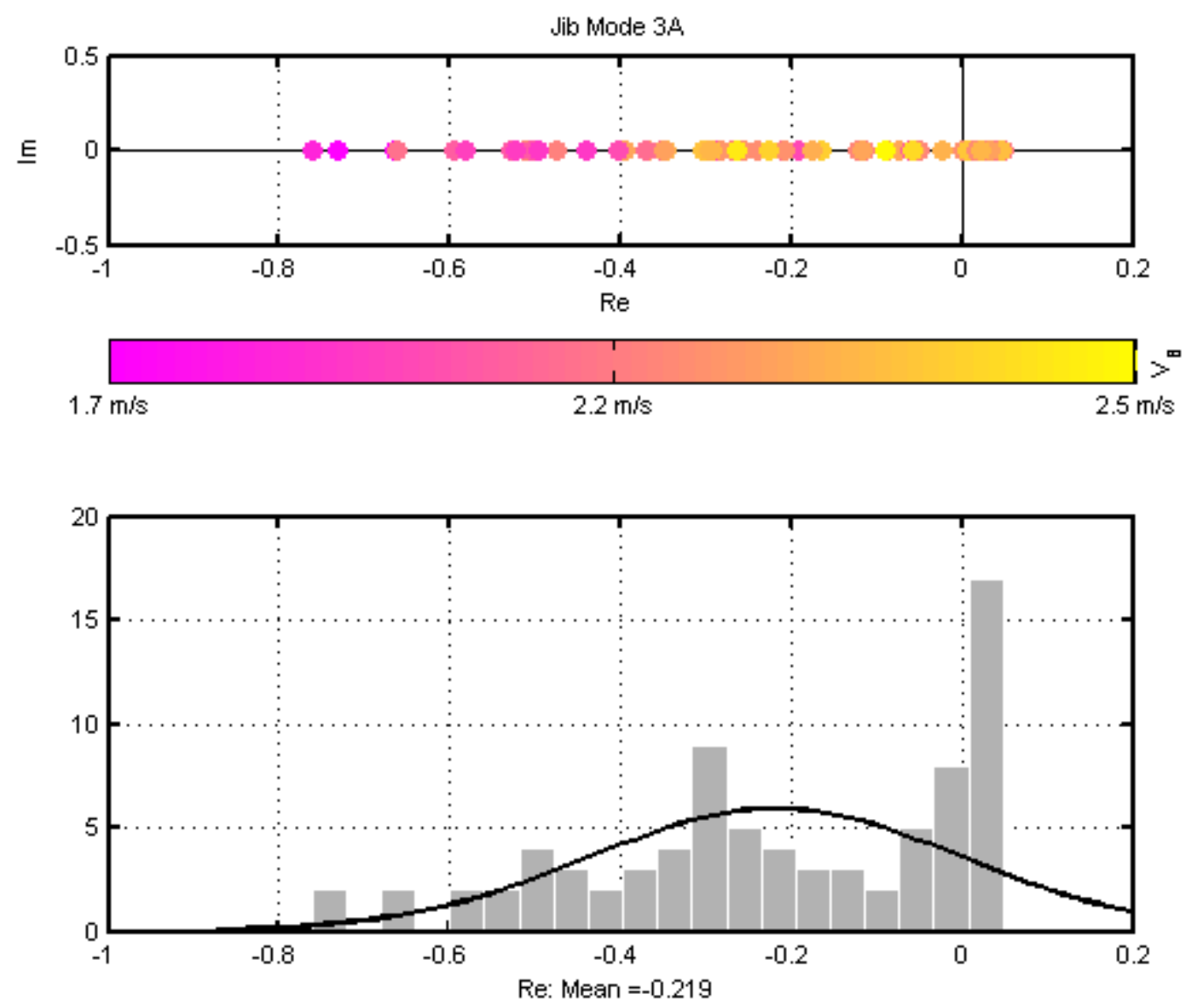

Figure 44: Modes 3A according to trimmed boat velocity.

.Last to be analyzed the first order Mode3A.F. This mode shows an extremely large spread over the real axis, as shown in Figure 44, which shows the most stable models in terms of this mode correspond to slower boat speeds. The histogram shows two peaks in location of this mode, with the largest peak being in the US region to the left of the real axis. Figure 45 represents the reduced set of models with $R^{2}>0.88$, and shows the eigenvector contribution of each mode with respect to trimmed surge velocity, $U$. There are no data points in the MS region for the reduced data set. Most of the stable models see around a 20\% contribution from surge, somewhere between $20 \%$ and $30 \%$ contribution from sway (this is the only mode sway in which sway is significant), and around a $40 \%$ contribution from yaw rate. The major difference between the unstable and stable modes here is all unstable modes have a surge contribution greater than $40 \%$, and for such cases a controller would need to be designed for the unstable modes. 

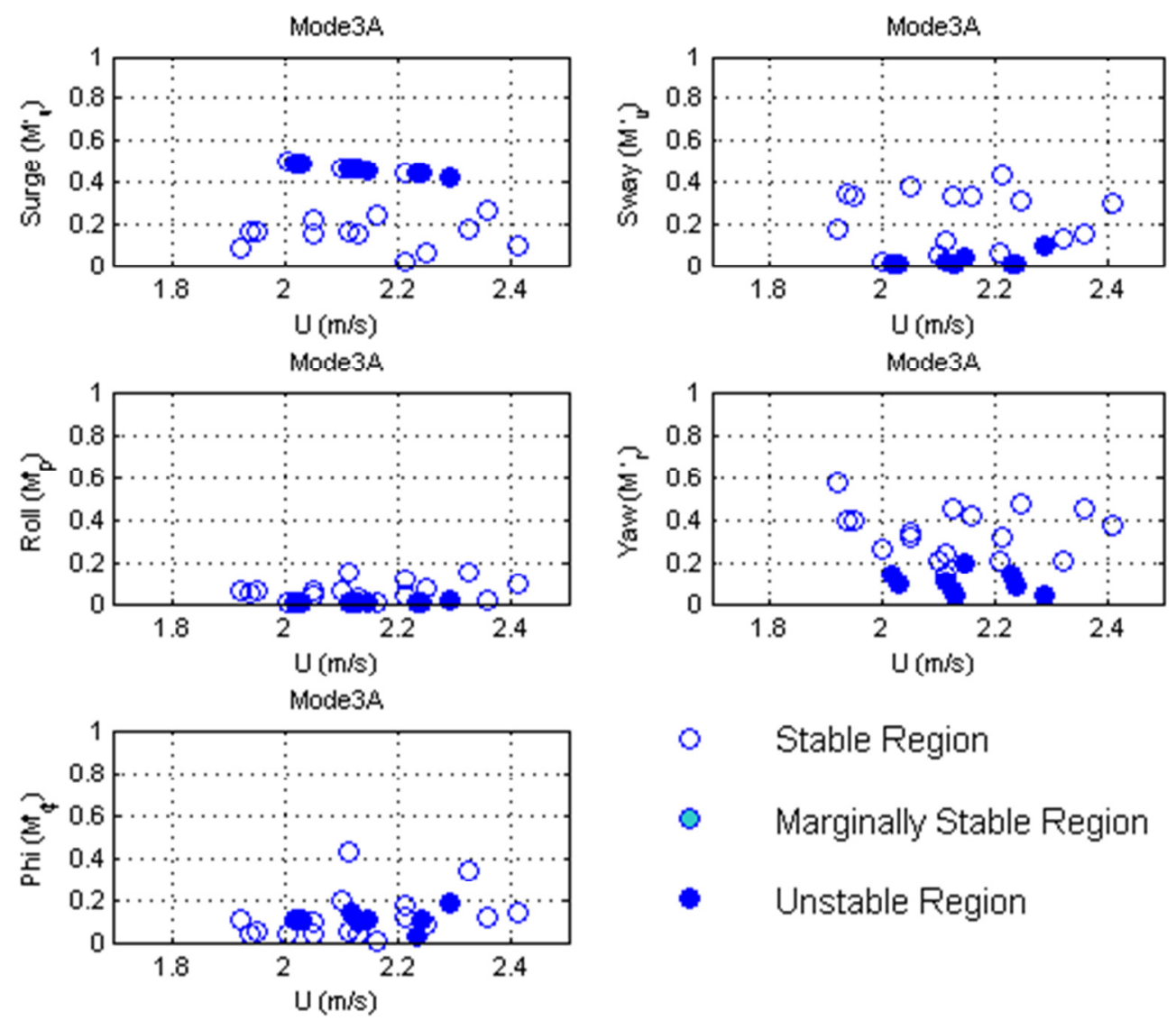

- Stable Region

- Marginally Stable Region

- Unstable Region

Figure 45: Contributions of each state to Mode 3A with respect to $U$.

That concludes the analysis for the Jib Type A Modes, moving on to the analysis of the Type B models, of which there are significantly fewer than Type A models, Modes 1B, 2B, 3B, and 4B may be examined. First, Mode 4B.S, the second order mode, will be analyzed. Figure 46 shows Mode 4B.S colored according to apparent wind angle, with all the modes lying above the low damping line meaning this is the second order mode that corresponds to Mode $1 \mathrm{~A} . \mathrm{S}$ of the Type A models. There is not as clear a trend with roll angle in Figure 46 as there was in Figure 40. However, the eigenvector analysis in Figure 47 where the data has been reduced again to models with $R^{2}>0.88$, shows a similar trend as was seen in Figure 41 for Mode 1A.S. Figure 47 supports the conclusion that above 10 degrees of roll, roll rate and roll angle are the dominant motion variables for this mode of motion. It also supports the conclusion that the region from 812 degrees of roll is the transition region between stability and instability in this mode. 


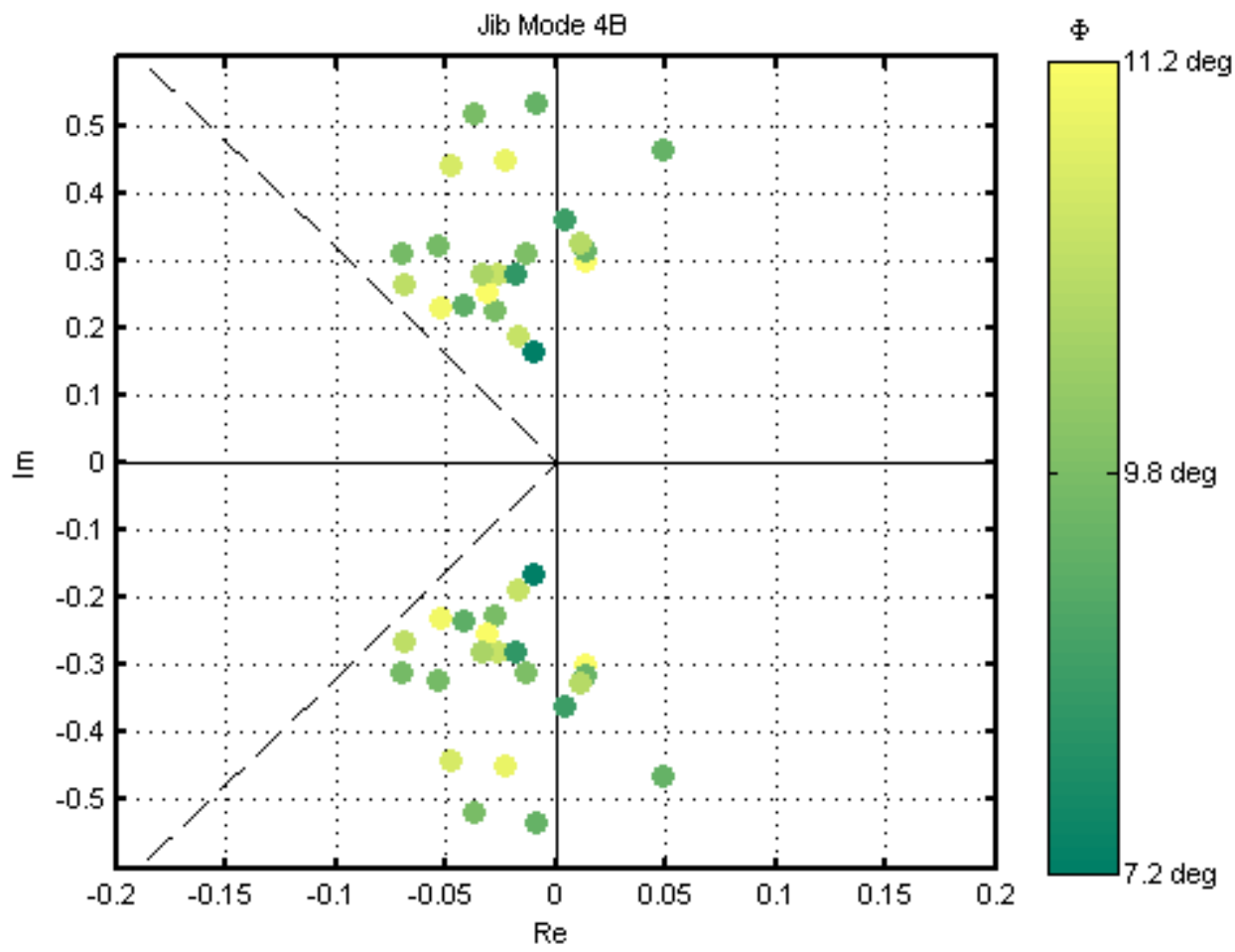

Figure 46: Mode 4B colored according to trimmed roll angle.
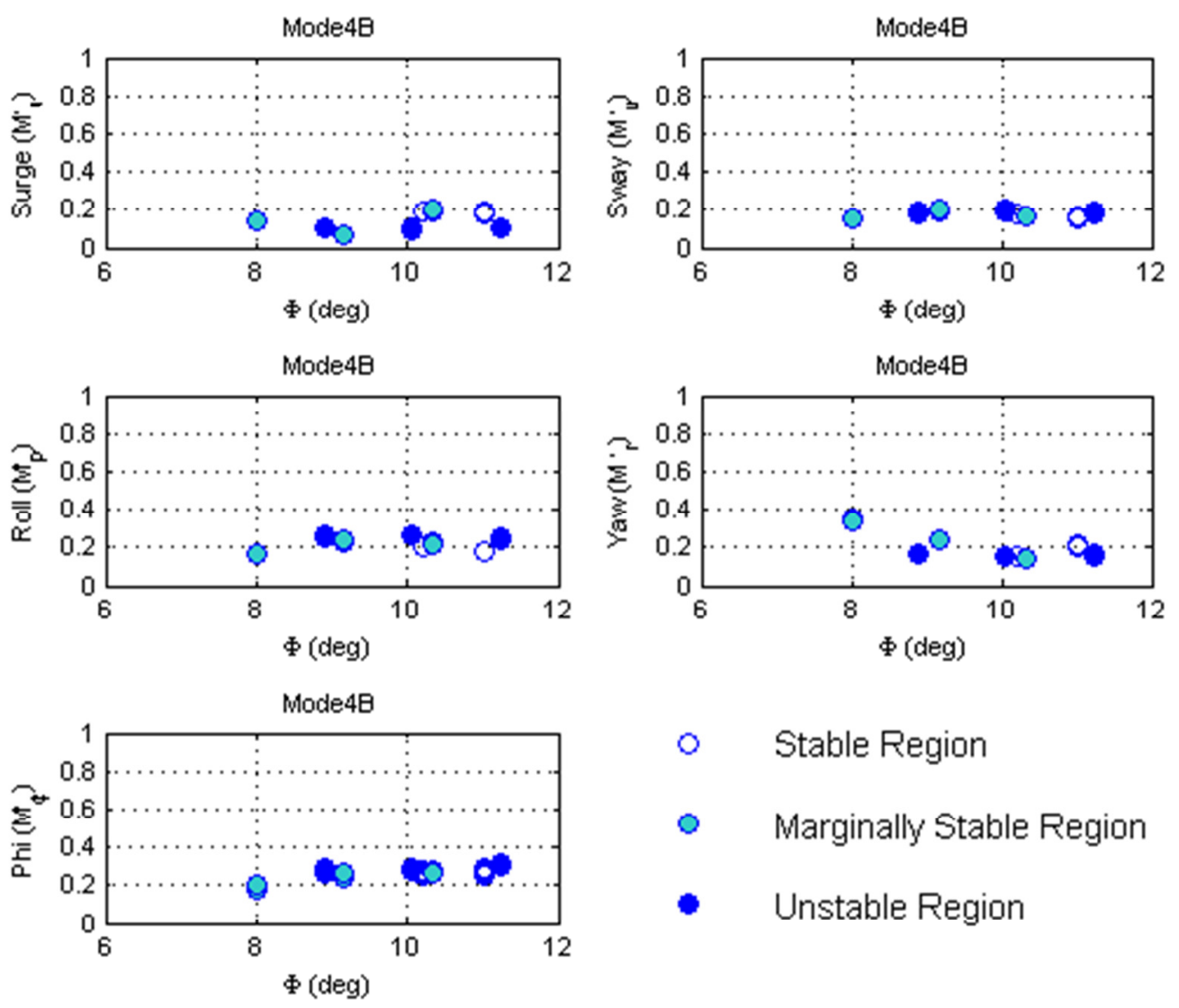

- Stable Region

- Marginally Stable Region

- Unstable Region

Figure 47: Contribution of each state to Mode 4B according to trimmed roll angle. 
Next, the analysis of the three first order modes from the Type B models is performed. Mode 1B.F corresponds to Mode 3A.F and is plotted on the real axis colored according to trimmed boat speed in Figure 48. As in Figure 44, the points farthest left on the real axis correspond to low trimmed boat speeds. The histogram of Figure 48 shows the spread of the modes along the real axis. This mode lies completely in the $\mathrm{S}$ region. The eigenvector analysis of Mode 1B.F in Figure 49 shows that yaw rate is the largest contributor to this mode.

Figure 51 shows Mode 2B.F colored according to trimmed apparent wind angle. The histogram shows that this mode is centered upon $\sigma=-0.051$. The eigenvector analysis for this mode in Figure 51 shows that this mode is dependent upon the trimmed surge velocity, U. This is evident where there is a sharp decline over the range of $U$ in the contribution of yaw rate to the
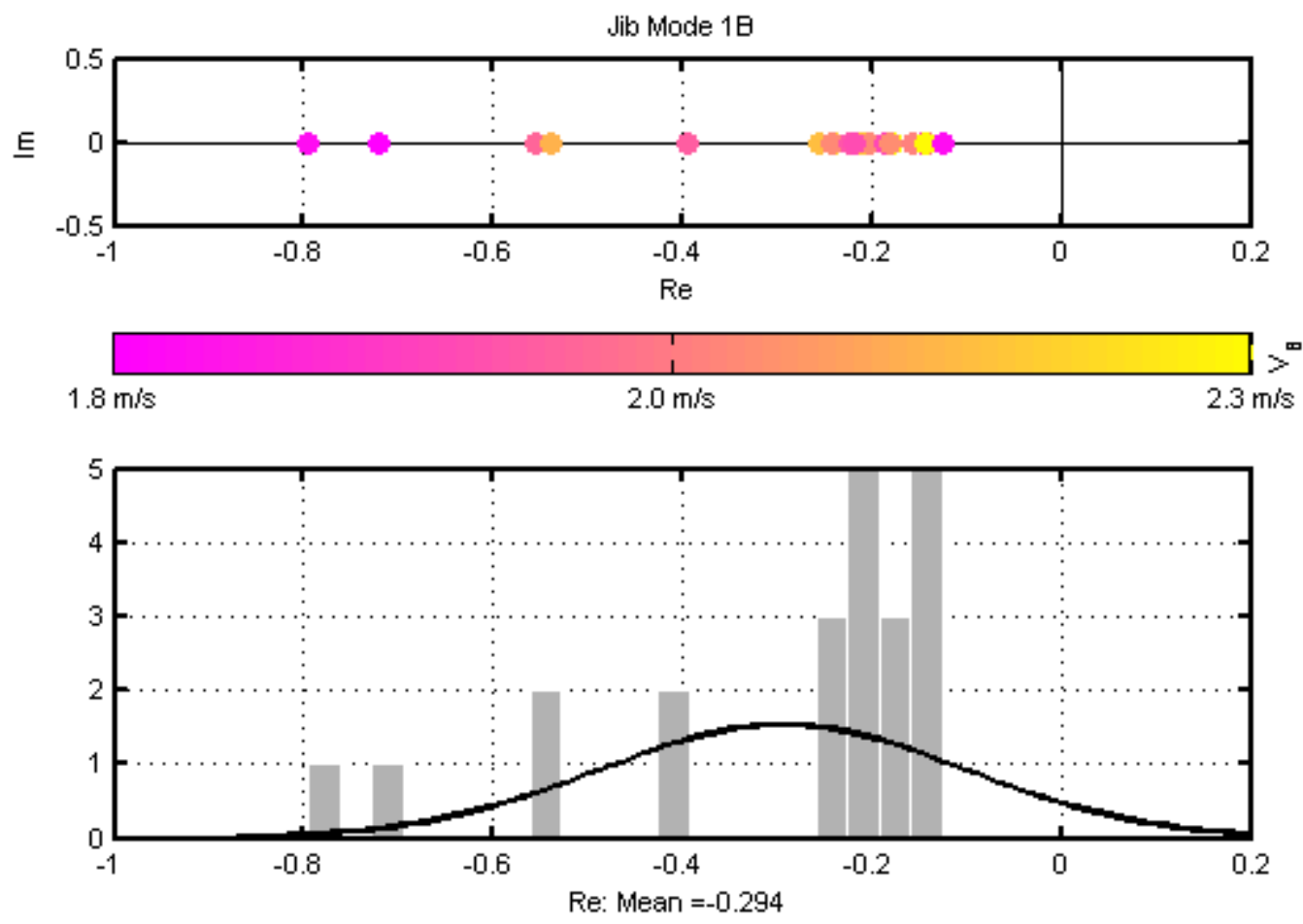

Figure 48: Modes 1B colored according to boat velocity, clearly clustered to the far left of the imaginary axis. 

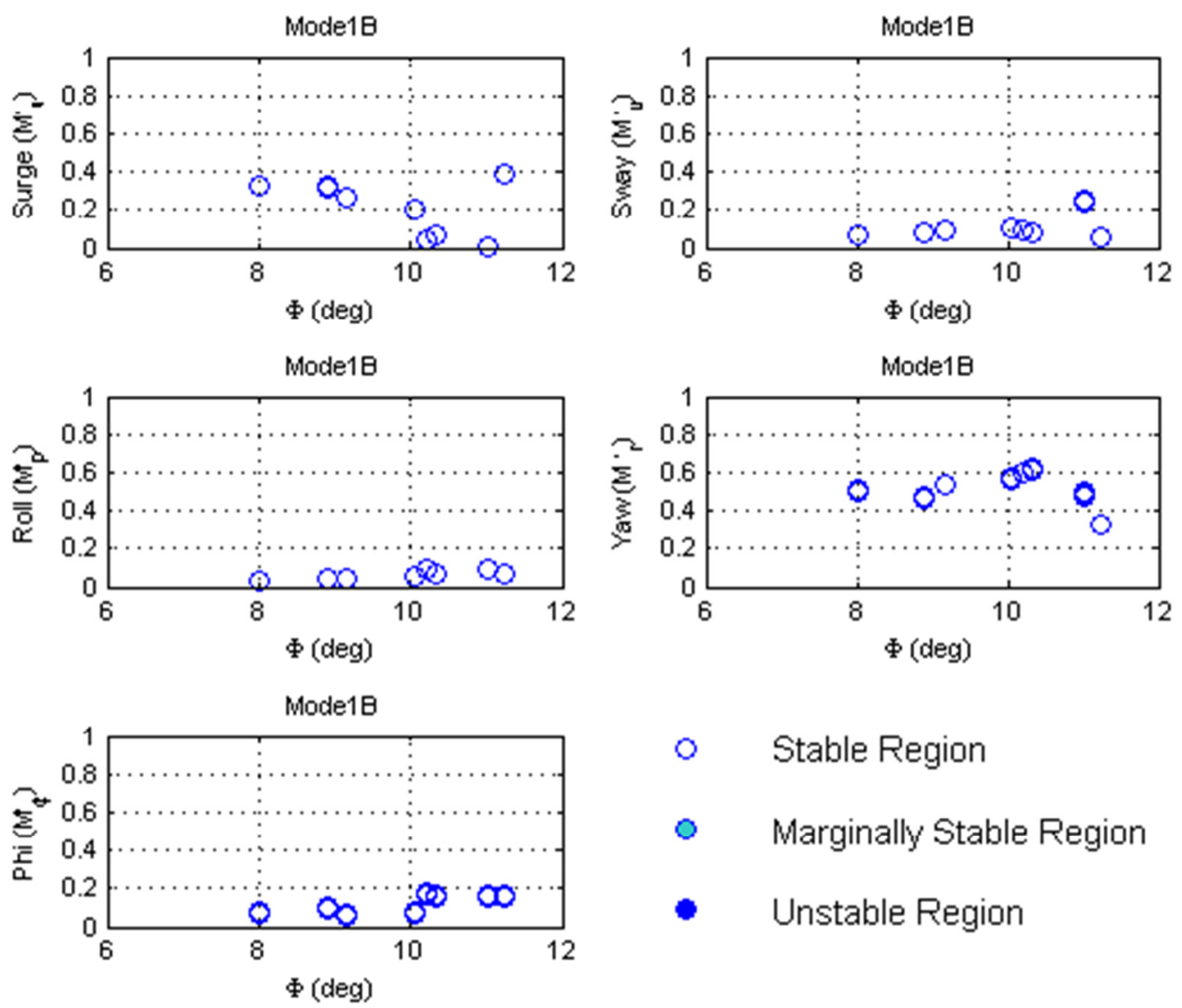

- Stable Region

- Marginally Stable Region

- Unstable Region

Figure 49: Contribution of each state to Mode 1B according to $U$.
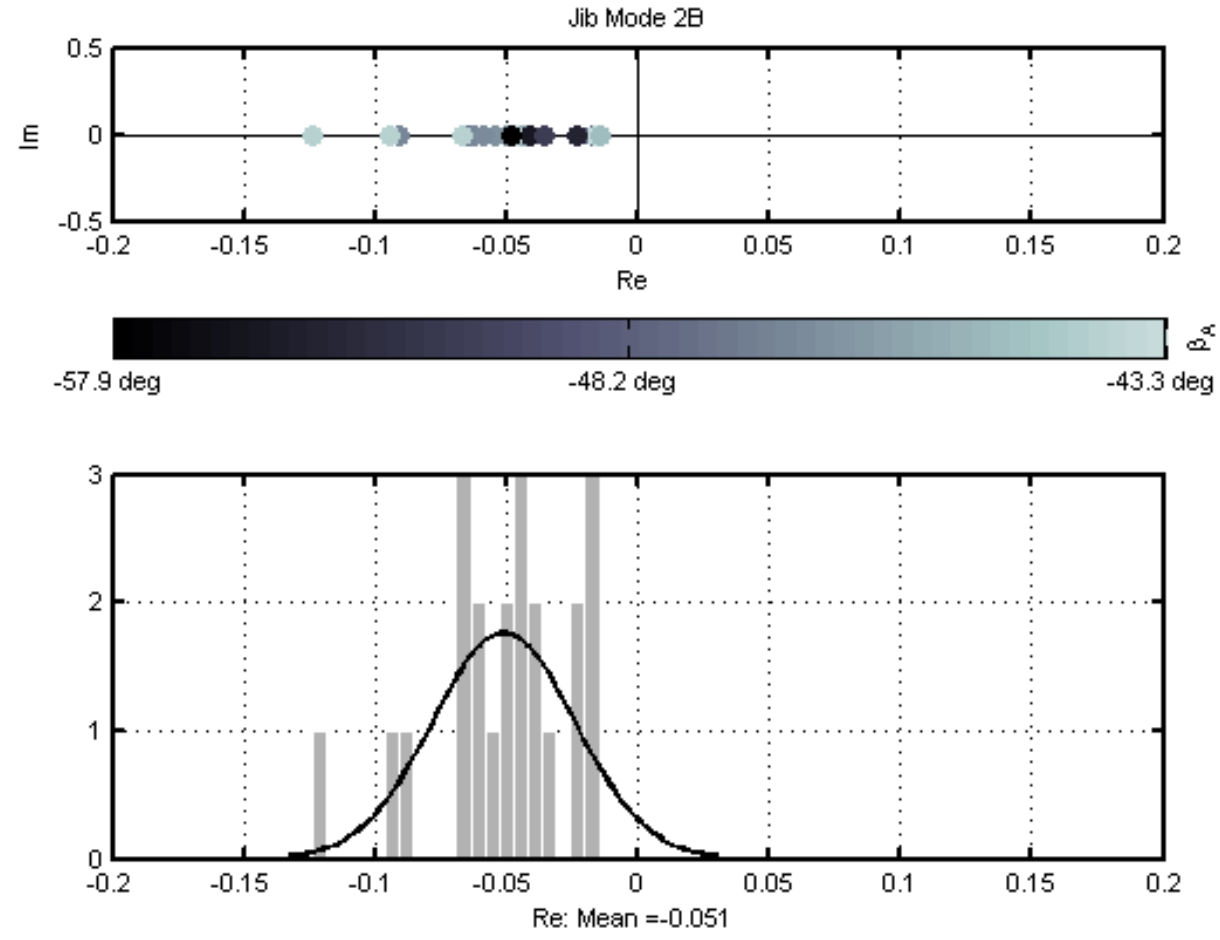

Figure 50: Mode 2B colored according to apparent wind angle. 

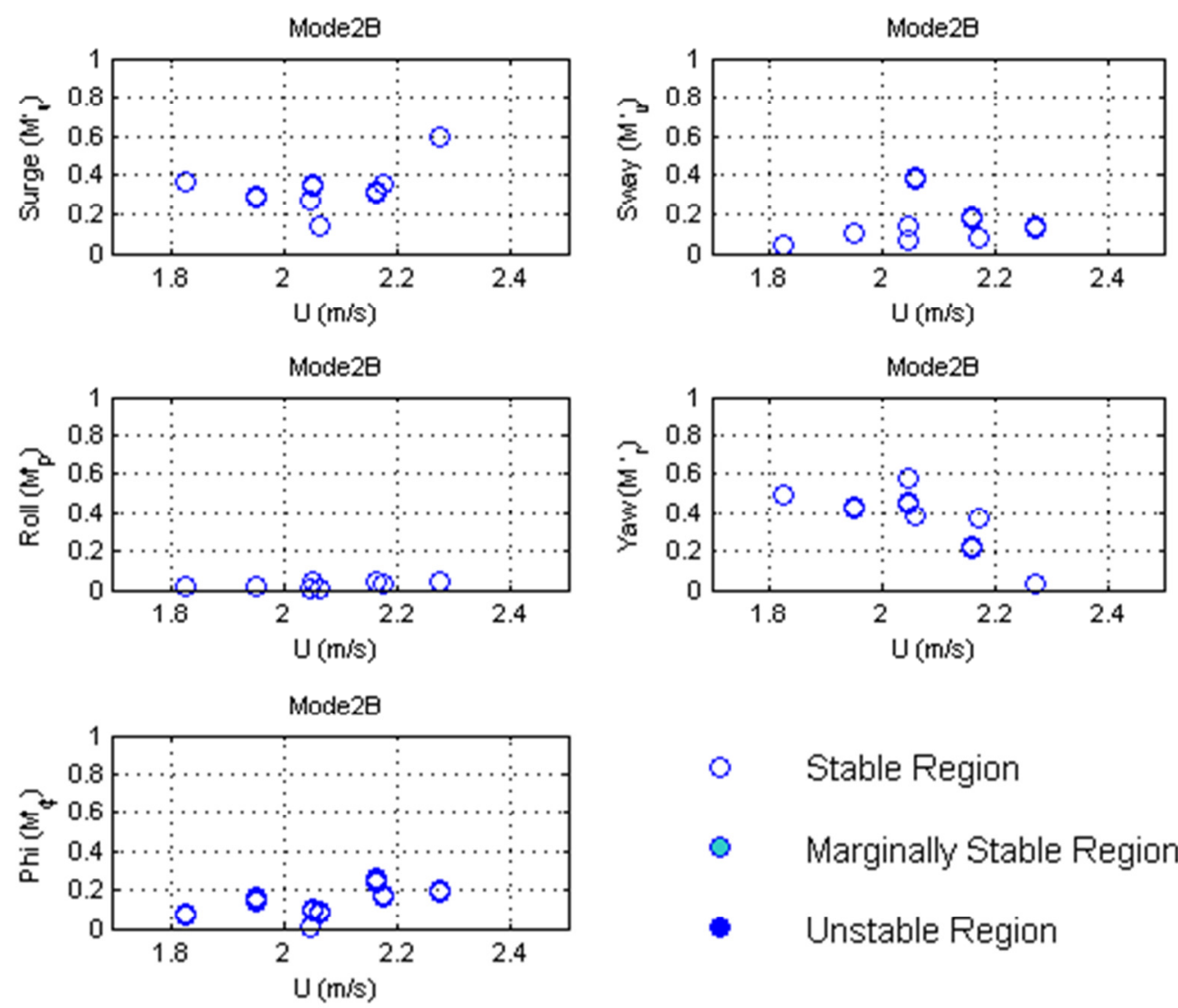

Stable Region

- Marginally Stable Region

- Unstable Region

Figure 51: Contribution of each state to Mode 2B according to $U$.

modal behavior. Regardless, this mode is stable. Figure 52 shows Mode 3BF colored according to trimmed apparent wind angle. This mode is likely to be unstable, with the histogram of Figure 52 showing the mode centered at $\sigma=0.015$. The eigenvector analysis of Mode 3B.F reveals that surge is always the largest contributor to this mode, with all models showing a contribution in surge of higher than $40 \%$. The eigenvector analysis indicates that all Modes 3B.F which correspond to trimmed surge velocities less than $2.2 \mathrm{~m} / \mathrm{s}$ fall in the US or MS region, however, further data points are needed before a more definite conclusion relating surge velocity to the stability of Mode 3B.F can be made. Modes 2B.F and 3B.F are related to Mode 2A.S of the Type A models. The Type B models result when Mode 2A.S meets the real axis and splits into two first order modes. 

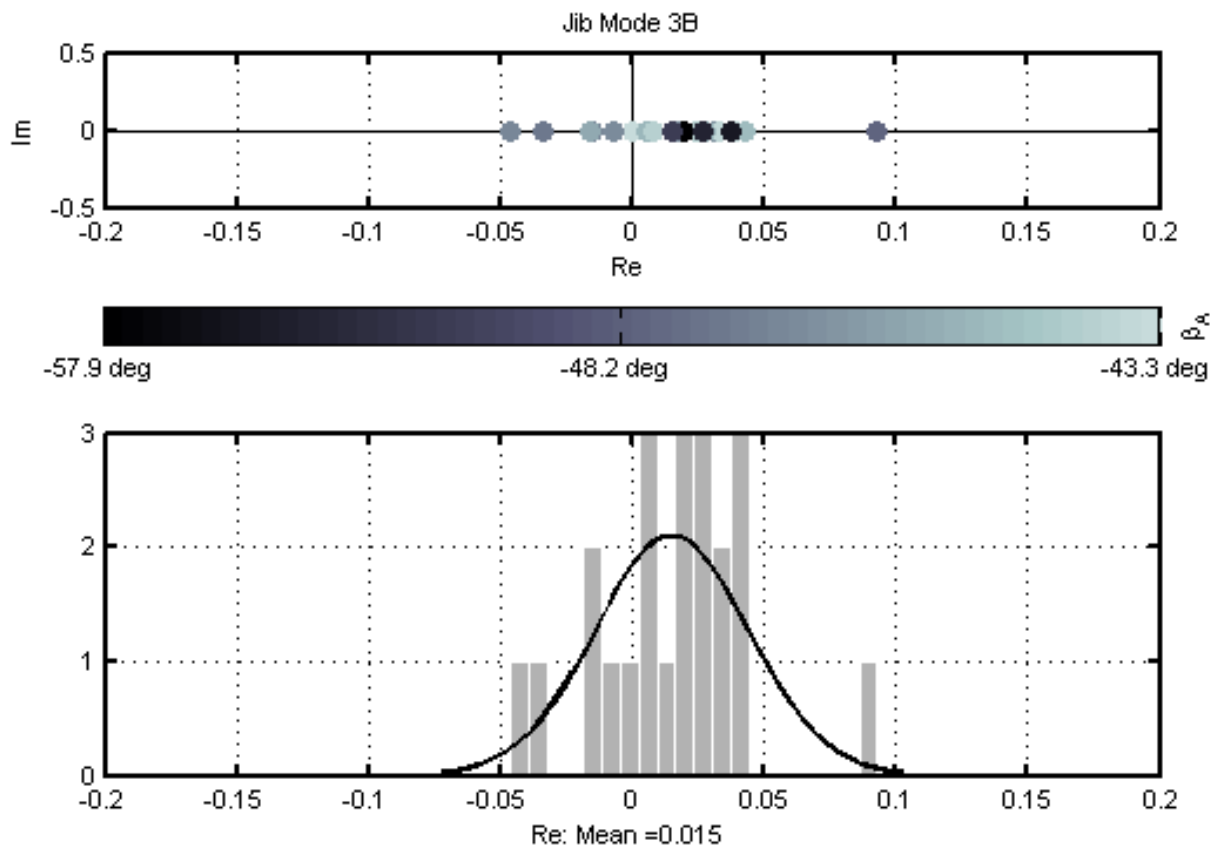

Figure 52: Mode 3B colored according to apparent wind angle.
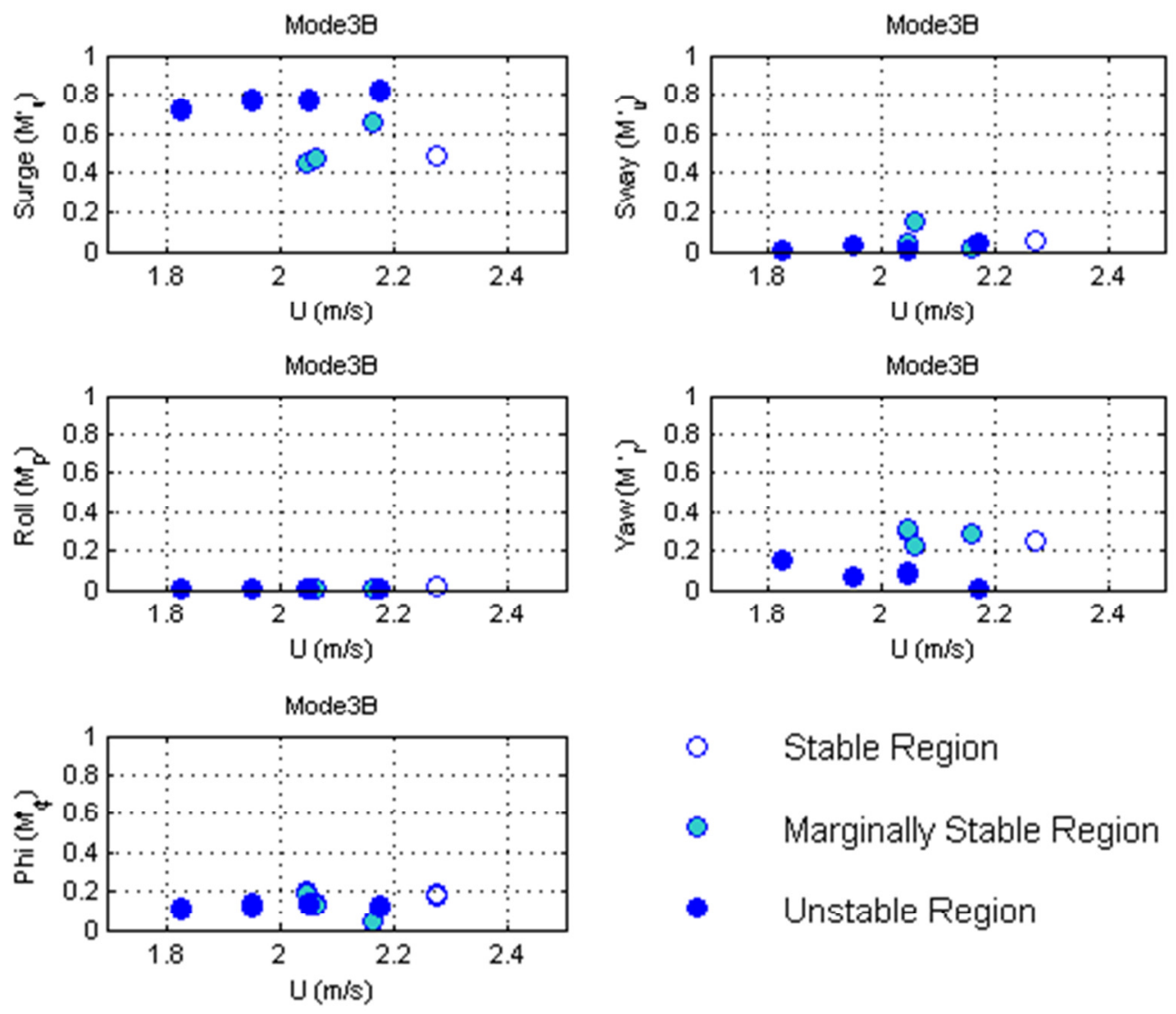

- Stable Region

- Marginally Stable Region

- Unstable Region

Figure 53: Contribution of each state to Mode 3B according to $U$. 
Now it is possible to make comparisons and analogies between the Type A and Type B models. Mode 1A,S clearly corresponds to Mode 4B.S, and Mode 3A.F clearly corresponds to Mode 1B.F. Mode 2B.F and Mode 3B.F are responsible for the difference between the Type A and Type B models. As the system is given less energy from the wind, Mode 2A.S has lower and lower damping until it splits into Mode 2B and Mode 3B of the Type B model. This is demonstrated in Table 8 and Table 9, in which the differences in the overall range of the state variables is compared between the Type A and Type B models.

Table 8: Comparison of the ranges represented by the Type A and Type B models in the Jib configuration.

\begin{tabular}{|c|c|c|c|}
\hline Model Type & Type A & Type B & $\begin{array}{c}\% \text { Difference in } \\
\text { Max }\end{array}$ \\
\hline Range for $V_{B}$ & $1.7-2.5 \mathrm{~m} / \mathrm{s}$ & $1.8-2.3 \mathrm{~m} / \mathrm{s}$ & $9 \%$ \\
\hline Range for $\phi$ & $6.9-14.9 \mathrm{deg}$ & 7.2 to $11.2 \mathrm{deg}$ & $33 \%$ \\
\hline Range for $V_{A}$ & $5.3-8.1 \mathrm{~m} / \mathrm{s}$ & $5.5-7.4 \mathrm{~m} / \mathrm{s}$ & $9 \%$ \\
\hline Range for $\beta_{A}$ & $41-70 \mathrm{deg}$ & $43-60$ deg & $17 \%$ \\
\hline
\end{tabular}

Table 9: Comparison of the mean boat velocity, roll angle, apparent wind speed and apparent wind direction for the estimated Type $A$ and Type $B$ models.

\begin{tabular}{|c|c|c|c|}
\hline Model Type & Type A & Type B & $\%$ Difference \\
\hline Mean for $V_{B}$ & $2.1 \mathrm{~m} / \mathrm{s}$ & $2.0 \mathrm{~m} / \mathrm{s}$ & $5 \%$ \\
\hline Mean for $\phi$ & $10.9 \mathrm{deg}$ & $9.5 \mathrm{deg}$ & $14.7 \%$ \\
\hline Mean for $V_{A}$ & $6.8 \mathrm{~m} / \mathrm{s}$ & $6.5 \mathrm{~m} / \mathrm{s}$ & $4.6 \%$ \\
\hline Mean for $\beta_{A}$ & $51.5 \mathrm{deg}$ & $48.3 \mathrm{deg}$ & $6.6 \%$ \\
\hline
\end{tabular}


Table 8 lists the differences in trimmed ranges for the four main variables of boat velocity, roll angle, apparent wind speed and direction. In each category, the upper limit of the ranges is higher for the Type A models. The maximum roll angle is $33 \%$ greater for the Type A models than the Type B models. To narrow down the difference between the models,

Table 9 compares the mean of the distribution of each of the motion variables. The mean roll of Type A models is $15 \%$ higher than the mean roll angles of the Type B models. In the apparent wind angles represented by this range (upwind through a close reach), it is expected that there is a relationship between roll angle and wind speed. Higher wind speeds mean more energy is being put into the system, which should also correspond to larger roll angles. In another work studying the dynamics of the tall-ship Pride of Baltimore, the relationship of apparent wind speed squared to roll angle was shown to be a significant parameter in the dynamic behavior of the ship(Johnson et al., 2009). Therefore, it is not surprising that in this analysis roll angle has proven to be a primary differentiator between the higher energy Type A models and the lower energy Type B models.

The Type A models exhibit Mode 1A.S which is far from the origin, and second order mode 2A.S which is clustered around the origin. At some point, there is a threshold where mode 2A.S becomes a first order mode and the system changes from Type A to Type B. It makes sense, then, that the Type B systems are generally of lower energy (lower wind speeds). In the higher energy Type A system, the boat is able to oscillate and exhibit the second order behavior, but in the lower energy system, the damping component of the water overcomes that second order behavior, and that vehicle then exhibits first order behavior.

The distribution of the motion variables within the range is shown in Figure 54 for the Type A models and Figure 55 for the Type B models. Figure 54 and Figure 55 show the apparent wind direction for both Type A and Type B models more models were collected and estimated towards the upwind (less negative) direction. Verification of these results in sailing trim closer to the beam reach condition should be performed in future analyses. 

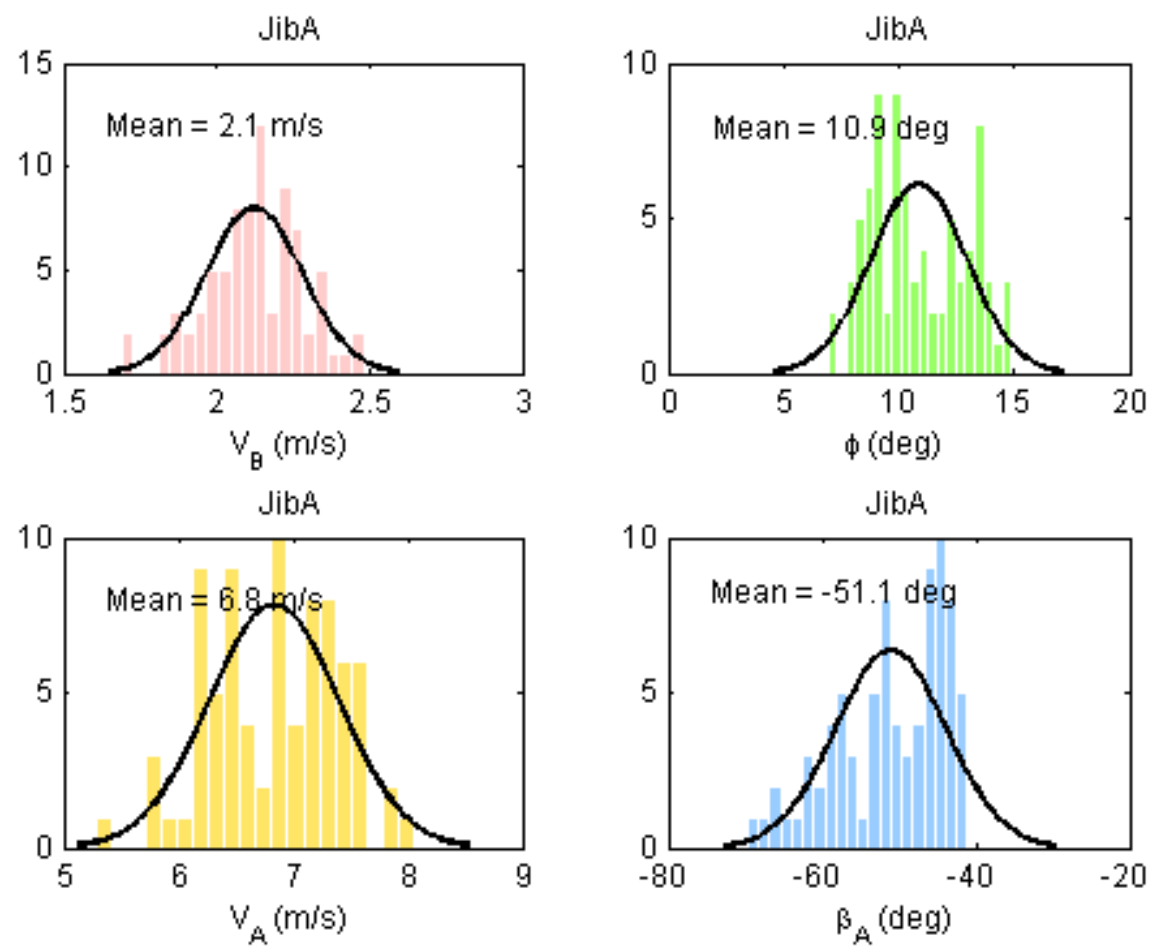

Figure 54: Distribution of trimmed boat speeds, roll angles, apparent wind speeds and angles for the estimated Type A Jib Models.
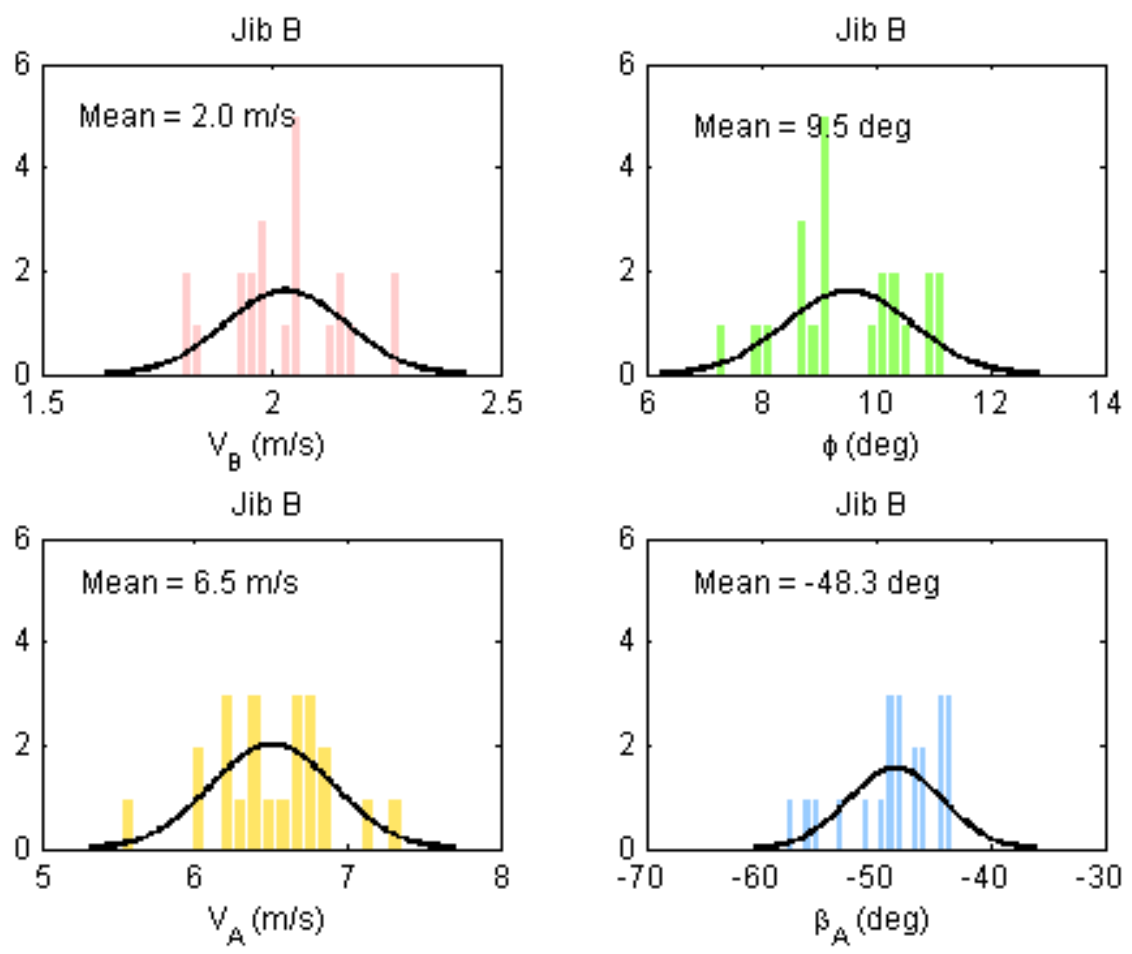

Figure 55: Distribution of trimmed boat speeds, roll angles, apparent wind speeds and angles for the estimated Type B Jib Models. 
This subsection contains the most important conclusions that may be drawn as part of this dissertation, thus, the conclusions of this section are summarized below:

1. Mode 1A.S corresponds to Mode 4B.S, and is very dependent upon trimmed roll angle. Mode 1A.S is always stable for trimmed roll angles greater than 12 degrees. For trimmed roll angles greater than 10 degrees, roll angle and roll rate dominate the modal behavior. At trimmed roll angles less than 8 degrees, surge and yaw rate dominate the modal behavior.

2. Mode 2A.S is a low energy mode with damping less than 0.3 and a magnitude $21 \%$ less than Mode 1A.S. As the system loses energy Mode 2A.S splits into Mode 2B.F and Mode 3B.F, which represents the difference between the Type A and Type B models. Mode 2A.S is not very well characterized by eigenvector analysis, likely due to inadequate excitation of such a low energy mode. Surge velocity and yaw rate dominate the modal behavior.

3. Mode 3A.F must be quantified by eigenvector analysis for control system design. If $M^{\prime}{ }_{u}<0.4$ and $M_{v}^{\prime}>0.2$, it may be considered a stable mode. If these criterion are not met the mode must be treated as unstable for control system design.

4. To do a meaningful modal analysis, models should have an $R^{2}>.88$ fit to the ID data. 


\subsubsection{Presentation of the Best Five Jib Models}

Now that all 102 of the estimated Jib models have been presented and discussed, a closer look will be taken at the 5 best model fits in the Jib configuration. The best 5 are defined as those models for which the TEST data has an overall fit of $R^{2}>0.91$ and $U_{T}<0.18$. A breakdown of the overall model fit is shown in Figure 57. On the left hand side of the figure is the $U_{T}$ for each individual state for both the ID data fit and the TEST data fit. It is a bar plot, with the threshold set at the defining value of 0.3 . Recall that for $U_{T}$, good models will have a value less than 0.3 , so good behavior in the left two plots of Figure 57 is bars pointing down, and the farther they point down, the better the model fits each individual state. In the ID data, the fit is poorest in surge and yaw rate. It is unusual then, that the TEST data fits surge very well, and the poorer fits are in roll and yaw. It is possible that this is due to a mismatch in trim values between the ID and

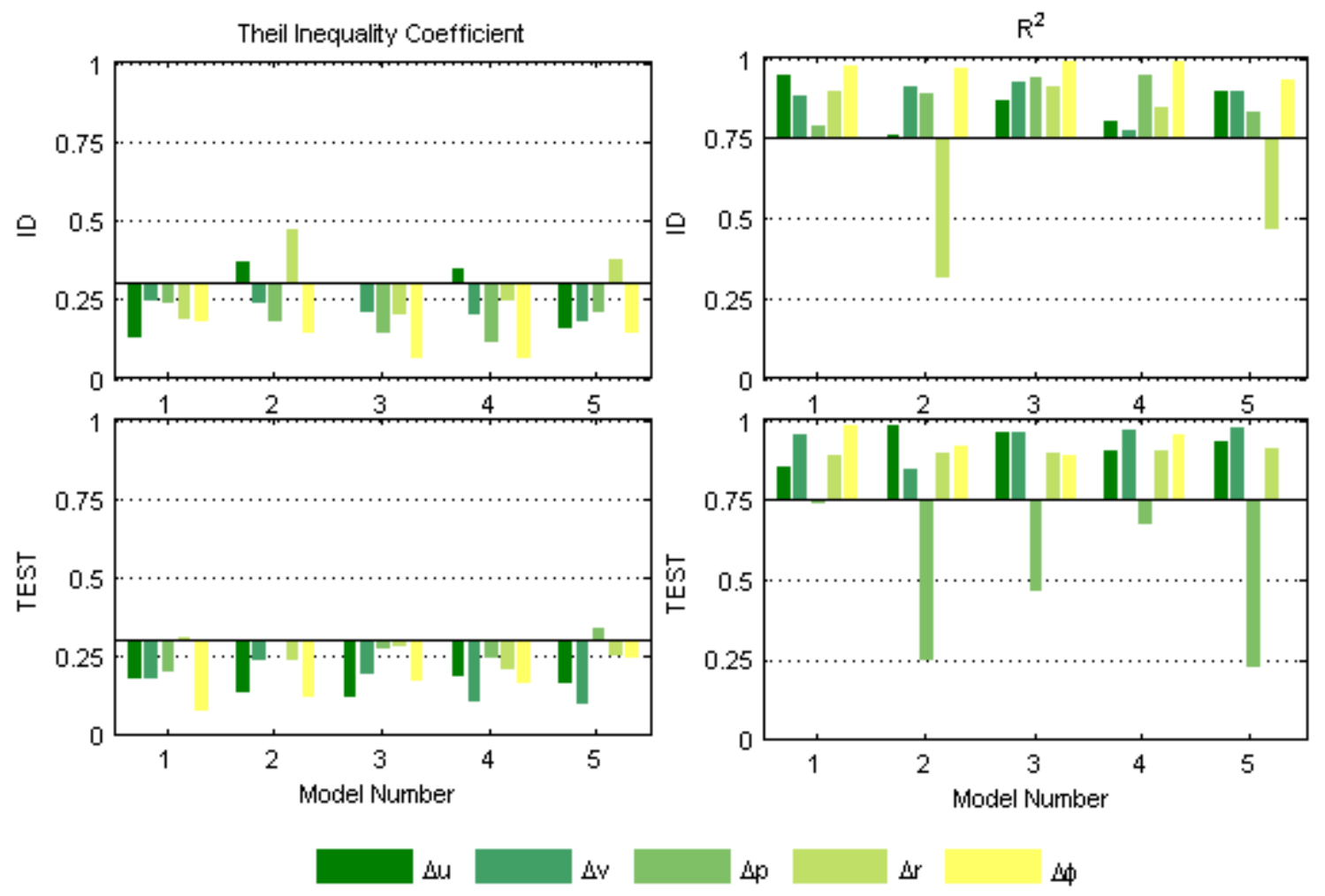

Figure 56: Breakdown of the best 5 Jib models by state showing difficulty matching the roll degree of freedom of the TEST data. 
TEST data, as when they were paired it was not a priority to have them match. This was done purposefully to see whether models from different trim conditions could adequately match independent TEST data in different conditions.

On the right hand side of the graph is a similar depiction for the $R^{2}$ fit of both the ID and TEST data. The threshold is set at 0.75 , so in these plots bars going up represent good fits. In the ID data, yaw is again showing a poor fit in Models 1 and 2, and in the TEST data, the poorest fit is in roll, with all five models showing an $R^{2}$ in roll of less than 0.75 . This supports the hypothesis from the previous section that roll is the largest driver of system dynamics for the sailing yacht.

To investigate the fit farther, we will turn to the breakdown of the Theil inequality coefficient described in Section 6.4. Recall that $U_{M}$ is a measure of the bias in the model, US the variance, and $U_{C}$ the covariance. Also recall that in a perfect model there would be no bias or variance, and the $U_{C}$ would be 1 . Figure 57 shows each of these proportions for each of the states for all five of the best Jib models. Ideally, $U_{C}$ should point up and be the tallest bar, and both $U_{M}$
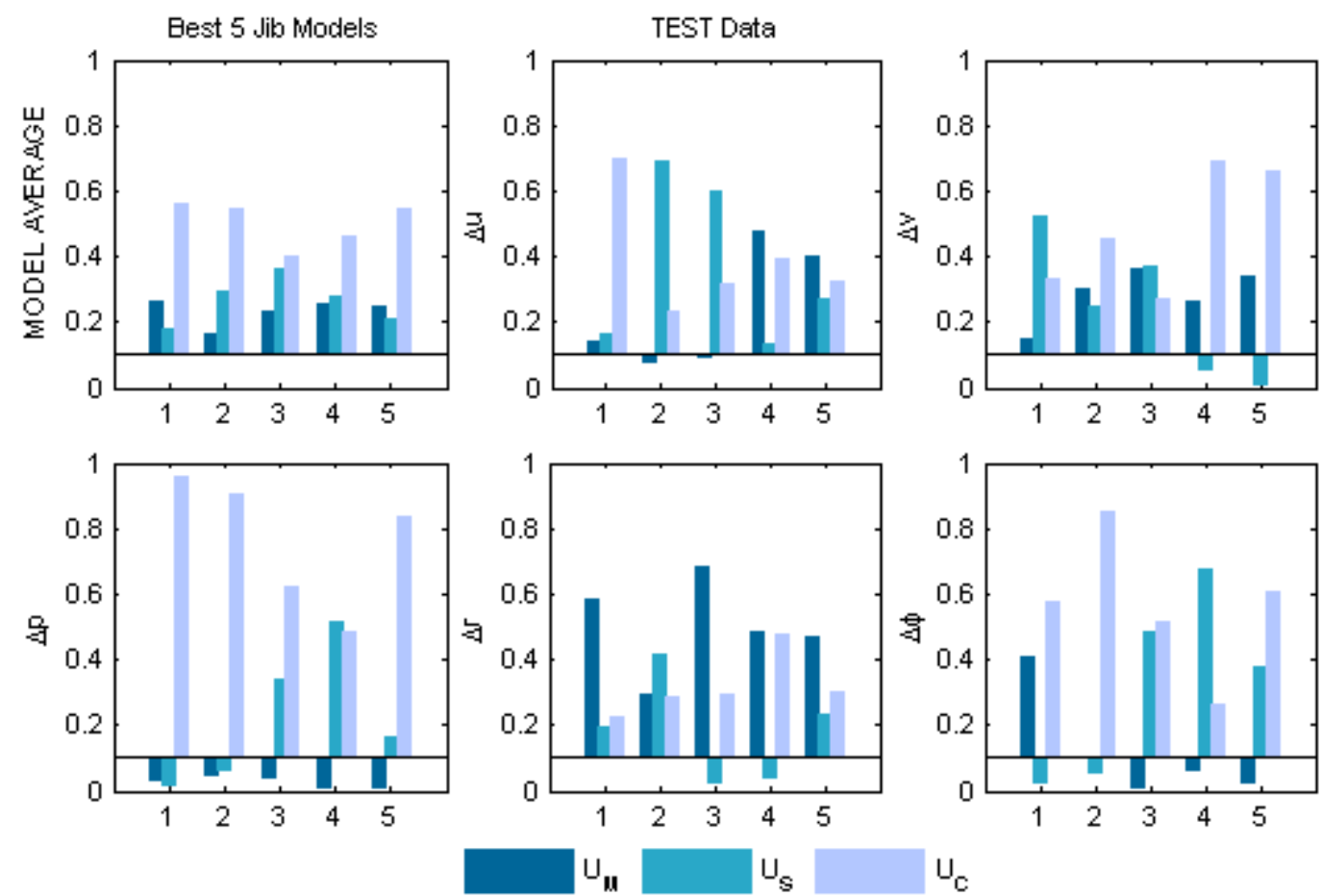

Figure 57: Theil error proportions for the best 5 Jib models reported for the entire model fit, as well as individually by state showing high bias proportions in yaw. 
and $U_{S}$ should point down (the threshold is set at .1 as discussed in Section 6.4). Overall, the data indicates that bias error, $U_{M}$, is very prevalent in the model's prediction of sway velocity and yaw rate. This bias is related to largely differing trimmed boat speeds between the ID and TEST data, as shown in Table 10. It should be noted that the model fit criteria is achieved in spite of these large differences.

Table 10: Difference in ID and TEST data trimmed surge velocities

\begin{tabular}{|c|c|c|c|}
\hline $\begin{array}{c}\text { Jib Model } \\
\text { Number }\end{array}$ & ID trimmed Sway $(\mathrm{m} / \mathrm{s})$ & $\begin{array}{c}\text { TEST trimmed Sway } \\
(\mathrm{m} / \mathrm{s})\end{array}$ & Difference \\
\hline 1 & 0.28 & 0.06 & $-78 \%$ \\
\hline 2 & 0.25 & -0.06 & $-124 \%$ \\
\hline 3 & 0.08 & 0.32 & $+250 \%$ \\
\hline 4 & -0.13 & 0.32 & $+346 \%$ \\
\hline 5 & 0.01 & 0.32 & $+310 \%$ \\
\hline
\end{tabular}

\subsubsection{Best Estimated Jib Model}

The best model fit to the TEST data achieved is $R^{2}$ of 0.94 and $U_{T}$ of 0.17 . The state space model for this best fit is shown in Eq. (8.15):

$$
\begin{aligned}
\vec{x}_{0} & =\left[\begin{array}{llllll}
2.37 \mathrm{~m} / \mathrm{s} & 0.25 \mathrm{~m} / \mathrm{s} & 0 & 0 & 0.22 \mathrm{rad}
\end{array}\right]^{T} \\
{\left[\begin{array}{c}
\Delta \dot{u} \\
\Delta \dot{v} \\
\Delta \dot{p} \\
\Delta \dot{r} \\
\Delta \dot{\phi}
\end{array}\right] } & =\left[\begin{array}{ccccc}
-0.021 & 0.119 & 0.086 & 0.169 & 0.1344 \\
-0.022 & -0.270 & 1.930 & -0.038 & 0.0145 \\
0.024 & 0.004 & 0.068 & 0.050 & -0.119 \\
0.007 & 0.066 & -0.400 & -0.009 & -0.078 \\
0.000 & 0.000 & 1.000 & -0.001 & 0.000
\end{array}\right]\left[\begin{array}{c}
\Delta u \\
\Delta v \\
\Delta p \\
\Delta r \\
\Delta \phi
\end{array}\right]+\left[\begin{array}{cc}
0.023 & -0.038 \\
-0.382 & 0.013 \\
0.024 & -0.009 \\
0.076 & -0.016 \\
0.000 & 0.000
\end{array}\right]\left[\begin{array}{c}
\Delta \delta_{r} \\
\Delta \delta_{b}
\end{array}\right]
\end{aligned}
$$

Note that the initial conditions represented Eq. (8.15) are in the upper, higher energy range, of all the models estimated with a total boat velocity of $2.4 \mathrm{~m} / \mathrm{s}$ and roll angle of 13 degrees. The data used to estimate this model is shown in Figure 35. The fit to the ID data is shown in Figure 58, and the fit to the TEST data is shown in Figure 59.

Finally, the first model estimated with the OSLS estimation of linear sailing yacht dynamic models, is not included in the above analysis. The biggest difference is that there were 6 
maneuvers in its' ID data set, rather than three, as is true with all of the models mentioned in this section. This first model did meet the fit criteria showing that it is possible to use more than three maneuver segments to estimate the models as long as the data is of the proper quality, and it is documented in Appendix C.
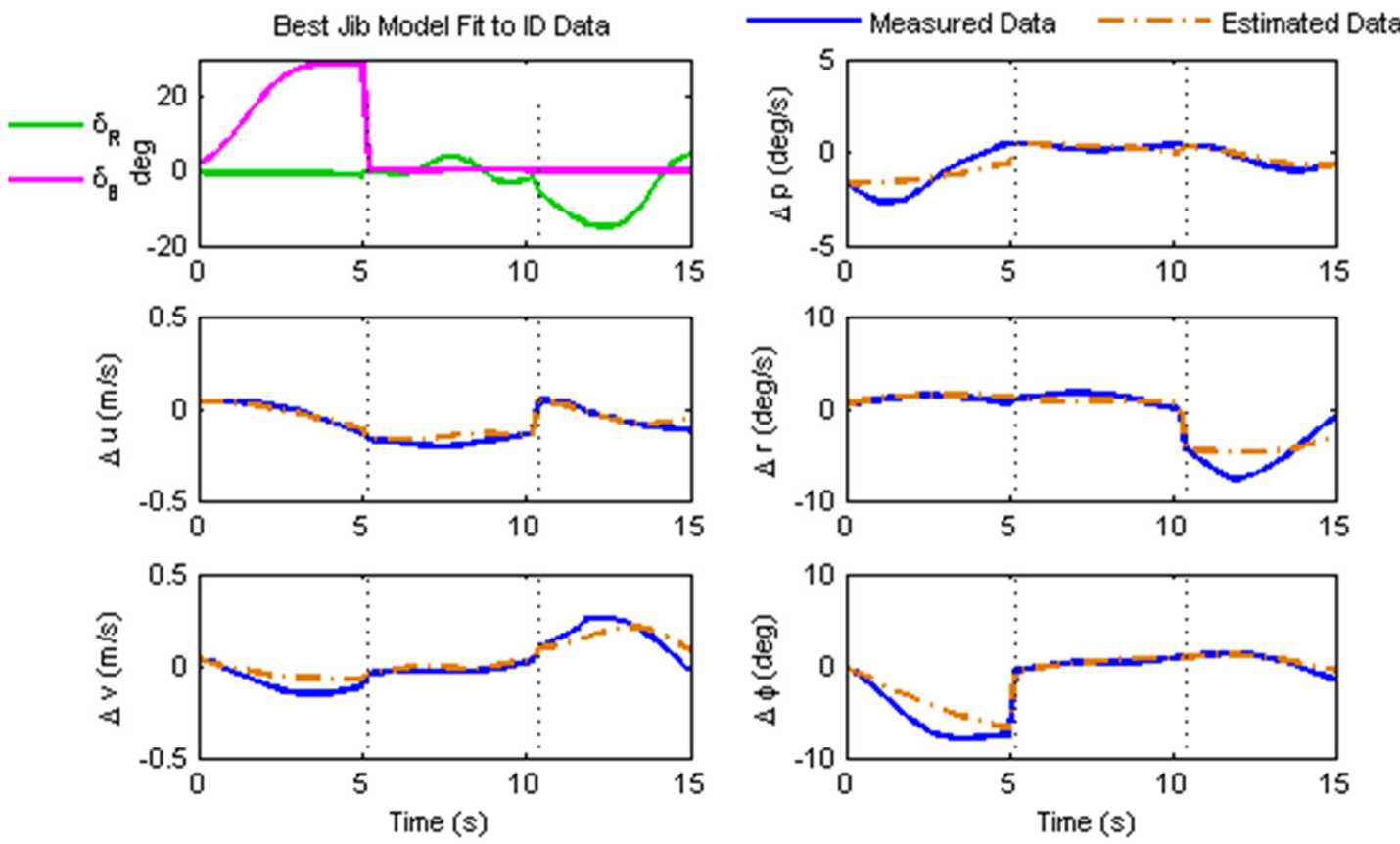

Figure 58: Best Jib Model Fit to the ID Data
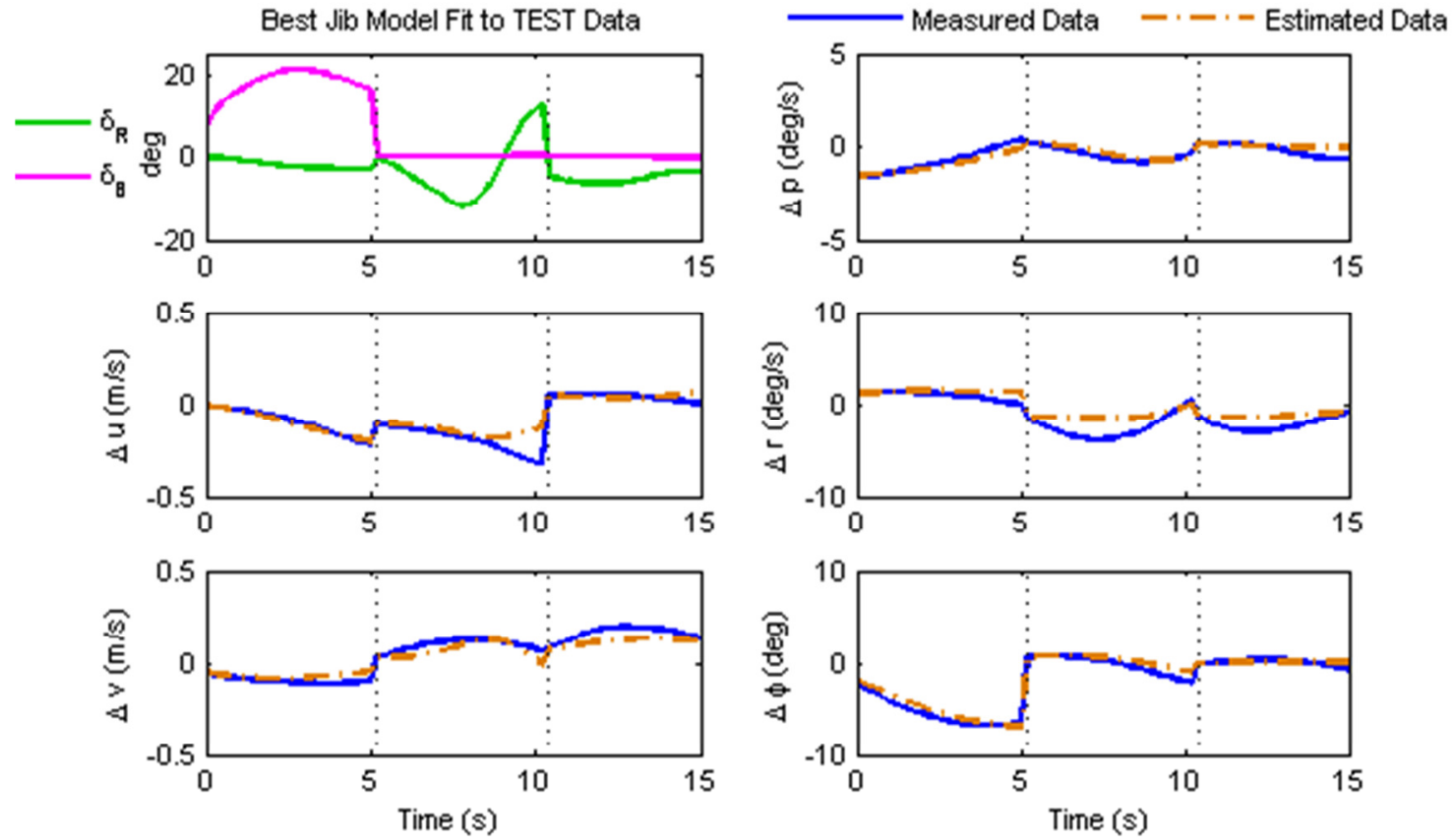

Figure 59: Best Jib model fit to the test data. 


\subsection{Estimated Genoa Models}

The Genoa configuration was very challenging when it came to selecting maneuvers for the data pre-processing. This configuration represents the largest sail area the boat can carry, and thus it corresponds to the slowest wind speeds. On some of the days during which data was collected in this configuration, the boat was operating at slow speeds and under five degrees of roll (recall the dynamic response data in Figure 24 and Figure 25). This fact decreased the amount of data used for the following analysis substantially because in order to accommodate the lack of dynamic roll response either a different estimation strategy or a different model would have to be used with this data. Overall this resulted in fewer models estimated than in the jib configuration. In total, 84 sets of ID and TEST data meet the $R^{2}$ and $U_{T}$ criteria. Of these 84,17 unique valid models were estimated for the Genoa configuration. 12 of these models are Type A models, and 5 are Type B models. With only 17 total models to work with, it is more difficult to perform the same analysis as with the $102 \mathrm{Jib}$ models. Also, as mentioned in Section 5.3.1, data in the upper wind range of this configuration is missing because of the decision to use the smaller sails if the wind was in the upper range. The following sub-sections are presented for completeness, and observations are made when appropriate which support some of the ideas observed in Section 8.4.

\subsubsection{Presentation of Modes for Genoa Models}

First to be analyzed are the 12 Type A models. Figure 60 is a plot of all the Modes $1 \mathrm{~A}$ from the Jib estimated models colored according to trimmed roll angle. Recall that in the Jib configuration it was clear the Mode 1A was highly influenced by roll angle (Figure 40). In the Genoa configuration, the lower limit on the roll angle range is $0.1 \mathrm{deg}$, whereas in the Jib configuration for Type A models it was 7 degrees. Therefore, the models for the Genoa configuration are showing trim points at low roll angles that were not present in the Jib models. The upper roll angle limits are very close, about 14 degrees, for which only one model exists in the Genoa data. In the Jib Type A models, the higher roll angles were mostly clustered to the left of the imaginary axis, indicating stability, which is not the case with the one Genoa model. Since there is only one at this high range, no real conclusion may be drawn about it, and it would be advantageous to collect more data in the Genoa configuration at higher roll angles and wind speeds to investigate whether a similar trend is seen as that in the Jib data. Figure 60 shows most 
of the roots are clustered closer to the right hand side of the imaginary axis, likely because the system is being given less energy from the wind.

Figure 61 shows Mode 1A colored according to apparent wind speed, and it is very clear looking at Figure 60 and Figure 61 together that the lower wind speeds correspond to the lower roll angles, as is expected. Second order Mode 2A is showing more stability at low roll angles, which is the opposite of Mode 1A. Recall from the Jib models that Mode 2A (Figure 42) was the most difficult to draw conclusions about, and without more data no definite conclusions may be drawn about Figure 62 and Figure 63.

The Genoa data shows the higher roll angles are associated with points of sail closer to the wind, in the cases shown here that means less negative apparent wind directions. Even with the large quantity of data in the Jib configuration, this trend did not surface. A possible explanation is that on the lower wind days, it is more important for the sails to be well trimmed in order to get the best performance out of the boat. While this is true also of the Jib configuration, it is less sensitive as it is possible to have poor sail trim but still maintain boat velocity. This phenomena can very readily be seen in larger, heavier cruising yachts with more inertia than the test boat which will almost always move if the wind is up if the sails are moderately close to trimmed well. Of course, this is done by sacrificing performance.

Last for the Type A Genoa models is Mode 3A, which recall from the Jib data was widely spread along the real axis(Figure 44) and very dependent upon boat speed, with the slower speeds spreading farther into the left hand side of the complex plane (more stability). The Genoa data as shown in Figure 66 is very clustered around the origin for a similar boat speed range as seen in the Jib data. Without more data points it isn't possible to make real comparisons of this mode to the Jib data.

There are only 5 Genoa Type B models, which really isn't enough data points to make comparisons with the Jib Type B models. The first order Modes 1B, 2B, and 3B are shown respectively in Figure 67, Figure 68, and Figure 69. The second order Mode 4B in Figure 70, which corresponds to the highly roll dependent second order modes 1A of the Type A models, does support the idea that this mode is roll dependent, even though only 5 data points are available. 


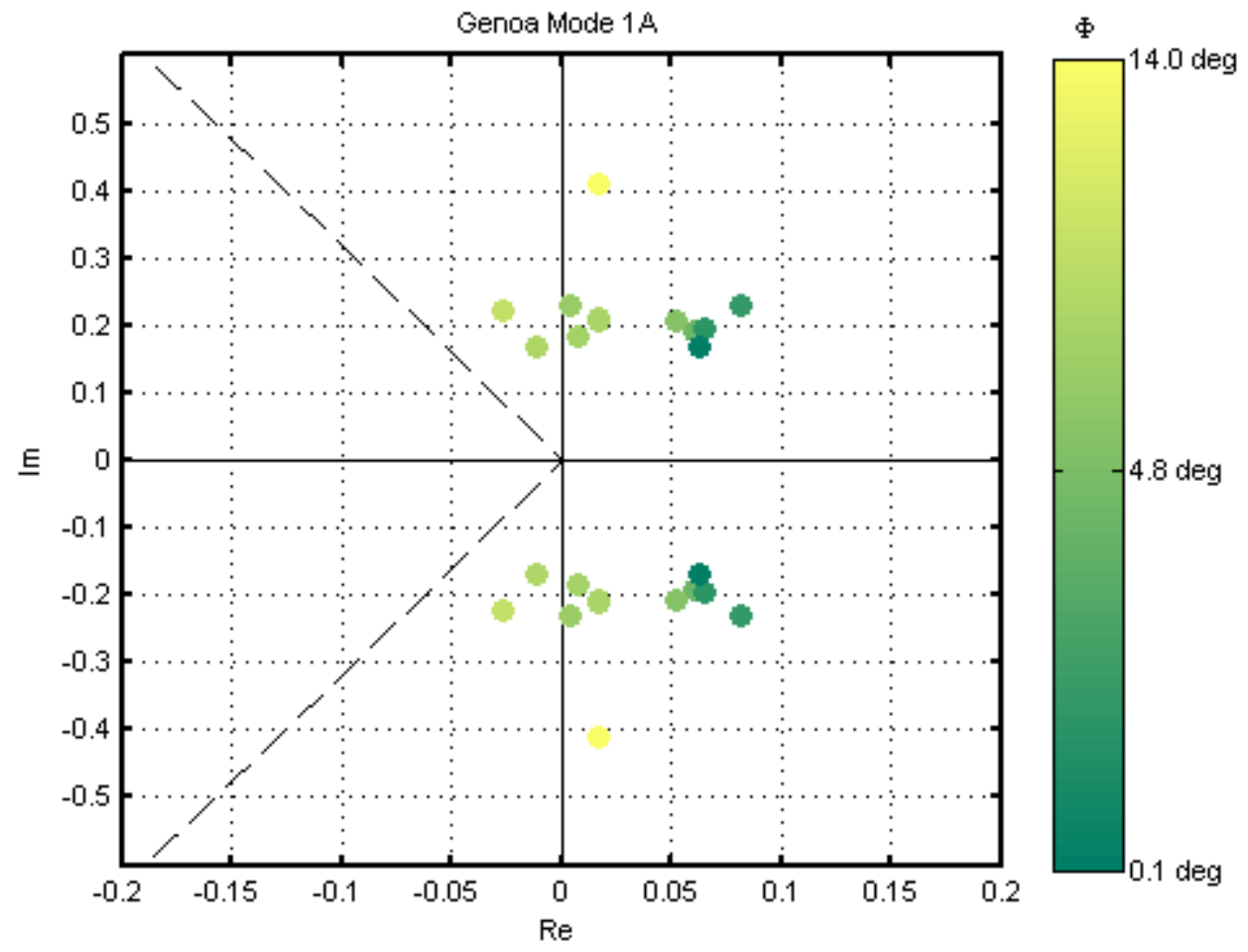

Figure 60: Genoa Modes 1A showing a trend with roll angle as there was with Jib Mode 1A.

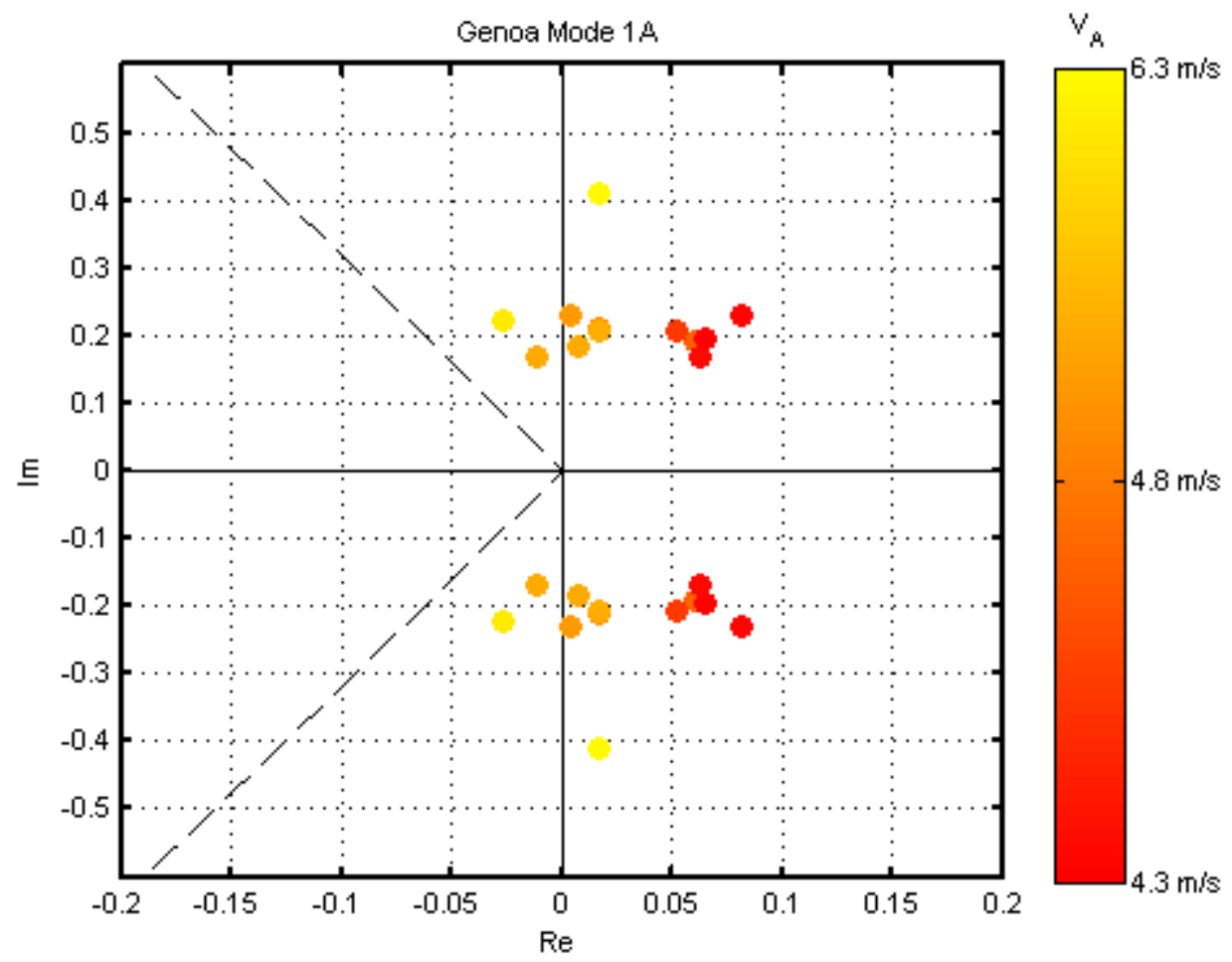

Figure 61: Genoa Modes 1A showing a trend with apparent wind speed. 


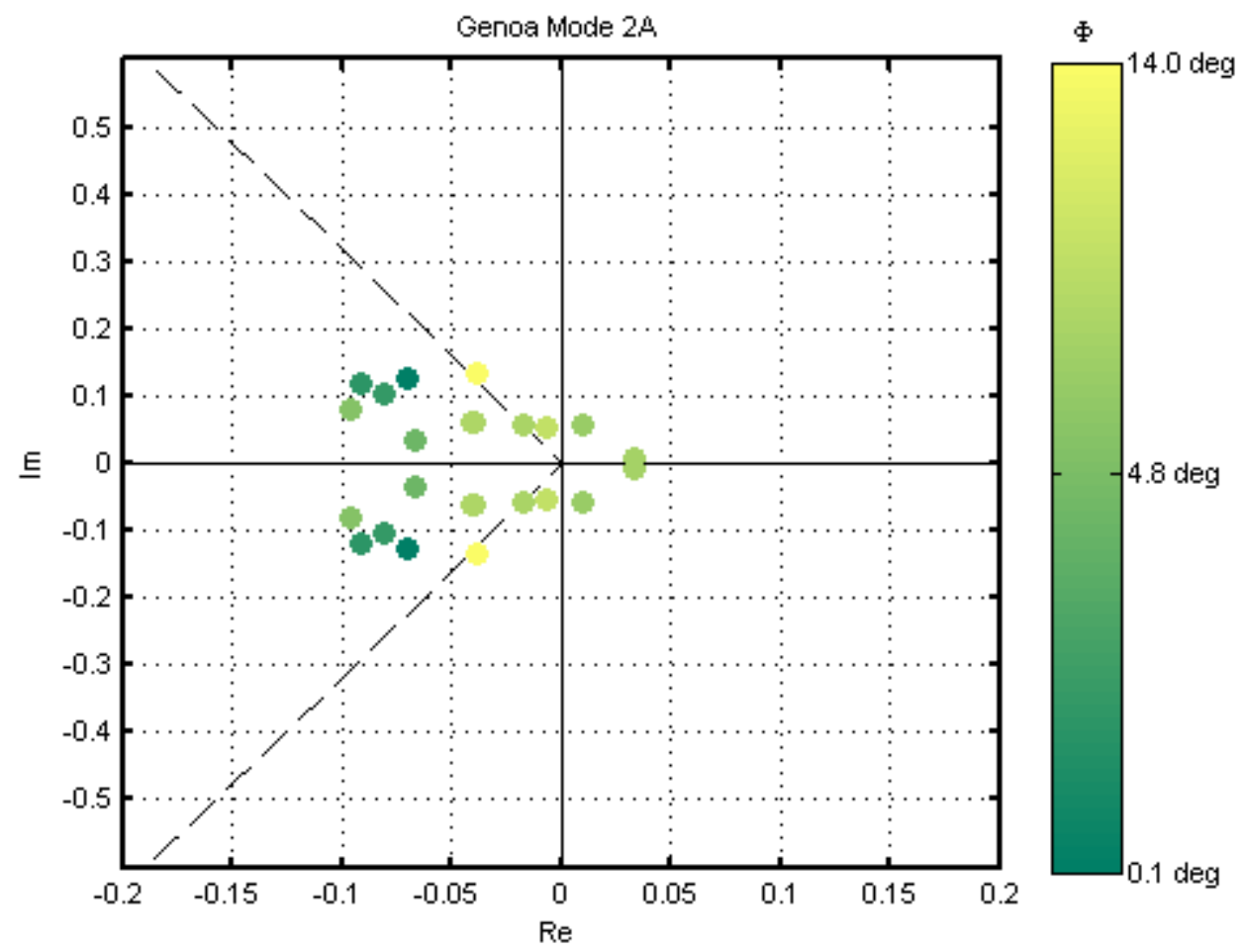

Figure 62: Genoa Mode 2A showing the lower roll angles clustered farther to the left of the complex plane and having damping less than 0.3

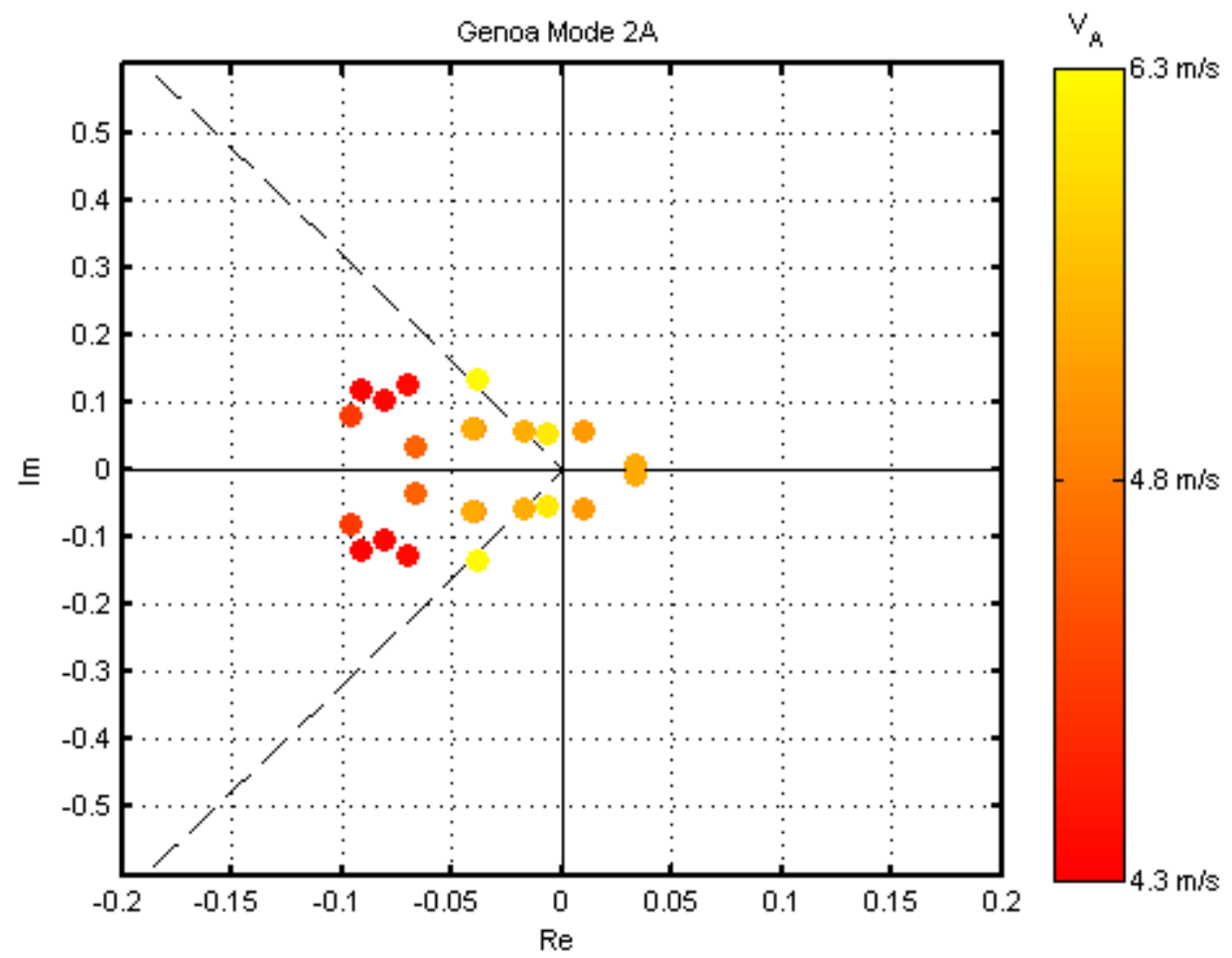

Figure 63: Genoa Mode 2A showing a the higher wind speeds correspond very well to the higher roll angles of Figure 62. 


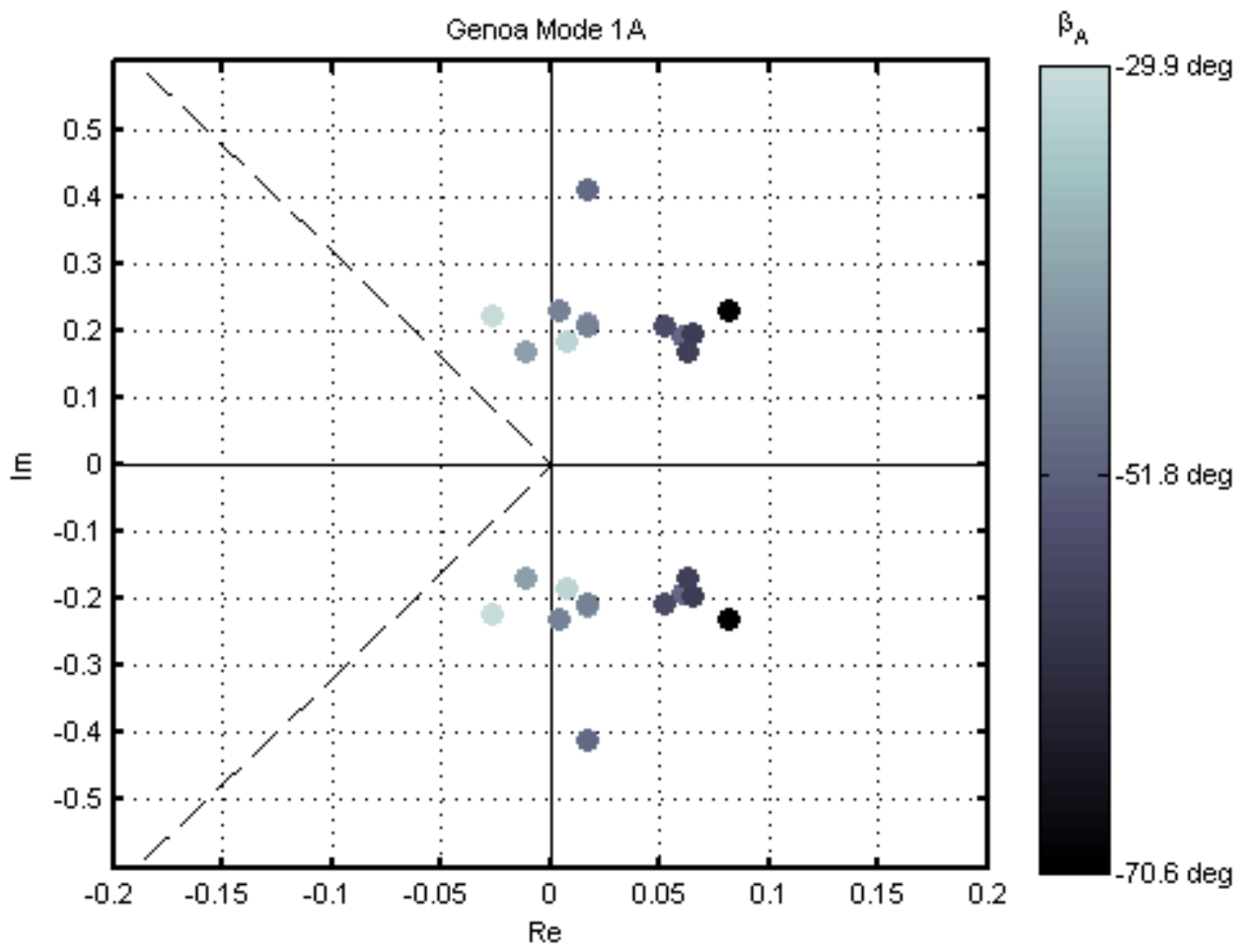

Figure 64: Genoa Mode 1A colored according to apparent wind direction.

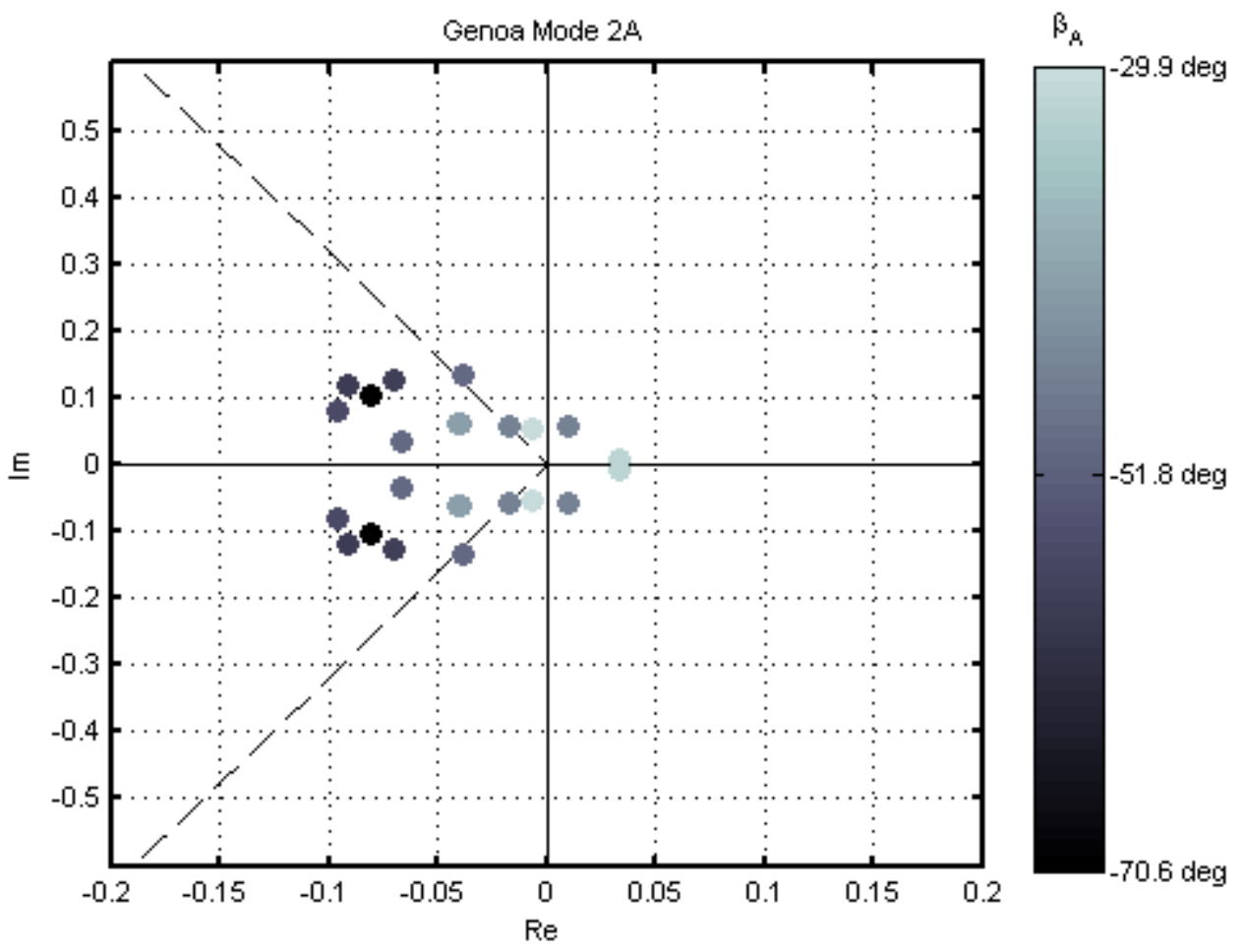

Figure 65: Genoa Mode 2A colored according to apparent wind direction. 

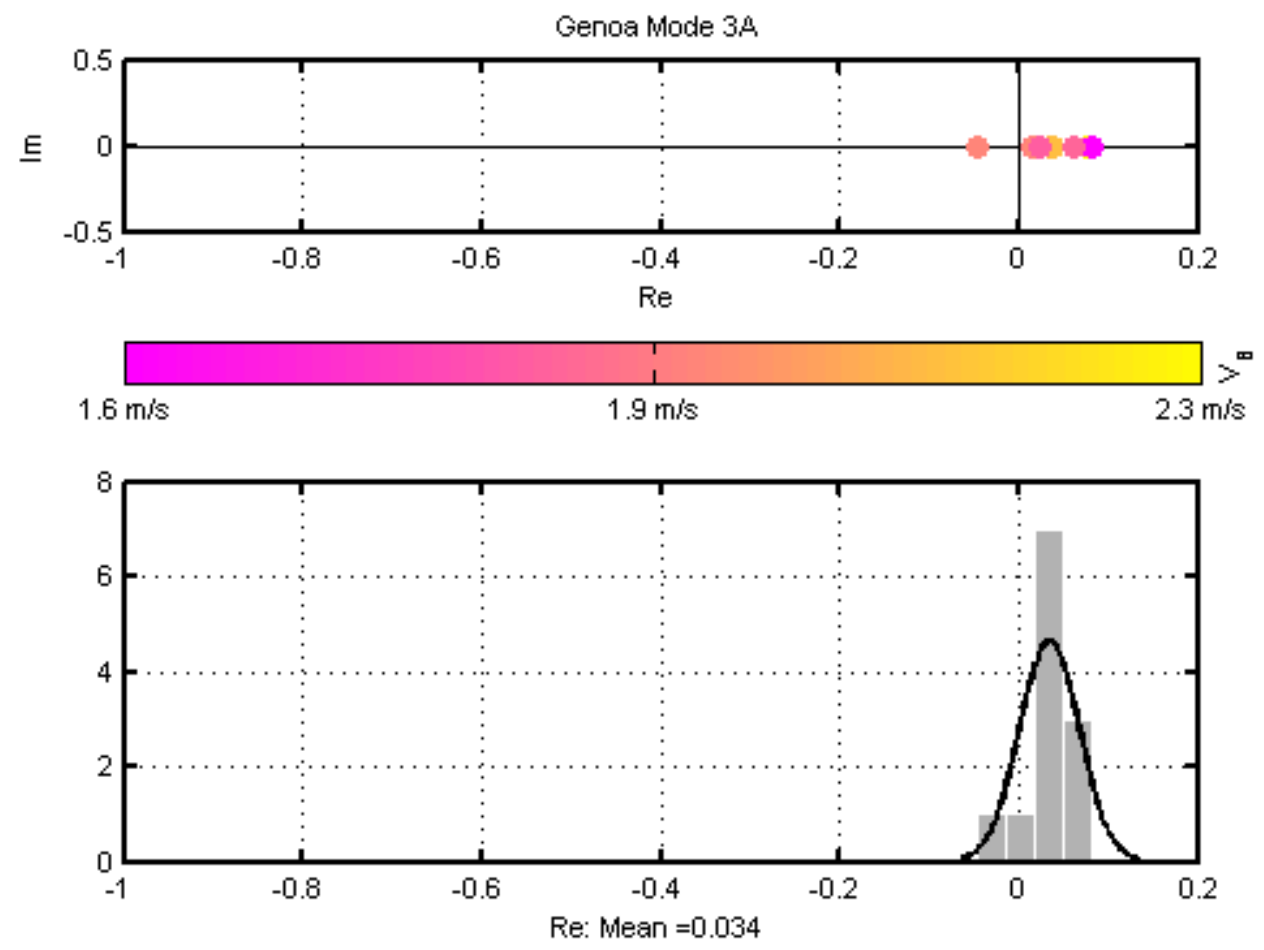

Figure 66: Modes 3A clustered to the right of the imaginary axis and centered at 0.034 , indicating instability.
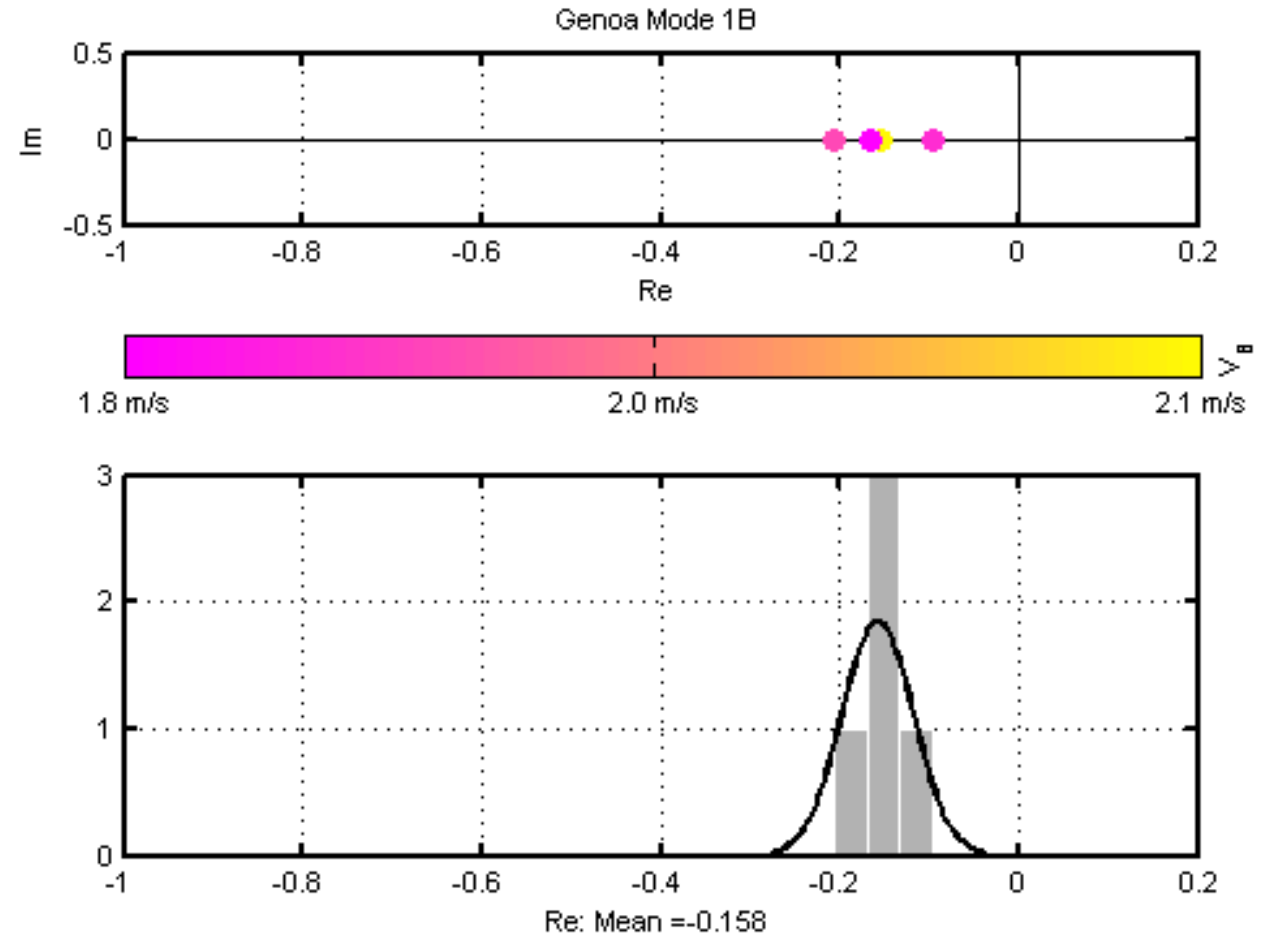

Figure 67: Genoa Mode 1B centered at $\mathbf{- 0 . 1 5 8}$, indicating stability. 

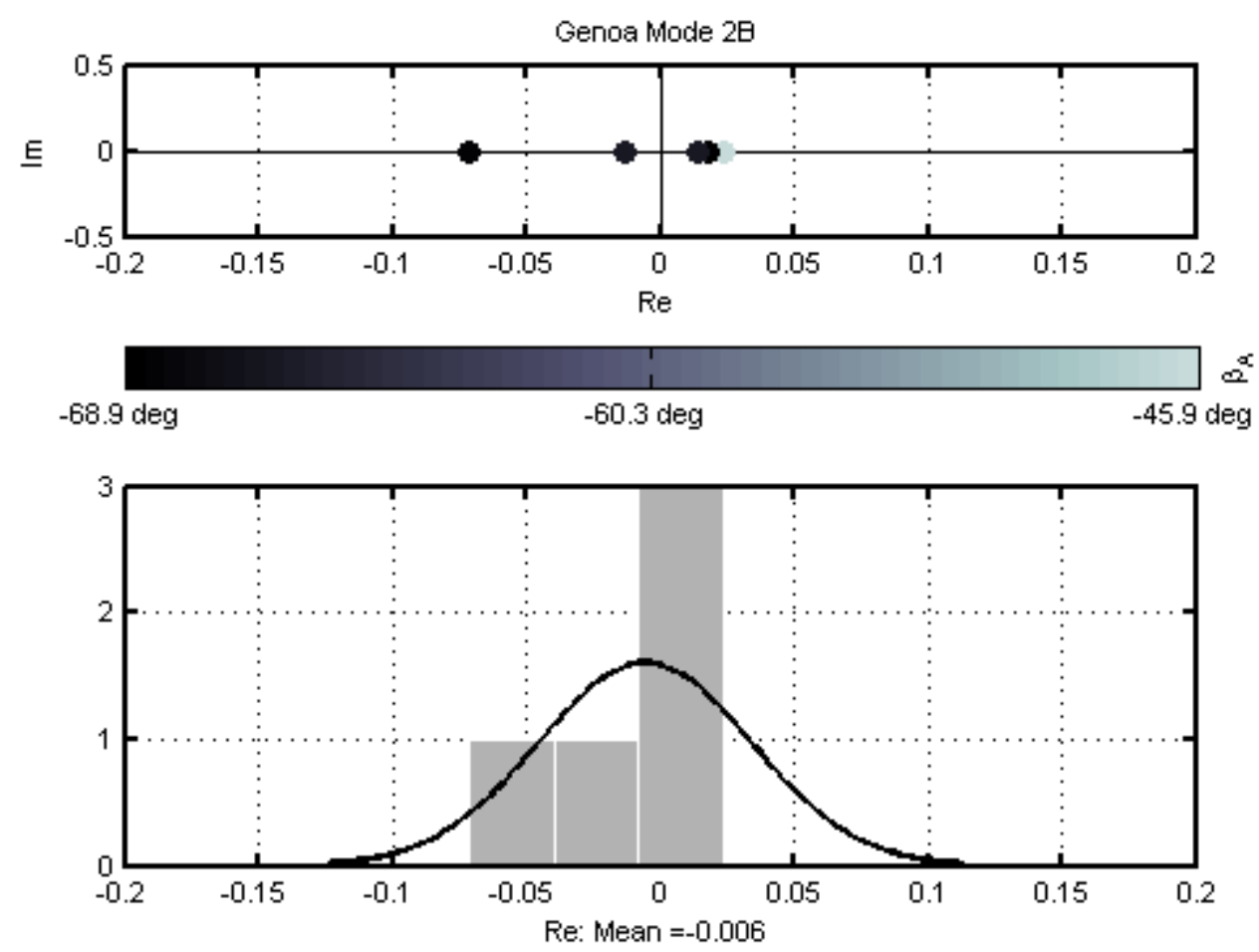

Figure 68: Genoa Mode 2B centered very close to the origin at $\mathbf{- 0 . 0 0 6}$ possibly indicating neutral stability of this mode.
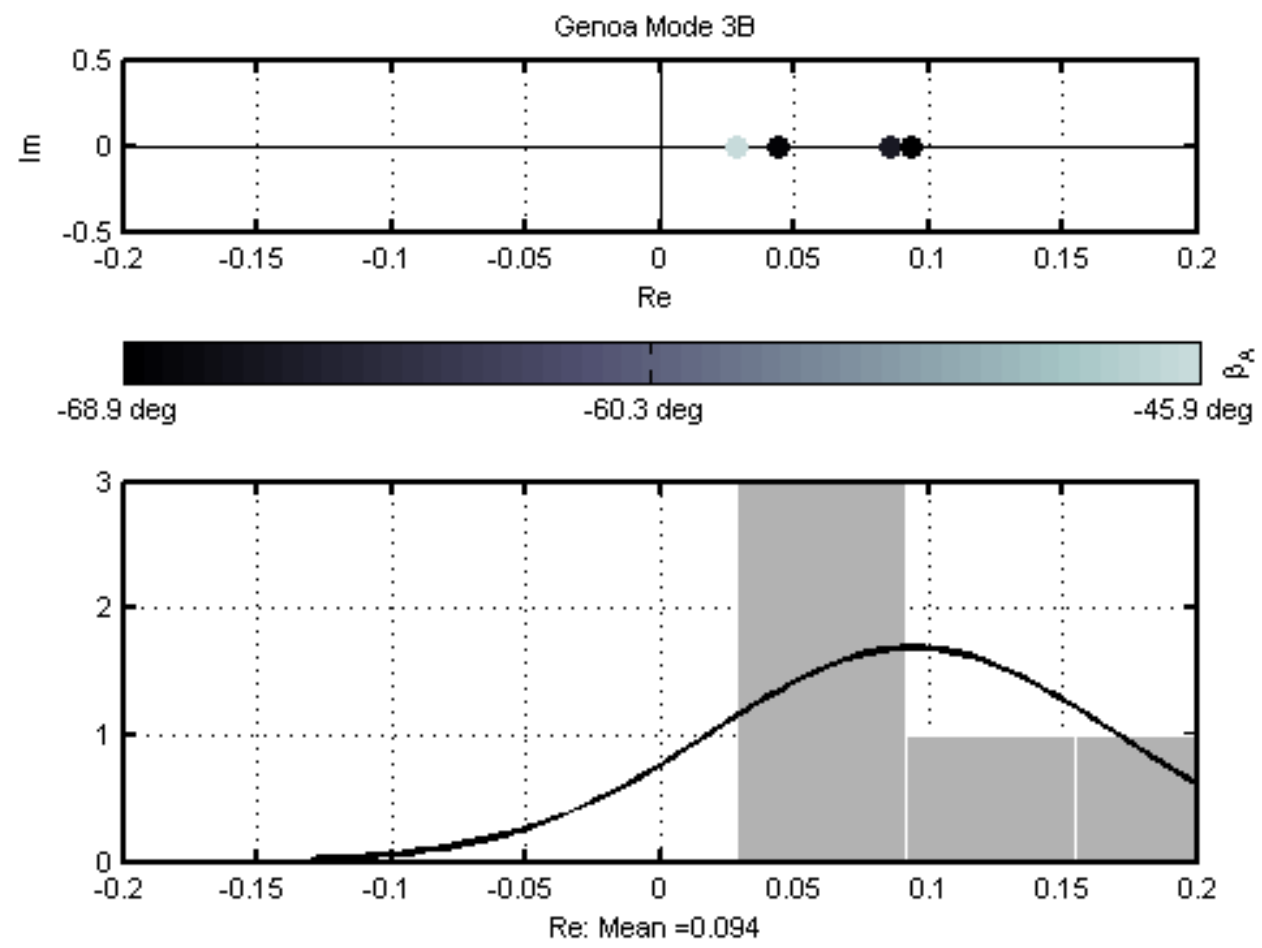

Figure 69: Genoa Mode 3B centered to the right of the imaginary axis at 0.094 indicating instability. 


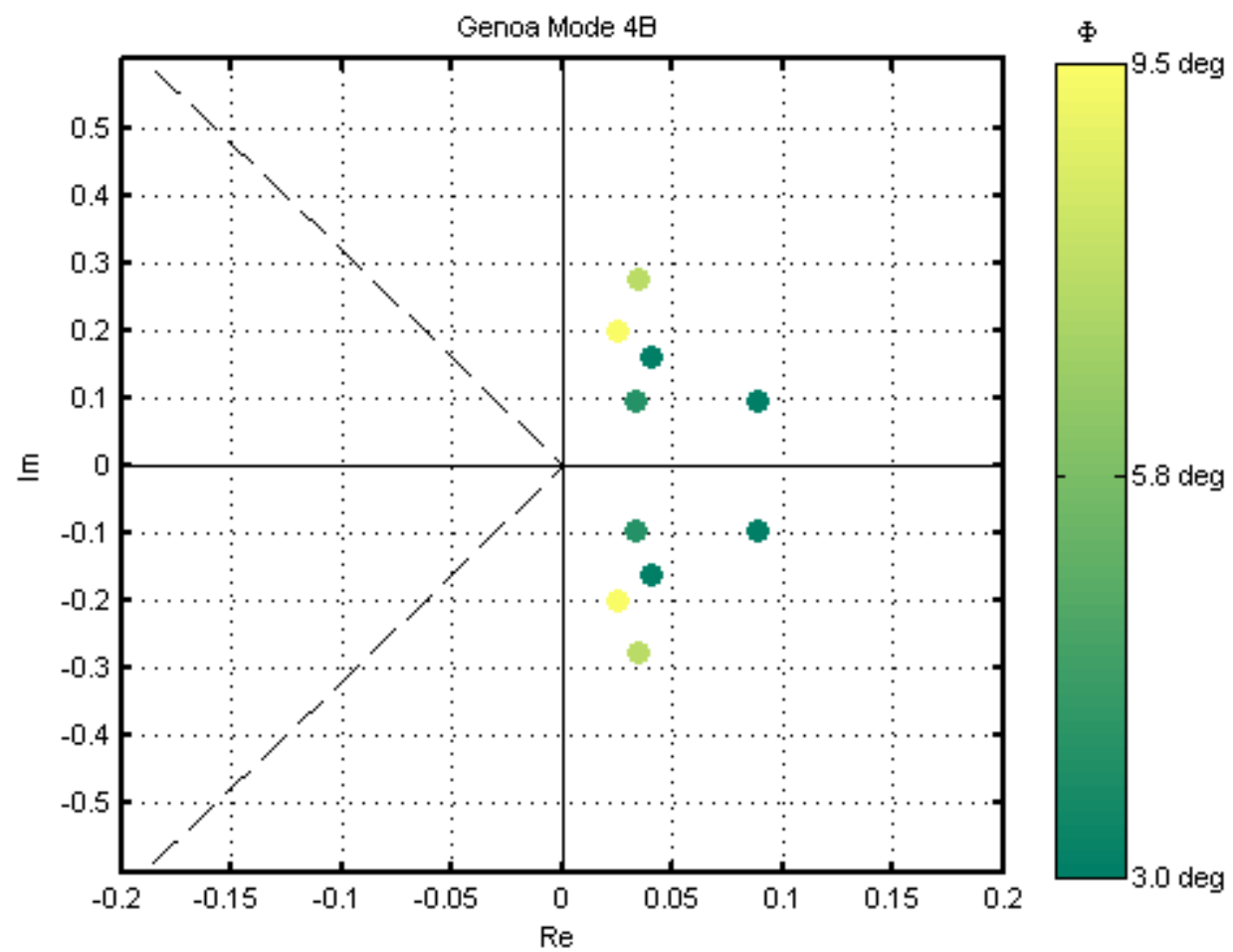

Figure 70: Genoa Mode 4B according to roll angle.

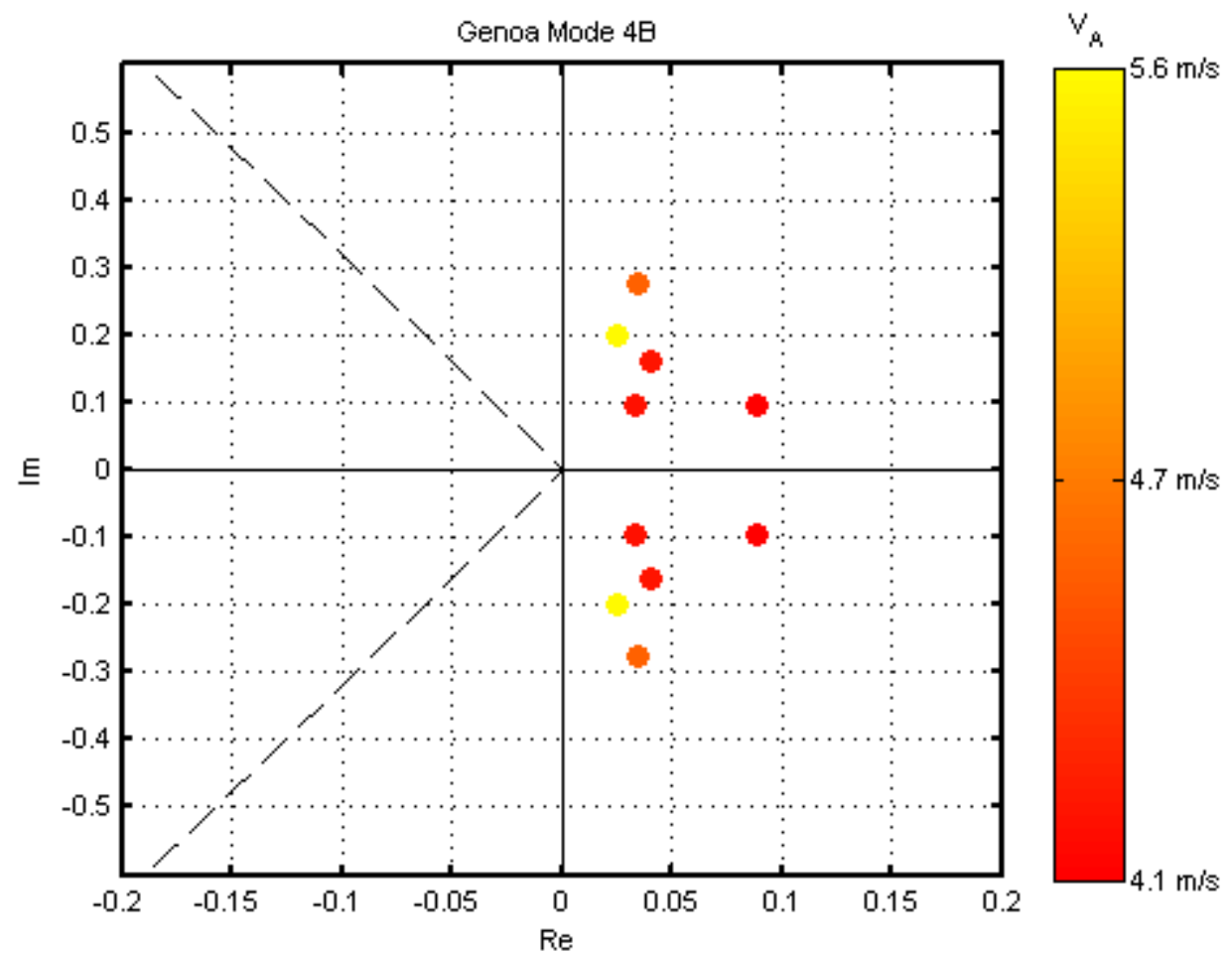

Figure 71: Genoa Mode 4B according to apparent wind speed. 
Table 11: Comparison of the ranges represented by the Type A and Type B models in the Genoa configuration.

\begin{tabular}{|c|c|c|c|}
\hline Model Type & Type A & Type B & $\begin{array}{c}\% \text { Difference in } \\
\text { Max }\end{array}$ \\
\hline Range for $V_{B}$ & $1.6-2.3 \mathrm{~m} / \mathrm{s}$ & $1.8-2.1 \mathrm{~m} / \mathrm{s}$ & $9 \%$ \\
\hline Range for $\phi$ & $0.1-14 \mathrm{deg}$ & $3-9.5 \mathrm{deg}$ & $32 \%$ \\
\hline Range for $V_{A}$ & $4.3-6.3 \mathrm{~m} / \mathrm{s}$ & $4.1-5.6 \mathrm{~m} / \mathrm{s}$ & $11 \%$ \\
\hline Range for $\beta_{A}$ & $-(30-71)$ deg & $-(46-70) \operatorname{deg}$ & $1 \%$ \\
\hline
\end{tabular}

Table 12: Comparison of the mean boat velocity, roll angle, apparent wind speed and apparent wind direction for the estimated Type $A$ and Type $B$ models.

\begin{tabular}{|c|c|c|c|}
\hline Model Type & Type A & Type B & \% Difference \\
\hline Mean for $V_{B}$ & $2.0 \mathrm{~m} / \mathrm{s}$ & $1.9 \mathrm{~m} / \mathrm{s}$ & $5 \%$ \\
\hline Mean for $\phi$ & $7.4 \mathrm{deg}$ & $5.5 \mathrm{deg}$ & $26 \%$ \\
\hline Mean for $V_{A}$ & $5.2 \mathrm{~m} / \mathrm{s}$ & $4.6 \mathrm{~m} / \mathrm{s}$ & $12 \%$ \\
\hline Mean for $\beta_{A}$ & $-48.0 \mathrm{deg}$ & $-62.9 \mathrm{deg}$ & $24 \%$ \\
\hline
\end{tabular}

Table 11 and Table 12 show that the three significant differences between the Type $\mathrm{A}$ and Type B models in the Genoa configuration are roll angle, apparent wind speed, and apparent wind angle. The Type A models correspond to $26 \%$ higher roll angles and $12 \%$ higher wind speeds than the Type B models, as shown in Table 12. This is similar to the trend shown in Table 9 for the Jib models. The Type A models represent points of sail $24 \%$ closer to the wind than the Type B models, which is part of the explanation for the higher roll angles as well. Figure 72 and Figure 73 show the distribution of boat velocity, roll angle, apparent wind speed and apparent wind direction within the ranges shown in the modal analysis. 

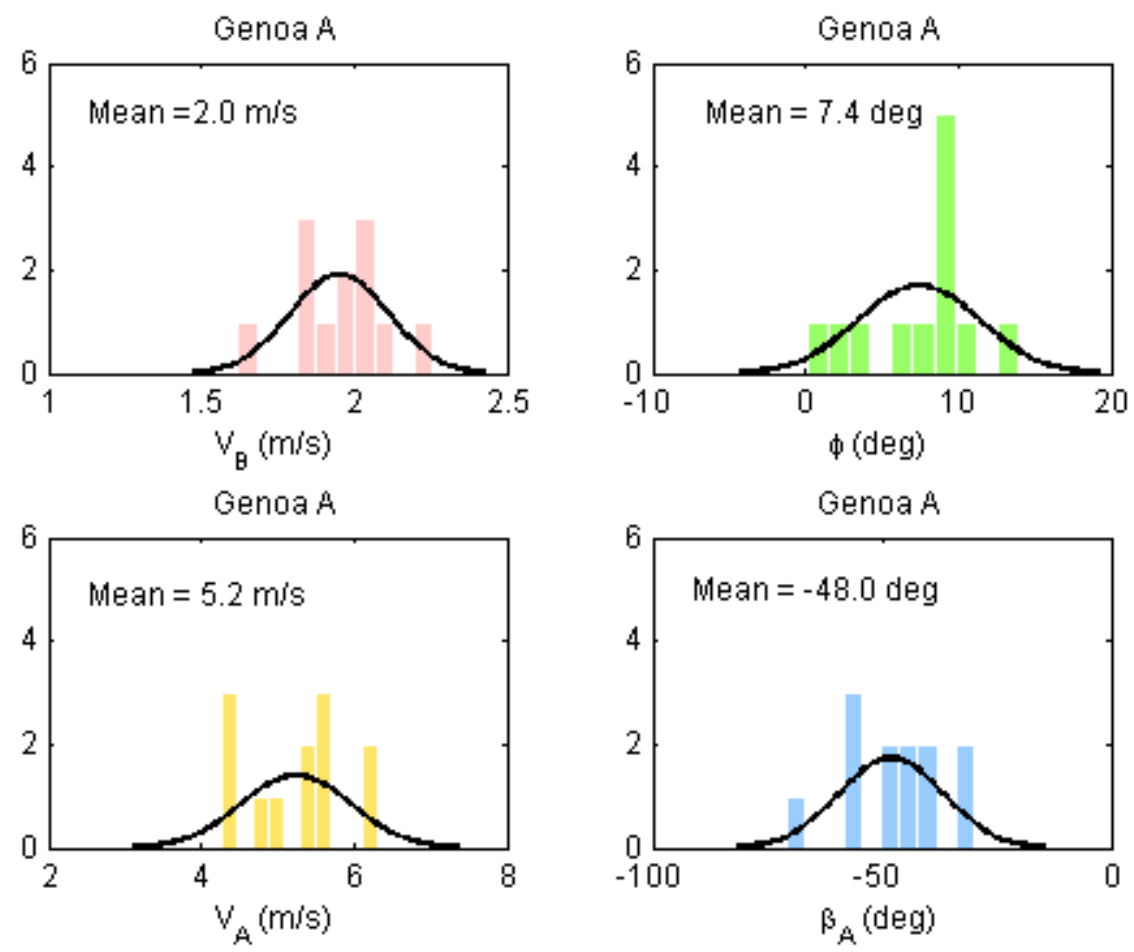

Figure 72: Distribution of trimmed boat velocity, roll angle, apparent wind speed and apparent wind angle for estimated Genoa Type A models.
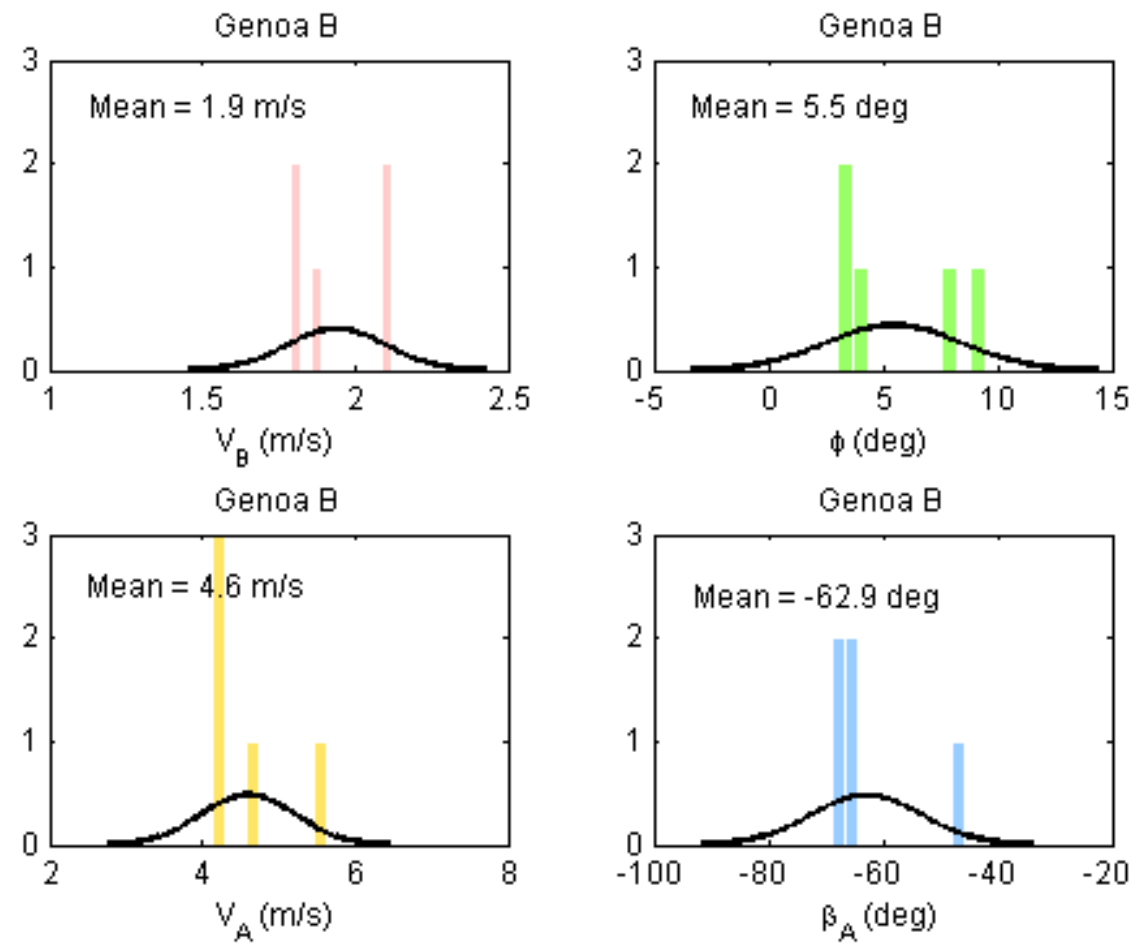

Figure 73: Distribution of trimmed boat velocity, roll angle, apparent wind speed and apparent wind angle for estimated Genoa Type B models. 


\subsubsection{Presentation of the Best Five Genoa Models}

The best five models in the category will again be presented. In the Genoa configuration the best five models have $R^{2}>0.84$ and $U_{T}<0.26$. The breakdown of the fit by states is show in Figure 74. According to both the $R^{2}$ and $U_{T}$ criteria, the models have difficulty matching yaw fairly consistently, especially in the TEST data. The breakdown of each state into the Theil proportions for the TEST data fit is shown in Figure 75. The bias proportion, $U_{M}$, is significantly high in both roll angle and roll rate which supports the conclusions from the modal analysis that the correct differentiator between models is the trimmed roll angle.
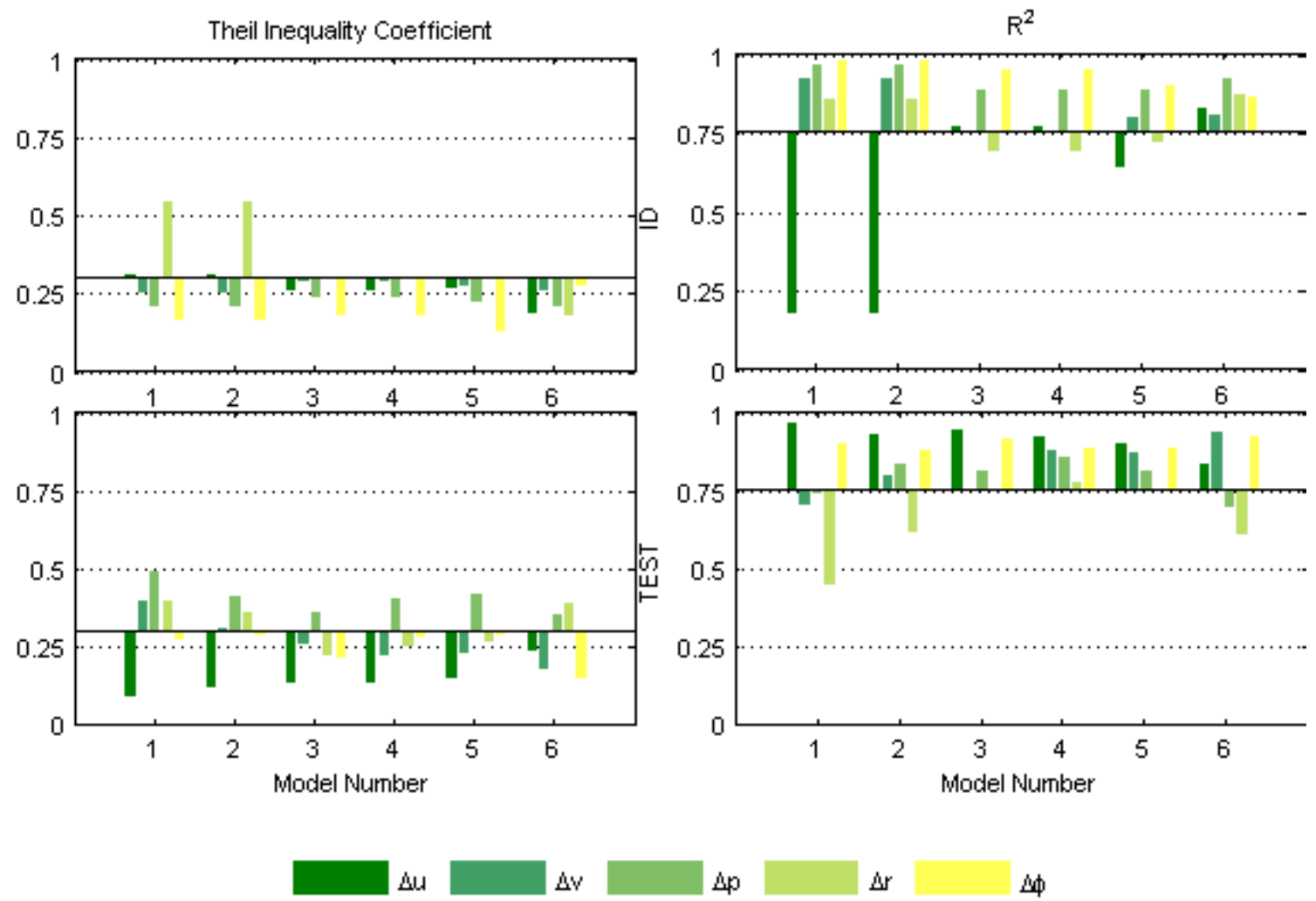

Figure 74: Breakdown of the best 5 Genoa models fit by state. 

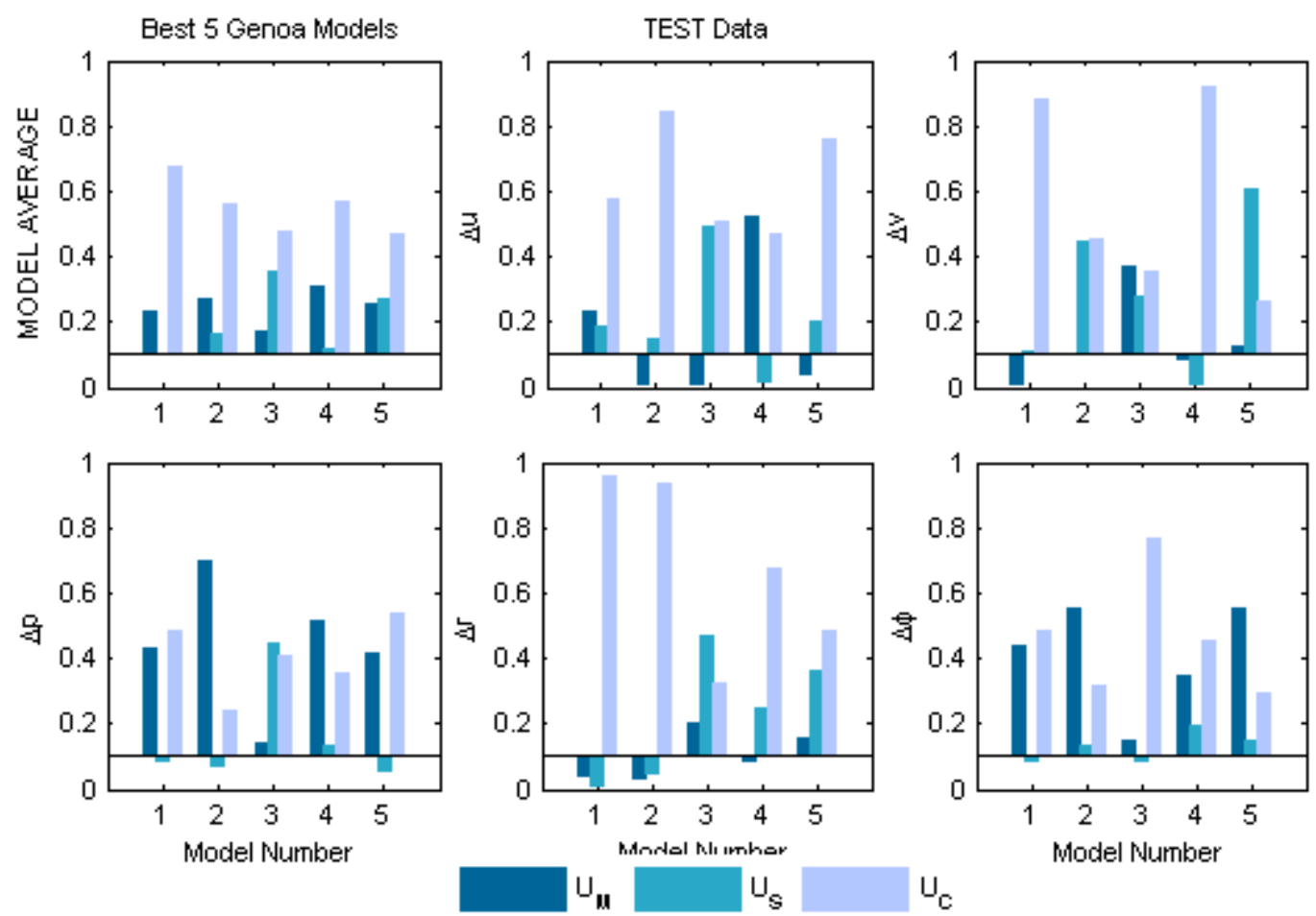

Figure 75: Theil error proportions for the best 5 Genoa models reported for the entire model fit, as well as individually by state.

\subsubsection{Best Estimated Genoa Model}

The best model fit to the TEST data achieved is $R^{2}$ of 0.88 and $U_{T}$ of 0.20 . The state space model is shown in Eq. (8.16):

$$
\begin{aligned}
& \vec{x}_{0}=\left[\begin{array}{llllll}
1.88 \mathrm{~m} / \mathrm{s} & -0.37 \mathrm{~m} / \mathrm{s} & 0 & 0 & 0.031 \mathrm{rad}
\end{array}\right]^{T} \\
& {\left[\begin{array}{c}
\Delta \dot{u} \\
\Delta \dot{v} \\
\Delta \dot{p} \\
\Delta \dot{r} \\
\Delta \dot{\phi}
\end{array}\right]=\left[\begin{array}{ccccc}
-0.006 & -0.071 & 0.653 & -0.135 & 0.372 \\
-0.098 & -0.300 & 1.823 & -0.261 & 0.300 \\
0.003 & 0.007 & 0.223 & 0.049 & -0.039 \\
0.058 & 0.196 & -0.716 & 0.083 & -0.235 \\
-0.001 & -0.001 & 0.955 & -0.010 & 0.008
\end{array}\right]\left[\begin{array}{c}
\Delta u \\
\Delta v \\
\Delta p \\
\Delta r \\
\Delta \phi
\end{array}\right]+\left[\begin{array}{cc}
0.064 & 0.012 \\
0.140 & 0.093 \\
0.005 & 0.035 \\
-0.092 & -0.041 \\
-0.001 & -0.007
\end{array}\right]\left[\begin{array}{l}
\Delta \delta_{R} \\
\Delta \delta_{B}
\end{array}\right]}
\end{aligned}
$$

Note the initial conditions of this model are in the lower energy range of the models presented in Section 8.5.1. The ID data used to estimate this model is shown in Figure 34. The fit to the ID data is shown in Figure 76 and the fit to the TEST data in Figure 77. 

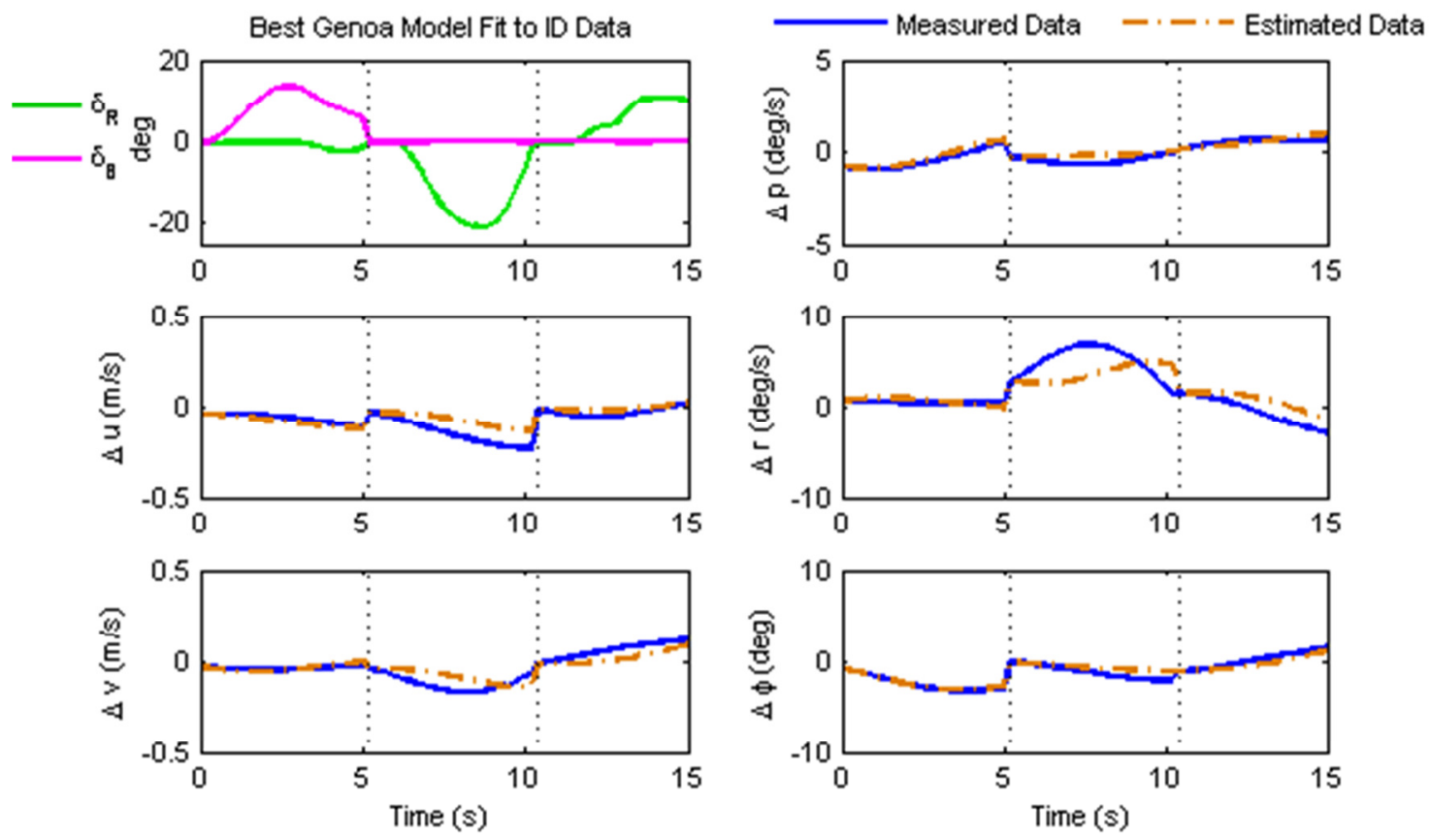

Figure 76: Best Genoa model fit to the ID data.
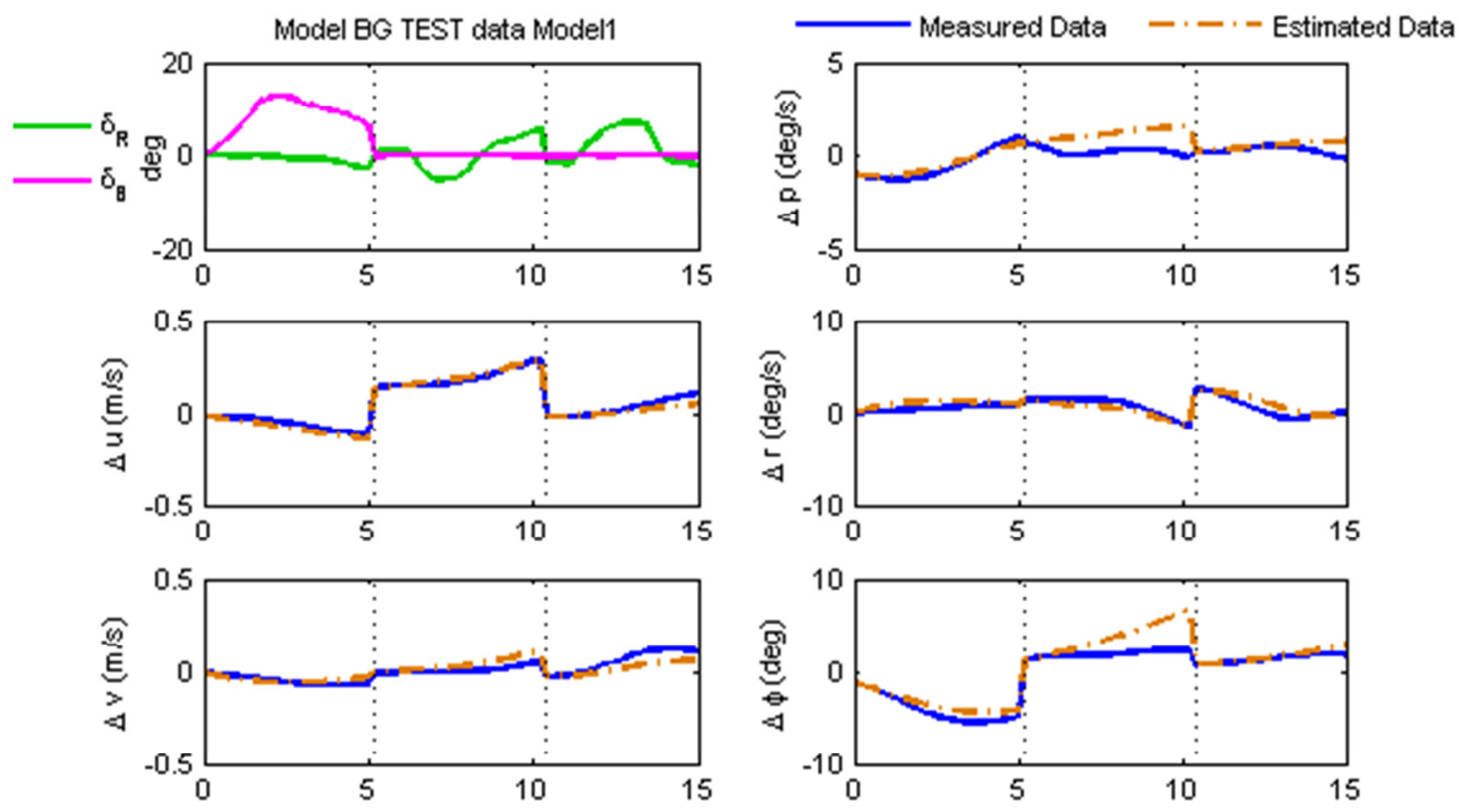

Figure 77: Best Genoa model fit to the TEST data. 


\subsection{Estimated Reef Model}

Only one model was estimated in the Reef configuration due to the lack of suitable data discussed in Section 5.3.3. The model fit to the TEST data is $R^{2}=0.87$ and $U_{T}=0.28$. The model is a Type A model, and is defined in Equation (8.17).

$$
\begin{aligned}
\vec{x}_{0} & =\left[\begin{array}{llllll}
2.51 \mathrm{~m} / s & -0.22 \mathrm{~m} / \mathrm{s} & 0 & 0 & -0.05 \mathrm{rad}
\end{array}\right]^{T} \\
{\left[\begin{array}{c}
\Delta \dot{u} \\
\Delta \dot{v} \\
\Delta \dot{p} \\
\Delta \dot{r} \\
\Delta \dot{\phi}
\end{array}\right] } & =\left[\begin{array}{ccccc}
-0.049 & 0.231 & -1.204 & 0.577 & -1.271 \\
-0.038 & -0.81 & 3.877 & -0.008 & -0.110 \\
-0.001 & -0.002 & 0.113 & 0.031 & -0.044 \\
0.008 & 0.059 & -1.429 & 0.030 & -0.018 \\
0.000 & 0.000 & 0.976 & -0.006 & 0.009
\end{array}\right]\left[\begin{array}{c}
\Delta u \\
\Delta v \\
\Delta p \\
\Delta r \\
\Delta \phi
\end{array}\right]+\left[\begin{array}{cc}
0.135 & -0.151 \\
0.161 & 0.075 \\
0.001 & -0.005 \\
-0.043 & -0.046 \\
-0.000 & 0.001
\end{array}\right]\left[\begin{array}{c}
\Delta \delta_{r} \\
\Delta \delta_{b}
\end{array}\right]
\end{aligned}
$$

The ID data set used to estimate Model BG is shown in Figure 78. Mode 1A is $0.03+/-0.28 \mathrm{i}$, Mode $2 \mathrm{~A}$ is $-0.03+/-0.09 \mathrm{i}$, and Modes $3 \mathrm{~A}$ is 0.020 . The model fit to the ID data in the first five seconds of simulation is shown in Figure 79. The model fit to the TEST data is shown in Figure 80. It is very encouraging that even with a very small sample of data one model was still estimated in this configuration, despite the fact that it represents the most dynamic, noisy, nonlinear conditions. As dealing with the upper wind range is important for an autonomous sailing yacht, it is recommended that this sailing regime be further characterized with tests in the future.
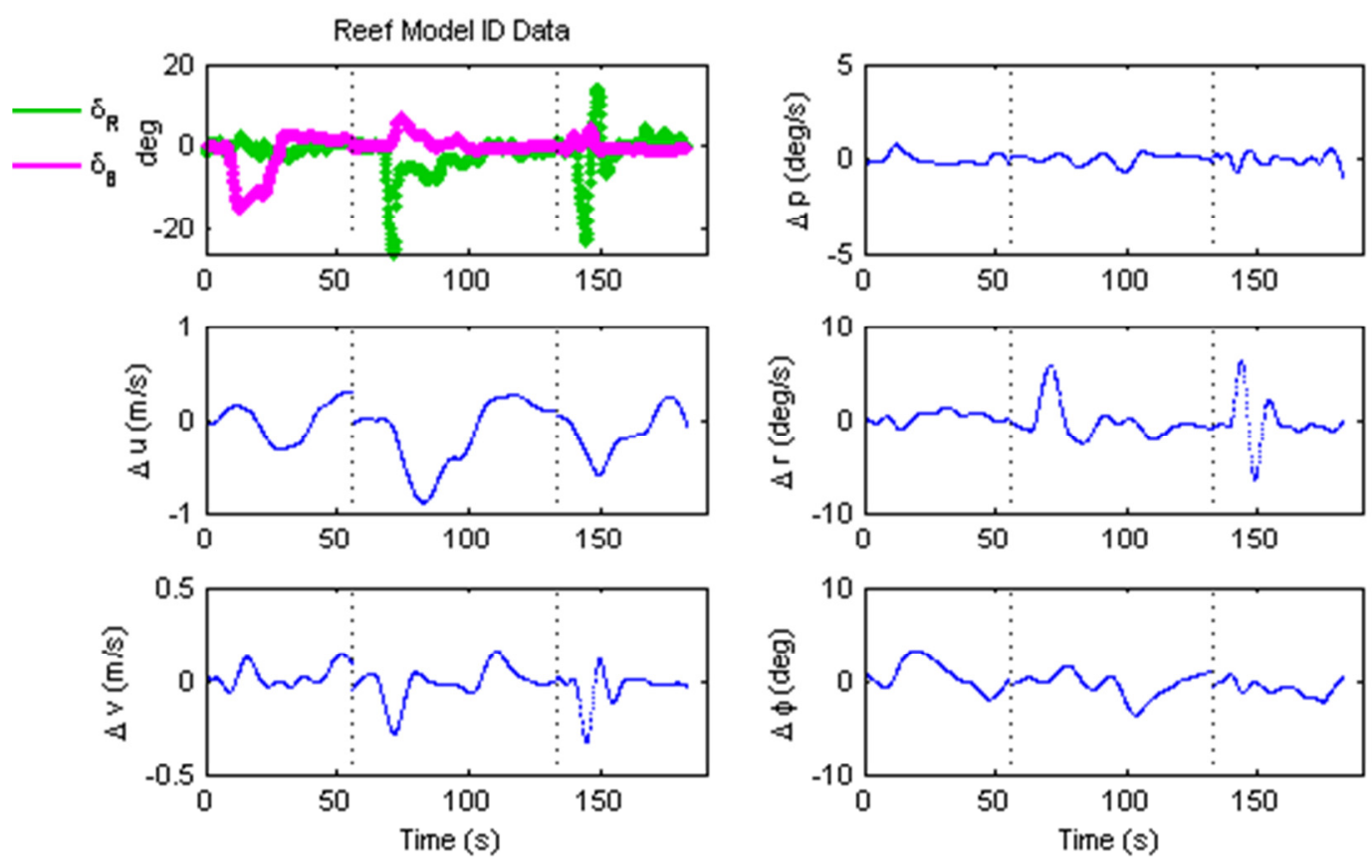

Figure 78: Data used to estimate the only Reef model. 

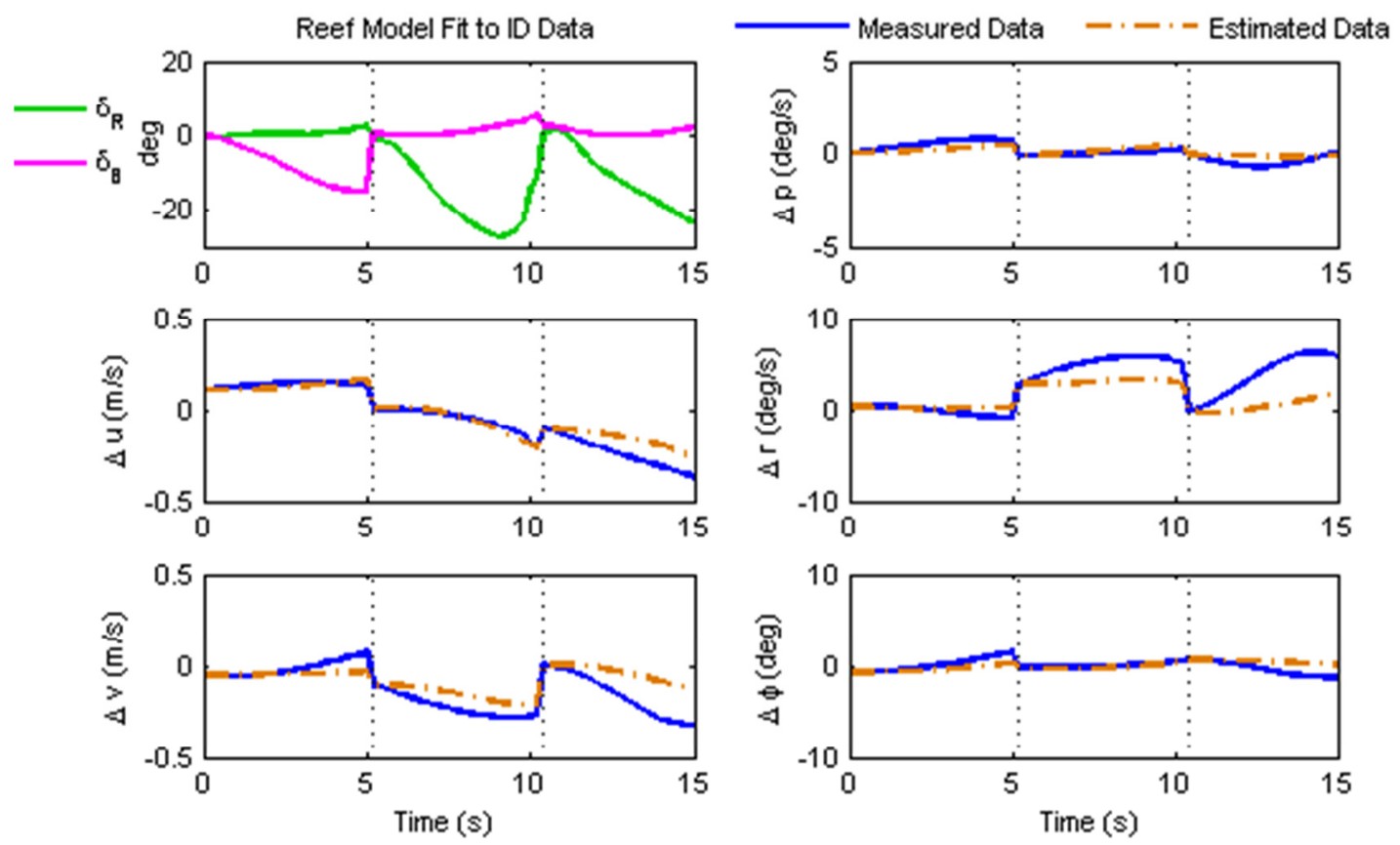

Figure 79: Reef fit to the ID data
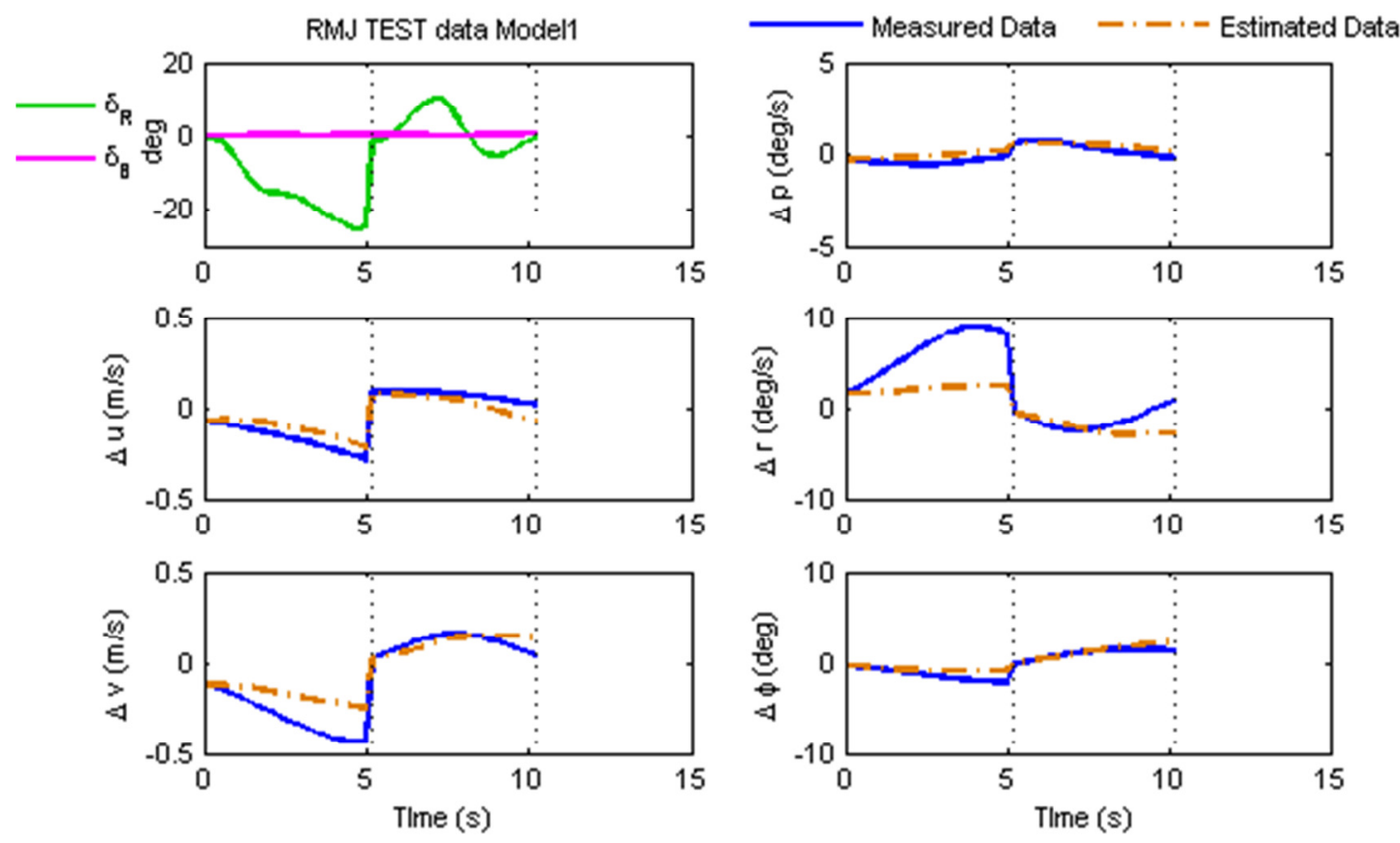

Figure 80: Reef fit to the TEST data. 


\section{Summary and Conclusions}

\subsection{Summary of Contributions to the Field}

Overall this dissertation has made significant advances in the understanding of sailing yacht dynamics through system identification of physics-based models from full scale sailing motion data. An expansive set of sailing data which includes all points of sail from low wind conditions through the maximum wind speed the boat can handle. This data is rich in control inputs and maneuvers which has made it suitable for a thorough investigation of full-scale sailing yacht dynamics in the full operational range of the boat. No set of data is available outside of proprietary sources at this time which covers both the expanse of weather conditions and control inputs, and as such even the reduced set of data included in this dissertation is of direct use to the sailing community for characterizing similar monohull yachts.

A modified physics-based non-linear model has been developed, and simulation of a monohull sailing yacht including physical sail deflection as a control input to the system has been tested in simulation and verified by full-scale data. This type of aerodynamic model is unique for a sailing yacht and it well suited to model linearization and control system design. The use of MLE formulation to identify the non-linear hydrodynamic derivatives of a sailing yacht from non-linear simulation data has been demonstrated as a viable method of non-linear hydrodynamic parameter estimation for sailing yachts.

The most significant contribution of this work is the linear system identification from fullscale data and corresponding modal analysis presented in Chapter 8. It has been shown that it is possible to estimate linear models in the three sail configurations: Full Main and Genoa (Genoa), Full Main and Jib (Jib), and Reefed Main and Jib (Reef). The detailed analysis of the Jib models has provided insight into how the sailing yacht behaves as a linear dynamic system. It has been concluded that roll angle is a prime differentiator among linear sailing yacht models and control system design should take that into account. Finally, the linear estimation and model evaluation process is readily repeatable on other monohull sailing yachts.

\subsection{Conclusions}

The over-arching goal of this dissertation was to provide insight into the modeling of sailing yacht dynamics using system identification techniques and full-scale data. Chapter 1 established 
that autonomous sailboats have advantages over existing platforms enabling them to perform missions ranging from the collection of ocean data to coastal surveillance. Chapter 2 established that there was a lack of information available about dynamic sailing yacht behavior that would be necessary for advanced control system design for an autonomous sailing yacht. It also surveyed the state of the art in modeling steady state sailing yacht dynamics, existing autonomous sailboat development, and system identification techniques that have been applied to ship estimation.

The full scale test yacht for the project was a $23 \mathrm{ft}$ day-sailer displacing about $2500 \mathrm{lbs}$. This yacht was advantageous for preliminary system identification because it is very representative of a wide range of monohull sailing yachts. It was outfitted with sensors to capture the motion data and inputs of the rudder and the sails, and a set of maneuvers, singlets and doublets, were designed specifically for linear system identification. In all over 20 hours of sailing motion data was collected for use in modeling the dynamics of sailing yachts.

\subsubsection{Non-linear Modeling and Estimation}

In order to begin applying system identification techniques to the sailing yacht, a physics based non-linear model was postulated in Chapter 3. The non-linear model includes both hydrodynamic and aerodynamic models. While the hydrodynamic models are simply taken from previous work, it was found that existing aerodynamic models for sailing yachts were not suitable for this study because they did not include a physical description of the way sails affect the dynamics of the system. Therefore, a new aerodynamic model describing the forces and moments on a sailing yacht was proposed and validated using full-scale data. The non-linear model was implemented in simulation, generating 'noise-free' data for use in exploring the application of system identification techniques to the sailing yacht system.

This non-linear simulation provided data for estimating non-linear hydrodynamic derivatives of a sailing yacht. The simulation data was created to mimic the full-scale tests that had been performed. The maximum likelihood estimation (MLE) method was used to estimate the nonlinear hydrodynamic derivatives of the simulated sailing yacht. The hydrodynamic derivatives for a yacht hull form are usually measured in the towing tank. Characterizing these derivatives from full scale motion data would be advantageous for both verifying towing tank data as well as avoiding the expense of the towing tank altogether. The resulting estimated derivatives produce a 
satisfactory time history, however, the numbers themselves are not aligned with the known derivatives. From a controls perspective, the estimation was satisfactory as the ability to reproduce the time history is what is important for control. From a boat performance perspective, it would be necessary to improve the estimation of the derivatives as the resulting numbers did not always match order of magnitude, and in some cases the sign of the known derivatives.

\subsubsection{Linear Modeling and Estimation}

The goal of this work was to increase understanding of sailing yacht dynamics for control system design, thus, characterization of the full set of non-linear parameters for the sailing yacht from full-scale motion data is not included in the study. Rather, the non-linear model was simplified to linear state space form, presented in Chapter 4. The primary assumption for linear systems is that the linear model is only valid near the trimmed operating point for which the model is defined. For a sailing yacht this means specifying a wind speed, wind direction, boat speed, and heel (roll) angle for which the model is defined. Preliminary studies which numerically linearized the non-linear model of Chapter 3 showed that wind speed and direction did not greatly affect vehicle response.

With the interest of analyzing and comparing several models at once, the one-shot least squares (OSLS) method was used to estimate the linear sailing yacht system dynamics from the full scale data. Computationally, OSLS is very fast and efficient, allowing rapid estimation of many models from the large quantity of data collected. In order for the OSLS method to accurately estimate models, the data it is presented with must be 'noise free' thus the full scale data is pre-processed before estimation. The data is segmented into individual maneuver segments averaging 40 seconds in length. Each of these maneuver segments is smoothed to remove any discontinuities and higher frequency oscillations in the measured signals. The smoothed maneuver segments are grouped into sets of ID data which include one rudder singlet, one rudder doublet, and one boom singlet.

Since prior linear models of a sailing yacht are unavailable for model validation purposes, estimated models are evaluated based on their ability to re-produce time histories of independent TEST data. Two criteria, $R^{2}>0.75$ and $U_{T}<0.3$, are specified at the end of Chapter 6 and estimated models must satisfy these criteria to be considered valid. The $U_{T}$ criteria is based on a well-known rule of thumb (Theil, 1958). The initial $R^{2}$ criterion is specified based on the 
Table 13: Summary of total valid linear models estimated from full-scale data.

\begin{tabular}{|c|c|c|c|}
\hline Model Configuration & Jib & Genoa & Reef \\
\hline $\begin{array}{l}\text { Number of successful } \\
\text { ID/TEST data pairs }\end{array}$ & 1211 & 84 & 1 \\
\hline $\begin{array}{c}\text { Number of Unique Models } \\
\text { Estimated }\end{array}$ & 102 & 17 & 1 \\
\hline
\end{tabular}

knowledge that there will be inherent uncertainty in the model structure, bias and variance in the measurements, and the limitations of the estimation algorithm utilized. The modal analysis also revealed the boat modes have long time constants, which gives a controller ample time to respond to changes and maintain system stability. Table 13 shows a summary of the number of models which met the initial criteria for fit to both the ID and the TEST data in the three configurations.

The first number in Table 13 is the total number of models for which a set of ID and TEST data met the valid model criteria. This number includes instances where the same set of ID data generated the same model, but was validated with several different sets of the TEST data, indicating the estimated models are capable of predicting more than simply one other set of maneuvers. The number of unique models estimated in each configuration is shown in the second row of Table 13. The highest number of valid models was estimated in the Jib configuration because the most usable data was in that configuration. While on paper a comparable amount of data was collected in the Genoa configuration, post-processing revealed that much of it was not suitable for linear system identification with OSLS because many maneuvers did not exhibit a roll response because the wind speed was so light and trimmed roll angles were very small. A different model structure or estimation algorithm would be necessary to use this data for system identification. Only one model was estimated in the Reef configuration as there were very few maneuvers suitable for system identification. The high wind and wave conditions that the boat operates in for the Reef configuration caused some of the electrical connections on the data acquisition system to come loose, resulting in a substantial loss of data in this configuration. 


\subsubsection{Modal Analysis of Estimated Linear Models}

Two differing model characteristics are observed in the set of estimated models. The first is a model which exhibits two second order modes and one first order mode, and is referred to herein as a Type A model. The second is a model which exhibits one second order and three first order modes, and is referred to herein as a Type B model. The individual modes of each model are sorted by natural frequency and labeled by a number designation followed by ' $A$ ' or ' $\mathrm{B}$ ' depending upon which model set they have come from, and an ' $\mathrm{S}$ ' denoting a second order mode or an ' $F$ ' denoting a first order modes.

Table 14 shows the mean of the first order modes for all estimated models. There is an overall shift towards the right in all the first order modes from the Jib configuration to the Genoa configuration. This shift to the right means each first order mode has a higher time constant, which may be explained by the physical differences in the aerodynamic forces in the two sail configurations and the amount of energy they are able to harvest from the wind.

In order to perform the modal analysis which is relevant for control system design, the complex plane was divided into three regions, Stable (S), Marginally Stable (MS), and Unstable (US). Second order Mode 1A.S and Mode 4B.S represent the mode with highest damping and transitions through each of the three stability regions. With a subset of the Jib models representing a better fit to the ID data with an $R^{2}>0.88$, the eigenvector modal analysis provides insight into why some modes may move through all three regions. For Mode 1A.S this analysis reveals a strong dependence upon trimmed roll angle. All Modes 1 A.S with $\Phi>12^{\circ}$ fall into the

Table 14: Mean locations of all real roots for Jib and Genoa.

\begin{tabular}{|c|c|c|c|c|}
\hline & Mode 3A.F & Mode 1B.F & Mode 2B.F & Mode 3B.F \\
\hline Jib Configuration & -0.219 & -0.294 & -0.051 & 0.015 \\
\hline Genoa Configuration & 0.034 & -0.158 & -0.006 & 0.094 \\
\hline$($ Genoa - Jib $)$ & 0.253 & 0.452 & 0.045 & 0.079 \\
\hline
\end{tabular}


S region. For $\Phi>10^{\circ}$, roll angle and roll rate dominate the modal behavior, and for $\Phi<8^{\circ}$ surge velocity and yaw rate dominate the modal behavior. The transition region, which for the test yacht is between 8 and 10 degrees of roll, should be well defined by eigenvector analysis before control system design is undertaken for other sailing yachts. It would be expected that two robust controllers could be gain scheduled with one model governing the low heel angle (US, MS) region and another model governing the high heel angle (S) region. Another possibility would be to make use of adaptive control strategies.

All the estimated models also exhibit the same first order mode, 3A.F and 1B.F. This first order mode represents the shortest time constant, 5 seconds in the Jib configuration, of the first order modes observed in the estimated models. Some of the models placed Mode 3A.F in the MS or US region. Eigenvector analysis showed that all stable modes 3A.F occurred with a surge contribution, $M_{u}^{\prime}<0.4$, and a sway contribution, $M_{v}^{\prime}>0.2$. If these criterion are not met for a model, this mode must be treated as unstable for control system design.

The distinguishing characteristic between the Type A and Type B models is the low energy second order Mode 2A.S which breaks into two first order modes, 2B.F, and 3B.F. Mode 2A.S is a very low damped, and therefore low energy mode for which the maneuvers performed during this study were not very appropriate to excite. Mode 2B.F is always stable with a time constant of 20 seconds for the Jib configuration, and 167 seconds for the Genoa configuration. Mode 3B.F can be unstable. The eigenvector analysis reveals that surge is the greatest contributor to this mode with an $M_{u}^{\prime}>0.4$ for all models.

As these are the first linear sailing yacht models to be published in the open literature, the modal analysis presented in Section 8.4.1 has resulted in fundamental insight into how the sailing yacht behaves as a dynamic system. When considering model choices for control system design, both the Jib and Genoa configurations support the hypothesis that roll angle, which is closely related to wind speed, is the most important cause of differences in the model modal characteristics. This information is invaluable for control system design as it sets the range of what to expect for the sailing yacht. Robust linear controllers may be implemented using the linear model structure. Because the sailing yacht has demonstrated unstable modes, controllers may be used to guarantee the stability of the system, but not necessarily optimal performance. 


\subsection{Recommendations for Future Work}

This project has been a first step towards utilizing physics based models for advanced control system design for autonomous sailing yachts. To carry the project further there are several recommendations for future work in this field. The first few paragraphs below describe ways in which the analysis performed here could be improved with the current test platform through both more data collection and system identification. The last recommendations address developments required for the realization of a real autonomous sailing yacht ocean sensing platform.

\subsubsection{Further Data Collection}

Collecting more data in both the Genoa and Reef configurations using the same data acquisition system, maneuvers, linear modeling, and system identification approach outlined in this document would help further support the conclusions made in the Jib configuration. Both of these conditions would likely require an additional crew member to ensure the success of the experiments. More data in the two configurations would allow more specific conclusions about the observed modes. Collecting data at higher acquisition rate than $5 \mathrm{~Hz}$ may improve the quality of models estimated. In the Genoa configuration, the future data collection should focus on higher wind speeds than that which has currently been collected. A substantial portion of the collected data did not capture the full surge response of the boat. In order to improve the current test procedure, the surge velocity should be fed back to the helmsman to ensure sufficient time is allowed to capture the full response.

The full-scale tests and maneuvers were designed to maintain constant apparent wind angles and boat speeds, and left the roll degree of freedom uncontrolled during tests. It would have been possible, especially in the upwind condition, to depower the sail plan by letting the mainsail luff slightly and perform tests at controlled, differing roll angles for the same apparent wind angle. Such tests would no doubt result in a range of boat speeds as well, but they have potential to aid in further insight into Mode 2A.S and the difference between the Type A and Type B models. New maneuvers need to be designed to better characterize Mode 2A.S.

The downwind sailing trim state was not included in this study, and would likely not require a model with the roll degree of freedom, at least for the test yacht sail plan (which does not include a dedicated downwind sail). However, many yachts carry a separate downwind sail and 
repeating the testing and model identification process would be interesting, but likely not yield any practical results. Downwind sails can be a hassle, even for an experienced crew, and it is unlikely an autonomous yacht would have a dedicated downwind sail unless it used a solid wing sail rather than flexible sails.

\subsubsection{Modifications to the Current Model}

Wind has not been included as part of the linear model in this work. It is possible to add wind as an external disturbance, which would represent the next step in linear model development. The current set of data contains wind gusts and wind shifts, and these segments could be treated as maneuvers and the same linear system identification performed to characterize the effects of wind on the behavior of the yacht. Including wind would likely increase performance of controllers, although the current model is likely satisfactory for control system design and performance. The only way to answer the question whether wind should be included in the model would be to estimate models with and without, and gauge their performance through real control system implementation on test yachts.

\subsubsection{Alternate Model Estimation Strategies}

The estimated state space models could be used to perform real parameter estimation of lumped aerodynamic and hydrodynamic force and moment derivatives. Full parameter estimation would likely shed even more light on the dynamic behavior of the yacht, and enable better control system design through a more thorough understanding of the physics behind the model.

The author experimented quite a bit with using artificial neural networks on small portions of the full scale data. While not included in this work, the author believes neural networks have great potential for model identification and control of sailing yacht dynamic systems. Approaches used in aerospace to characterize stability and control derivatives using neural networks would be a good place to start in designing neural networks for sailing yacht model identification. Almost all of the 20 hours of sailing motion data collected for this work would be suitable for neural network system identification. 


\subsubsection{Autonomous Sailing Platform Development}

Current autonomous sailing platforms exist for competitions such as the World Robotic Sailing Competition and SailBot. The modeling and system identification presented in this work could easily be applied to these platforms as they already have the necessary sensors and electronics. With estimated linear models advanced multi-input multi-output controllers could be tested on these platforms. One major advantage the linear models of this work offer to competitors is the ability to use the sail to control the roll angle of the yacht, an important consideration for the safety of an autonomous boat attempting an ocean crossing.

In some cases it may prove a better design choice for a purpose built autonomous sailing yacht to have a solid wing sail rather than soft sails in the interest of durability. The models and techniques outline in this work could easily be adapted to model a wing rather than a sail. The wing would give more consistent performance than the soft sails because it's shape is not variable which would make the model identification process more accurate. Considerations regarding the wind should include safety and the ability to depower in heavy weather.

In the interest of fully developing an autonomous sailing platform for remote sensing, it would be extremely valuable to start from a clean sheet of paper and design a purpose built yacht for autonomous operation. Outfitting the yacht with similar sensors, the current techniques could be applied very quickly and control system design and testing could begin. The big advantage in designing a purpose built yacht is that all mass parameters, center of gravity, and aerodynamic and hydrodynamic centers of effort would be much better known than they were for this test yacht platform and would open the door for real, physics-based non-linear parameter estimation from sailing data. Aerodynamic and hydrodynamic parameters could be measured for the purpose built yacht and compared to parameter estimation results. In addition to proving useful for control system design, non-linear parameter estimation would be an invaluable tool for developing full simulators for training purposes. 
The study of sailing yacht dynamics with system identification techniques has proven very insightful and is ripe for future developments. The world's oceans are still vastly unexplored and scientists are actively trying to better understand and characterize the effects of pollution and coastal inundation, to name a few, on not just marine ecosystems but on human society and the world's economy. Autonomous sailing platforms have great potential to give new kinds of data to scientists through their unique advantages over existing sensing platforms. 


\section{References}

1. Abkowitz, M. A. (1980). Measurement of Hydrodynamic Characteristics from Ship Maneuvering Trials by System Identification. SNAME Transactions, 88, 283-318.

2. Akimoto, H., Kim, Y.-H., Park, J.-C., \& Jeong, S.-m. (2012). CFD simulation of a sailing boat hull in maneuvering condition. Paper presented at the MTS/IEEE OCEANS 2012 Yeosu, South Korea.

3. Alves, J., Ramos, T., \& Cruz, N. (2008). A reconfigurable computing system for an autonomous sailboat. Paper presented at the International Robotic Sailing Conference, Breitenbrunn, Austria.

4. Amin, S. M., Gerhart, V., \& Rodin, E. Y. (1997). System Identification via Artificial Neural Networks: Applications to On-line Aircraft Parameter Estimation. Paper presented at the World Aviation Congress, Anaheim, CA.

5. Anderson, J. D. J. (2007). Fundamentals of Aerodynamics, $4^{\text {th }}$ Ed. New Work: McGrawHill.

6. Augier, B., Bot, P., Hauville, F., \& Durand, M. (2013). Dynamic behaviour of a flexible yacht sail plan. Ocean Engineering, 66(2013), 32-43.

7. Azcueta, R. (2002, December). RANSE simulations for sailing yachts including dynamic sinkage \& trim and unsteady motions in waves. Paper presented at the High Performance Yacht Design Conference, Auckland, NZ.

8. Bevington, P. R., \& Robinson, D. K. (2003). Data Reduction and Error Analysis for the Physical Sciences (3rd ed.). New York: McGraw Hill.

9. Bhattacharyya, S. K., \& Haddara, M. R. (2006). Parametric Identification for Nonlinear Ship Maneuvering. Journal of Ship Research, 50(3), 197-207.

10. Billings, S. A. (1980). Identification of nonlinear systems- a survey. Control Theory and Applications, IEEE Proceedings D, 127(6), 272-285.

11. Binns, J., Hochkirch, K., De Bord, F., \& Burns, I. (2008, 2-4 December, 2008). The development and use of sailing simulation for IACC starting manoeuvre training. Paper presented at the 3rd High Performance Yacht Design Conference, Auckland, NZ.

12. Blanke, M., \& Christensen, A. (1993). Rudder Roll Damping Autopilot Robustness due to Sway-Yaw-Roll Couplings. Paper presented at the 10th International Ship Control Systems Symposium (SCSS '93), Ottawa, Canada.

13. Ciortan, C., \& Guedes Soares, C. (2007). Computational study of sail performance in upwind condition. Ocean Engineering, 34(16), 2198-2206.

14. Cleveland, W. S. (1979). Robust Locally Weighted Regression and Smoothing Scatterplots. Journal of the American Statistical Association, 74(368), 829-836.

15. Cruz, N. A., \& Alves, J. C. (2008). Autonomous sailboats: An emerging technology for ocean sampling and surveillance. Paper presented at the IEEE/ OCEANS 2008, Quebec City, Canada. 
16. Das, S., Kuttieri, R. A., Sinha, M., \& Jategaonkar, R. V. (2010). Neural Partial Differential Method for Extracting Aerodynamic Derivatives from Flight Data. AIAA Journal of Guidance, Control, and Dynamics, 33(2), 376-384.

17. de Ridder, E., Vermeulen, K., \& Keuning, J. (2004). A Mathematical Model for the Tacking Maneuver of a Sailing Yacht. Paper presented at the HISWA Symposium on Yacht Design and Yacht Construction.

18. Elkaim, G. H. (2002). System Identification for Precision Control of a WingSailed GPSGuided Catamaran. (Ph.D.), Stanford University.

19. Elkaim, G. H. (2009). System identification-based control of an unmanned autonomous wind-propelled catamaran. Control Engineering Practice, 17(1), 158-169. doi: 10.1016/j.conengprac.2008.05.014

20. Fossati, F., \& Muggiasca, S. (2010). Numerical modelling of sail aerodynamic behavior in dynamic conditions. Paper presented at the 2nd International Conference on Innovation in High Performance Sailing Yachts, Lorient, France.

21. Fossen, T. I. (2011). Handbook of Marine Craft Hydrodynamics and Motion Control: John Wiley \& Sons.

22. Fossen, T. I., Sagatun, S. I., \& Sørensen, A. J. (1996). Identification of dynamically positioned ships. Control Engineering Practice, 4(3), 369-376.

23. Gentry, A. (1995). Ranger 23 Boatspeed Polars: What are they and how to use them. Retrieved from www.arvelgentry.com

24. Gerritsma, J., Moeyes, G., \& Onnink, R. (1977). Test Results of a Systematic Yacht Hull Series. Paper presented at the 5th HISWA Symposium, Amsterdam.

25. Graf, K. (Olaf Muller, March). Photogrammetric Investigation of the Flying Shape of Spinnakers in a Twisted Flow Wind Tunnel. Paper presented at the The 19th Chesapeake Sailing Yacht Symposium, Annapolis, MD.

26. Graf, K., Boehm, C., \& Renzsch, H. (2009, March). CFD- and VPP-Challenges in the Design of the New AC90 Americas Cup Yacht. Paper presented at the The 19th Chesapeake Sailing Yacht Symposium, Annapolis, MD.

27. Graf, K., \& Bohm, C. (2005). A New Velocity Prediction Method for Post-Processing of Towing Tank Test Results. Paper presented at the 17th Chesapeake Sailing Yacht Symposium, Annapolis, MD.

28. Graf, K., Pelz, M., Bertram, V., \& Soding, H. (2007, March). Added Resistance in Seaways and its Impact on Yacht Performance. Paper presented at the 18th Chesapeake Sailing Yacht Symposium, Annapolis, MD.

29. Hansen, H. (2006). Enhanced Wind Tunnel Techniques and Aerodynamic Force Models for Yacht Sails. (Ph.D.), University of Auckland, Auckland.

30. Hansen, H., Jackson, P. S., \& Hochkirch, K. (2003, 17-18 September, 2003). Real-Time Velocity Prediction Program for Wind Tunnel Testing. Paper presented at the International Conference on the Modern Yacht, Southampton, UK. 
31. Jacquin, E., Roux, Y., Guillerm, P.-E., \& Alessandrini, B. (2005). Toward numerical $V P P$ with the full coupling of hydrodynamic and aerodynamic solvers for ACC yacht. Paper presented at the 17th Chesapeake Sailing Yacht Symposium, Annapolis, MD.

32. Jategaonkar, R. V. (2006). Flight Vehicle System Identification: A Time Domain Methodology (Vol. 216). Reston, VA: AIAA.

33. Jategaonkar, R. V., Fischenberg, D., \& von Gruenhagen, W. (2004). Aerodynamic Modeling and System Identification from Flight Data- Recent Applications at DLR. Journal of Aircraft, 41(4), 681-691.

34. Johnson, B., Miles, J. C., Lasher, W. C., \& Womack, J. (2009). Operator Guidance Based on Assessing the Wind-Heel Angle Relationship of Traditionally-Rigged Sailing Vessels.

35. Kerwin, J. (1975). A velocity prediction program for ocean racing yachts: Massachusetts Institute of Technology, Dept. of Ocean Engineering, Cambridge, MA

36. Kerwin, J. (1978). A Velocity Prediction Program for Ocean Racing Yachts revised to February 1978. Massachusetts Institute of Technology, Dept. of Ocean Engineering, Cambridge, MA.

37. Keuning, J. A., \& Katgert, M. (2008). A Bare Hull Resistance Prediction Method Derived from the Results of the Delft Systematic Yacht Hull Series Extended to Higher Speeds. Paper presented at the Intl. Conference of Innovations in High Performance Sailing Yachts, Lorient.

38. Kim, W.-J., Yoo, J., Chen, Z., Rhee, S. H., Chi, H.-R., \& Ahn, H. (2009). Hydro- and aerodynamic analysis for the design of a sailing yacht. Journal of Marine Science and Technology, 15(3), 230-241. doi: 10.1007/s00773-010-0088-8

39. Kwakernaak, H., \& Sivan, R. (1972). Linear Optimal Control Systems. New York: John Wiley \& Sons.

40. Le Pelley, D., \& Modral, O. (2008). V-SPARS: A combined Sail and Rig Shape Recognition System Using Imaging Techniques. Paper presented at the $3{ }^{\text {rd }}$ High Performance Yacht Design Conference, Auckland, NZ.

41. Letcher Jr, J. (1974). Handicapping rules and performance of sailing yachts. Paper presented at the 1st Chesapeake Sailing Yacht Symposium.

42. Linse, D. J., \& Stengel, R. F. (1993). Identification of Aerodynamic Coefficients Using Computational Neural Networks. AIAA Journal of Guidance, Control, and Dynamics, 16(6), 1018-1025.

43. Ljung, L. (1999). System Identification: Theory for the User. Upper Saddle River, NJ: Prentice Hall.

44. Masayuma, Y., \& Fukasawa, T. (2011). Tacking Simulation of Sailing Yachts with New Model of Aerodynamic Force Variation During Tacking Maneuver. SNAME Journal of Sailboat Technology, 2011-01.

45. Masayuma, Y., Nakamura, I., Tatano, H., \& Takagi, K. (1993, Jan 29). Dynamic Performance of Sailing Cruiser by Full-Scale Sea Tests. Paper presented at the 11th Chesapeake Sailing Yacht Symposium, Annapolis, MD. 
46. Masuyama, Y., Fukasawa, T., \& Sasagawa, H. (1995). Tacking simulation of sailing yachts-numerical integration of equations of motion and application of neural network technique. Paper presented at the 12th Chesapeake Sailing Yacht Symposium, Annapolis, MD.

47. Masuyama, Y., Tahara, Y., Fukasawa, T., \& Maeda, N. (2009). Database of sail shapes versus sail performance and validation of numerical calculations for the upwind condition. Journal of Marine Science and Technology, 14(2), 137-160.

48. Masuyuma, Y., Nakamura, I., Hisayoshi, T., \& Takagi, K. (1993). Dynamic Performance of Sailing Cruiser by Full-Scale Sea Tests. Paper presented at the 11th Chesapeake Sailing Yacht Symposium, Annapolis, MD.

49. Miller, P., Brooks, O., \& Hamlet, M. (2009). Development of the USNA SailBots (ASV) Paper presented at the 2nd Internationl Robotic Sailing Conference, Matosinhos, Portugal.

50. Miyata, H., Akimoto, H., \& Hiroshima, F. (1997). CFD performance prediction simulation for hull-form design of sailing boats. Journal of Marine Science and Technology, 2(4), 257-267.

51. Miyata, H., \& Lee, Y. (1999). Application of CFD simulation to the design of sails. Journal of Marine Science and Technology, 4(4), 163-172.

52. Morelli, E. A., \& Klein, V. (2005). Application of System Identification to Aircraft at NASA Langley Research Center. Journal of Aircraft, 42(1), 12-24.

53. Neal, M., Sauze, C., Thomas, B., \& Alves, J. C. (2009, 9 July). Technologies for Autonomous Sailing: Wings and Wind Sensors. Paper presented at the 2nd Internationl Robotic Sailing Conference, Matosinhos, Portugal.

54. Ogata, K. (1997). Modern Control Engineering (3rd Ed.). Upper Saddle River, NJ: Prentice-Hall.

55. Paris, A., \& Bonner, M. (2004). Nonlinear Model Development from Fllight-Test Data for F/A-18E Super Hornet. Journal of Aircraft, 41(4), 692-702.

56. Parolini, N., \& Quarteroni, A. (2007). Modelling and numerical simulation for yacht engineering. Paper presented at the 26th International Symposium on Naval Hydrodynamics.

57. Petres, C., Romero-Ramirez, M.-A., Plumet, F., \& Alessandrini, B. (2011). Modeling and reactive navigation of an autonomous sailboat. Paper presented at the International Conference on Intelligent Robots and Systems, San Francisco, CA.

58. Peyada, N. K., \& Ghosh, A. K. (2009). Aircraft parameter estimation using a new filtering technique based upon a neural network and Gauss-Newton method. The Aeronautical Journal, 113(1142), 243-252.

59. Raisinghani, S. C., Bhaskar Reddy, V. S., \& Ghosh, A. K. (1999). Parameter estimation of an augmented aircraft using neural networks. Paper presented at the AIAA Atmospheric Flight Mechanics Conference and Exhibit, Portland 
60. Richardt, T., Harries, S., \& Hochkirch, K. (2005). Maneuvering simulations for ships and sailing yachts using FRIENDSHIP-Equilibrium as an open modular workbench. Paper presented at the International Euro-Conference on Computer Applications and Information Technology in the Maritime Industries, Hamburg.

61. Roskam, J. (2007). Airplane Flight Dynamics and Automatic Flight Controls; Part I. Lawrence, KS: DARcorporation.

62. Ross, A. (2008). Nonlinear Maneuvering Models for Ships: A Lagrangian Approach. (Ph.D.), Norwegian University of Science and Technology.

63. Roux, Y., Huberson, S., Hauville, F., Boin, J., Guilbaud, M., \& Ba, M. (2002). Yacht performance prediction: towards a numerical VPP. Paper presented at the High Performance Yacht Design Conference, Auckland, NZ.

64. Rynne, P. F., \& von Ellenrieder, K. D. (2010). Development and Preliminary Experimental Validation of a Wind- and Solar-Powered Autonomous Surface Vehicle. IEEE Journal of Oceanic Engineering, 35(4).

65. Son, K. H., \& Nomoto, K. (1982). On the Coupled Motion of Steering and Rolling of a High-speed Container Ship. Naval Architect of Ocean Engineering, Japan, 150(20), 7383.

66. Stelzer, R., Proll, T., \& John, R. (2007). Fuzzy logic control system for autonomous sailboats. Paper presented at the Fuzzy Systems Conference, London.

67. Stengel, R. F. (2004). Flight Dynamics. Princeton, NJ: The Princeton University Press.

68. Taylor, J. R. (1997). An Introduction to Error Analysis. Sausalito, CA: University Science Books.

69. Theil, H. (1958). Economic Forecasts and Policy. Amsterdam: North-Holland Publishing Co.

70. Tiano, A., Zirilli, A., Yang, C., \& Xiao, C. (2001). A neural autopilot for sailing yachts.

71. Tischler, M. B., \& Remple, R. K. (2006). Aircraft and Rotorcraft System Identification. Reston, VA: AIAA.

72. Viola, I. M., \& Flay, R. G. J. (2012). Sail Aerodynamics: On-Water Pressure Measurements on a Downwind Sail. Journal of Ship Research, 56(4), 197-206.

73. Wang, L. (2012). Dynamic Analysis of a Microgrid System for Supplying Electrical Loads in a Sailing Boat. Paper presented at the Power and Energy Society General Meeting, San Diego, CA.

74. Xiao, L., \& Jouffroy, J. (2011). Modeling and nonlinear heading control for sailing yachts. Paper presented at the MTS/IEEE OCEANS 2011, Waikoloa, HI.

75. Yang, B., Xiao, L., \& Jouffroy, J. (2011). A control-theoretic outlook at the no-go zone in sailing vessels. Paper presented at the MTS/IEEE OCEANS 2011, Waikoloa, HI.

76. Yoo, J., \& Kim, H. (2006). Computational and experimental study on performance of sails of a yacht. Ocean Engineering, 33(10), 1322-1342. 
77. Yoon, H. K., \& Rhee, K. P. (2003). Identificaiton of hydrodynamic coefficients in ship maneuvering equations of motion by Estimation-Before-Modeling technique. Journal of Ocean Engineering, 30(18), 2379-2404.

78. Zhou, K., \& Doyle, J. C. (1997). Essentials of Robust Control: Prentice Hall. 


\section{Appendix A : Sailing Terminology}

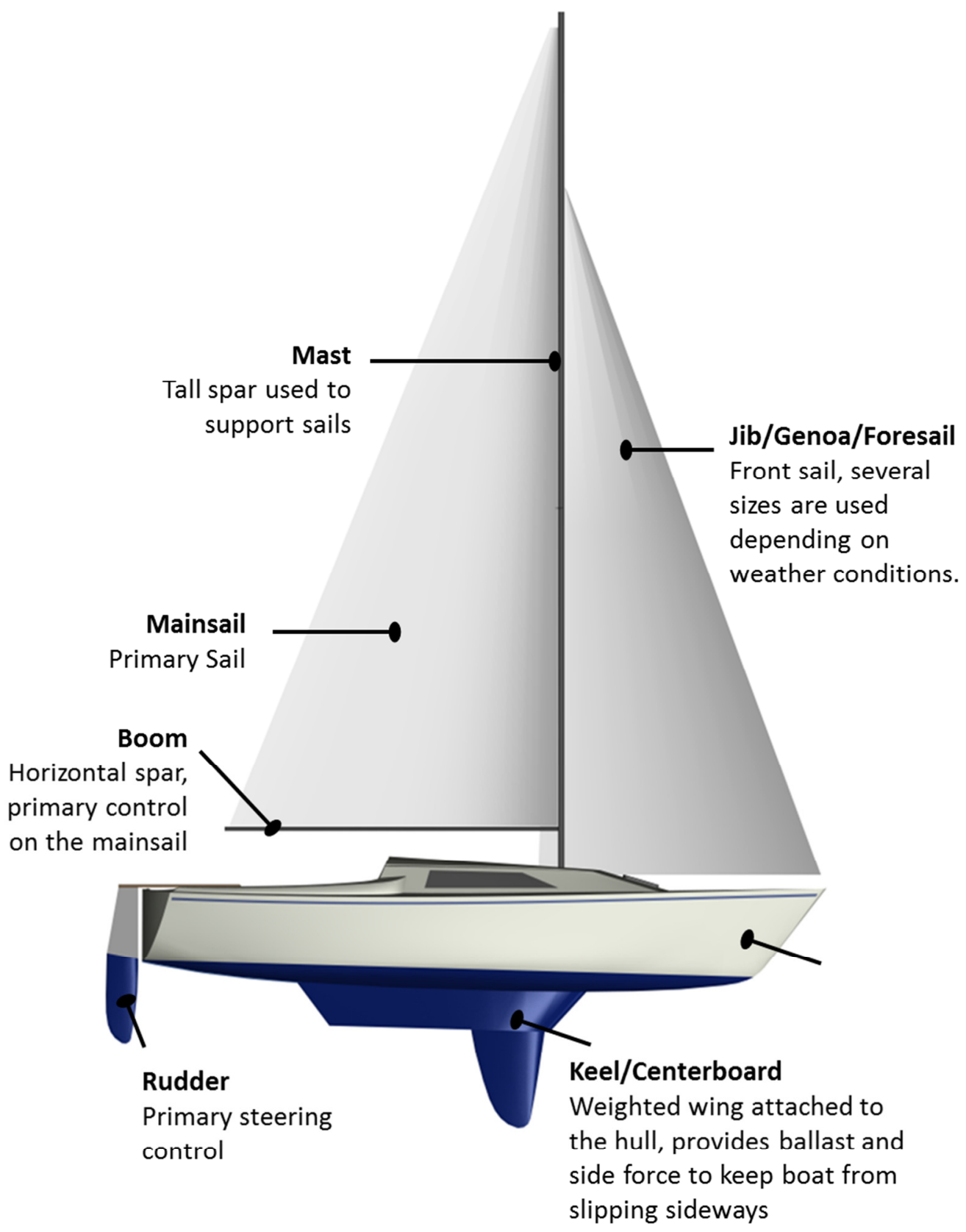

Figure 81: Basic Parts of a Monohull sailing Yacht 


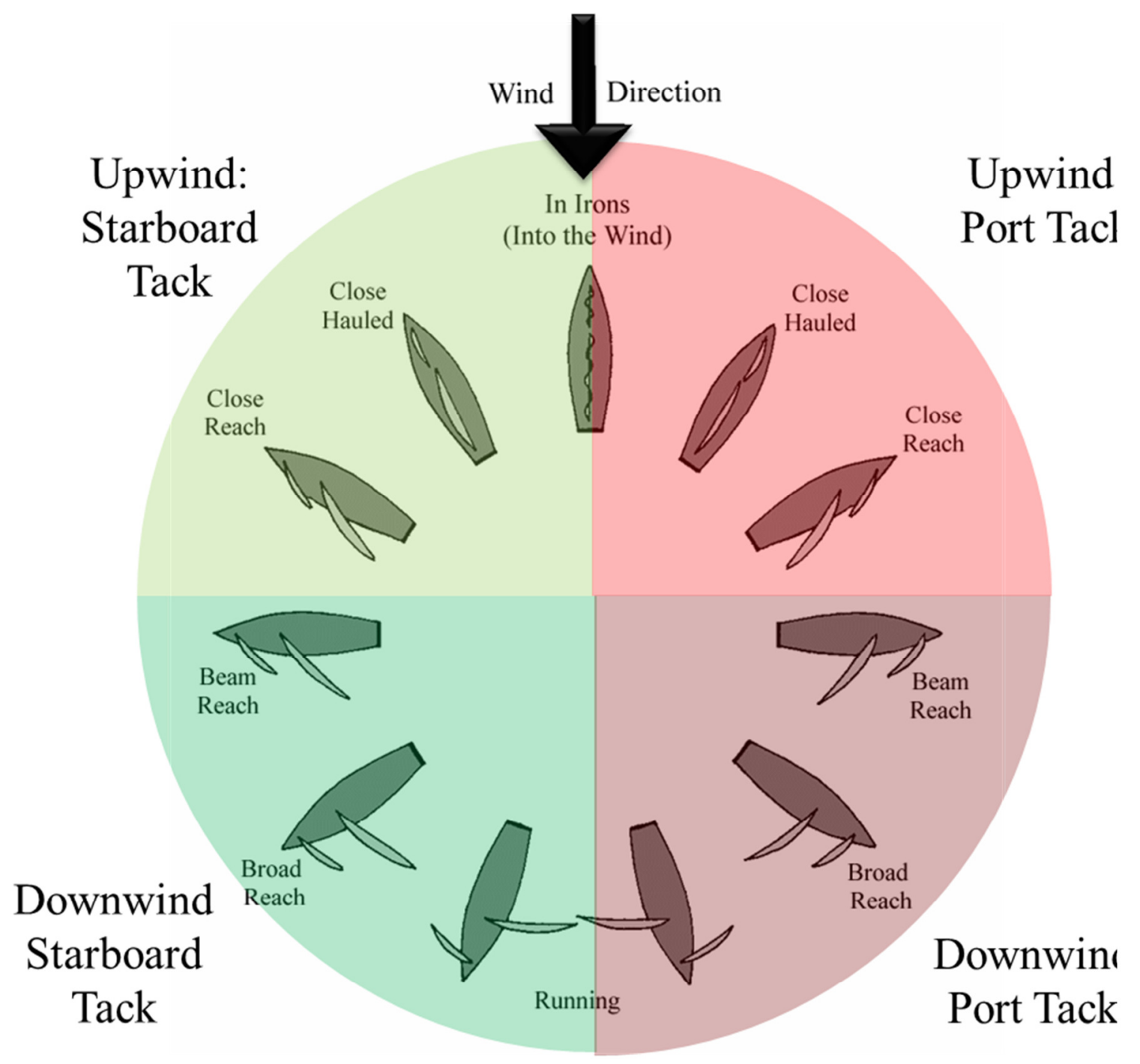

Figure 82: Points of Sail

Bulb Keel - A keel which usually has a high aspect ratio foil with a weighted bulb at the bottom, these are used to place the ballast as deep as possible. These are generally found on offshore craft because they have such deep draft.

Catamaran - Boat with two canoe like hulls rather than a single hull. They are connected by strong spars between the hulls, and generally are designed to sail with one hull out of the water to minimize skin friction drag. Catamarans usually have one rudder on each hull, and are harder to turn through the wind during a tack because of the underwater geometry of the hulls.

Downwind - Sailing a broad reach or running course, sails are generally let out far from centerline, and, if equipped, a spinnaker is used instead of a jib or genoa.

Foretriangle - The triangle formed by the forestay, mast, and deck of a sailing yacht. 
Forestay - Part of the standing rigging of a sailing yacht that extends from the bow to a point anywhere from three-quarters of the distance up the mast to the top of the mast. Its purpose is to keep the mast from falling backwards. It is commonly also used as the point where the foresail may be hoisted.

Genoa - A foresail which is larger than the foretriangle. The size of the genoa is denoted as a percentage of the length of the bottom of the foretriangle. So, the foot of a $150 \%$ genoa will be $150 \%$ of the length from the bow to mast.

Handicapping System - A system by which yachts of different sizes and configurations may race against one another and have their times altered based on a variety of factors to allow 'equal' competition. Some handicapping systems include Performance Handicap Racing Fleet (PHRF), Portsmouth, IMS, and IRC.

Head to Wind, Irons - A situation where the yacht has its bow pointing directly into the wind. No thrust can be generated by soft sails in this situation, resulting in almost no maneuverability.

$\boldsymbol{J i b}$ - A foresail which is generally the same size or smaller than the foretriangle.

Jibe - Maneuver during which a sailing yacht changes tacks by passing its stern through the wind.

Keel - A hydrodynamic lifting surface attached to the bottom of a sailing yacht to counteract the force of the wind pushing the boat to leeward. Keels often contain the lead ballast that stabilizes the boat in roll.

Leeward - The direction downwind from a point of reference.

Luffing - A phenomena that occurs in soft flexible sails when they are pointed too far into the wind. It could be a small luff at the front of the sail or the whole sail flapping and anywhere in between.

Main Sail - The primary sail on a sailing yacht which is hoisted up the mast.

Match Race - A race between two boats of the same class, the most famous is the America's Cup.

Monohull - A sailing yacht with one hull.

Port - The left side of a boat.

Port Tack - Sailing with the sails on the starboard (right) side of the boat, wind is coming from the port side of the boat, meaning port is windward and starboard is leeward.

$\boldsymbol{R i g}$ - All the components that hold up the sails including the mast, stays, and boom

Rudder - Primary control on a sailing yacht used for directional control. The rudder can be connected to a tiller or to a wheel, which is what the helmsman operates.

Sheet - A line (rope) attached to a sail which is used for primary sail control. 
Starboard - The right side of a boat.

Starboard Tack - Sailing with the sails on the port (left) side of the boat, the wind is coming from the starboard side of the boat, meaning starboard is windward and port is leeward.

Tack - A maneuver during which a sailing yacht changes tack by passing the bow through the wind. Also defines the alignment of the sailing yacht with the wind, see Port Tack and Starboard Tack.

Trim - A yacht sailing in trim means the sails have been adjusted by their various controls to suit the current wind conditions.

Trimaran - A boat with three hulls, the center primary hull with two outriggers.

Upwind - Sailing a close hauled course, sails are generally pulled in close to centerline and boat is heeled over and sailing with leeway

Velocity Made Good (VMG) - A term which refers to the component of the total boat velocity that is in the direction of the next mark or waypoint.

Windward - The direction upwind from a point of reference.

Wingsail - A sail with a 2D profile, usually an airfoil shape. These can be solid wings or fabric stretched over substructure. 


\section{Appendix B : Delft Systematic Yacht Hull Series}

The following is a list of symbols that is only applicable to this Appendix and is not to be applied to any other variables in this paper.

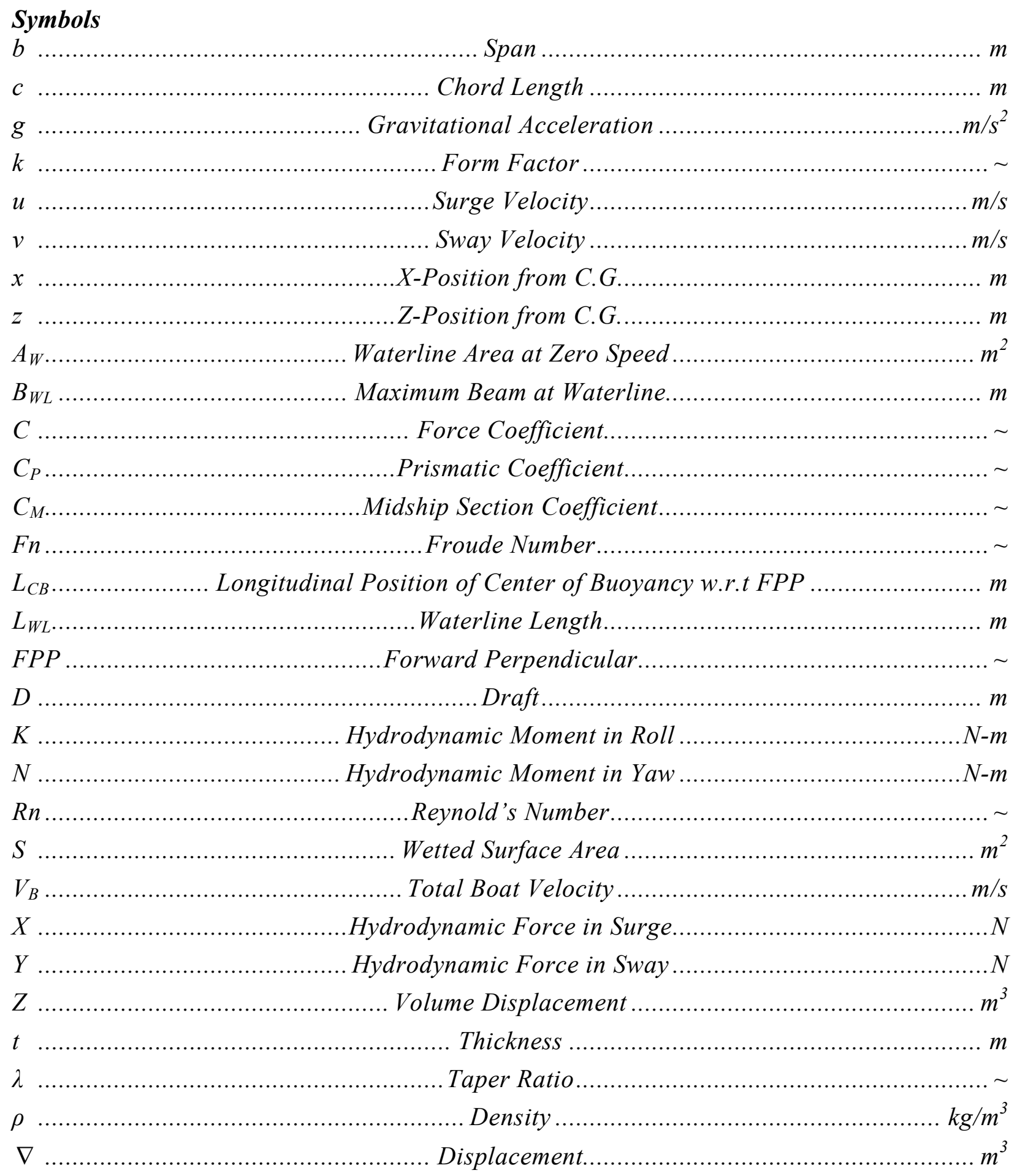




\section{Subscripts}

$c$

Canoe Body

$k$ Keel

$r$ Rudder

CLR Center of Lateral Resistance

F Frictional Resistance

$I$ Induced Resistance

$R$ Residuary Resistance

V Viscous Resistance

$W L$. Waterline

The waterline length is calculated as $70 \%$ of the true waterline length. All equations and tables of polynomial fitting coefficients are taken from (de Ridder, Vermeulen, \& Keuning, 2004) unless referenced otherwise.

The expression for total surge force due to forward velocity is:

$$
-X_{H}=\underbrace{X_{R_{c}}+X_{F_{c}}+X_{V_{k}}+X_{V_{v}}}_{\text {Upright Resistance }}+\underbrace{X_{R \phi_{c}}+X_{F \phi_{c}}+X_{R \phi_{k}}}_{\text {Added Resistance due to heel }}+\underbrace{X_{v} \sin \left(\frac{-v}{u}\right)-X_{I} \cos \left(\frac{-v}{u}\right)}_{\text {Forces due to Side Velocity (leeway) }}
$$

The frictional resistance of the hull is given by:

$$
X_{F_{c}}=1 / 2 \rho u^{2} S_{c}\left(\frac{0.075}{(\log (R n)-2)^{2}}\right)
$$

The residuary resistance of the canoe body is given by Eq. (B.3) and Table 15 (Keuning \& Katgert, 2008):

$$
X_{R_{c}}=\left\{a_{0}+\left(a_{1} \frac{L_{C B}}{L_{W L}}+a_{2} C_{P}+a_{3} \frac{\nabla_{c}^{2 / 3}}{A_{W}}+a_{4} \frac{B_{W L}}{L_{W L}}+a_{5} \frac{L_{C B}}{L_{C F}}+a_{6} \frac{B_{W L}}{D_{c}}+a_{7} C_{M}\right)\right\} \nabla_{c} \rho g \cdot \frac{\nabla_{c}^{2 / 3}}{L_{W L}}
$$


Table 15: DSYHS Coefficients for Residuary Resistance of Cano Body

\begin{tabular}{|c|c|c|c|c|c|c|c|c|c|c|c|c|}
\hline Fn & 0.10 & 0.15 & 0.20 & 0.25 & 0.30 & 0.35 & 0.40 & 0.45 & 0.50 & 0.55 & 0.60 & 0.65 \\
\hline $\mathbf{a 0}$ & -0.0005 & -0.0003 & -0.0002 & -0.0009 & -0.0026 & -0.0064 & -0.0218 & -0.0388 & -0.0347 & -0.0361 & 0.0008 & 0.0108 \\
\hline a1 & 0.0023 & 0.0059 & -0.0156 & 0.0016 & -0.0567 & -0.4034 & -0.5261 & -0.5986 & -0.4764 & 0.0037 & 0.3728 & -0.1238 \\
\hline a2 & -0.0086 & -0.0064 & 0.0031 & 0.0337 & 0.0446 & -0.125 & -0.2945 & -0.3038 & -0.2361 & -0.296 & -0.3667 & -0.2026 \\
\hline a3 & -0.0015 & 0.007 & -0.0021 & -0.0285 & -0.1091 & 0.0273 & 0.2485 & 0.6033 & 0.8726 & 0.9661 & 1.3957 & 1.1282 \\
\hline a4 & 0.0061 & 0.0014 & -0.007 & -0.0367 & -0.0707 & -0.1341 & -0.2428 & -0.043 & 0.4219 & 0.6123 & 1.0343 & 1.1836 \\
\hline a5 & 0.001 & 0.0013 & 0.0148 & 0.0218 & 0.0914 & 0.3578 & 0.6293 & 0.8332 & 0.899 & 0.7534 & 0.323 & 0.4973 \\
\hline a6 & 0.0001 & 0.0005 & 0.001 & 0.0015 & 0.0021 & 0.0045 & 0.0081 & 0.0106 & 0.0096 & 0.01 & 0.0072 & 0.0038 \\
\hline a7 & 0.0052 & -0.002 & -0.0043 & -0.0172 & -0.0078 & 0.1115 & 0.2086 & 0.1336 & -0.2272 & -0.3352 & -0.4632 & -0.4477 \\
\hline
\end{tabular}

The residuary resistance of the keel is given by Eq. (B.4) with coefficients from Table 16:

$$
X_{R_{k}}=\left(A_{0}+A_{1} \frac{D_{c}+b_{k}}{B_{W L}}+A_{2} \frac{D_{c}+Z_{k}}{\nabla_{k}^{1 / 3}}+A_{3} \frac{\nabla_{c}}{\nabla_{k}}\right) \nabla_{k} \rho g
$$

Table 16: DSYHS Coefficients for Residuary Resistance of Keel

\begin{tabular}{|c|c|c|c|c|c|c|c|c|c|}
\hline Fn & $\mathbf{0 . 2 0}$ & $\mathbf{0 . 2 5}$ & $\mathbf{0 . 3 0}$ & $\mathbf{0 . 3 5}$ & $\mathbf{0 . 4 0}$ & $\mathbf{0 . 4 5}$ & $\mathbf{0 . 5 0}$ & $\mathbf{0 . 5 5}$ & $\mathbf{0 . 6 0}$ \\
\hline $\mathbf{A 0}$ & -0.00104 & -0.0055 & -0.0111 & -0.00713 & -0.03581 & -0.0047 & 0.00553 & 0.04822 & 0.01021 \\
\hline $\mathbf{A 1}$ & 0.00172 & 0.00597 & 0.01421 & 0.02632 & 0.08649 & 0.11592 & 0.07371 & 0.0066 & 0.14173 \\
\hline $\mathbf{A 2}$ & 0.00117 & 0.0039 & 0.00069 & -0.00232 & 0.00999 & -0.00064 & 0.05991 & 0.07048 & 0.06409 \\
\hline $\mathbf{A 3}$ & $-8.00 \mathrm{E}-05$ & $-9.00 \mathrm{E}-05$ & 0.00021 & 0.00039 & 0.00017 & 0.00035 & -0.00114 & -0.00035 & -0.00192 \\
\hline
\end{tabular}

The frictional resistance of the keel is given by:

$$
X_{F_{k}}=1 / 2 \rho u^{2} S_{k} C_{F_{k}}
$$

The viscous resistance of the keel is given by:

$$
X_{V_{k}}=X_{F_{k}}\left(1+2 \frac{t_{k}}{c_{k}}+60\left(\frac{t_{k}}{c_{k}}\right)^{4}\right)
$$

The frictional resistance of the rudder is given by: 


$$
\begin{gathered}
X_{F_{r}}=1 / 2 \rho u^{2} S_{r} C_{F_{r}} \\
X_{V_{r}}=X_{F_{r}}\left(1+2 \frac{t_{r}}{c_{r}}+60\left(\frac{t_{r}}{c_{r}}\right)^{4}\right)
\end{gathered}
$$

The additional residuary resistance of the canoe body in the presence of heel is given by Eq. (B.9) and Table 18 :

$$
X_{R \phi_{c}}=6 \cdot \phi^{1.7}\left(u_{0}+u_{1} \frac{L_{W L}}{B_{W L}}+u_{2}\left(\frac{B_{W L}}{D_{c}}\right)^{2}+u_{4} L_{C B}+u_{5} L_{C B}^{2}\right) \nabla_{c} \rho g-X_{F_{c}}
$$

Table 18: DSYHS Coefficients for Added Residuary Resistance due to Heel

\begin{tabular}{|c|c|c|c|c|c|c|c|}
\hline \multicolumn{7}{|c|}{ Coefficients are multiplied by 1000} \\
\hline Fn & $\mathbf{0 . 2 5}$ & $\mathbf{0 . 3 0}$ & $\mathbf{0 . 3 5}$ & $\mathbf{0 . 4 0}$ & $\mathbf{0 . 4 5}$ & $\mathbf{0 . 5 0}$ & $\mathbf{0 . 5 5}$ \\
\hline $\mathbf{u 0}$ & -0.0268 & 0.6628 & 1.6433 & -0.8659 & -3.2715 & -0.1976 & 1.5873 \\
\hline $\mathbf{u 1}$ & -0.0014 & -0.0632 & -0.2144 & -0.0354 & 0.1372 & -0.148 & -0.3749 \\
\hline $\mathbf{u 2}$ & -0.0057 & -0.0699 & -0.164 & 0.226 & 0.5547 & -0.6593 & -0.7105 \\
\hline $\mathbf{u 3}$ & 0.0016 & 0.0069 & 0.0199 & 0.0188 & 0.0268 & 0.1862 & 0.2146 \\
\hline $\mathbf{u 4}$ & -0.007 & 0.0459 & -0.054 & -0.58 & -1.0064 & -0.7489 & -0.4818 \\
\hline $\mathbf{u 5}$ & -0.0017 & -0.004 & -0.0268 & -0.1133 & -0.2026 & -0.1648 & -0.1174 \\
\hline
\end{tabular}

As the yacht heels, the underwater geometry also changes. The expression for change in wetted surface area of the hull is given by Eq. (B.10) and Table 17 :

$$
S_{c_{\phi}}=S_{c}\left(1+0.01\left(s_{0}+s_{1} \frac{B_{W L}}{D_{c}}+s_{2}\left(\frac{B_{W L}}{D_{c}}\right)^{2}+s_{3} C_{M}\right)\right)
$$

The added frictional resistance due to the change in wetted surface area is given by Eq. (B.11):

Table 17: DSYHS Coefficients for Change in Wetted Surface Area with Heel

\begin{tabular}{|c|c|c|c|c|c|c|c|}
\hline $\boldsymbol{\phi}$ (deg) & $\mathbf{5}$ & $\mathbf{1 0}$ & $\mathbf{1 5}$ & $\mathbf{2 0}$ & $\mathbf{2 5}$ & $\mathbf{3 0}$ & $\mathbf{3 5}$ \\
\hline $\mathbf{s 0}$ & -4.122 & -4.522 & -3.291 & 1.85 & 6.51 & 12.334 & 14.648 \\
\hline $\mathbf{s 1}$ & 0.054 & -0.132 & -0.389 & -1.2 & -2.305 & -3.911 & -5.182 \\
\hline $\mathbf{s 2}$ & -0.027 & -0.077 & -0.118 & -0.109 & -0.066 & 0.024 & 0.102 \\
\hline $\mathbf{s 3}$ & 6.329 & 8.738 & 8.949 & 5.364 & 3.433 & 1.767 & 3.497 \\
\hline
\end{tabular}


Table 19: DSYHS Coefficients for Added Residuary Resistance due to Heel of the Keel

\begin{tabular}{|c|c|c|c|}
\hline H1 & H2 & H3 & H4 \\
\hline-3.5837 & -0.0518 & 0.5958 & 0.2055 \\
\hline
\end{tabular}

$$
X_{F \phi_{c}}=1 / 2 \rho u^{2} C_{f} S_{c_{\phi}}
$$

The added residuary resistance of the keel due to heel is given by Eq. (B.12) and Table 19:

$$
\begin{gathered}
X_{R \phi_{k}}=\left(H_{1} \frac{D_{c}}{D_{c}+b_{k}}+H_{2} \frac{B_{W L}}{D_{c}}+H_{3} \frac{D_{c} B_{W L}}{D_{c}\left(D_{c}+b_{k}\right)}+H_{4} \frac{L_{W L}}{\nabla_{c}^{1 / 3}}\right) F n^{2} \phi \cdot \nabla_{k} \rho g \\
F n=u / \sqrt{g L_{W L}}
\end{gathered}
$$

In the presence of side velocity, meaning the yacht is sailing with leeway, there are lift and induced drag forces generated by the hull and appendages. The expression for the $\mathrm{X}$ force due to side velocity is given by Eq. (B.14) and Table 20:

$$
\begin{aligned}
X_{v}= & 1 / 2 \rho V_{B}^{2} S_{c}\left(b_{1} \frac{2}{S_{c}}+b_{2}\left(\frac{\left(D_{c}+b_{k}\right)^{2}}{S_{c}}\right)^{2}+b_{3} \frac{D_{c}}{\left(D_{c}+b_{k}\right)}+b_{4} \frac{D_{c}\left(D_{c}+b_{k}\right)^{2}}{S_{c}\left(D_{c}+b_{k}\right)}\right) \ldots \\
& \ldots\left(\frac{-v}{u}+.0092 \frac{B_{W L} D_{c}}{D_{c}\left(D_{c}+b_{k}\right)} \phi^{2} \cdot F n\right)
\end{aligned}
$$

Table 20: DSYHS Coefficients for Surge Force from Leeway

\begin{tabular}{|c|c|c|c|c|}
\hline $\boldsymbol{\phi}$ & $\mathbf{0}$ & $\mathbf{1 0}$ & $\mathbf{2 0}$ & $\mathbf{3 0}$ \\
\hline $\mathbf{b 1}$ & 2.025 & 1.989 & 1.98 & 1.762 \\
\hline $\mathbf{b 2}$ & 9.551 & 6.729 & 0.633 & -4.957 \\
\hline $\mathbf{b 3}$ & 0.631 & 0.494 & 0.194 & -0.087 \\
\hline $\mathbf{b 4}$ & -6.575 & -4.745 & -0.792 & 2.766 \\
\hline
\end{tabular}


The expression for effective span of the submerged body including the keel is used to calculate the induced drag generated by the hull/keel, and is given by Eq. (B.15) and Table 21:

$$
b_{e}=\left(A_{1} \frac{D_{c}}{\left(D_{c}+b_{k}\right)}+A_{2}\left(\frac{D_{c}}{\left(D_{c}+b_{k}\right)}\right)^{2}+A_{3} \frac{B_{W L}}{D_{c}}+A_{4} \lambda_{k}\right) \cdot\left(B_{0}+B_{1} F n\right) \cdot\left(D_{c}+b_{k}\right)
$$

Table 21: Coefficients for Induced Drag of Keel in Leeway

\begin{tabular}{|c|c|c|c|c|}
\hline $\boldsymbol{\phi}$ & $\mathbf{0}$ & $\mathbf{1 0}$ & $\mathbf{2 0}$ & $\mathbf{3 0}$ \\
\hline A1 & 3.7455 & 4.4892 & 3.9592 & 3.4891 \\
\hline $\mathbf{A 2}$ & -3.6246 & -4.8454 & -3.9804 & -2.9577 \\
\hline A3 & 0.0589 & 0.0294 & 0.0283 & 0.025 \\
\hline A4 & -0.0296 & -0.0176 & -0.0075 & -0.0272 \\
\hline & & & & \\
\hline B0 & 1.2306 & 1.4231 & 1.545 & 1.4744 \\
\hline B1 & -0.7256 & -1.2971 & -1.5622 & -1.3499 \\
\hline
\end{tabular}

The expression for the induced drag generated by the hull and keel is then given by :

$$
X_{I}=\frac{X_{v}^{2}}{\pi b_{e} 1 / 2 \rho V_{B}^{2}}
$$

The forces along the sway axis will be from the lift and induced drag of the keel/hull combination. The expression for total hydrodynamic force in the sway axis is given by:

$$
Y_{H}=Y_{v} \cos \left(\frac{-v}{u}\right)+Y_{I} \sin \left(\frac{-v}{u}\right)
$$

The expression for Y force due to side velocity is given by Eq. (B.18) and Table 20:

$$
\begin{aligned}
Y_{v}= & 1 / 2 \rho V_{B}^{2} S_{c}\left(b_{1} \frac{2}{S_{c}}+b_{2}\left(\frac{\left(D_{c}+b_{k}\right)^{2}}{S_{c}}\right)^{2}+b_{3} \frac{D_{c}}{\left(D_{c}+b_{k}\right)}+b_{4} \frac{D_{c}\left(D_{c}+b_{k}\right)^{2}}{S_{c}\left(D_{c}+b_{k}\right)}\right) \cdot \ldots \\
& \ldots\left(\frac{-v}{u}+.0092 \frac{B_{W L} D_{c}}{D_{c}\left(D_{c}+b_{k}\right)} \phi^{2} \cdot F n+\left(\frac{.43\left(D_{c}+b_{k}\right)}{u} \dot{\phi}\right)+B_{3} \phi^{2} \cdot F n\right)
\end{aligned}
$$

The expression for induced drag in the $\mathrm{Y}$ direction is given by : 


$$
Y_{I}=\frac{Y_{v}^{2}}{\pi b_{e} 1 / 2 \rho V_{B}^{2}}
$$

The moments will be calculated based on the location of the center of lateral resistance of the hull/keel combination w.r.t. the center of gravity of the yacht. The expression for hydrodynamic rolling moment is given by Eq. (B.20):

$$
K_{H}=-\Delta \overline{G M} \sin \phi+\left(Y_{v}+Y_{I}\right) \frac{.43\left(D_{c}+b_{k}\right)}{\cos \phi}=-\Delta \overline{G M} \sin \phi+Y_{H} z_{C L R}
$$

The expression for hydrodynamic yawing moment is given by Eq. (B.21)

$$
N_{H}=Y_{H} x_{C L R}
$$

Table 22: DSYHS Keel and Rudder Characteristics for the Precision 23

\begin{tabular}{|ccc|}
\hline & Keel & Rudder \\
\hline Airfoil Section & NACA 0009 & NACA 0009 \\
Aspect Ratio & 1.93 & 1.76 \\
Surface Area & $1.394 \mathrm{~m}^{2}$ & $0.232 \mathrm{~m}^{2}$ \\
$\begin{array}{c}\text { x location of } \\
\text { center of force } \\
\text { wrt c.g. } \\
\text { z location of } \\
\text { center of force } \\
\text { wrt c.g. }\end{array}$ & $1.341 \mathrm{~m}$ & $-3.35 \mathrm{~m}$ \\
\hline
\end{tabular}


Table 23: Mass Parameters for Precision 23

\begin{tabular}{|cc|cc|}
\hline $\mathrm{m}$ & $1293 \mathrm{~kg}$ & $\mathrm{I}_{\mathrm{xx}}+\mathrm{J}_{\mathrm{xx}}$ & $6074 \mathrm{~kg}-\mathrm{m}^{2}$ \\
$\mathrm{~m}_{\mathrm{x}}$ & $44 \mathrm{~kg}$ & $\mathrm{I}_{\mathrm{zz}}+\mathrm{J}_{\mathrm{zz}}$ & $10,740 \mathrm{~kg}-\mathrm{m}^{2}$ \\
$\mathrm{~m}_{\mathrm{y}}$ & $2576 \mathrm{~kg}$ & $\overline{\mathrm{GM}}$ & $1.524 \mathrm{~m}$ \\
\hline
\end{tabular}

Table 24: DSYHS Hull Parameters for Precision 23

\begin{tabular}{|c|c|c|c|c|c|}
\hline & Description & Value & & Description & Value \\
\hline $\mathrm{S}_{\mathrm{W}}$ & $\begin{array}{l}\text { Wetted surface } \\
\text { area }\end{array}$ & $9.100 \mathrm{~m}^{2}$ & $A_{w}$ & $\begin{array}{l}\text { Waterplane } \\
\text { aera }\end{array}$ & $7.47 \mathrm{~m}^{2}$ \\
\hline BWL & $\begin{array}{c}\text { Max beam @ } \\
\text { waterline }\end{array}$ & $2.042 \mathrm{~m}$ & $\mathrm{~S}_{\mathrm{Wk}}$ & $\begin{array}{c}\text { Wetted } \\
\text { surface of keel }\end{array}$ & $2.788 \mathrm{~m}^{2}$ \\
\hline LWL & $\begin{array}{l}\text { Length @ } \\
\text { waterline }\end{array}$ & $6.096 \mathrm{~m}$ & $\mathrm{t} / \mathrm{c}_{\mathrm{k}}$ & $\begin{array}{l}\text { Thickness to } \\
\text { chord of keel }\end{array}$ & 0.10 \\
\hline$t_{c}$ & $\begin{array}{l}\text { Draft of canoe } \\
\text { body }\end{array}$ & $0.180 \mathrm{~m}$ & $\nabla_{\mathrm{k}}$ & $\begin{array}{l}\text { Displacement } \\
\text { of keel }\end{array}$ & $0.044 \mathrm{~m}^{3}$ \\
\hline$\nabla$ & $\begin{array}{c}\text { Total } \\
\text { displacement }\end{array}$ & $3.240 \mathrm{~m}^{3}$ & $\mathrm{Z}_{\mathrm{k}}$ & $\begin{array}{l}\text { Vertical } \\
\text { position of } \\
\text { center } \\
\text { buoyancy, } \\
\text { keel }\end{array}$ & $0.125 \mathrm{~m}$ \\
\hline$\nabla_{\mathrm{c}}$ & $\begin{array}{l}\text { Displacement of } \\
\text { canoe body }\end{array}$ & $2.965 \mathrm{~m}^{3}$ & $b_{k}$ & Span of keel & $1.219 \mathrm{~m}$ \\
\hline lcf & $\begin{array}{l}\text { Longitudinal } \\
\text { center of } \\
\text { flotation }\end{array}$ & $-3.335 \mathrm{~m}$ & $\lambda_{\mathrm{k}}$ & $\begin{array}{l}\text { Taper ratio of } \\
\text { keel }\end{array}$ & 0.583 \\
\hline lcb & $\begin{array}{l}\text { Longitudinal } \\
\text { center of } \\
\text { buoyancy }\end{array}$ & $-3.231 \mathrm{~m}$ & $\mathrm{~S}_{\mathrm{Wr}}$ & $\begin{array}{l}\text { Wetted } \\
\text { surface of } \\
\text { rudder }\end{array}$ & $0.464 \mathrm{~m}^{2}$ \\
\hline $\mathrm{C}_{\mathrm{P}}$ & $\begin{array}{l}\text { Prismatic } \\
\text { coefficient }\end{array}$ & 0.475 & $\mathrm{t} / \mathrm{c}_{\mathrm{r}}$ & $\begin{array}{l}\text { Thickness to } \\
\text { chord of } \\
\text { rudder }\end{array}$ & 0.07 \\
\hline $\mathrm{C}_{\mathrm{M}}$ & $\begin{array}{l}\text { Midship section } \\
\text { coefficient }\end{array}$ & 0.71 & & & \\
\hline
\end{tabular}




\section{Appendix C: Linear Model Estimated from 6 ID Maneuvers}

The OSLS technique has been applied to estimate four different linear models, which will be referred to as Models 1-4 from this point on. At this point it is not possible to combine data collected on a starboard tack with that taken on a port tack because the boats behavior is dependent upon whether maneuvers are made upwind or downwind, so the same rudder or sail input on a port tack will give a different response on a starboard tack. It may be possible to resolve this issue by changing to a coordinate system in which all variables are measured relative to being upwind or downwind rather than fixed positions on the boat itself, but this has not been done at this point in time. Thus, all estimated models represent the boat sailing on a port or starboard tack, but never both. Model 1 is estimated from data collected on a starboard tack, and the entire set of estimation data which is composed of six rudder maneuvers is shown in Figure 83. As will be shown later, the fit to the Model 1 data is the worst of this set, and the ID data itself may be at fault. Of particular interest are the last two maneuvers, both rudder singlets with almost identical inputs, however, the responses in each of the states are not totally consistent between the two maneuvers. This could be due to two reasons, the first being the smoothed data has lost some of the original signal content as previously discussed, the second being the maneuver itself wasn't repeatable. It is far more likely to be the second case, and is a problem it is very possible to address.
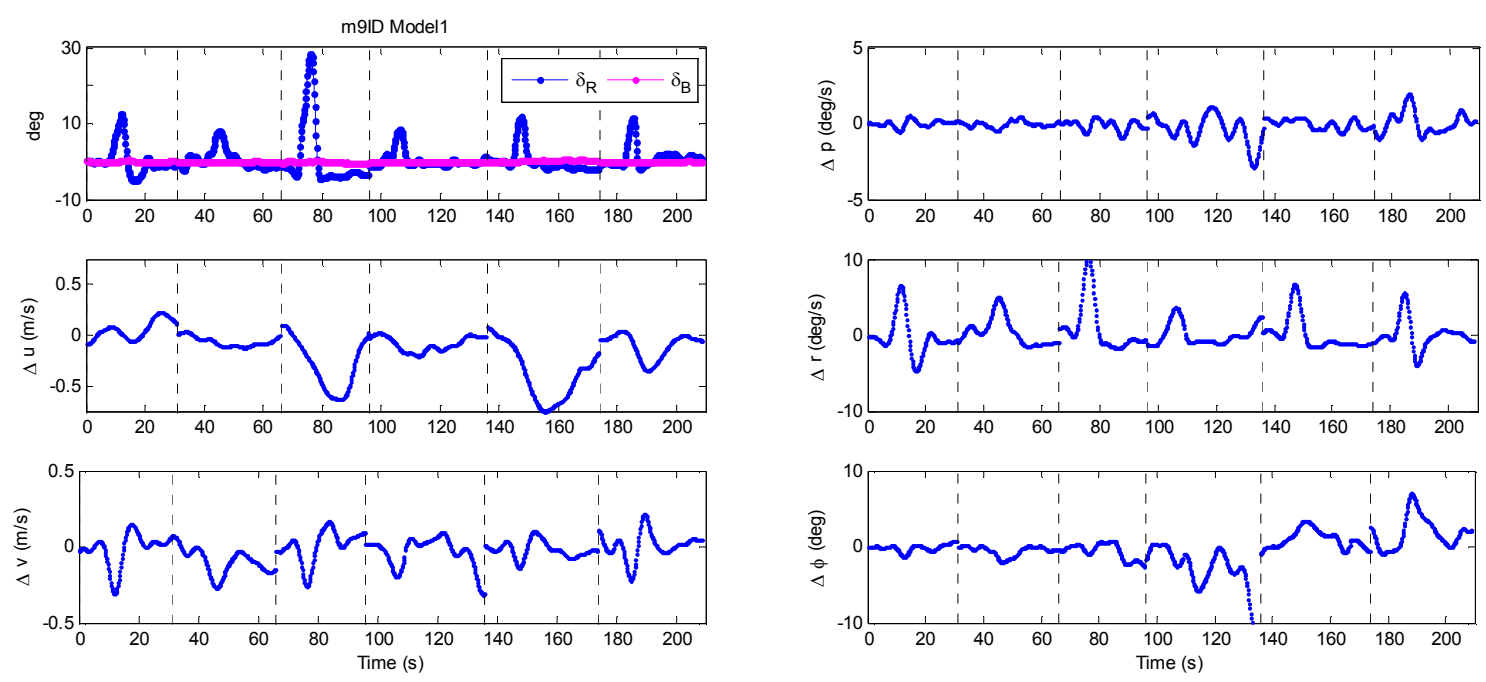

Figure 83: Model 1 ID Data 
Models 2-4 all represent very good model fits as will be discussed. The ID data for Model 4 is shown in Figure 84. Model 2 is estimated using the same data as Model 4 but without the last two maneuver segments, and Model 3 is also estimated using the same data as Model 4, but without the very last maneuver segment.

The methods presented in Section 6.4 are used to evaluate the fit to both the ID data and the TEST data. The model response to the measured inputs of each of the maneuver segments is calculated for the first five seconds of the maneuver; any longer simulation would contain too much cumulative error gathered because of the assumption that the linear model may be used to represent the real physical system. The results of this simulation for the ID data are shown in Figure 85, and for the separate TEST data in Figure 86. In these graphs, the blue represents the actual measured data, and the orange dashed line represents the simulation of the estimated model.
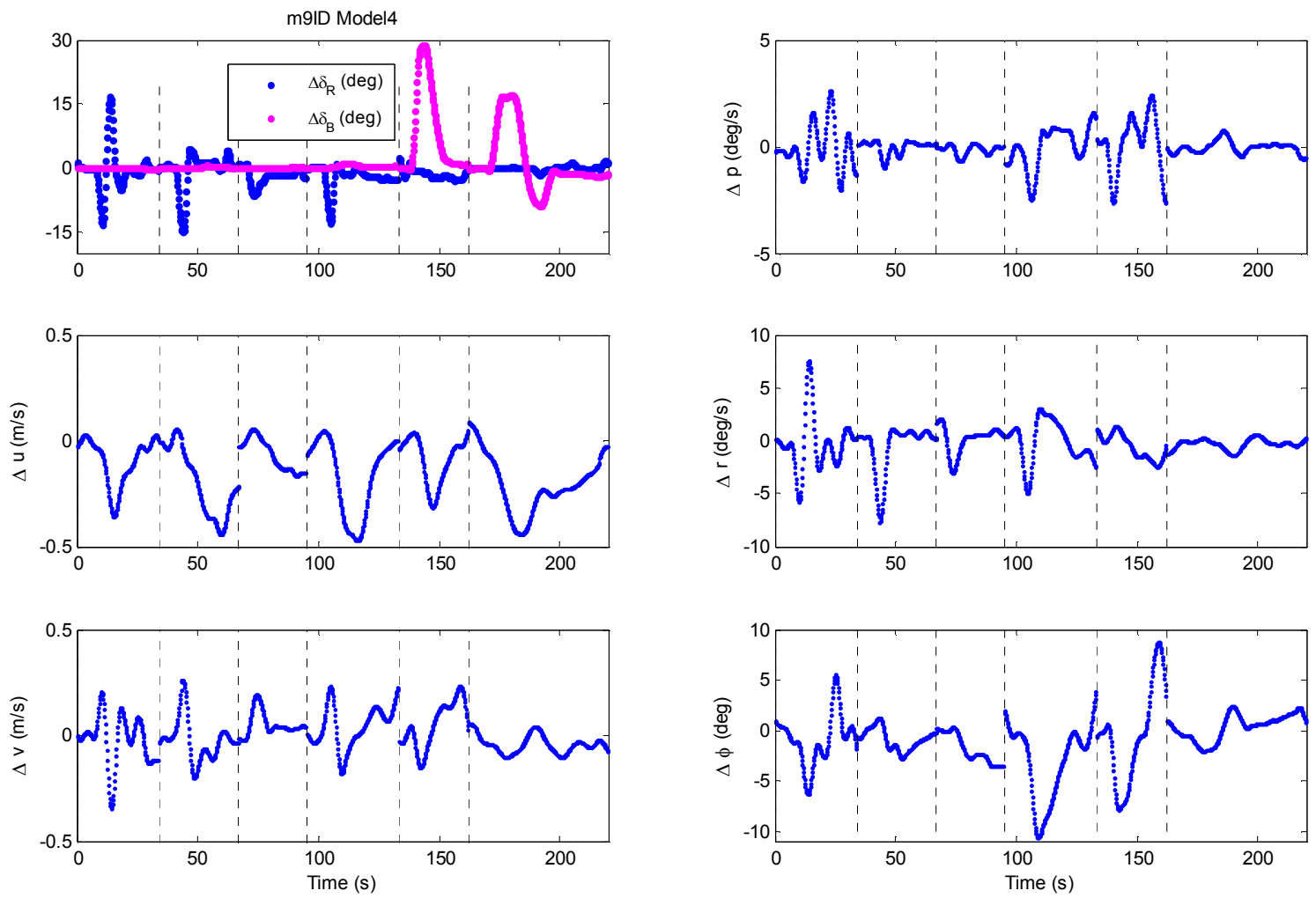

Figure 84: ID Data for Model 4 

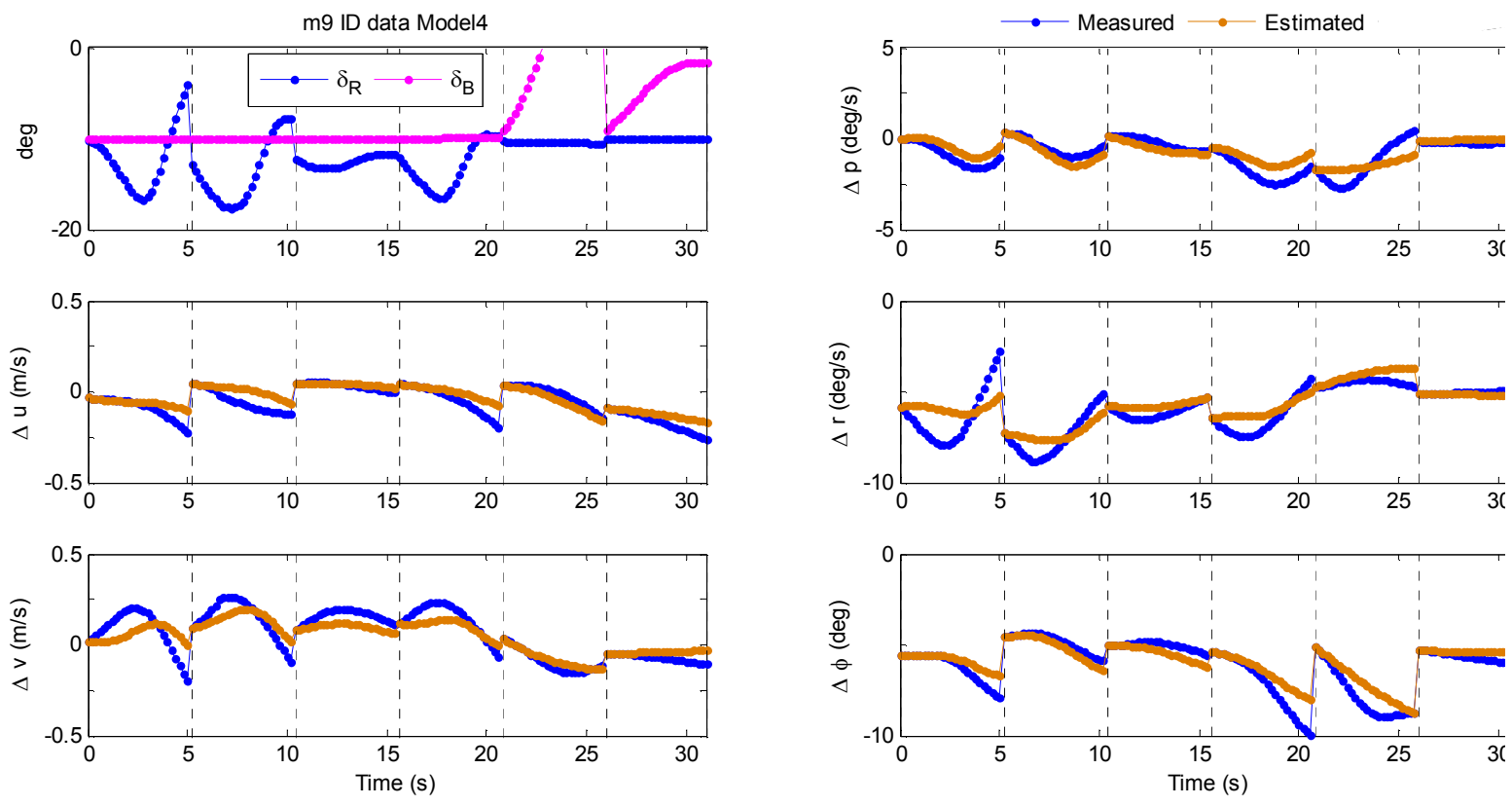

Figure 85: Fit to ID Data for Model 4
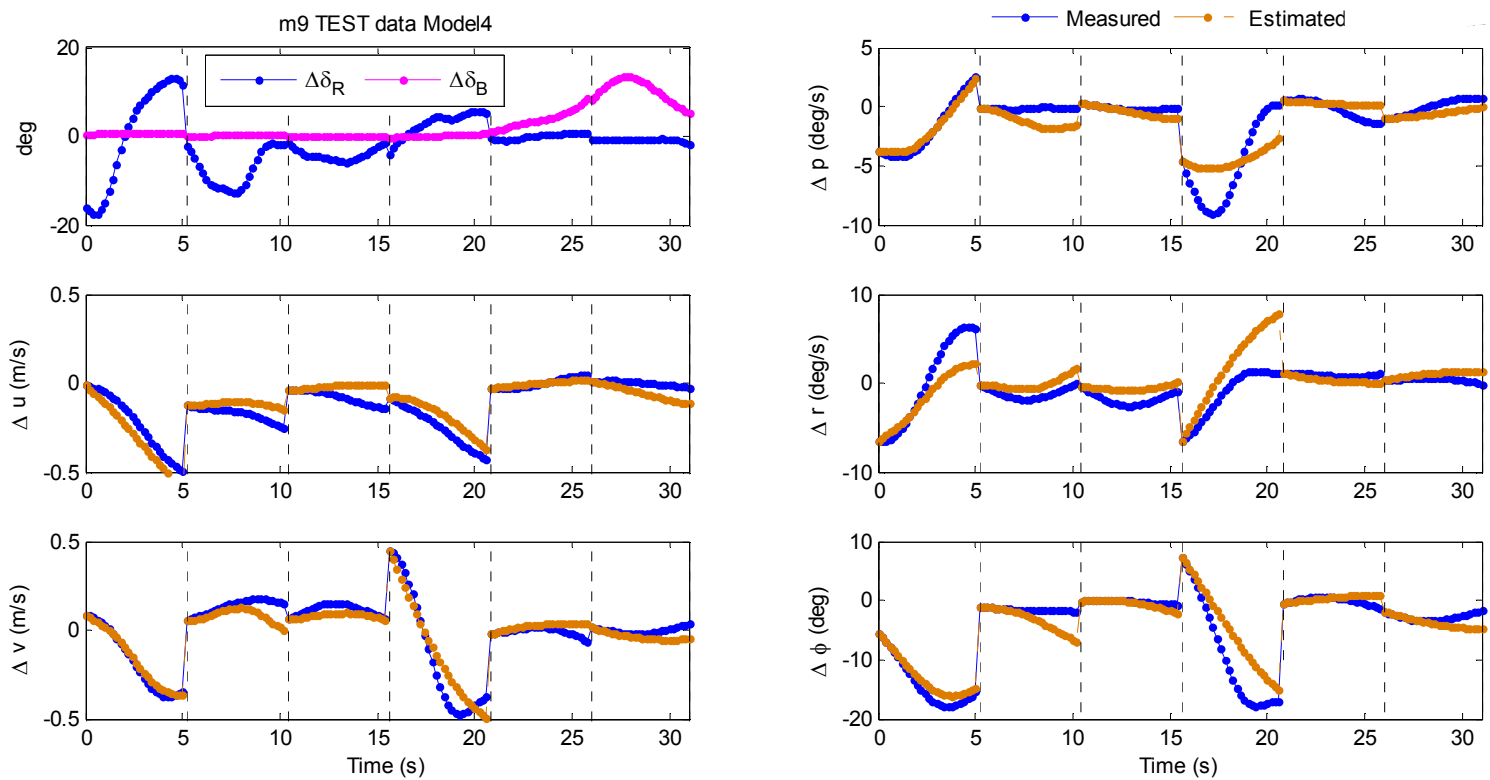

Figure 86: Fit to TEST data for Model 4 
The overall statistical evaluation of these model fits is given in Figure 87. The blue and orange in the upper left plot simply shows the number of maneuvers contained in both the ID and TEST data sets for each of the four estimated models. Below that, the green bars represent the total Theil inequality coefficient for the ID and TEST data. The threshold on this plot is set at 0.3, thus, the bars going up for Model 1 indicate a poor model fit. The bars going down for Models 2-4 are indications of a good model fit. It is interesting to note that according to $U_{\text {TOT }}$, in all three cases Models 2-4, the estimated model actually fits the TEST data better than the ID data, which normally isn't the case. The lower right graph in Figure 87 shows the R2 values calculated for the model fit. In this plot, the threshold is set at 0.75 , the bars of Models 2-4 all going up then indicate a good model fit, while the bars of Model 1 going down indicate a poor model fit. Unlike $U_{T O T}$, the $R^{2}$ in the case of Model 2 indicates that the model fits the ID data better than the TEST data, which is expected. In both Model 3 and Model $4, R^{2}$, like $U_{\text {TOT }}$ indicates that the model fits the TEST data better than the ID data. The upper right graph in Figure 87 represents this difference between the bars of the $U_{T O T}$ plot as well as the difference between the bars in the $R^{2}$ plot.
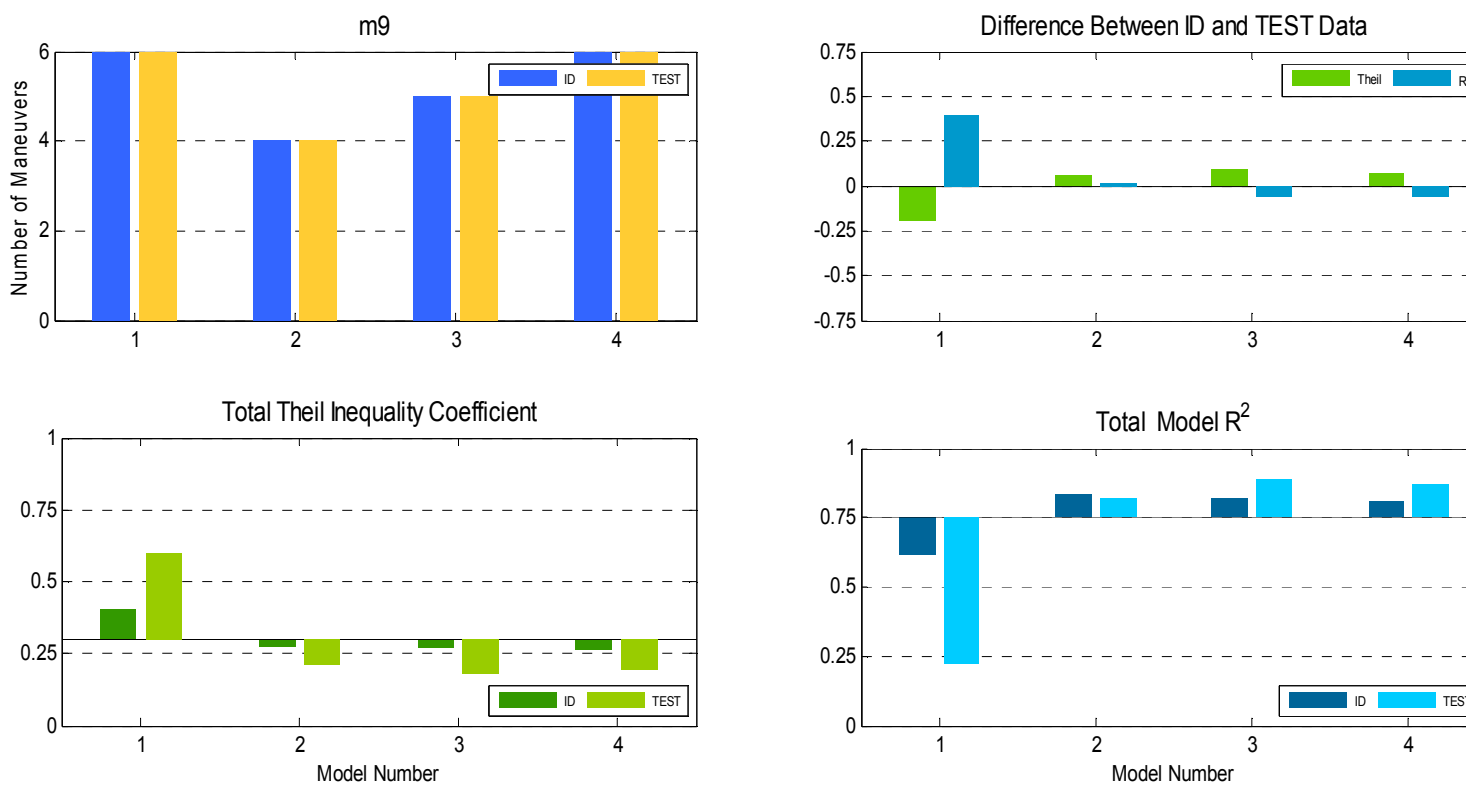

Figure 87: Statistical Result of Estimation of Models 1 - 4 

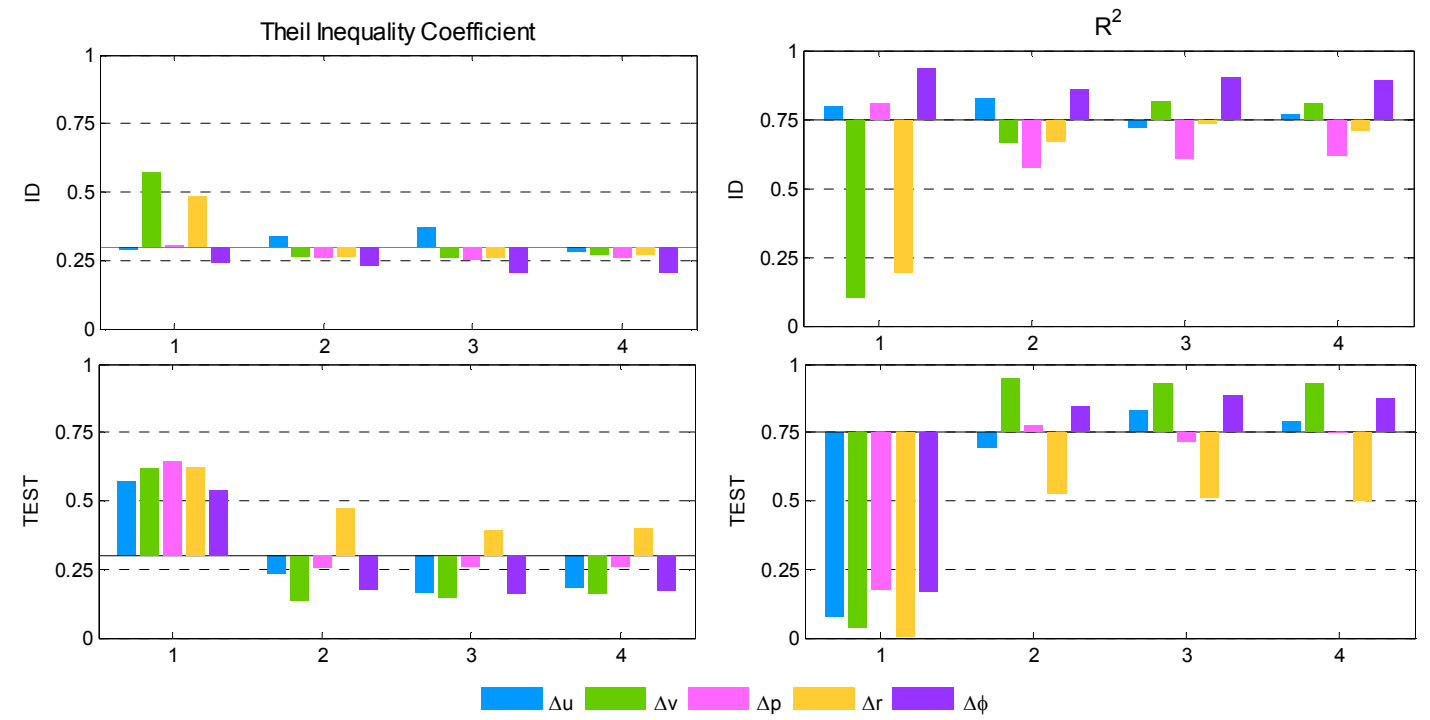

Figure 88: Breakdown of Model fit statistics into states for Models 1-4

The breakdown of the fit by both Theil inequality coefficient and $R^{2}$ is shown in Figure 88 . This lends much greater insight into which states the model is having difficulty matching. Remember that Model 1 was the worst model fit, and according to both $U$ and $R^{2}$, all states are well matched except for $\Delta v$ and $\Delta r$ in the ID data. However, all states are relatively equally poorly matched in the TEST data, so overall this was a poor model in all the states. Recall that Model 1 was also the only model estimated from starboard data, it is possible that the data collected on the starboard tack is more inconsistent than that collected on port tack. The configuration of the yacht under sail and the physical position of the sensors may be the reason for this inconsistency. So far, this kind of discrepancy has not appeared in the port data. Models 2-4 are the better fitting models. According to the Theil inequality coefficient, Models 2-4 are doing a pretty consistently good job over all the states in the ID data. However, in the TEST data, it is clear that $\Delta r$ is the only state not well matched, both according to the Theil inequality coefficient and $R^{2}$.

The last part of the statistical model analysis is the breakdown of the Theil inequality coefficient into the bias, variance, and covariance proportions. Figure 89 shows the proportions in each state calculated for the ID data fit, and Figure 90 shows the proportions in each state calculated for the TEST data fit. The bias and variance proportions, denoted by the purple and blue bars, are supposed to be less than 0.1 , thus should be going down, and the remaining 
covariance proportion in orange should be tall and going up. Since Figure 88 indicated that the poorest matching is in $\Delta r$, so that is the only state we will look at. In both the ID and TEST data, it appears as though the bias proportion is the larger contributor to error in $\Delta r$, which may be the result of the smoothing procedure not actually capturing all of the peaks in yaw.
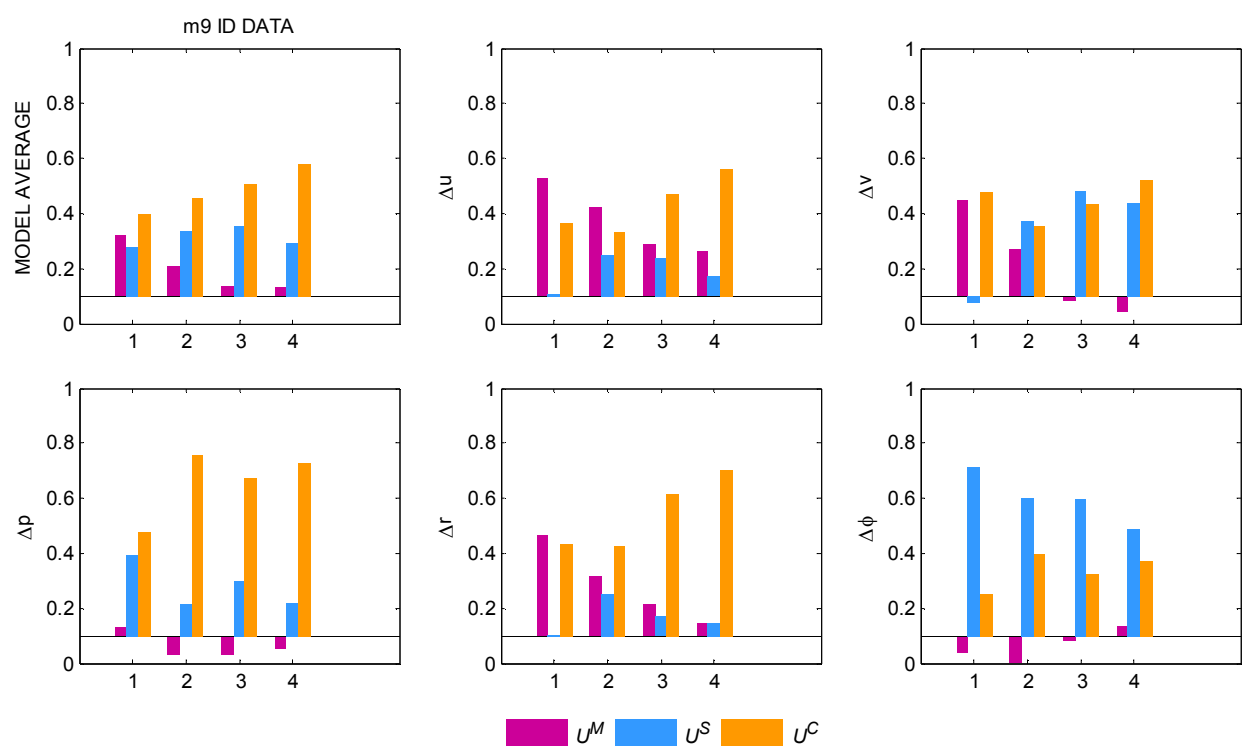

Figure 89: Breakdown of Theil inequality coefficient into bias, variance, and covariance proportions for the ID data fit
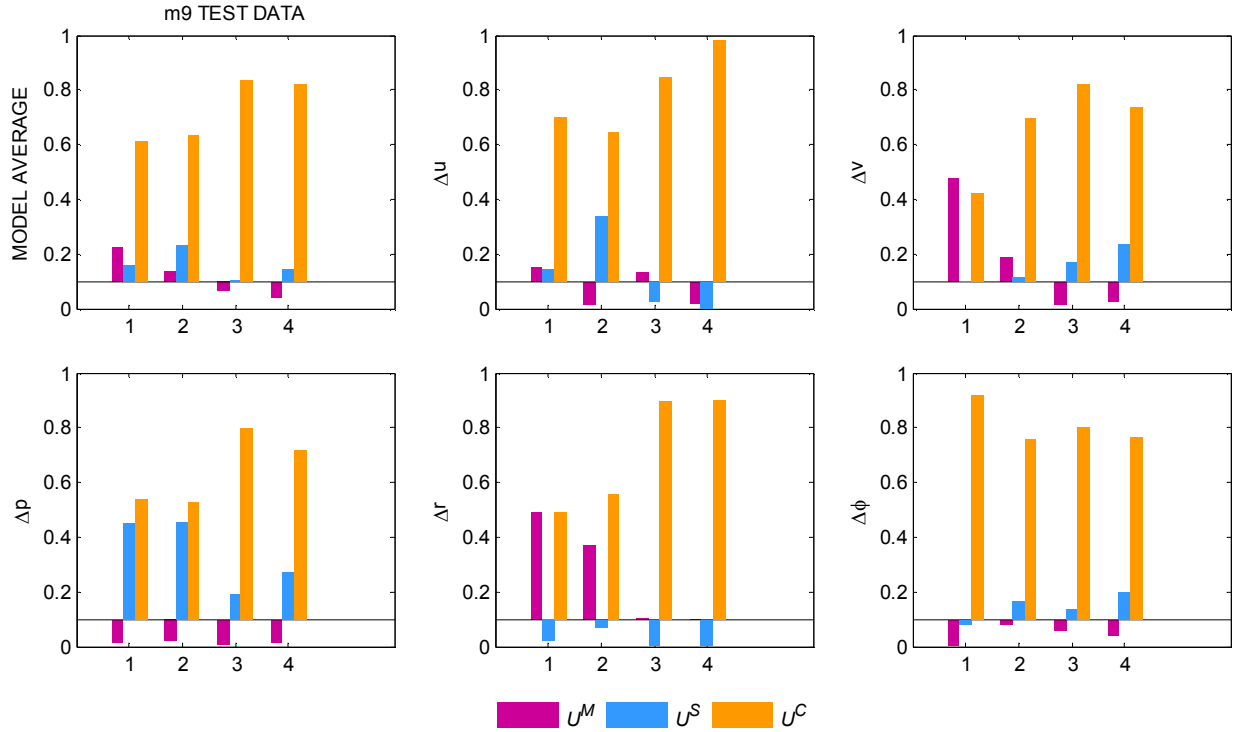

Figure 90: Breakdown of Theil inequality coefficient into bias, variance, and covariance proportions for the TEST data fit 